\title{
Exhaustive Reduction of Esters Enabled by Nickel Catalysis
}

\author{
Adam Cook, Sekar Prakash, Yan-Long Zheng, Stephen G. Newman* \\ Centre for Catalysis Research and Innovation, Department of Chemistry and Biomolecular Sciences, \\ University of Ottawa, 10 Marie Curie, Ottawa, Ontario K1N 6N5, Canada \\ *Email: stephen.newman@uottawa.ca
}

\section{Supplementary information}

\section{Table of Contents}

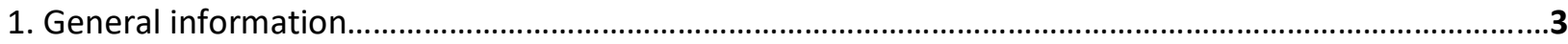

1.1. General experimental details

1.2. Instrumentation

1.3. Materials

2. Synthesis of starting materials

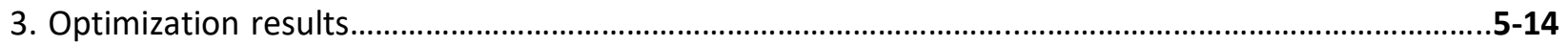

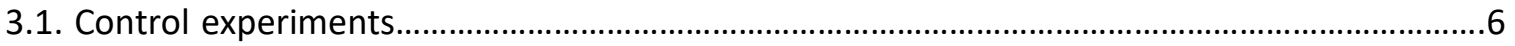

3.2. Optimization tables.............................................................................................................

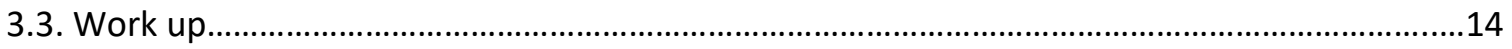

4. Reaction scope

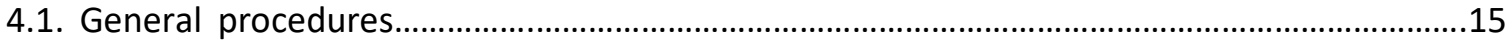

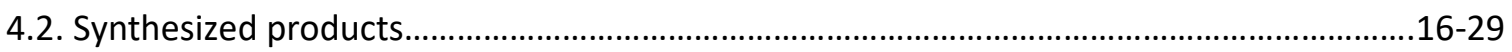


5. Troubleshooting

5.1. Scope limitations.

5.2. Improving moderate yields.

31-32

5.3. Employing a "weaker" reducing agent.

33-35

5.4. General comments. .36

6. Additional experimental information.

37-53

6.1. Catalytic deuteration .37-39

6.2. Chemoselectivity experiments 40-43

6.3. Kinetic experiments. 44-46

6.4. Reduction of proposed intermediates 47-49

6.5. Reduction of other alkyl and aryl esters 50

6.6. Kinetic-isotope experiments. 51-52

6.7. Solubility of $\mathrm{KO}^{t} \mathrm{Bu}$ 53

7. Appendix 54-57

8. NMR spectra 58-110

9. References

111-113 


\section{General considerations}

\subsection{General experimental details}

Unless otherwise indicated, reactions were conducted under an atmosphere of nitrogen in $8 \mathrm{~mL}$ screw capped vials that were oven dried $\left(120^{\circ} \mathrm{C}\right)$. Column chromatography was either performed manually using Silicycle F60 40-63 $\mu \mathrm{m}$ silica gel or by using a Combiflash Rf+ automated chromatography system with commercially available Biotage normal-phase Silica Flash columns $(35-70 \mu \mathrm{m})$. Analytical thin layer chromatography (TLC) was conducted with aluminum-backed EMD Millipore Silica Gel 60 F254 pre-coated plates. Unless otherwise noted, visualization of developed plates was performed under UV light (254 nm) and/or using $\mathrm{KMnO}_{4}$ stain.

\subsection{Instrumentation}

${ }^{1} \mathrm{H}$ NMR and ${ }^{13} \mathrm{C}$ NMR were recorded on a Bruker AVANCE $400 \mathrm{MHz}$ spectrometer. ${ }^{1} \mathrm{H}$ NMR spectra were internally referenced to the residual solvent signal (e.g., $\mathrm{CDCl}_{3}=7.27 \mathrm{ppm}$ ). ${ }^{13} \mathrm{C} \mathrm{NMR}$ spectra were internally referenced to the residual solvent signal (e.g., $\mathrm{CDCl}_{3}=77.00 \mathrm{ppm}$ ). Data for ${ }^{1} \mathrm{H} \mathrm{NMR}$ are reported as follows: chemical shift $(\delta \mathrm{ppm})$, multiplicity $(\mathrm{s}=$ singlet, $\mathrm{d}=$ doublet, $\mathrm{t}=$ triplet, $\mathrm{q}=$ quartet, quin $=$ quintet, $m=$ multiplet), coupling constant $(\mathrm{Hz})$, integration. NMR yields for optimization studies were

obtained by ${ }^{1} \mathrm{H}$ NMR analysis of the crude reaction mixture using 1,3,5-trimethoxybenzene as an internal standard. GC data was obtained via a 5-point calibration curve using FID analysis on an Agilent Technologies 7890B GC with a $30 \mathrm{~m} \times 0.25 \mathrm{~mm}$ HP-5 column. Accurate mass data (EI) was obtained from an Agilent 5977A GC/MSD using MassWorks 4.0 from CERNO Bioscience.

\subsection{Materials}

Organic solvents were purified by rigorous degassing with nitrogen before passing through a PureSolv solvent purification system. Low water content was confirmed by Karl Fischer titration $(<20 \mathrm{ppm}$ for all solvents). Unless otherwise noted, starting materials were obtained commercially from Sigma Aldrich, Alfa Aesar or Combi-Blocks and used as received. $d_{8}$-Toluene was purchased from Sigma Aldrich (99\%D). $\mathrm{Ni}(\operatorname{cod})_{2}$ was purchased from Sigma Aldrich. $\mathrm{Ni}(\mathrm{OTf})_{2}$ (96\% purity) was purchased from Alfa Aesar. $\mathrm{NiBr}_{2}$.glyme (97\% purity) was purchased from Sigma Aldrich. Granulated Mn was purchased from Alfa Aesar (99.6\% purity, < 10 micron). $D_{2}$ (g) was purchased as a $458 \mathrm{~mL}$ cylinder from Sigma Aldrich $(99.8 \%$ D). ICy. $\mathrm{HBF}_{4}$ was made according to the literature. ${ }^{1}$ 


\section{Synthesis of starting materials}

The following ester starting materials were prepared from the corresponding carboxylic acid through acid catalyzed esterification reactions: methyl [1,1'-biphenyl]-4-carboxylate (3), methyl 2-naphthoate (4), methyl phenanthrene-9-carboxylate (5), methyl 4-((1S,4R)-4-butylcyclohexyl)benzoate (6), methyl 4(dimethylamino)benzoate (7), methyl 4-vinylbenzoate (8), trans-4-(methoxycarbonyl)stilbene (9), methyl 3,4,5-trimethoxybenzoate (10), methyl 3,5-di-tert-butyl-4-hydroxybenzoate (15), methyl 4morpholinobenzoate (22), methyl 1-methyl-1H-indazole-3-carboxylate (33), methyl quinoline-6carboxylate (34) and methyl 4-(N,N-dipropylsulfamoyl)benzoate (39).

Esters including 1-methyl-1H-indole-6-carboxylate (25), 1-methyl-1H-indole-3-carboxylate (27), 1-methyl1H-indole-2-carboxylate (28), methyl 7-methoxybenzofuran-2-carboxylate (35), isochroman-1-one (37), Bifendatatum (38) and methyl (E)-3-([1,1'-biphenyl]-4-yl)acrylate (45) were purchased commercially and used as such for the reduction reaction.

The following ester starting materials were synthesized according to the noted citations: methyl 4(phenoxy)benzoate (11), ${ }^{2}$ methyl 4-(4-methoxyphenoxy)benzoate (12), ${ }^{3}$ methyl 4-(4phenoxyphenoxy)benzoate (13), ${ }^{4}$ methyl 2 '-hydroxy-[1,1'-biphenyl]-4-carboxylate (14), ${ }^{5}$ methyl 6phenylnicotinate (17), ${ }^{6}$ methyl 6-(2-methoxyphenyl)nicotinate $(45),{ }^{6}$ methyl 4 -(pyridin-2-yl)benzoate (18), ${ }^{6}$ methyl 4-(4-(pyridin-2-yl)piperazin-1-yl)benzoate (19), ${ }^{7}$ methyl 6-morpholinonicotinate (20), ${ }^{7}$ methyl 2-morpholinobenzoate (21), ${ }^{8}$ methyl 2-(4-methylpiperidin-1-yl)benzoate (23), ${ }^{8}$ methyl 4-(1,4dioxa-8-azaspiro[4.5] decan-8-yl)benzoate (24), ${ }^{8}$ methyl 4-(1H-indol-1-yl)benzoate (29), ${ }^{9}$ methyl 9-benzyl$9 H$-carbazole-3-carboxylate (30), ${ }^{10}$ methyl 6-(tert-butyl)-9-methyl-9H-carbazole-3-carboxylate $(32),{ }^{10}$ methyl 6-methoxy-9-methyl-9H-carbazole-3-carboxylate (31), ${ }^{11}$ methyl 8-methoxydibenzo[b,d]furan-2carboxylate (36) ${ }^{12}$. 4'-(methoxycarbonyl)biphenyl-4-carboxylic acid (16), methyl 4'-fluoro-1,1'-biphenyl-4carboxylate (40), methyl 4'-trifluoromethylbiphenyl-4-carboxylate (41), methyl 4'nitro-4- 
biphenylcarboxylate (42), 4-(3-pentyn-1-yloxy)-carboxylate (43), and 4-(2-furanyl)-methyl benzoate (44) were synthesized by Suzuki reactions according to literature precedent. ${ }^{5}$

\section{Reaction optimization}

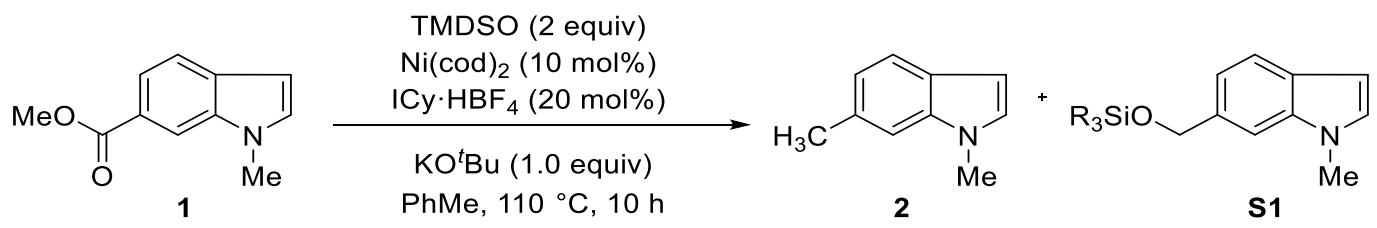

Scheme S1. General method for the reduction of esters

To an oven dried $8 \mathrm{~mL}$ screw-top test-tube, $0.20 \mathrm{mmol}$ of methyl ester starting material is added alongside an oven dried micro-stir bar. The screw-top test-tube is subsequently brought into a nitrogen-filled glovebox. Once under the inert atmosphere, $\left.0.04 \mathrm{mmol} \mathrm{ICy} \cdot \mathrm{HBF}_{4}, 0.02 \mathrm{mmol} \mathrm{Ni(cod}\right)_{2}$ and $0.20 \mathrm{mmol}$ of $\mathrm{KO}^{\mathrm{t}} \mathrm{Bu}$ are added. $0.8 \mathrm{~mL}$ of toluene is added to the test-tube, followed by $0.4 \mathrm{mmol}$ of 1,1,3,3-tetramethyldisiloxane. The reaction vessel is quickly sealed with a Teflon-septa equipped cap and brought outside of the glovebox, where it is stirred inside of a mineral-oil bath at $600 \mathrm{rpm}$ for 10 hours at $110^{\circ} \mathrm{C}$. After 10 hours, the reaction vessel is allowed to come to room temperature before being opened to the atmosphere. $0.80 \mathrm{mmol}$ of tetra-n-butylammonium fluoride is added slowly as a 1.0 $\mathrm{M}$ solution in tetrahydrofuran and the resulting solution is heated to $65^{\circ} \mathrm{C}$ and stirred for 2 hours. ${ }^{*}$ The reaction solution is filtered through a short plug of silica before $100 \mu \mathrm{L}$ of $1,3,5$ trimethoxybenzene in toluene is added to act as an internal standard. Solvent is removed via rotary evaporation, yielding crude product which is subsequently dissolved in $0.75 \mathrm{~mL}$ of $\mathrm{CDCl}_{3}$ before being submitted for NMR analysis. All yields are obtained via ${ }^{1} \mathrm{H}-\mathrm{NMR}$, setting the integral value of the peak corresponding to the three methyl protons at $2.54 \mathrm{ppm}$ to 3.00 and referencing it with respect to the peak at 6.08 ppm for 1,3,5-trimethoxybenzene.

${ }^{*} \mathbf{S} 1$ is initially formed as a mixture of silylated species - TBAF is added to the reaction solution in order to deprotect these species so that the alcohol can be quantified. 


\subsection{Control Experiments}

Table S1. Control experiments for the ester to methyl reduction
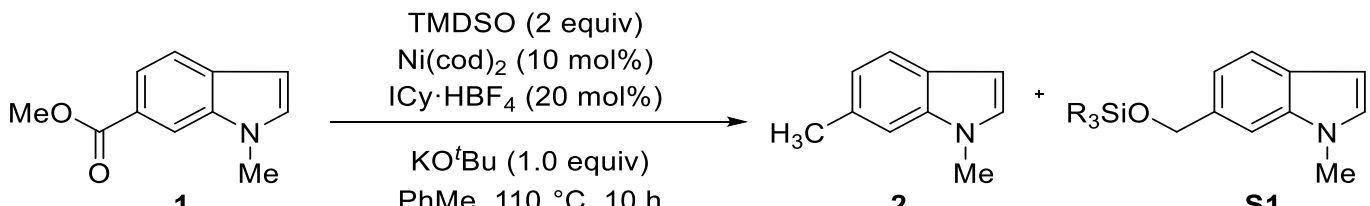

\begin{tabular}{cccc}
\hline entry & deviation from standard conditions & yield 2 [\%] & yield S1 [\%] \\
\hline $\mathbf{1}$ & No deviation & 84 & 0 \\
$\mathbf{2}$ & remove $\mathrm{KO}^{\mathrm{B}} \mathrm{Bu}$ & 0 & 0 \\
$\mathbf{3}$ & remove $\mathrm{TMDSO}$ & 0 & 0 \\
$\mathbf{4}$ & remove $\mathrm{Ni}(\mathrm{cod})_{2}$ & trace & 52 \\
$\mathbf{5}$ & remove $\mathrm{ICy} \cdot \mathrm{HBF}$ & & 0 \\
$\mathbf{6}$ & remove all except $\mathrm{TMDSO}$ and KOํ $\mathrm{Bu}$ & 14 & 66 \\
$\mathbf{7}$ & remove all except $\mathrm{Ni}(\mathrm{cod})_{2}$ & trace & 0 \\
$\mathbf{8}$ & room temperature & 0 & 61 \\
$\mathbf{9}$ & $1 \mathrm{~h}$ & 14 & 67 \\
\hline
\end{tabular}




\subsection{Optimization of reaction conditions}

Table S2. Organosilane optimization for the ester to methyl reduction

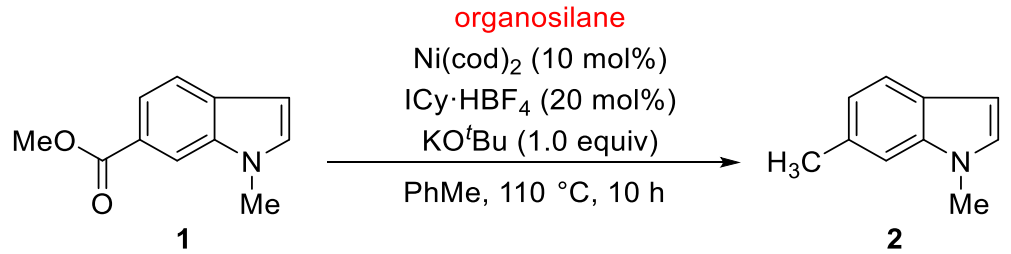

\begin{tabular}{|c|c|c|}
\hline entry & organosilane & yield 2 [\%] \\
\hline 1 & $\mathrm{Et}_{3} \mathrm{SiH}$ (4.0 equiv) & 42 \\
\hline 2 & ${ }^{i} \mathrm{Pr}_{3} \mathrm{SiH}$ (4.0 equiv) & 5 \\
\hline 3 & $(\mathrm{EtO})_{3} \mathrm{SiH}$ (4.0 equiv) & 20 \\
\hline 4 & PMHS (10.0 equiv) & trace \\
\hline 5 & $\mathrm{PhSiH}_{3}$ (2.0 equiv) & trace \\
\hline 6 & {$\left[\left(\mathrm{Me}_{3} \mathrm{SiO}\right)_{2} \mathrm{SiHMe}\right]$ (4.0 equiv) } & 48 \\
\hline 7 & $\left.\left[\left(\mathrm{CH}_{3}\right)_{2} \mathrm{SiHO}\right)_{2} \mathrm{Si}\left(\mathrm{CH}_{3}\right)_{2}\right]$ (4.0 equiv) & 65 \\
\hline 8 & TMDSO ( 1.0 equiv) & 52 \\
\hline 9 & TMDSO (1.5 equiv) & 76 \\
\hline 10 & TMDSO (2.0 equiv) & 84 \\
\hline 11 & TMDSO ( 2.5 equiv) & 74 \\
\hline
\end{tabular}


Table S3. Base optimization for the ester to methyl reduction

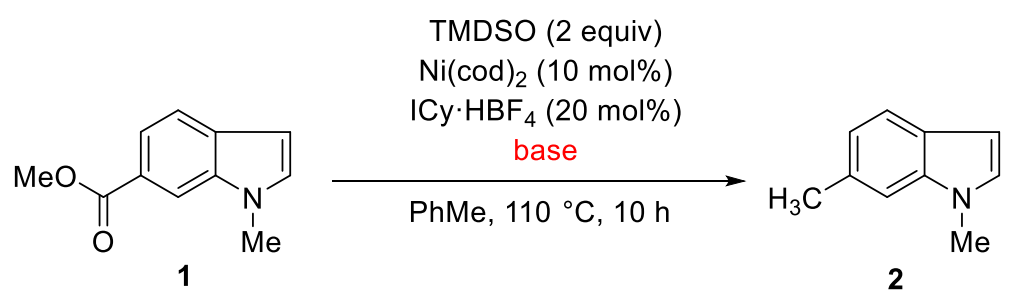

\begin{tabular}{|c|c|c|}
\hline entry & base & yield, 2 [\%] \\
\hline 1 & $\mathrm{NaOH}(1.0 \mathrm{eq})$ & 4 \\
\hline 2 & $\mathrm{LiOH}$ (1.0 eq) & 0 \\
\hline 3 & $\mathrm{KOH}(1.0 \mathrm{eq})$ & 12 \\
\hline 4 & $\mathrm{Et}_{3} \mathrm{~N}(1.0 \mathrm{eq})$ & 0 \\
\hline 5 & $\mathrm{Cs}_{2} \mathrm{CO}_{3}(1.0 \mathrm{eq})$ & 0 \\
\hline 6 & $\mathrm{~K}_{2} \mathrm{CO}_{3}(1.0 \mathrm{eq})$ & 3 \\
\hline 7 & $\mathrm{NMe}_{4} \mathrm{OH} \cdot 5 \mathrm{H}_{2} \mathrm{O}(1.0 \mathrm{eq})$ & 37 \\
\hline 8 & $\mathrm{KHF}_{2}(1.0$ eq $)$ & 0 \\
\hline 9 & $\mathrm{NaOMe}(1.0 \mathrm{eq})$ & 56 \\
\hline 10 & KOEt (1.0 eq) & 51 \\
\hline 11 & $\mathrm{LiO}^{t} \mathrm{Bu}(1.0 \mathrm{eq})$ & trace \\
\hline 12 & $\mathrm{NaO}^{t} \mathrm{Bu}(1.0 \mathrm{eq})$ & 75 \\
\hline 13 & $\mathrm{KO}^{t} \mathrm{Bu}(1 \mathrm{eq})$ & 78 \\
\hline 14 & $\mathrm{KO}^{t} \mathrm{Bu}(0.5 \mathrm{eq})$ & 69 \\
\hline 15 & $\mathrm{KO}^{t} \mathrm{Bu}(1.5 \mathrm{eq})$ & 74 \\
\hline 16 & $\mathrm{KO}^{t} \mathrm{Bu}(2.0 \mathrm{eq})$ & 68 \\
\hline
\end{tabular}


Table S4. Optimization of metal source for the ester to methyl reduction

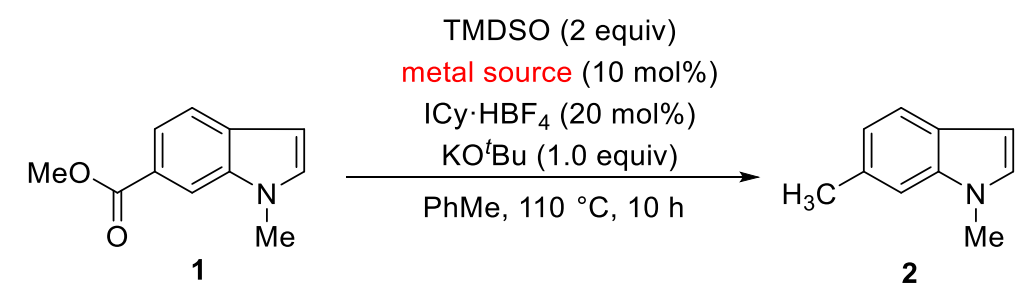

\begin{tabular}{|c|c|c|}
\hline entry & Metal source & yield, 2 [\%] \\
\hline 1 & $\mathrm{Ni}(\mathrm{cod})_{2}$ & 84 \\
\hline 2 & $\mathrm{NiCl}_{2}$ & 4 \\
\hline 3 & $\mathrm{Ni}\left(\mathrm{P}(\mathrm{OPh})_{3}\right)_{4}$ & 0 \\
\hline 4 & $\mathrm{NiCl}_{2}\left(\mathrm{PC}_{3}\right)_{2}$ & 67 \\
\hline 5 & $\mathrm{Ni}(\mathrm{OAc})_{2}$ & trace \\
\hline 6 & $\mathrm{Ni}(\mathrm{OTf})_{2}$ & 5 \\
\hline 7 & $\mathrm{NiBr}_{2} \cdot$ glyme & 7 \\
\hline 8 & $\mathrm{PdCl}_{2}$ & 29 \\
\hline 9 & $\mathrm{Pd}(\mathrm{OAc})_{2}$ & 28 \\
\hline 10 & $\mathrm{Pd}_{2} \mathrm{dba}_{3}$ & 34 \\
\hline 11 & $\mathrm{RhCl}\left(\mathrm{PPh}_{3}\right)_{3}$ & trace \\
\hline 12 & $\mathrm{Pt} / \mathrm{C}$ & 11 \\
\hline 13 & $\operatorname{Ir}(\operatorname{cod}){ }_{2} \mathrm{Cl}_{2}$ & 13 \\
\hline 14 & {$\left[\mathrm{Ru}(\mathrm{p} \text {-cymene }) \mathrm{Cl}_{2}\right]_{2}$} & 7 \\
\hline 15 & Cul & 0 \\
\hline 16 & $\mathrm{FeCl}_{3}$ & 0 \\
\hline 17 & $\mathrm{Ni}(\operatorname{cod})_{2}(5 \mathrm{~mol} \%)$ & 62 \\
\hline 18 & $\mathrm{Ni}(\operatorname{cod})_{2}(20 \mathrm{~mol} \%)$ & 86 \\
\hline 19 & $\mathrm{Ni}(\operatorname{cod})_{2}(30 \mathrm{~mol} \%)$ & 82 \\
\hline
\end{tabular}


Table S5. Optimization of reaction with air stable Ni(II) salts

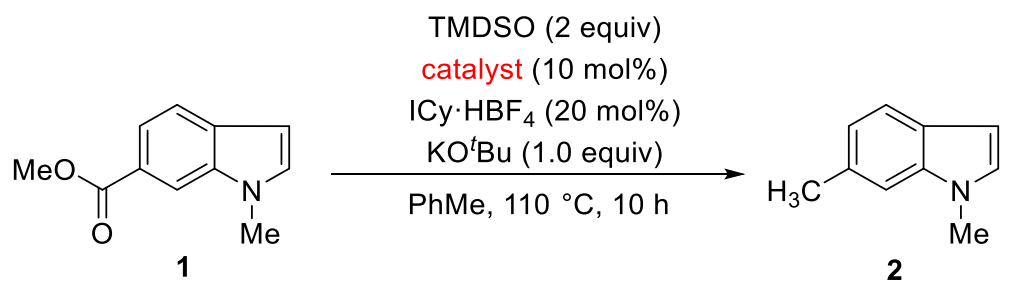

\begin{tabular}{|c|c|c|c|}
\hline entry & catalyst & deviation from standard procedure & yield, 2 [\%] \\
\hline 1 & $\mathrm{Ni}(\mathrm{OAc})_{2}$ & none & trace \\
\hline 2 & $\mathrm{Ni}(\mathrm{OAc})_{2}$ & added $\mathrm{Mn}(1 \mathrm{eq})$ & 56 \\
\hline 3 & $\mathrm{Ni}(\mathrm{OAc})_{2}$ & added Mn (20 mol\%) & 13 \\
\hline 5 & $\mathrm{Ni}(\mathrm{OAc})_{2}$ & added $\mathrm{Mn}(1 \mathrm{eq}) /$ no TMDSO & 0 \\
\hline 6 & $\mathrm{Ni}(\mathrm{OAc})_{2}$ & added $\mathrm{Mn}$ (1 eq)/no TMDSO/no base & 0 \\
\hline 7 & $\mathrm{Ni}(\mathrm{OAc})_{2}$ & added $\mathrm{Mn}(1 \mathrm{eq}) / 0.2$ eq base & 27 \\
\hline 8 & $\mathrm{Ni}(\mathrm{OAc})_{2}$ & added $\mathrm{Mn}$ (1 eq)/no base & trace \\
\hline 9 & $\mathrm{Ni}(\mathrm{OAc})_{2}$ & added $\mathrm{Mn}(1 \mathrm{eq}) /$ no ICy & 33 \\
\hline 10 & $\mathrm{NiBr}_{2} \cdot$ glyme & none & 7 \\
\hline 11 & $\mathrm{NiBr}_{2} \cdot$ glyme & added $\mathrm{Mn}(1 \mathrm{eq})$ & 78 \\
\hline 12 & $\mathrm{NiBr}_{2} \cdot$ glyme & added Mn (20 mol\%) & 35 \\
\hline
\end{tabular}


Table S6. Ligand optimization for the ester to methyl reduction

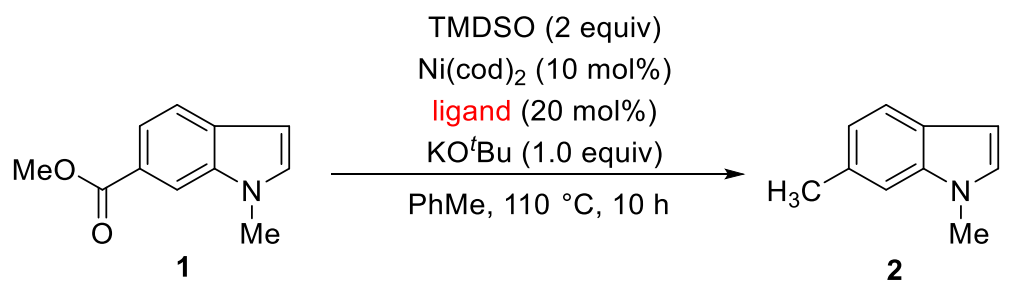

\begin{tabular}{|c|c|c|}
\hline entry & ligand & yield, 2 [\%] \\
\hline 1 & $\mathrm{PCy}_{3}$ & 53 \\
\hline 2 & $\mathrm{PPh}_{3}$ & 15 \\
\hline 3 & JohnPhos & 16 \\
\hline 4 & Xantphos & 35 \\
\hline 5 & $N$-Xantphos & 29 \\
\hline 6 & dppf & 27 \\
\hline 7 & dcype & 44 \\
\hline 8 & dppent & 35 \\
\hline 9 & IMes $\cdot \mathrm{HCl}$ & 3 \\
\hline 10 & $\mathrm{SIPr} \cdot \mathrm{HCl}$ & 53 \\
\hline 11 & $\mathrm{IPr} \cdot \mathrm{HCl}$ & 70 \\
\hline 12 & $\mathrm{ICy} \cdot \mathrm{HCl}$ & 77 \\
\hline 13 & $\mathrm{ICy} \cdot \mathrm{HBF}_{4}$ & 84 \\
\hline 14 & $\mathrm{I}^{t} \mathrm{Bu} \cdot \mathrm{HBF}_{4}$ & 73 \\
\hline 15 & ICyp $\cdot \mathrm{HBF}_{4}$ & 60 \\
\hline
\end{tabular}


<smiles>C1CCC(P(C2CCCCC2)C2CCCCC2)CC1</smiles>

$\mathrm{PCy}_{3}$<smiles>c1ccc(P(c2ccccc2)c2ccccc2)cc1</smiles>

$\mathrm{PPh}_{3}$<smiles>CC(C)(C)P(c1ccccc1-c1ccccc1)C(C)(C)C</smiles>

JohnPhos<smiles>CC1(C)c2cccc(P(c3ccccc3)c3ccccc3)c2Oc2c(P(c3ccccc3)c3ccccc3)cccc21</smiles>

Xantphos<smiles>c1ccc(P(c2ccccc2)c2cccc3c2Oc2c(cccc2P(c2ccccc2)c2ccccc2)N3)cc1</smiles>

$N$-Xantphos

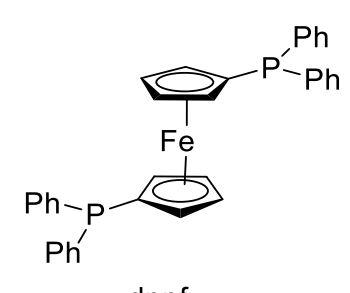

dppf<smiles></smiles><smiles>c1ccc(P(CCCP(c2ccccc2)c2ccccc2)c2ccccc2)cc1</smiles>
dppent<smiles>Cc1cc(C)c(-n2cc[n+](-c3c(C)cc(C)cc3C)c2)c(C)c1</smiles>

IMes $\cdot \mathrm{HCl}$<smiles>CC(C)c1cccc(C(C)C)c1N1C=[N+](c2c(C(C)C)cccc2C(C)C)CC1</smiles>

$\mathrm{SIPr} \cdot \mathrm{HCl}$<smiles>CC(C)c1cccc(C(C)C)c1-n1cc[n+](-c2c(C(C)C)cccc2C(C)C)c1</smiles>

$\mathrm{IPr} \cdot \mathrm{HCl}$<smiles>Cl[C-]1CCCCC1n1cc[n+](C2CCCCC2)c1</smiles>

$\mathrm{ICy} \cdot \mathrm{HCl}$<smiles></smiles>

ICy. $\mathrm{HBF}_{4}$<smiles></smiles>

$I^{t} \mathrm{Bu} \cdot \mathrm{HBF}_{4}$

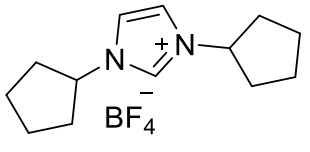

ICyp $\cdot \mathrm{HBF}_{4}$

Scheme S2. Structures of ligands 
Table S7. Miscellaneous optimization for the ester to methyl reduction
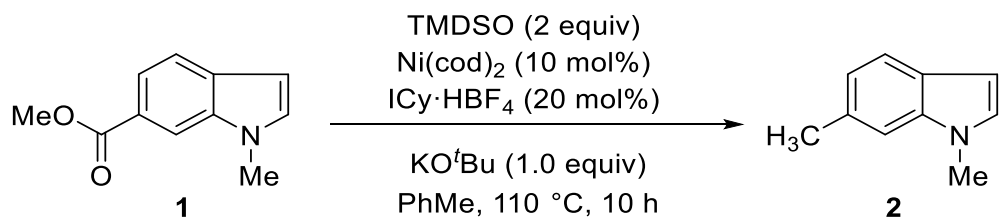

\begin{tabular}{ccc}
\hline entry & deviation from standard conditions & yield, $\mathbf{2}$ [\%] \\
\hline $\mathbf{1}$ & temperature $=80^{\circ} \mathrm{C}$ & 51 \\
$\mathbf{2}$ & temperature $=100^{\circ} \mathrm{C}$ & 71 \\
$\mathbf{3}$ & temperature $=120^{\circ} \mathrm{C}$ & 81 \\
$\mathbf{4}$ & temperature $=150^{\circ} \mathrm{C}$ & 67 \\
$\mathbf{5}$ & reaction time $=3 \mathrm{~h}$ & 25 \\
$\mathbf{6}$ & reaction time $=6 \mathrm{~h}$ & 54 \\
$\mathbf{7}$ & reaction time $=9 \mathrm{~h}$ & 79 \\
$\mathbf{8}$ & reaction time $=10 \mathrm{~h}$ & 84 \\
$\mathbf{9}$ & reaction time $=16 \mathrm{~h}$ & 81 \\
$\mathbf{1 0}$ & solvent $=\mathrm{xylene}$ & 71 \\
$\mathbf{1 1}$ & solvent $=\mathrm{DMF}$ & 0 \\
$\mathbf{1 2}$ & solvent $=$ benzene & 78 \\
\hline
\end{tabular}




\subsection{Work-up}
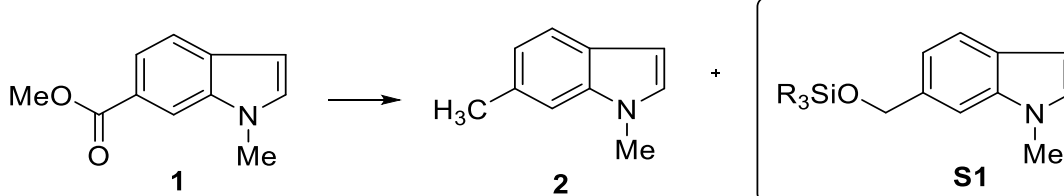

TBAF (8 eq)

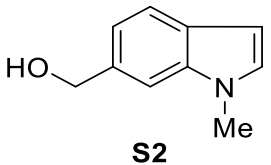

The major side products in the reduction of esters to tolyl derivatives are the corresponding silylated alcohol (e.g. S1) and various siloxane derivatives arising from the TMDSO. While the quantity of silylated alcohol is minimized by running the reaction to completion, purification can still be hindered by the siloxane byproducts. This is particularly true when purifying non-polar products by silica gel chromatography. We found that quenching the reaction with 8 equivalents of $1.0 \mathrm{M} \mathrm{TBAF}$ in THF and stirring for $2 \mathrm{~h}$ at $65^{\circ} \mathrm{C}$ (or alternatively, $6 \mathrm{~h}$ at room temperature) prior to isolation was beneficial. While not mandatory, this work-up procedure was followed throughout to ensure the products obtained were spectroscopically pure. 


\section{Reaction scope}

\subsection{General procedures}

\section{General procedure A}

To an oven dried $8 \mathrm{~mL}$ screw-top test-tube, $0.20 \mathrm{mmol}$ of methyl ester starting material is added alongside an oven dried micro-stir bar. The screw-top test-tube is subsequently brought into a nitrogen-filled glovebox. Once under the inert atmosphere, $\left.0.04 \mathrm{mmol} \mathrm{ICy} \cdot \mathrm{HBF}_{4}, 0.02 \mathrm{mmol} \mathrm{Ni(cod}\right)_{2}$ and $0.20 \mathrm{mmol}$ of $\mathrm{KO}^{\mathrm{t}} \mathrm{Bu}$ are added. $0.8 \mathrm{~mL}$ of toluene is added to the test-tube, followed by $0.40 \mathrm{mmol}$ of $1,1,3,3-$ tetramethyldisiloxane. The reaction vessel is quickly sealed with a Teflon-septa equipped cap and brought outside of the glovebox, where it is stirred inside of a mineral-oil bath at $600 \mathrm{rpm}$ for 10 hours at $110^{\circ} \mathrm{C}$. After 10 hours, the reaction vessel is allowed to come to room temperature before being opened to the atmosphere. $0.80 \mathrm{mmol}$ of tetra-n-butylammonium fluoride is added slowly as a $1.0 \mathrm{M}$ solution in tetrahydrofuran and the resulting solution is heated to $65^{\circ} \mathrm{C}$ and stirred for 2 hours. The crude reaction solution is subsequently added directly to a $10 \mathrm{~g}$ Biotage SNAP silica-packed column where it is purified on a CombiFlash Rf+ automated chromatography instrument using a mixture of ethyl acetate in hexanes.

\section{General procedure B - glovebox free conditions}

To an $8 \mathrm{~mL}$ screw-top test-tube, $0.20 \mathrm{mmol}$ of methyl ester starting material is added alongside a microstir bar. $0.20 \mathrm{mmol} \mathrm{Mn}, 0.02 \mathrm{mmol} \mathrm{NiBr} 2 \cdot$ glyme, $0.04 \mathrm{mmol} \mathrm{ICy} \cdot \mathrm{HBF}_{4}$ and $0.20 \mathrm{mmol}$ of $\mathrm{KO}^{t} \mathrm{Bu}$ are subsequently added. $0.8 \mathrm{~mL}$ of toluene is introduced to the test-tube, followed by $0.40 \mathrm{mmol}$ of $1,1,3,3$ tetramethyldisiloxane. The reaction vessel is quickly sealed with a Teflon-septa equipped cap and stirred inside of a mineral-oil bath at $600 \mathrm{rpm}$ for 10 hours at $110^{\circ} \mathrm{C}$. After 10 hours, the reaction vessel is allowed to come to room temperature before being opened to the atmosphere. $0.80 \mathrm{mmol}$ of tetra-nbutylammonium fluoride is added slowly as a $1.0 \mathrm{M}$ solution in tetrahydrofuran and the resulting solution is heated to $65^{\circ} \mathrm{C}$ and stirred for 2 hours. The crude reaction solution is subsequently added directly to a $10 \mathrm{~g}$ Biotage SNAP silica-packed column where it is purified on a CombiFlash Rf+ automated chromatography instrument using a mixture of ethyl acetate in hexanes. 


\subsection{Reaction products and characterization data}<smiles>Cc1ccc(-c2ccccc2)cc1</smiles>

4-Methyl-1,1'-biphenyl (3) was prepared according to general procedure A. Purification was performed using a gradient of $1 \rightarrow 5 \%$ ethyl acetate in hexanes to afford ( 3 ) as a yellow liquid ( $30 \mathrm{mg}, 86 \%$ yield). The reaction was repeated according to general procedure B (24 mg, 73\% yield). Characterization data matched those previously reported. ${ }^{13}{ }^{1} \mathrm{H}$ NMR $\left(400 \mathrm{MHz}, \mathrm{CDCl}_{3}\right): \delta 7.62-7.60(\mathrm{~d}, J=7.8 \mathrm{~Hz}, 2 \mathrm{H}), 7.54-7.42$ (t, $J=8.4 \mathrm{~Hz}, 2 \mathrm{H}), 7.37-7.33(\mathrm{~d}, J=7.8 \mathrm{~Hz}, 2 \mathrm{H}), 7.29-7.27(\mathrm{~d}, J=7.8 \mathrm{~Hz}, 2 \mathrm{H}), 7.25(\mathrm{~m}, 1 \mathrm{H}) 2.47(\mathrm{~s}, 3 \mathrm{H}) ;{ }^{13} \mathrm{C}$ NMR $\left(100 \mathrm{MHz}, \mathrm{CDCl}_{3}\right): 141.1,138.3,136.9,129.4,127.6,126.9,126.8,126.9,21.0$.<smiles>Cc1ccc2ccccc2c1</smiles>

2-Methylnaphthalene (4) was prepared according to general procedure A. Purification was performed using a gradient of $1 \rightarrow 5 \%$ ethyl acetate in hexanes to afford (4) as an off-white solid ( $26 \mathrm{mg}$, $76 \%$ yield). The reaction was repeated according to general procedure B (22 mg, 70\% yield). Characterization data matched those previously reported. ${ }^{14}{ }^{1} \mathrm{H}$ NMR $\left(400 \mathrm{MHz}, \mathrm{CDCl}_{3}\right): \delta 7.80-7.78(\mathrm{~d}, J=8.0 \mathrm{~Hz}, 3 \mathrm{H}), 7.79(\mathrm{~m}$, 1H), 7.45-7.41 (m, 2H), 7.37-7.34 (d, J = 8.4 Hz, 1H), $2.51(\mathrm{~s}, 3 \mathrm{H}) ;{ }^{13} \mathrm{C}$ NMR (100 MHz, $\left.\mathrm{CDCl}_{3}\right):$ 135.4, 133.6, $131.7,128.0,127.6,127.5,127.1,126.0,125.7,124.9,21.6$.<smiles>Cc1cc2ccccc2c2ccccc12</smiles>

9-Methylphenanthrene (5) was prepared according to general procedure A. Purification was performed using a gradient of $1 \rightarrow 5 \%$ ethyl acetate in hexanes to afford (5) as a faint yellow solid ( $32 \mathrm{mg}, 73 \%$ yield). Characterization data matched those previously reported. ${ }^{15} \quad{ }^{1} \mathrm{H}$ NMR $\left(400 \mathrm{MHz}, \mathrm{CDCl}_{3}\right): \delta$ 8.77-8.67 (m, 3H), 8.11-8.09 (m, 1H), 7.94-7.57 (m, 5H), $\left.2.77(\mathrm{~s}, 3 \mathrm{H}) ;{ }^{13} \mathrm{C} \mathrm{NMR} \mathrm{(100} \mathrm{MHz,} \mathrm{CDCl}_{3}\right):$ 141.2, 138.4, 137.0, $129.5,128.7,127.0,127.0,21.1$. 


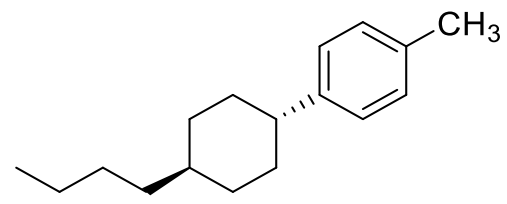

1-(trans-4-Butylcyclohexyl)-4-methylbenzene (6) was prepared according to general procedure A. Purification was done using a gradient of $1 \rightarrow 5 \%$ EtOAc in hexane to afford (6) as colourless liquid (35 mg, $76 \%$ yield). ${ }^{1} \mathrm{H}$ NMR $\left(400 \mathrm{MHz}, \mathrm{CDCl}_{3}\right): \delta$ 7.11-7.10 (m, 4H), 2.46-2.39 (m, 1H), $2.32(\mathrm{~s}, 3 \mathrm{H}), 1.90-1.85(\mathrm{~m}$, $4 \mathrm{H}), 1.49-1.38(\mathrm{~m}, 2 \mathrm{H}), 1.34-1.21(\mathrm{~m}, 7 \mathrm{H}), 1.10-1.00(\mathrm{~m}, 2 \mathrm{H}), 0.93-0.89(\mathrm{~m}, 3 \mathrm{H}) ;{ }^{13} \mathrm{C} \mathrm{NMR}\left(100 \mathrm{MHz}, \mathrm{CDCl}_{3}\right)$ : 144.9, 135.1, 128.9, 126.7, 44.2, 37.3, 37.1, 34.4, 33.7, 29.2, 23.0, 20.9, 14.1; Accurate mass (EI): Theoretical: 230.2035 . Found: 230.2029 . Spectral Accuracy: $98.5 \%$. Melting Point: $164-167^{\circ} \mathrm{C}$.

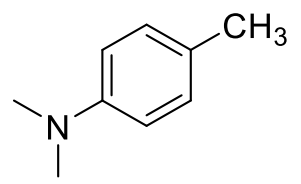

$\mathbf{N}, \mathbf{N}, \mathbf{4}-$ Trimethylaniline (7) was prepared according to general procedure A. Purification was performed using a gradient of $1 \rightarrow 15 \%$ ethyl acetate in hexanes to afford ( 7 ) as a yellow liquid ( $28 \mathrm{mg}, 73 \%$ yield). The reaction was repeated in a $50 \mathrm{~mL}$ heavy-walled pressure tube using $5.58 \mathrm{mmol}(1.0 \mathrm{~g})$ methyl 4(dimethylamino)benzoate, $11.2 \mathrm{mmol}\left(1.97 \mathrm{~mL}\right.$ ) TMDSO, $5.58 \mathrm{mmol}(626 \mathrm{mg}) \mathrm{KO}{ }^{\mathrm{t}} \mathrm{Bu}, 0.167 \mathrm{mmol}(46.0$ $\mathrm{mg}) \mathrm{Ni}(\mathrm{cod})_{2}$ and $0.335 \mathrm{mmol}(107 \mathrm{mg}) \mathrm{ICy} \cdot \mathrm{HBF}_{4}$ in $22 \mathrm{~mL}$ toluene - purification was performed using column chromatography with a gradient of $1 \rightarrow 15 \%$ ethyl acetate in hexanes to afford (7) as a white solid (761 mg, 77\% yield). Characterization data matched those previously reported. ${ }^{16}{ }^{1} \mathbf{H}$ NMR (400 MHz, $\left.\mathrm{CDCl}_{3}\right): \delta$ 7.07-7.05 (d, $\left.J=8.8 \mathrm{~Hz}, 2 \mathrm{H}\right), 6.79-6.68(\mathrm{~d}, J=8.8 \mathrm{~Hz}, 2 \mathrm{H}), 2.92(\mathrm{~s}, 6 \mathrm{H}), 2.26(\mathrm{~s}, 3 \mathrm{H}) ;{ }^{13} \mathrm{C}$ NMR $(100$ $\left.\mathrm{MHz}, \mathrm{CDCl}_{3}\right):$ 129.5, 129.2, 113.3, 41.1, 21.2, 21.1 .

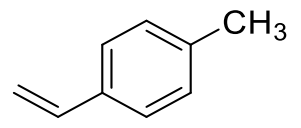

1-Methyl-4-vinylbenzene (8) was prepared according to a modified general procedure A using 1.5 equivalents of TMDSO, a reaction temperature of $90{ }^{\circ} \mathrm{C}$ and a reaction time of $6 \mathrm{~h}$. Purification was performed using a gradient of $1 \rightarrow 5 \%$ ethyl acetate in hexanes to afford (8) as a colourless liquid ( $26 \mathrm{mg}$, 
$74 \%$ yield). Characterization data matched those previously reported. ${ }^{17}{ }^{1} \mathrm{H}$ NMR $\left(400 \mathrm{MHz}, \mathrm{CDCl}_{3}\right): \delta 7.38$ $7.36(\mathrm{~d}, J=8.2 \mathrm{~Hz}, 2 \mathrm{H}), 7.24-7.21(\mathrm{~d}, J=8.0 \mathrm{~Hz}, 2 \mathrm{H}), 6.79-6.72(\mathrm{dd}, J=18.0,11.4 \mathrm{~Hz}, 1 \mathrm{H}), 5.78-5.74(\mathrm{dd}, J=$ 18.0, $1.4 \mathrm{~Hz}, 1 \mathrm{H}), 5.26-5.23(\mathrm{dd}, J=10.8,1.4 \mathrm{~Hz}, 1 \mathrm{H}), 2.40(\mathrm{~s}, 3 \mathrm{H}) ;{ }^{13} \mathrm{C} \mathrm{NMR}\left(100 \mathrm{MHz}, \mathrm{CDCl}_{3}\right): 137.6,136.7$, $134.9,129.2,126.1,112.7,21.2$.

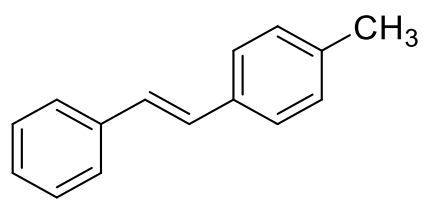

1-Methyl-4-(2-phenylethenyl)benzene (9) was prepared according to a modified general procedure A using 1.5 equivalents of TMDSO, a reaction temperature of $90^{\circ} \mathrm{C}$ and a reaction time of $6 \mathrm{~h}$. Purification was performed using a gradient of $1 \rightarrow 5 \%$ ethyl acetate in hexanes to afford (9) as a white solid (22 $\mathrm{mg}$, $71 \%$ yield). Characterization data matched those previously reported. ${ }^{18}{ }^{1} \mathrm{H} \mathrm{NMR}\left(400 \mathrm{MHz}, \mathrm{CDCl}_{3}\right): \delta 7.53-$ $7.51(\mathrm{~m}, 1 \mathrm{H}), 7.51-7.50(\mathrm{~m}, 1 \mathrm{H})$, 7.36-7.28 $(\mathrm{m}, 4 \mathrm{H}), 7.26-7.24(\mathrm{~m}, 3 \mathrm{H}), 7.18-7.11(\mathrm{~m}, 2 \mathrm{H}), 2.35(\mathrm{~s}, 3 \mathrm{H}) ;{ }^{13} \mathrm{C}$ NMR $\left(100 \mathrm{MHz} \mathrm{CDCl}_{3}\right): 131.1,130.0,129.0,128.9,128.7,128.3,127.5,126.7,126.3,125.3,21.4$.<smiles>COc1cc(C)cc(OC)c1OC</smiles>

1,2,3-Trimethoxy-5-methylbenzene (10) was prepared according to general procedure A. Purification was done using a gradient of $1 \rightarrow 5 \%$ EtOAc in hexane to afford (10) as colourless liquid $(25 \mathrm{mg}, 69 \%$ yield). Characterization data matched those previously reported. ${ }^{19}{ }^{1} \mathrm{H}$ NMR $\left(400 \mathrm{MHz}, \mathrm{CDCl}_{3}\right): \delta 6.40$ (s, 2H), 3.82-3.79 (m, 9H), 2.29 (s, 3H); $\left.{ }^{13} \mathrm{C} \mathrm{NMR} \mathrm{(100} \mathrm{MHz,} \mathrm{CDCl}_{3}\right): 152.9,135.7,133.5,105.9,60.8,55.9$, 21.8 . 
1-Methyl-4-phenoxybenzene (11) was prepared according to general procedure A. Purification was performed using a gradient of $1 \rightarrow 5 \%$ EtOAc in hexane to afford (11) as colorless solid ( $28 \mathrm{mg}, 71 \%$ yield). Characterization data matched those previously reported. ${ }^{20}{ }^{1} \mathrm{H}$ NMR $\left(400 \mathrm{MHz}, \mathrm{CDCl}_{3}\right): \delta 7.41$ (t, J=8.3 $\mathrm{Hz}, 2 \mathrm{H}), 7.22$ (d, J = 8.4 Hz, 2H), 7.13-7.10 (m, 3H), 6.91-6.88 (d, J=8.0 Hz, 2H), $2.33(\mathrm{~s}, 3 \mathrm{H}) ;{ }^{13} \mathrm{C} \mathrm{NMR}(100$ $\left.\mathrm{MHz}, \mathrm{CDCl}_{3}\right): 158.8,155.3,132.4,130.1,129.8,123.2,120.4,118.6,20.7$.<smiles>COc1ccc(Oc2ccc(C)cc2)cc1</smiles>

1-Methoxy-4-( $\boldsymbol{p}$-tolyloxy)benzene (12) was prepared according to general procedure A. Purification was performed using a gradient of $1 \rightarrow 5 \%$ EtOAc in hexane to afford (12) as colourless liquid ( $26 \mathrm{mg}, 63 \%$ yield). Characterization data matched those previously reported. ${ }^{20}{ }^{1} \mathrm{H}$ NMR $\left(400 \mathrm{MHz}, \mathrm{CDCl}_{3}\right): \delta 7.10-7.07(\mathrm{~d}, J=$ $8.2 \mathrm{~Hz}, 2 \mathrm{H}), 6.96-6.92(\mathrm{~d}, J=8.8 \mathrm{~Hz}, 2 \mathrm{H}), 6.87-6.82(\mathrm{~m}, 4 \mathrm{H}), 3.78(\mathrm{~s}, 3 \mathrm{H}), 2.30(\mathrm{~s}, 3 \mathrm{H}) ;{ }^{13} \mathrm{C}$ NMR $(100 \mathrm{MHz}$, $\left.\mathrm{CDCl}_{3}\right): 156.0,155.6,150.7,132.0,130.0,120.3,117.8,114.7,55.6,20.6$.<smiles>Cc1ccc(Oc2ccc(Oc3ccccc3)cc2)cc1</smiles>

1-Methyl-4-(4-phenoxyphenoxy)benzene (13) was prepared according to general procedure A. Purification was performed using a gradient of $1 \rightarrow 5 \%$ EtOAc in hexane to afford (13) as a colourless liquid (23 mg, 72\% yield). The reaction was repeated according to general procedure B (17 mg, 64\% yield). Characterization data matched those previously reported. ${ }^{20}{ }^{1} \mathbf{H}$ NMR $\left(400 \mathrm{MHz}, \mathrm{CDCl}_{3}\right): \delta$ 7.34-7.29 (m, 2H), 7.14-7.05 (m, 2H), $7.05(\mathrm{t}, J=1.2 \mathrm{~Hz}, 1 \mathrm{H}), 7.01-6.95(\mathrm{~m}, 6 \mathrm{H}), 6.93-6.89(\mathrm{~m}, 2 \mathrm{H}), 2.33(\mathrm{~s}, 3 \mathrm{H}) ;{ }^{13} \mathrm{C} \mathrm{NMR}$ $\left(100 \mathrm{MHz}, \mathrm{CDCl}_{3}\right): 157.9,155.2,153.3,152.3,132.6,130.2,129.7,122.9,120.4,119.9,118.5,118.1,20.7$. 
<smiles>Cc1ccc(-c2ccccc2O)cc1</smiles>

4'-Methyl-[1,1'-biphenyl]-2-ol (14) was prepared according to general procedure A. After the TBAF workup, the reaction solution was quenched with $1.0 \mathrm{M} \mathrm{HCl}$. A liquid-liquid extraction was performed with $2 \times 5 \mathrm{~mL}$ of ethyl acetate - the resulting organic fractions were collected and solvent was evaporated via rotary evaporation. The subsequent crude mixture was redissolved in $0.8 \mathrm{~mL}$ of dichloromethane and purified on a Combiflash Rf automated chromatography instrument using a gradient of $1 \rightarrow 5 \%$ EtOAc in hexane to afford (14) as yellow liquid (19 mg, 66\% yield). Characterization data matched those previously reported. ${ }^{21}{ }^{1} \mathrm{H}$ NMR (400 MHz, $\left.\mathrm{CDCl}_{3}\right): \delta$ 7.35-7.32 (m, 2H), 7.29-7.27 (m, 2H), 7.25-7.19 (m, 2H), 6.98$6.94(\mathrm{~m}, 2 \mathrm{H}), 5.18$ (s, 1H), 2.39 (s, 3H); ${ }^{13} \mathrm{C}$ NMR (100 MHz, CDCl 3$)$ : 152.4, 137.7, 133.9, 130.1, 130.0, 129.0, $128.9,128.0,120.7,115.6,21.2$.

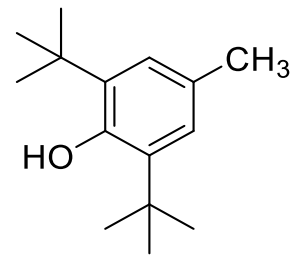

2,6-Bis(1,1-dimethylethyl)-4-methylphenol (15) was prepared according to the general procedure A, modified to use a reaction temperature of $90{ }^{\circ} \mathrm{C}$ and a reaction time of $6 \mathrm{~h}$. After the TBAF workup, the reaction solution was quenched with $1.0 \mathrm{M} \mathrm{HCl}$. A liquid-liquid extraction was performed with $2 \times 5 \mathrm{~mL}$ of ethyl acetate - the resulting organic fractions were collected and solvent was evaporated via rotary evaporation. The subsequent crude mixture was dissolved in $0.8 \mathrm{~mL}$ of dichloromethane and purified on a Combiflash Rf automated chromatography instrument using a gradient of $1 \rightarrow 5 \%$ EtOAc in hexanes to afford (15) as an off-white solid (23 $\mathrm{mg}, 81 \%$ yield). The reaction was repeated according to general procedure $B\left(15 \mathrm{mg}, 67 \%\right.$ yield). Characterization data matched those previously reported. ${ }^{22}{ }^{1} \mathbf{H}$ NMR (400 $\left.\mathrm{MHz}, \mathrm{CDCl}_{3}\right): \delta 7.06(\mathrm{~s}, 2 \mathrm{H}), 5.08(\mathrm{~s}, 1 \mathrm{H}), 2.36(\mathrm{~s}, 3 \mathrm{H}), 1.52(\mathrm{t}, J=7.8 \mathrm{~Hz} ; 0.8 \mathrm{~Hz}, 18 \mathrm{H}) ;{ }^{13} \mathrm{C} \mathrm{NMR}(100 \mathrm{MHz}$, $\left.\mathrm{CDCl}_{3}\right): 151.6,135.8,128.3,125.6,34.3,30.4 .21 .3$. 
<smiles>Cc1ccc(-c2ccc(C(=O)O)cc2)cc1</smiles>

4'-Methyl-4-biphenylcarboxylic acid (16) was prepared according to a modified general procedure A, employing 2.2 equivalents of $\mathrm{KO}^{t} \mathrm{Bu}$ rather than 1.0 equivalent. After the TBAF workup, the reaction solution was quenched with $1.0 \mathrm{M} \mathrm{HCl}$. A liquid-liquid extraction was performed with $2 \times 5 \mathrm{~mL}$ of ethyl acetate - the resulting organic fractions were collected and solvent was evaporated via rotary evaporation. The subsequent crude mixture was redissolved in $0.8 \mathrm{~mL}$ of dichloromethane and purified on a Combiflash Rf automated chromatography instrument using a gradient of $10 \rightarrow 45 \%$ EtOAc in hexane to afford (16) as an off-white solid (29 $\mathrm{mg}, 82 \%$ yield). Characterization data matched those previously reported..$^{52}{ }^{1} \mathrm{H}$ NMR $\left(400 \mathrm{MHz}\right.$, DMSO- $\left.d_{6}\right): \delta 8.00-7.97(\mathrm{~m}, 2 \mathrm{H}), 7.77-7.68(\mathrm{~m}, 2 \mathrm{H})$, 7.64-7.51 $(\mathrm{m}, 2 \mathrm{H})$, 6.97$6.95(\mathrm{~m}, 2 \mathrm{H}), 2.21$ (s, 3H); ${ }^{13} \mathrm{C}$ NMR (100 MHz, DMSO- $\left.d_{6}\right): 166.9,141.7,140.3,138.1,132.3,130.4,129.9$, 127.4, 127.1, 20.4. Accurate mass (EI): Theoretical: 212.0804. Found: 212.0794. Spectral Accuracy: 96.8\%. FT-IR: $v\left(\mathrm{~cm}^{-1}\right) 2953,2901,1678,1611,1420,1326,1291 . \mathrm{mp}: 241-244{ }^{\circ} \mathrm{C}$.<smiles>Cc1ccc(-c2ccccc2)nc1</smiles>

5-Methyl-2-phenylpyridine (17) was prepared according to general procedure A. Purification was performed using a gradient of $1 \rightarrow 5 \%$ EtOAc in hexane to afford (17) as colorless solid ( $29 \mathrm{mg}, 73 \%$ yield). Characterization data matched those previously reported. ${ }^{24}{ }^{1} \mathrm{H}$ NMR $\left(400 \mathrm{MHz}, \mathrm{CDCl}_{3}\right): \delta 8.50(\mathrm{~d}, J=2.0$ $\mathrm{Hz}, 1 \mathrm{H})$, 7.95-7.92 (m, 2H), 7.62-7.60 (m, 1H), 7.55-7.53 (m, 1H), 7.46-7.41 (m, 2H), 7.39-7.34 (m, 1H), 2.35 (s, 3H); 13C NMR (100 MHz, $\left.\mathrm{CDCl}_{3}\right): 154.7,150.0,139.3,137.4,131.7,128.7,128.6,126.7,120.1,18.2$.

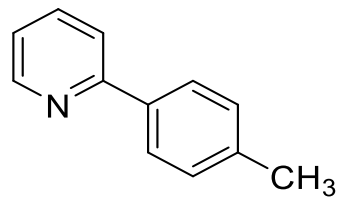


2-(p-Tolyl)pyridine (18) was prepared according to the general procedure A. Purification was performed using a gradient of $1 \rightarrow 5 \%$ EtOAc in hexane to afford (18) as colorless liquid (26 $\mathrm{mg}, 78 \%$ yield). Characterization data matched those previously reported. ${ }^{25}{ }^{1} \mathrm{H}$ NMR $\left(400 \mathrm{MHz}, \mathrm{CDCl}_{3}\right): \delta 8.67-8.65(\mathrm{~d}, J=$ 8.4 Hz, 1H), 7.89-7.86 (m, 2H), 7.77-7.67 (m, 2H), 7.27-7.24 (d, J = 8.0 Hz, 2H), 7.19-7.16 (m, 1H), 2.39 (s, 3H); ${ }^{13}$ C NMR (100 MHz, $\left.\mathrm{CDCl}_{3}\right): 157.4,149.5,138.9,136.7,136.6,129.4,126.7,121.7,120.2,21.2$.

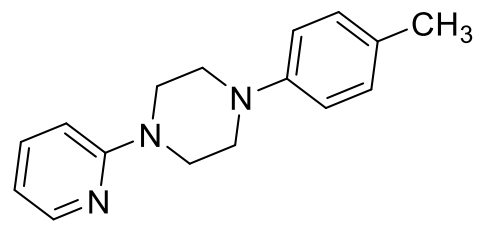

1-(4-Methylphenyl)-4-phenylpiperazine (19) was prepared according to general procedure A. Purification was performed using a gradient of $1 \rightarrow 5 \%$ ethyl acetate in hexanes to afford (19) as a white solid ( $28 \mathrm{mg}$, $67 \%$ yield). Characterization data matched those previously reported. ${ }^{26}{ }^{1} \mathbf{H ~ N M R}\left(400 \mathrm{MHz}, \mathrm{CDCl}_{3}\right): \delta 8.20$ $(\mathrm{m}, 1 \mathrm{H})$, 7.51-7.47 (m, 1H), 7.10-7.07 (m, 1H), 6.90-6.88 (m, 2H), 6.70-6.62 (m, 3H), 3.70-3.68 (m, 4H), 3.25-3.21 (m, 4H), 2.27 (s, 3H) ; $\left.{ }^{13} \mathrm{C} \mathrm{NMR} \mathrm{(100} \mathrm{MHz,} \mathrm{CDCl}\right): 159.4,149.2,147.9,137.5,129.7,116.7,113.5$, $107.2,49.8,45.3,30.9,20.4$.

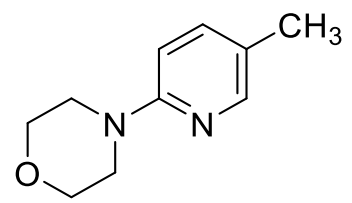

4-(5-Methyl-2-pyridinyl)-morpholine (20) was prepared according to general procedure A. Purification was performed using a gradient of $1 \rightarrow 5 \%$ ethyl acetate in hexanes to afford (20) as a white solid (28 $\mathrm{mg}$, $78 \%$ yield). Characterization data matched those previously reported. ${ }^{26}{ }^{1} \mathbf{H ~ N M R}\left(400 \mathrm{MHz}, \mathrm{CDCl}_{3}\right): \delta 8.02$ $(\mathrm{m}, 1 \mathrm{H}), 7.35-7.32(\mathrm{~m}, 1 \mathrm{H}), 6.68-6.56(\mathrm{~m}, 1 \mathrm{H}), 3.81(\mathrm{~m}, 4 \mathrm{H}), 3.43(\mathrm{~m}, 4 \mathrm{H}), 2.20(\mathrm{~s}, 3 \mathrm{H}) ;{ }^{13} \mathrm{C}$ NMR (100 MHz, $\left.\mathrm{CDCl}_{3}\right): 158.1,147.5,138.4,122.8,106.8,68.7,46.1,17.1$.

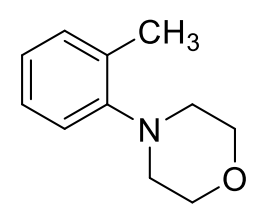


4-(2-Methylphenyl)-morpholine (21) was prepared according to general procedure A. Purification was performed using a gradient of $1 \rightarrow 5 \%$ ethyl acetate in hexanes to afford (21) as a white solid ( $25 \mathrm{mg}, 73 \%$ yield). Characterization data matched those previously reported. ${ }^{27}{ }^{1} \mathrm{H}$ NMR $\left(400 \mathrm{MHz}, \mathrm{CDCl}_{3}\right): \delta 7.21-7.18$ $(\mathrm{m}, 2 \mathrm{H}), 7.05-6.98(\mathrm{~m}, 2 \mathrm{H}), 3.87-3.85(\mathrm{~m}, 4 \mathrm{H}), 2.93-2.91(\mathrm{~m}, 4 \mathrm{H}), 2.33(\mathrm{~s}, 3 \mathrm{H}) ;{ }^{13} \mathrm{C} \mathrm{NMR}\left(100 \mathrm{MHz}, \mathrm{CDCl}_{3}\right)$ : 167.0, 153.9, 138.4, 137.6, 131.2, 120.1, 113.6, 107.1, 51.6, 47.2, 44.8 .

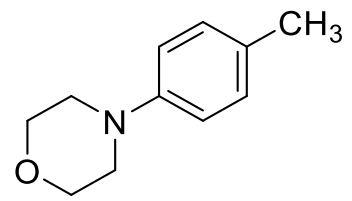

4-( $\boldsymbol{p}$-Tolyl)morpholine (22) was prepared according to general procedure A. Purification was done using a gradient of $1 \rightarrow 5 \%$ EtOAc in hexanes to afford (22) as off-white solid ( $27 \mathrm{mg}, 77 \%$ yield). The reaction was repeated according to general procedure $B(21 \mathrm{mg}, 71 \%$ yield). Characterization data matched those previously reported. ${ }^{27}{ }^{1} \mathrm{H} \mathrm{NMR}\left(400 \mathrm{MHz}, \mathrm{CDCl}_{3}\right): \delta 7.08(\mathrm{~d}, J=6.8 \mathrm{~Hz}, 2 \mathrm{H}), 6.83(\mathrm{~d}, J=8.4 \mathrm{~Hz}, 2 \mathrm{H}), 3.85(\mathrm{~m}$, 4H), $3.10(\mathrm{t}, J=4.8 \mathrm{~Hz}, 4 \mathrm{H}), 2.27(\mathrm{~s}, 3 \mathrm{H}) ;{ }^{13} \mathrm{C}$ NMR $\left(100 \mathrm{MHz}, \mathrm{CDCl}_{3}\right): 149.1,129.6,129.5,115.9,66.9,49.8$, 20.3 .

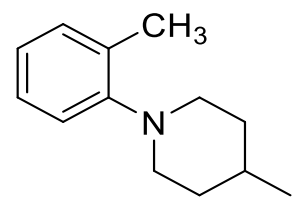

4-Methyl-1-(o-tolyl)piperidine (23) was prepared according to general procedure A. Purification was done a gradient of $1 \rightarrow 5 \%$ EtOAc in hexane to afford (23) as colorless liquid ( $24 \mathrm{mg}, 72 \%$ yield). Characterization data matched those previously reported. ${ }^{28}{ }^{1} \mathrm{H}$ NMR $\left(400 \mathrm{MHz}, \mathrm{CDCl}_{3}\right): \delta$ 7.16-7.11 (m, 2H), 7.00-6.91 (m, $2 \mathrm{H}), 3.09-3.06(\mathrm{t}, J=8.4 \mathrm{~Hz}, 2 \mathrm{H}), 2.64-2.57(\mathrm{dt}, J=8.6,3.4 \mathrm{~Hz}, 2 \mathrm{H}), 2.28(\mathrm{~s}, 3 \mathrm{H}), 1.72-1.69(\mathrm{~m}, 2 \mathrm{H}), 1.63-$ $1.58(\mathrm{~m}, 1 \mathrm{H})$ 1.41-1.33 (m, 2H), 0.99-0.96 (s, 3H); ${ }^{13} \mathrm{C}$ NMR (100 MHz, CDCl $)$ : 168.8, 152.9, 131.4, 123.8, $120.5,118.7,53.1,51.9,34.5,30.7,21.9$.

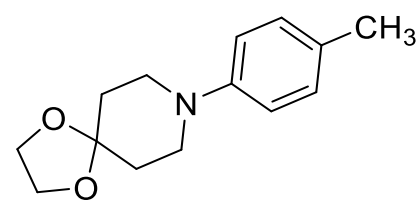


8-(4-Methylphenyl)-1,4-dioxa-8-azaspiro[4.5]decane (24) was prepared according to general procedure A. Purification was performed using a gradient of $1 \rightarrow 5 \%$ ethyl acetate in hexanes to afford (24) as a white solid (31 mg, 68\% yield). Characterization data matched those previously reported. ${ }^{29}{ }^{1} \mathbf{H}$ NMR (400 MHz, $\left.\mathrm{CDCl}_{3}\right):$ 7 7.07-7.06 (m, 2H), 6.89-6.87 (m, 2H), $3.99(\mathrm{~s}, 4 \mathrm{H}), 3.29-3.26(\mathrm{~m}, 4 \mathrm{H}), 2.28(\mathrm{~s}, 3 \mathrm{H}), 1.87-1.84(\mathrm{~m}$, 4H); ${ }^{13} \mathrm{C}$ NMR (100 MHz, $\left.\mathrm{CDCl}_{3}\right): 148.4,129.5,128.7,116.3,106.9,65.3,48.2,24.5,20.1$.

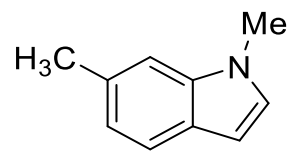

1,6-Dimethylindole (25) was prepared according to general procedure A. Purification was performed using a gradient of $1 \rightarrow 5 \%$ EtOAc in hexanes to afford (25) as colorless liquid ( $26 \mathrm{mg}, 79 \%$ yield). The reaction was repeated according to general procedure B (24 $\mathrm{mg}, 76 \%$ yield).Characterization data matched those previously reported. ${ }^{30}{ }^{1} \mathrm{H}$ NMR $\left(400 \mathrm{MHz}, \mathrm{CDCl}_{3}\right): \delta 7.55(\mathrm{dd}, J=8.0 \mathrm{~Hz}, 1.6 \mathrm{~Hz}, 1 \mathrm{H}), 7.15$ $(\mathrm{s}, 1 \mathrm{H}), 7.00-6.98(\mathrm{~m}, 2 \mathrm{H}), 6.48-6.46(\mathrm{~m}, 1 \mathrm{H}), 3.77(\mathrm{~s}, 3 \mathrm{H}) 2.54(\mathrm{~s}, 3 \mathrm{H}) ;{ }^{13} \mathrm{C}$ NMR $\left(100 \mathrm{MHz}, \mathrm{CDCl}_{3}\right): 137.0$, $131.1,128.1,126.2,121.0,120.4,109.1,100.6,32.6,21.8$.

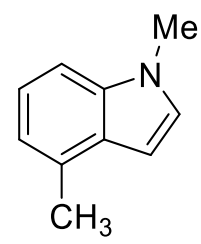

1,4-Dimethylindole (26) was prepared according to general procedure A. Purification was performed using a gradient of $1 \rightarrow 5 \%$ EtOAc in hexanes to afford (26) as colorless liquid (22 $\mathrm{mg}, 70 \%$ yield). Characterization data matched those previously reported. ${ }^{31}{ }^{1} \mathbf{H} \mathbf{N M R}\left(400 \mathrm{MHz}, \mathrm{CDCl}_{3}\right): \delta$ 7.18-7.16 (m, $2 \mathrm{H})$, 7.11-7.03 (d, J = 3.2 Hz, 1H), 6.92-6.89 (m, 1H), 6.50-6.49 (d, J = 3.2 Hz, 1H), 3.80 (s, 3H), $2.56(\mathrm{~s}, 3 \mathrm{H})$; ${ }^{13} \mathrm{C}$ NMR $\left(100 \mathrm{MHz}, \mathrm{CDCl}_{3}\right)$ : 136.4, 130.3, 128.36, 128.12, 121.6, 119.5, 106.8, 99.4, 33.0, 18.7.<smiles>Cc1cn(C)c2ccccc12</smiles> 
1,3-Dimethylindole (27) was prepared according to general procedure A. Purification was performed using a gradient of $1 \rightarrow 5 \%$ EtOAc in hexanes to afford (27) as colorless liquid (16 $\mathrm{mg}, 53 \%$ yield). Characterization data matched those previously reported. ${ }^{31}{ }^{1} \mathrm{H}$ NMR $\left(400 \mathrm{MHz}, \mathrm{CDCl}_{3}\right): \delta 7.65-7.62(\mathrm{~d}, J=$ $7.5 \mathrm{~Hz}, 1 \mathrm{H}), 7.34(\mathrm{~m}, 1 \mathrm{H}), 7.34-7.29(\mathrm{~m}, 1 \mathrm{H}), 7.21-7.18(\mathrm{t}, J=6.4 \mathrm{~Hz}, 1 \mathrm{H}), 6.87(\mathrm{~m}, 1 \mathrm{H}), 3.79(\mathrm{~s}, 3 \mathrm{H}), 2.41(\mathrm{~s}$, $3 \mathrm{H}) ;{ }^{13} \mathrm{C}$ NMR $\left(100 \mathrm{MHz}, \mathrm{CDCl}_{3}\right): 137.1,128.9,126.7,121.5,119.2,118.4,110.1,109.4,32.4,9.5$.

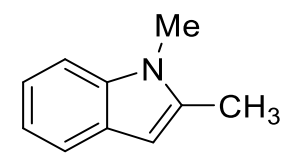

1,2-Dimethylindole (28) was prepared according to general procedure A. Purification was performed using a gradient of $1 \rightarrow 5 \%$ EtOAc in hexanes to afford (28) as colorless liquid (12 $\mathrm{mg}, 41 \%$ yield). Characterization data matched those previously reported. ${ }^{31}{ }^{1} \mathbf{H}$ NMR $\left(400 \mathrm{MHz}, \mathrm{CDCl}_{3}\right): \delta 7.61(\mathrm{~d}, J=7.8$ $\mathrm{Hz}, 1 \mathrm{H}), 7.34(\mathrm{~d}, J=5.4 \mathrm{~Hz}, 1 \mathrm{H}), 7.21-7.16(\mathrm{~m}, 2 \mathrm{H}), 6.34-6.31(\mathrm{~s}, 1 \mathrm{H}), 3.71(\mathrm{~s}, 3 \mathrm{H}), 2.43(\mathrm{~s}, 3 \mathrm{H}) ;{ }^{13} \mathrm{C}$ NMR (100 MHz, $\left.\mathrm{CDCl}_{3}\right): 137.4,136.8,127.8,120.4,119.7,119.4,108.7,99.7,29.5,12.7$.

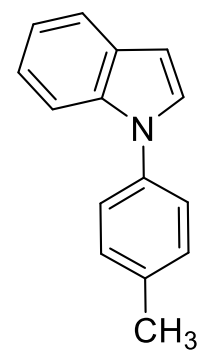

1-(p-Tolyl)-indole (29) was prepared according to general procedure A. Purification was performed using a gradient of $1 \rightarrow 5 \%$ EtOAc in hexane to afford (29) as yellow liquid ( $28 \mathrm{mg}, 67 \%$ yield). Characterization data matched those previously reported. ${ }^{32}{ }^{1} \mathrm{H}$ NMR $\left(400 \mathrm{MHz}, \mathrm{CDCl}_{3}\right): \delta 7.68$ (d, J=7.6 Hz, 1H), 7.53 (d, J $=8.0 \mathrm{~Hz}, 1 \mathrm{H}), 7.40-7.38(\mathrm{~m}, 2 \mathrm{H}), 7.32-7.30(\mathrm{~m}, 3 \mathrm{H}), 7.23-7.14(\mathrm{~m}, 2 \mathrm{H}), 6.67(\mathrm{~d}, J=3.2 \mathrm{~Hz}, 1 \mathrm{H}), 2.43(\mathrm{~s}, 3 \mathrm{H})$; ${ }^{13} \mathrm{C}$ NMR $\left(100 \mathrm{MHz}, \mathrm{CDCl}_{3}\right): 137.2,136.2,135.9,130.1,129.1,128.0,124.3,122.1,121.0,120.1,110.4$, 103.1, 21.0. 


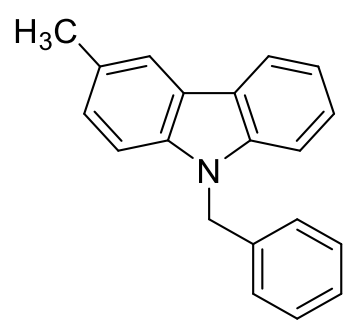

9-Benzyl-3-methylcarbazole (30) was prepared according to general procedure A. Purification was performed using a gradient of $1 \rightarrow 5 \%$ EtOAc in hexanes to afford (30) as white solid (39 $\mathrm{mg}, 72 \%$ yield). Characterization data matched those previously reported. ${ }^{33}{ }^{1} \mathrm{H}$ NMR $\left(400 \mathrm{MHz}, \mathrm{CDCl}_{3}\right): \delta 8.07(\mathrm{~d}, J=7.6$ $\mathrm{Hz}, 1 \mathrm{H}), 7.91(\mathrm{~s}, 1 \mathrm{H}), 7.41-7.36(\mathrm{~m}, 1 \mathrm{H}), 7.33-7.30(\mathrm{~m}, 1 \mathrm{H}), 7.25-7.18(\mathrm{~m}, 6 \mathrm{H}), 7.12-7.10(\mathrm{~m}, 2 \mathrm{H}), 5.48(\mathrm{~s}$, 2H), 2.52 (s, 3H); ${ }^{13} \mathrm{C}$ NMR (100 MHz, $\mathrm{CDCl}_{3}$ ): 140.8, 138.9, 137.3, 128.7, 128.5, 127.3, 127.1, 126.3, 125.6, $123.1,122.8,120.3,120.2,118.9,108.8,108.5,46.5,21.3$.

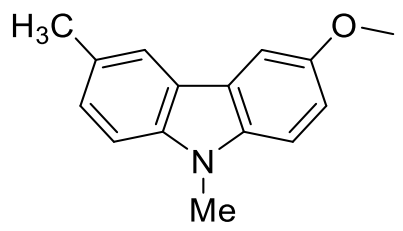

3-Methoxy-6,9-dimethyl-9H-carbazole (31) was prepared according to general procedure A. Purification was performed using a gradient of $1 \rightarrow 5 \%$ EtOAc in hexanes to afford (31) as off-white solid ( $29 \mathrm{mg}, 65 \%$ yield). Characterization data matched those previously reported. ${ }^{34}{ }^{1} \mathbf{H} \mathbf{N M R}\left(400 \mathrm{MHz}, \mathrm{CDCl}_{3}\right): \delta 7.84(\mathrm{~s}$, 1H), $7.55(\mathrm{~d}, J=2.4 \mathrm{~Hz}, 1 \mathrm{H}), 7.28-7.23(\mathrm{~m}, 3 \mathrm{H}), 7.09(\mathrm{dd}, J=8.8,2.4 \mathrm{~Hz}, 1 \mathrm{H}), 3.92(\mathrm{~s}, 2 \mathrm{H}), 3.77(\mathrm{~s}, 3 \mathrm{H}), 2.53$ (s, 3H); ${ }^{13} \mathrm{C}$ NMR $\left(100 \mathrm{MHz}, \mathrm{CDCl}_{3}\right): 153.4,139.9,136.4,127.5,127.0,122.8,122.6,120.1,114.6,109.0$, 108.2, 103.3, 56.1, 29.2, 21.3.

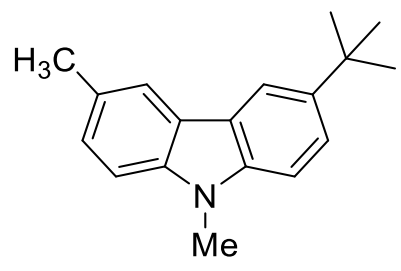

3-(tert-Butyl)-6,9-dimethyl-carbazole (32) was prepared according to general procedure A. Purification was performed using a gradient of $1 \rightarrow 5 \%$ EtOAc in hexanes to afford (32) as brown solid (35 mg, 68\% yield). Characterization data matched those previously reported. ${ }^{34}{ }^{1} \mathrm{H} \mathbf{N M R}\left(400 \mathrm{MHz}, \mathrm{CDCl}_{3}\right): \delta 8.06(\mathrm{~d}, J$ 
$=2.0 \mathrm{~Hz}, 1 \mathrm{H}), 7.89(\mathrm{~s}, 1 \mathrm{H}), 7.52(\mathrm{dd}, J=8.4,2.0 \mathrm{~Hz}, 1 \mathrm{H}), 7.29(\mathrm{~d}, J=8.8 \mathrm{~Hz}, 1 \mathrm{H}), 7.25(\mathrm{~s}, 2 \mathrm{H}), 3.79(\mathrm{~s}, 3 \mathrm{H})$, 2.53 (s, 3H), 1.43 (s, 9H); $\left.{ }^{13} \mathrm{C} \mathrm{NMR} \mathrm{(100} \mathrm{MHz,} \mathrm{CDCl}\right): 141.5,139.7,139.4,127.7,126.7,123.3,123.0,122.2$, $120.1,116.3,108.0,107.8,34.6,32.0,29.1,21.4$.

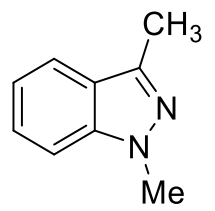

1,3-Dimethylindazole (33) was prepared according to general procedure A. Purification was performed using a gradient of $1 \rightarrow 5 \%$ EtOAc in hexanes to afford (33) as yellow liquid (17 $\mathrm{mg}, 59 \%$ yield). Characterization data matched those previously reported. ${ }^{35}{ }^{1} \mathrm{H}$ NMR $\left(400 \mathrm{MHz}, \mathrm{CDCl}_{3}\right): \delta 7.63$ (dt, $J=8.4$, $0.8 \mathrm{~Hz}, 1 \mathrm{H}), 7.37-7.28(\mathrm{~m}, 2 \mathrm{H}), 7.11-7.07(\mathrm{~m}, 1 \mathrm{H}), 3.98(\mathrm{~s}, 3 \mathrm{H}) 2.56(\mathrm{~s}, 3 \mathrm{H}) ;{ }^{13} \mathrm{C}$ NMR $\left(100 \mathrm{MHz}, \mathrm{CDCl}_{3}\right)$ : $141.2,140.8,126.1,123.2,120.3,119.5,108.7,35.0,11.8$.<smiles>Cc1ccc2ncccc2c1</smiles>

6-Methylquinoline (34) was prepared according to general procedure A. Purification was performed using a gradient of $1 \rightarrow 5 \%$ ethyl acetate in hexanes to afford (34) as a yellow-green oil ( $23 \mathrm{mg}, 63 \%$ yield). Characterization data matched those previously reported. ${ }^{23}{ }^{1} \mathrm{H} \mathrm{NMR}\left(400 \mathrm{MHz}, \mathrm{CDCl}_{3}\right): \delta 8.86(\mathrm{~m}, 1 \mathrm{H})$, 8.08-8.01 (m, 2H), 7.58-7.55 (m, 2H), 7.38-7.35 (m, 1H), $2.59(\mathrm{~s}, 3 \mathrm{H}) ;{ }^{13} \mathrm{C}$ NMR (100 MHz, $\left.\mathrm{CDCl}_{3}\right): 149.1$, $146.7,136.3,135.1,130.2,128.1,127.5,125.9,120.8,21.6$.

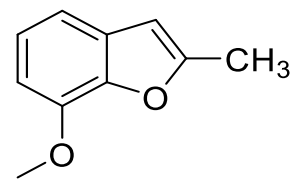

7-Methoxy-2-methylbenzofuran (35) was prepared according to a modified general procedure A using a reaction temperature of $90^{\circ} \mathrm{C}$ and a reaction time of $6 \mathrm{~h}$ Purification was done by CombiFlash column 
chromatography followed by preparatory TLC using a gradient of $1 \rightarrow 5 \%$ EtOAc in hexanes to afford (35) as colourless liquid $\left(21 \mathrm{mg}, 63 \%\right.$ yield). Characterization data matched those previously reported. ${ }^{36}{ }^{1} \mathbf{H}$ NMR $\left(400 \mathrm{MHz}_{\mathrm{CDCl}}\right.$ ): $\delta 7.10-7.04(\mathrm{~m}, 2 \mathrm{H}), 6.71(\mathrm{dd}, \mathrm{J}=7.2, \mathrm{~J}=1.6 \mathrm{~Hz}, 1 \mathrm{H}), 6.34(\mathrm{~s}, 1 \mathrm{H}), 3.98(\mathrm{~s}, 3 \mathrm{H}), 2.45$ (s, 3H); ${ }^{13} \mathrm{C}$ NMR $\left(100 \mathrm{MHz}, \mathrm{CDCl}_{3}\right):$ 155.5, 144.8, 143.7, 130.7, 123.0, 112.6, 105.3, 102.9, 55.9, 14.0.

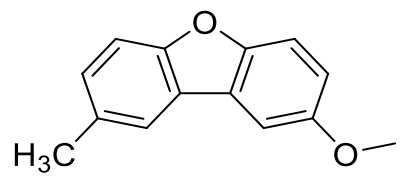

2-Methoxy-8-methyl-dibenzofuran (36) was prepared according to general procedure A. Purification was performed using a gradient of $1 \rightarrow 5 \%$ ethyl acetate in hexanes to afford (36) as a white solid ( $26 \mathrm{mg}, 81 \%$ yield). Characterization data matched those previously reported. ${ }^{37}{ }^{1} \mathbf{H ~ N M R}\left(400 \mathrm{MHz}, \mathrm{CDCl}_{3}\right): \delta 7.71-7.69$ $(\mathrm{s}, 2 \mathrm{H}), 7.46-7.38(\mathrm{~m}, 3 \mathrm{H}), 7.06-7.01(\mathrm{~m}, 1 \mathrm{H}), 3.91(\mathrm{~s}, 3 \mathrm{H}), 2.51(\mathrm{~s}, 3 \mathrm{H}) ;{ }^{13} \mathrm{C} \mathrm{NMR}\left(100 \mathrm{MHz}, \mathrm{CDCl}_{3}\right): 155.7$, $155.2,151.1,131.8,128.2,124.6,124.4,120.5,114.9,112.0,111.2,103.6,56.0,21.3$.<smiles>COc1cc(C)c(-c2c(C)cc(OC)c3c2OCO3)c2c1OCO2</smiles>

7,7'-Dimethoxy-5,5'-dimethyl-4,4'-bibenzo[d][1,3]dioxole (37) was prepared according to a modified general procedure $A$ using 4.0 equivalents of TMDSO and 2.0 equivalents of $\mathrm{KO}^{t} \mathrm{Bu}$. Purification was performed using a gradient of $1 \rightarrow 10 \%$ ethyl acetate in hexanes to afford (37) as a white solid (38 mg, 76\% yield). ${ }^{1} \mathrm{H}$ NMR $\left(400 \mathrm{MHz}, \mathrm{CDCl}_{3}\right): \delta$ 7.43-7.31 (m, 2H), 6.08-6.07 (m, 4H), $3.82(\mathrm{~s}, 6 \mathrm{H}), 2.51(\mathrm{~s}, 6 \mathrm{H}) ;{ }^{13} \mathrm{C} \mathrm{NMR}$ $\left(100 \mathrm{MHz}, \mathrm{CDCl}_{3}\right): 147.1,142.3,138.1,137.7,110.9,102.2,56.3,51.7,21.3$. Accurate mass (EI): Theoretical: 212.0832. Found: 212.0837. Spectral Accuracy: 96.3\%. FT-IR: $v\left(\mathrm{~cm}^{-1}\right)$ 1718, 1626, 1489, 1422, 1389, 1220, 1085, 1010. $\mathrm{mp:} 148-151^{\circ} \mathrm{C}$.<smiles>CCCN(CCC)S(=O)(=O)c1ccc(C)cc1</smiles> 
4-Methyl- $\mathbf{N}, \mathbf{N}$-dipropylbenzenesulfonamide (38) was prepared according to general procedure A. Purification was done using a gradient of $5 \rightarrow 10 \%$ EtOAc in hexane to afford (38) as yellow liquid (19 $\mathrm{mg}$, $36 \%$ yield). Characterization data matched those previously reported. ${ }^{39}{ }^{1} \mathrm{H}$ NMR $\left(400 \mathrm{MHz}, \mathrm{CDCl}_{3}\right): \delta 7.66$ $(\mathrm{d}, J=8.0 \mathrm{~Hz}, 2 \mathrm{H}), 7.26(\mathrm{dd}, J=8.4,0.2 \mathrm{~Hz}, 2 \mathrm{H}), 3.05-3.01(\mathrm{~m}, 4 \mathrm{H}), 1.57-1.47(\mathrm{~m}, 4 \mathrm{H}), 0.84(\mathrm{~m}, 6 \mathrm{H}) ;{ }^{13} \mathrm{C} \mathrm{NMR}$ $\left(100 \mathrm{MHz}, \mathrm{CDCl}_{3}\right): 142.8,137.2,129.5,127.0,50.0,22.0,21.4,11.1$.

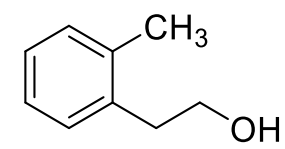

2-(Methylbenzene)ethanol (39) was prepared according to a modified general procedure A using 0.2 mmol of iso-chromanone as the starting material, a reaction temperature of $90^{\circ} \mathrm{C}$ and a reaction time of $6 \mathrm{~h}$. After the TBAF workup, the reaction solution was quenched with $1.0 \mathrm{M} \mathrm{HCl}$. A liquid-liquid extraction was performed with $2 \times 5 \mathrm{~mL}$ of ethyl acetate - the resulting organic fractions were collected and solvent was evaporated via rotary evaporation. The subsequent crude mixture was redissolved in $0.8 \mathrm{~mL}$ of dichloromethane and purified on a Combiflash Rf automated chromatography instrument using a gradient of $1 \rightarrow 5 \%$ EtOAc in hexanes to afford (39) as an off-white solid ( $21 \mathrm{mg}, 74 \%$ yield). Characterization data matched those previously reported. ${ }^{38}{ }^{1} \mathrm{H}$ NMR $\left(400 \mathrm{MHz}, \mathrm{CDCl}_{3}\right): \delta$ 7.29-7.17 (m, 4H), 3.71-3.66 (m, 2H), $3.22(\mathrm{~m}, 2 \mathrm{H}), 2.37(\mathrm{~s}, 3 \mathrm{H}) ;{ }^{13} \mathrm{C} \mathrm{NMR}\left(100 \mathrm{MHz}, \mathrm{CDCl}_{3}\right): 137.7,128.9,128.8,128.1,125.9,125.1,57.7,21.2$, 18.0. 


\section{Troubleshooting}

\subsection{Scope Limitations}

Several substrates, particularly those with free $\mathrm{NH}$ bonds, were found to be inert to the reaction conditions, instead giving recovery of starting material (Scheme S3A). Substrates with other carbonyl functionalities such as aldehydes and ketones were found to over-reduce to the corresponding methyl or methylene-containing products (Scheme S3B). Further, substrates bearing aliphatic esters, rather than aryl esters, were only found to reduce to the alcohol oxidation state (Scheme S3C). Lastly, esters bearing carbon-halogen bonds, carbon-sulfur bonds and select other functional groups were found to give intractable mixtures of products (Scheme S3D).

(A)<smiles>COC(=O)c1ccc2[nH]cnc2c1</smiles><smiles>COC(=O)c1ccc(NC(C)c2ccccn2)cc1</smiles><smiles>COC(=O)c1ccc(NC(=O)c2ccccc2)cc1</smiles><smiles>COC(=O)c1ccc(NCc2ccccc2)cc1</smiles><smiles>COC(=O)c1n[nH]c2ccccc12</smiles><smiles>COC(=O)c1ccc(-c2ccc(N)cc2)cc1</smiles>

(B)<smiles>COC(=O)c1ccc(-c2ccc(C=O)cc2)cc1</smiles><smiles>COC(=O)c1ccc(C=O)cc1</smiles><smiles>COC(=O)c1ccc(-c2ccc(C(C)=O)cc2)cc1</smiles><smiles>COC(=O)c1cccc(C(=O)c2ccccc2)c1</smiles>

(c)<smiles>COC(=O)CCCCc1ccccc1</smiles><smiles>CCCCCCCC/C=C/CCCCCCCC(=O)OC</smiles><smiles>COC(=O)Cc1ccc2ccccc2c1</smiles><smiles>COC(=O)C(C)c1ccc2cc(OC)ccc2c1</smiles><smiles>COC(=O)C(=O)CCc1ccccc1</smiles>

(D)

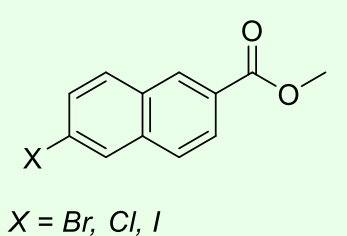<smiles>COC(=O)c1ccc(-c2ccc(I)cc2)cc1</smiles><smiles>COC(=O)c1ccc2cc(SC)ccc2c1</smiles><smiles>COC(=O)c1ccc(-c2ccccc2CC#N)cc1</smiles> 
Scheme S3. Unsuccessful scope examples

\subsection{Improving moderate yields}

Several substrates afforded relatively low yields when subjected to the 'optimal' conditions. In general, yields could be improved by increasing the catalyst loading. In many cases, substrate-specific optimization resulted in improved yields without necessitating more catalyst. For instance, Substrate $\mathbf{S 3}$, bearing a stilbene backbone, was found to give desired product 9 upon reaction with 1.5 equivalents TMDSO at 90 ${ }^{\circ} \mathrm{C}$ for $6 \mathrm{~h}$. Upon treatment with the general reaction conditions, product $\mathbf{S 4}$ could be recovered. Further, S4 could be recovered from substrate $\mathbf{S} 3$ upon reaction with 3 equivalents of TMDSO.

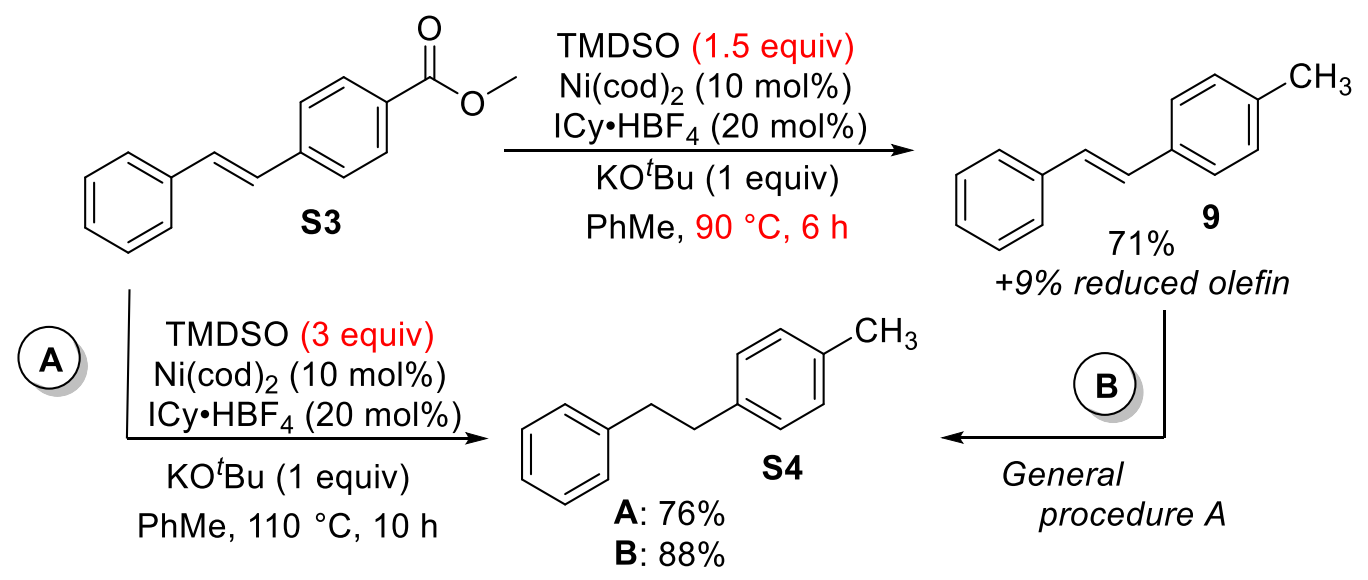

Scheme S4. Depicting control over olefin-containing ester substrates<smiles>Cc1ccc(CCc2ccccc2)cc1</smiles>

1-Methyl-4-(2-phenethyl)benzene (S4) was prepared according to general procedure A. Purification was performed using a gradient of $1 \rightarrow 5 \%$ ethyl acetate in hexanes to afford (S4) as a white solid (22 mg, 61\% yield). Characterization data matched those previously reported. ${ }^{40}{ }^{1} \mathrm{H}$ NMR $\left(400 \mathrm{MHz}, \mathrm{CDCl}_{3}\right): \delta 7.46-7.44$ $(\mathrm{d}, J=8.2 \mathrm{~Hz}, 2 \mathrm{H}), 7.34-7.32(\mathrm{~d}, J=8.0 \mathrm{~Hz}, 2 \mathrm{H}), 7.14-7.11(\mathrm{~m}, 3 \mathrm{H}), 7.01-6.93(\mathrm{~m}, 2 \mathrm{H}), 2.92-2.89(\mathrm{~m}, 4 \mathrm{H})$, 2.31 (s, 3H); ${ }^{13} \mathrm{C}$ NMR (100 MHz, $\left.\mathrm{CDCl}_{3}\right): 143.4,137.6,134.3,128.3,128.1,127.3,125.7,39.4,36.1$, 20.5. 
Substrate-specific optimization was performed on esters S5, S7, S8 and S9 after noting their modest yields upon exposure to the general procedure A. Higher yields could be obtained upon lowering the reaction temperature to $90{ }^{\circ} \mathrm{C}$ and shortening the reaction time to $6 \mathrm{~h}$.

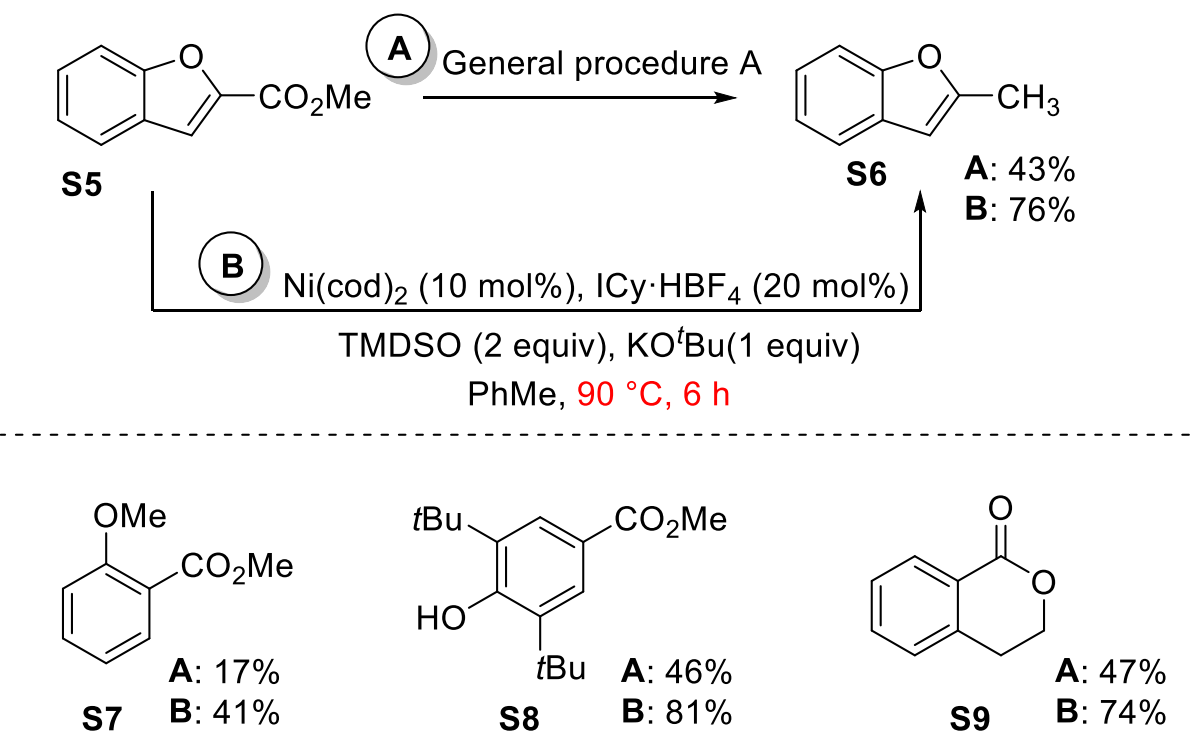

Scheme S5. Improved yields at reduced temperature and time 


\subsection{Employing a "weaker" reducing agent}

Products 40-45 were obtained upon substituting KF (1 eq) + $\mathrm{KO}^{t} \mathrm{Bu}(20 \mathrm{~mol} \%)$ for $\mathrm{KO} \mathrm{B}^{\mathrm{B} u}(1 \mathrm{eq})$. Under our original conditions, we tentatively propose that $20 \mathrm{~mol} \% \mathrm{KO}^{t} \mathrm{Bu}$ was used to deprotonate the ICy.HBF 4 while the remainder went towards the formation of an activated, hypervalent siloxane reducing agent. Under these modified conditions, we propose that the $\mathrm{KO}^{t} \mathrm{Bu}(20 \mathrm{~mol} \%)$ was used to deprotonate the $\mathrm{ICy} \cdot \mathrm{HBF}_{4}$ ligand while the $\mathrm{KF}$ was used to activate the siloxane species. Results obtained upon exposing substrates S10 and S11 bearing nitro groups and C-F bonds lead us to believe that combining TMDSO and $\mathrm{KF}$ creates a weaker reducing agent than does the combination of TMDSO and $\mathrm{KO}^{t} \mathrm{Bu}$.

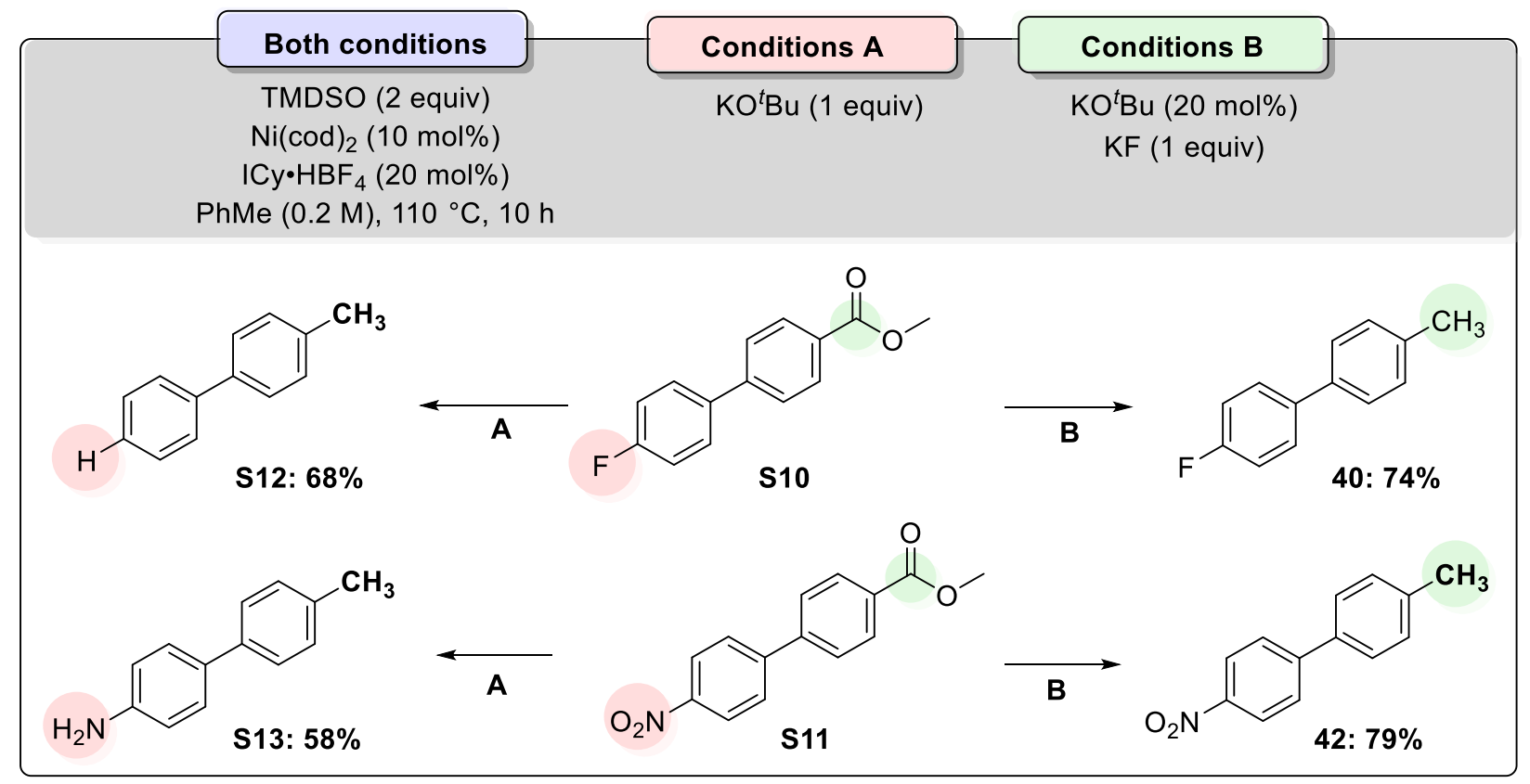

Scheme S6. Illustrating the deviations between using $\mathrm{KF}+\mathrm{KO}^{t} \mathrm{Bu}$ or using only $\mathrm{KO}{ }^{t} \mathrm{Bu}$<smiles>Cc1ccc(-c2ccc(F)cc2)cc1</smiles>

4-Fluoro-4'-methylbiphenyl (40) was prepared according to a modified general procedure $A$, employing 1.0 equivalent of $\mathrm{KF}$ and $20 \mathrm{~mol} \% \mathrm{KO}^{t} \mathrm{Bu}$ in place of 1.0 equivalent $\mathrm{KO}^{t} \mathrm{Bu}$. Purification was performed using a gradient of $1 \rightarrow 5 \%$ ethyl acetate in hexanes to afford (40) as a white solid ( $25 \mathrm{mg}, 74 \%$ yield). Characterization data matched those previously reported. ${ }^{53}{ }^{1} \mathrm{H}$ NMR $\left(400 \mathrm{MHz}, \mathrm{CDCl}_{3}\right): \delta 7.41-7.38(\mathrm{~m}$, 
$2 \mathrm{H}), 7.36-7.34(\mathrm{~m}, 2 \mathrm{H}), 7.30-7.28(\mathrm{~m}, 2 \mathrm{H}), 7.20-7.07(\mathrm{~m}, 2 \mathrm{H}), 2.30(\mathrm{~s}, 3 \mathrm{H}) ;{ }^{13} \mathrm{C} \mathrm{NMR}\left(100 \mathrm{MHz}, \mathrm{CDCl}_{3}\right): 161.9$, 161.7, 136.2, 130.3, 129.1, 128.2, 123.9, 115.4, 20.8. Accurate mass (EI): Theoretical: 186.0813. Found: 186.0816. Spectral Accuracy: $97.4 \% . \mathrm{mp:} 79-81^{\circ} \mathrm{C}$.

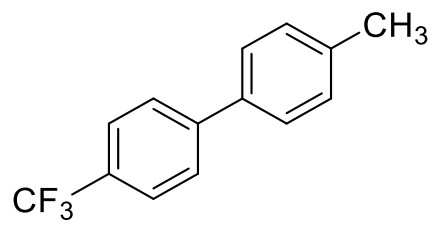

4-Methyl-4'-(trifluoromethyl)-1,1'-biphenyl (41) was prepared according to a modified general procedure $\mathrm{A}$, employing 1.0 equivalent of $\mathrm{KF}$ and $20 \mathrm{~mol} \% \mathrm{KO}^{t} \mathrm{Bu}$ in place of 1.0 equivalent $\mathrm{KO}^{t} \mathrm{Bu}$. Purification was performed using a gradient of $1 \rightarrow 25 \%$ ethyl acetate in hexanes to afford (41) as an offwhite solid ( $28 \mathrm{mg}, 84 \%$ yield). Characterization data matched those previously reported. ${ }^{54}{ }^{1} \mathbf{H}$ NMR (400 $\left.\mathrm{MHz}, \mathrm{CDCl}_{3}\right): \delta$ 7.66-7.54 (m, 2H), 7.49-7.47 (m, 2H), 7.45-7.42 (m, 2H), 7.38-7.36 (m, 2H), $2.41(\mathrm{~s}, 3 \mathrm{H}) ;{ }^{13} \mathrm{C}$ NMR $\left(100 \mathrm{MHz}, \mathrm{CDCl}_{3}\right): 142.1,138.2,136.8,129.2,128.7,126.8,125.9,124.3,123.9,21.4$. Accurate mass (El): Theoretical: 236.0846 . Found: 236.0839 . Spectral Accuracy: $94.6 \% . \mathrm{mp}: 119-122^{\circ} \mathrm{C}$.<smiles>Cc1ccc(-c2ccc([N+](=O)[O-])cc2)cc1</smiles>

4-Methyl-4'-nitrobiphenyl (42) was prepared according to a modified general procedure A, employing 1.0 equivalent of $\mathrm{KF}$ and $20 \mathrm{~mol} \% \mathrm{KO}^{\mathrm{t}} \mathrm{Bu}$ in place of 1.0 equivalent $\mathrm{KO}^{\mathrm{t}} \mathrm{Bu}$. Purification was performed using a gradient of $10 \rightarrow 45 \%$ ethyl acetate in hexanes to afford (42) as a white solid (26 $\mathrm{mg}, 79 \%$ yield). Characterization data matched those previously reported. ${ }^{55}{ }^{1} \mathbf{H}$ NMR $\left(400 \mathrm{MHz}, \mathrm{CDCl}_{3}\right): \delta$ 8.33-8.28 (m, $2 \mathrm{H}), 7.65-7.63(\mathrm{~m}, 2 \mathrm{H}), 7.55-7.48(\mathrm{~m}, 2 \mathrm{H}), 7.34-7.30(\mathrm{~m}, 2 \mathrm{H}), 2.44(\mathrm{~s}, 3 \mathrm{H}) ;{ }^{13} \mathrm{C} \mathrm{NMR}\left(100 \mathrm{MHz}, \mathrm{CDCl}_{3}\right): 147.6$, $138.8,129.9,128.7,127.8,127.4,124.1,21.2$. Accurate mass (EI): Theoretical: 213.0789 Found: 236.0806. Spectral Accuracy: $96.6 \% . \mathrm{mp}: 139-142^{\circ} \mathrm{C}$.

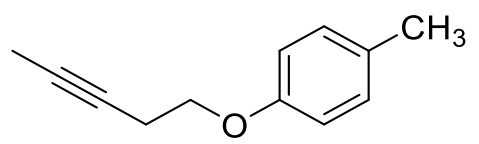

1-Methyl-4-(3-pentyn-1-yloxy)-benzene (43) was prepared according to a modified general procedure A, employing 1.0 equivalent of $\mathrm{KF}$ and $20 \mathrm{~mol} \% \mathrm{KO}^{t} \mathrm{Bu}$ in place of 1.0 equivalent $\mathrm{KO}^{t} \mathrm{Bu}$. Purification was performed using a gradient of $1 \rightarrow 25 \%$ ethyl acetate in hexanes to afford (43) as a white solid ( $20 \mathrm{mg}, 68 \%$ 
yield). ${ }^{1} \mathbf{H}$ NMR (400 MHz, $\left.\mathrm{CDCl}_{3}\right):$ 8 7.58-7.41 (m, 2H), 7.39-7.23 (m, 2H), 3.68-3.64 (m, 4H), 2.41-2.37 (m, 3H), $1.79(\mathrm{~m}, 3 \mathrm{H}) ;{ }^{13} \mathrm{C}$ NMR (100 MHz, $\left.\mathrm{CDCl}_{3}\right)$ : 140.7, 137.9, 136.6, 129.0, 128.3, 126.5, 75.3, 61.0, 22.6, 20.6, 3.0. Accurate mass (EI): Theoretical: 174.1340. Found: 174.1321 Spectral Accuracy: $97.1 \%$. mp: 121$124^{\circ} \mathrm{C}$.

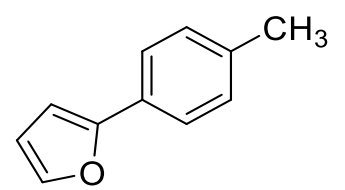

2-(4-Methylphenyl)furan (44) was prepared according to a modified general procedure A, employing 1.0 equivalent of $\mathrm{KF}$ and $20 \mathrm{~mol} \% \mathrm{KO}^{t} \mathrm{Bu}$ in place of 1.0 equivalent $\mathrm{KO}^{t} \mathrm{Bu}$. Purification was performed using a gradient of $1 \rightarrow 15 \%$ ethyl acetate in hexanes to afford (44) as a white solid (24 $\mathrm{mg}, 71 \%$ yield). Characterization data matched those previously reported. ${ }^{56}{ }^{1} \mathrm{H} \mathrm{NMR}\left(400 \mathrm{MHz}, \mathrm{CDCl}_{3}\right): \delta 7.59-7.57(\mathrm{~d}, \mathrm{~J}=$ $8.2 \mathrm{~Hz}, 2 \mathrm{H}), 7.48-7.37(\mathrm{~m}, 3 \mathrm{H}), 6.95-6.93(\mathrm{~m}, 1 \mathrm{H}), 6.47-6.46(\mathrm{~m}, 1 \mathrm{H}), 2.31(\mathrm{~s}, 3 \mathrm{H}) ;{ }^{13} \mathrm{C}$ NMR $(100 \mathrm{MHz}$, $\left.\mathrm{CDCl}_{3}\right): 143.5,140.1,137.4,137.3,137.2,131.2,131.2,113.4,99.5,90.2$, 20.1. FT-IR: $v\left(\mathrm{~cm}^{-1}\right) 3286,1634$, 1440, 1344, 1280, 1130, 1092, 1068, 630. Accurate mass (EI): Theoretical: 158.0731. Found: 158.0727. Spectral Accuracy: 95.6\%.

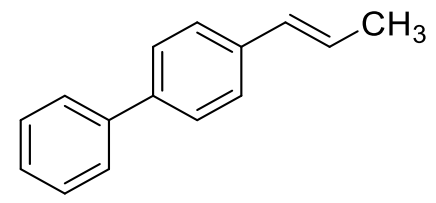

4-(1-Propen-1-yl)-1,1'-biphenyl (45) was prepared according to a modified general procedure A, employing 1.0 equivalent of $\mathrm{KF}$ and $20 \mathrm{~mol}^{\circ} \mathrm{KO}^{t} \mathrm{Bu}$ in place of 1.0 equivalent $\mathrm{KO}^{t} \mathrm{Bu}$. Purification was performed using a gradient of $1 \rightarrow 5 \%$ ethyl acetate in hexanes to afford (45) as an off-white solid (17 mg, $48 \%$ yield). Characterization data matched those previously reported. $.^{57}{ }^{1} \mathrm{H} \mathrm{NMR}\left(400 \mathrm{MHz}, \mathrm{CDCl}_{3}\right): \delta 7.58$ $7.54(\mathrm{~m}, 2 \mathrm{H}), 7.43-7.36(\mathrm{~m}, 2 \mathrm{H}), 7.24-7.18(\mathrm{~m}, 2 \mathrm{H}), 7.07-7.05(\mathrm{~m}, 3 \mathrm{H}), 6.32-6.28(\mathrm{~m}, 2 \mathrm{H}), 2.16(\mathrm{~d}, \mathrm{~J}=6.4$ $\mathrm{Hz}, 3 \mathrm{H}) ;{ }^{13} \mathrm{C}$ NMR $\left(100 \mathrm{MHz}, \mathrm{CDCl}_{3}\right):$ 144.8, 136.2, 134.4, 130.9, 130.3, 130.2, 128.8, 128.2, 128.0, 117.7, 18.8. Accurate mass (EI): Theoretical: 194.1124. Found: 194.1181. Spectral Accuracy: 96.2\%. mp: 90-94 ${ }^{\circ} \mathrm{C}$. 


\subsection{General comments}

The quality of reagents was observed to be particularly important for reproducibly obtaining the yields reported. In the conditions using $\mathrm{Ni}(\operatorname{cod})_{2}$, any exposure to oxygen was highly detrimental. The age of the $\mathrm{Ni}(\operatorname{cod})_{2}$ was also found to be important - in one instance, replacing an old (>6 month) bottle of Ni(cod) $)_{2}$ with a new source resulted in substantially improved yields. While somewhat lower yielding, reactions performed using general procedure $\mathrm{B}\left(\mathrm{NiBr}_{2} \cdot \mathrm{glyme} / \mathrm{Mn}\right)$ were found to be more robust. 


\section{Additional experimental information}

6.1._Catalytic deuteration

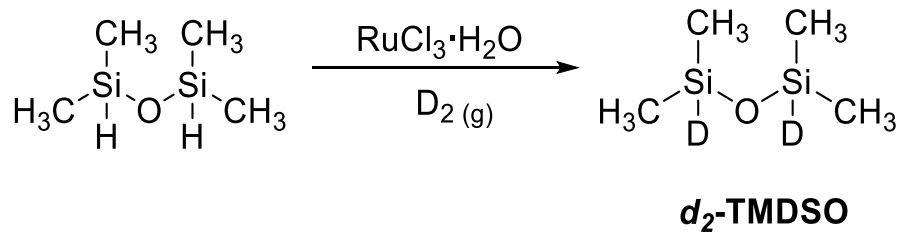

In order to transform TMDSO into its deuterated analog, a modified literature procedure was used. ${ }^{41}$ Inside of a nitrogen-filled glovebox, $10 \mathrm{mg} \mathrm{RuCl}{ }_{3} \cdot \mathrm{H}_{2} \mathrm{O}$ is crushed into a fine powder and added alongside $3.0 \mathrm{~mL}$ of TMDSO to a $25 \mathrm{~mL}$ heavy-walled vacuum Schlenk tube. The tube is connected to a Buchi "tinyclave" pressure reactor and sealed. The reaction vessel is subsequently brought outside of the glovebox and equipped with a deuterium gas regulator as well as to a vacuum pump via a T-shaped vacuum stopcock. The reaction vessel placed in a liquid nitrogen bath for 5 minutes, until it is frozen. It is subsequently placed under vacuum for 20 seconds, at which point the vacuum is removed and the reaction mixture is allowed to come to room temperature. After the mixture turns back into liquid, $D_{2}(\mathrm{~g})$ is introduced to the pressure tube to a gauge pressure of 10-12 psi. The reactor is sealed and stirred rapidly for $1 \mathrm{~h}$ at room temperature. After $1 \mathrm{~h}$, the reaction vessel is placed in a liquid nitrogen bath until the TMDSO solution freezes, at which point the vessel was placed under vacuum for 10 seconds. After evacuating, the pressure tube is brought out of the liquid nitrogen bath and allowed to return to room temperature. Once at room temperature, $D_{2}$ was reapplied at the same pressure. This cycle (pressurize vessel, stir, freeze-pump-thaw) was repeated 6 times, until analysis of an aliquot by ${ }^{1} \mathrm{H}$ NMR showed $>95 \%$ deuterium incorporation. The spectroscopically pure $d_{2}$-TMDSO (61\% yield) can be purified by distillation to remove remaining $\mathrm{RuCl}_{3} ;{ }^{41}$ however, this was not found to be necessary. The crude material was thus used in subsequent reduction reactions without further purification as a black, translucent liquid. 


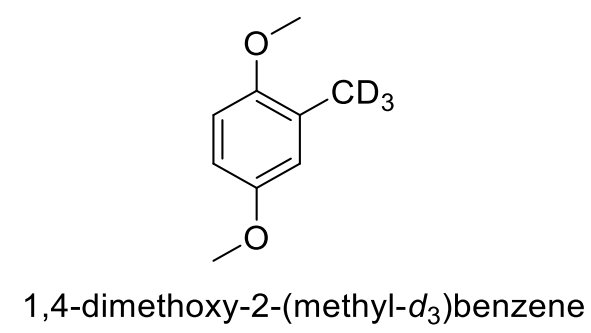

1,4-Dimethoxy-2-(methyl- $\left.\boldsymbol{d}_{3}\right)$ benzene (46) was prepared according to general procedure B using $d_{2^{-}}$ TMDSO in $d_{8}$-toluene (use of non-deuterated toluene provides slightly reduced deuterium incorporation). Purification was performed using a gradient of $1 \rightarrow 5 \%$ ethyl acetate in hexanes to afford (46) as a white solid (23 mg, 68\% yield, >95\% D). Characterization data matches the literature for the analogous nondeuterated compound. ${ }^{42}{ }^{1} \mathrm{H}$ NMR $\left(400 \mathrm{MHz}, \mathrm{CDCl}_{3}\right): \delta 6.69-6.59(\mathrm{~m}, 3 \mathrm{H}), 3.71(\mathrm{~s}, 3 \mathrm{H}), 3.67(\mathrm{~s}, 3 \mathrm{H}), 2.26(\mathrm{~s}$, $<0.08 \mathrm{H}) ;{ }^{13} \mathrm{C}$ NMR $\left(100 \mathrm{MHz}, \mathrm{CDCl}_{3}\right): 153.1,152.8,152.7,129.2,114.5,110.7,58.3,58.2,18.6\left(\mathrm{~m}_{1} \mathrm{CD}_{3}\right)$.

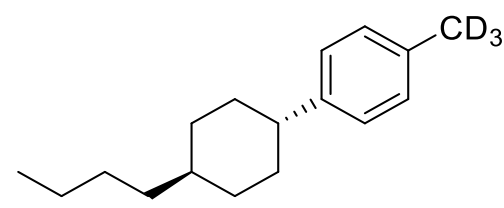

$\left(d_{3}\right)$-1-(trans-4-Butylcyclohexyl)-4-methylbenzene (47) was prepared according to general procedure B using $d_{2}$-TMDSO in $d_{8}$-toluene. Purification was performed using a gradient of $1 \rightarrow 5 \%$ ethyl acetate in hexanes to afford (47) as a white solid (31 mg, 72\% yield). ${ }^{1} \mathrm{H}$ NMR (400 MHz, $\left.\mathrm{CDCl}_{3}\right): \delta 7.11-7.10(\mathrm{~m}, 4 \mathrm{H})$, 2.46-2.39 (m, 1H), $2.32(\mathrm{~s},<0.08 \mathrm{H})$ 1.90-1.85 (m, $4 \mathrm{H}), 1.49-1.38(\mathrm{~m}, 2 \mathrm{H}), 1.34-1.21(\mathrm{~m}, 7 \mathrm{H}), 1.10-1.00(\mathrm{~m}$, 2H), 0.93-0.89 (m, 3H); $\left.{ }^{13} \mathrm{C} \mathrm{NMR} \mathrm{(100} \mathrm{MHz,} \mathrm{CDCl}_{3}\right): 144.9,135.1,128.9,126.7,44.2,37.3,37.1,34.4,33.7$, $29.2,23.0,20.9,14.1\left(\mathrm{~m}, \mathrm{CD}_{3}\right)$.<smiles>O=C(c1ccccc1)c1ccc2ccccc2c1</smiles>

$\left(d_{3}\right)$-2Methylnapthalene (48) was prepared according to general procedure B using $d_{2}$-TMDSO in $d_{8^{-}}$ toluene. Purification was performed using a gradient of $1 \rightarrow 5 \%$ ethyl acetate in hexanes to afford (48) as a white solid (23 mg, 68\% yield). Characterization data matches the literature for the analogous nondeuterated compound. ${ }^{14}{ }^{1} \mathrm{H}$ NMR $\left(400 \mathrm{MHz}, \mathrm{CDCl}_{3}\right): \delta 7.80-7.78(\mathrm{~d}, J=8.0 \mathrm{~Hz}, 3 \mathrm{H}), 7.79(\mathrm{~m}, 1 \mathrm{H}), 7.45-7.41$ 
(m, 2H), 7.37-7.34 (d, J = 8.4 Hz, 1H), $2.51(\mathrm{~s},<0.07 \mathrm{M}) ;{ }^{13} \mathrm{C}$ NMR $\left(100 \mathrm{MHz}, \mathrm{CDCl}_{3}\right): 135.4,133.6,131.7$, $128.0,127.6,127.5,127.1,126.0,125.7,124.9,21.6\left(m, \mathrm{CD}_{3}\right)$.

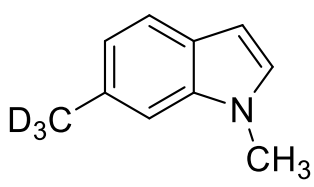

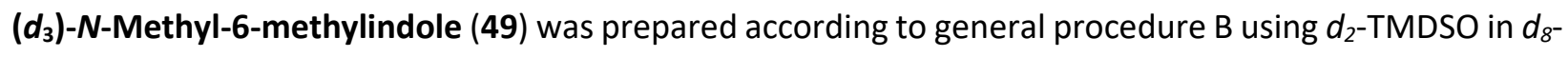
toluene. Purification was performed using a gradient of $1 \rightarrow 5 \%$ ethyl acetate in hexanes to afford (49) as a white solid ( $23 \mathrm{mg}, 65 \%$ yield). Characterization data matches the literature for the analogous nondeuterated compound. ${ }^{30}{ }^{1} \mathrm{H}$ NMR $\left(400 \mathrm{MHz}, \mathrm{CDCl}_{3}\right): \delta 7.55$ (dd, $\left.J=7.8,1.4 \mathrm{~Hz}, 1 \mathrm{H}\right), 7.15(\mathrm{~s}, 1 \mathrm{H}), 7.00-6.98$ $(\mathrm{m}, 2 \mathrm{H}), 6.48-6.46(\mathrm{~m}, 1 \mathrm{H}), 3.77(\mathrm{~s}, 3 \mathrm{H}), 2.53(\mathrm{~s},<0.07 \mathrm{H}) ;{ }^{13} \mathrm{C}$ NMR $\left(100 \mathrm{MHz}, \mathrm{CDCl}_{3}\right):$ 137.0, 131.1, 128.1, $126.2,121.0,120.4,109.1,100.6,32.6,21.8\left(m, C_{3}\right)$. 
6.2. Chemoselectivity between ester reduction and ethereal cleavage

Ester reduction using the $\mathrm{Ni} / \mathrm{TMDSO}_{\mathrm{ICY}} / \mathrm{KO}^{\mathrm{t}} \mathrm{Bu}$ system was done according to a modified general procedure A using $0.20 \mathrm{mmol}$ of starting material, $0.4 \mathrm{~mL}$ of toluene and a reaction time of $12 \mathrm{~h}$, affording products 54-57.

$\underline{\text { General Procedure C (adopted from the method used by Martin and coworkers }{ }^{43} \text { ) }}$

To an oven dried $8 \mathrm{~mL}$ screw-top test-tube, $0.20 \mathrm{mmol}$ of starting material is added alongside an oven dried micro-stir bar. The screw-top test-tube is subsequently brought into a nitrogen-filled glovebox. Once under the inert atmosphere, $0.04 \mathrm{mmol} \mathrm{PCy}_{3}$ and $0.02 \mathrm{mmol} \mathrm{Ni}(\operatorname{cod})_{2}$ are added. $0.4 \mathrm{~mL}$ of toluene is added to the test-tube, followed by $0.20 \mathrm{mmol}$ of 1,1,3,3-tetramethyldisiloxane. The reaction vessel is quickly sealed with a Teflon-septa equipped cap and brought outside of the glovebox, where it is stirred inside of a mineral-oil bath at $600 \mathrm{rpm}$ for 12 hours at $110^{\circ} \mathrm{C}$. After 12 hours, the reaction vessel is allowed to come to room temperature before being opened to the atmosphere. $0.80 \mathrm{mmol}$ of tetra- $\mathrm{n}$ butylammonium fluoride is added slowly as a $1.0 \mathrm{M}$ solution in tetrahydrofuran and the resulting solution is heated to $65^{\circ} \mathrm{C}$ and stirred for 2 hours. The crude reaction solution is subsequently added directly to a $10 \mathrm{~g}$ Biotage SNAP silica-packed column where it is purified on a CombiFlash Rft automated chromatography instrument using a mixture of ethyl acetate in hexanes, affording products 58-60.<smiles>COc1ccccc1-c1ccc(C(C)=O)cn1</smiles>

50

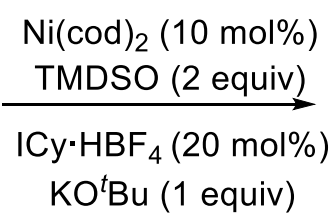

$\mathrm{KO}^{t} \mathrm{Bu}(1$ equiv)

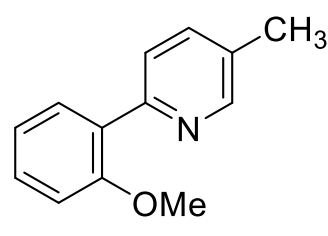

54

2-(2-Methoxyphenyl)-5-methylpyridine (54) was prepared according to a modified general procedure A using $0.2 \mathrm{mmol}$ of starting material, $0.4 \mathrm{~mL}$ of toluene and a reaction time of $12 \mathrm{~h}$. Purification was performed using a gradient of $1 \% \rightarrow 5 \%$ EtOAc in hexane to afford (54) as colorless solid ( $23 \mathrm{mg}, 69 \%$ yield [76\% yield via $\left.\left.{ }^{1} \mathrm{H}-\mathrm{NMR}\right]\right)$. Characterization data matched those previously reported. ${ }^{44}{ }^{1} \mathbf{H}$ NMR $(400 \mathrm{MHz}$, $\left.\mathrm{CDCl}_{3}\right): \delta 8.50(\mathrm{~d}, J=2.4 \mathrm{~Hz}, 1 \mathrm{H}), 7.72-7.66(\mathrm{~m}, 2 \mathrm{H}), 7.49(\mathrm{dd}, J=8.4,2.4 \mathrm{~Hz}, 1 \mathrm{H}), 7.35-7.31(\mathrm{~m}, 1 \mathrm{H}), 7.04$ 
$(\mathrm{t}, J=7.6 \mathrm{~Hz}, 1 \mathrm{H}), 6.97(\mathrm{~d}, J=8.0 \mathrm{~Hz}, 1 \mathrm{H}), 3.83(\mathrm{~s}, 3 \mathrm{H}), 2.34(\mathrm{~s}, 3 \mathrm{H}) ;{ }^{13} \mathrm{C} \mathrm{NMR}\left(100 \mathrm{MHz}, \mathrm{CDCl}_{3}\right): 156.9,153.4$, $149.8,136.3,131.1,131.0,129.6,129.2,124.5,121.0,111.3,55.6,18.2$.

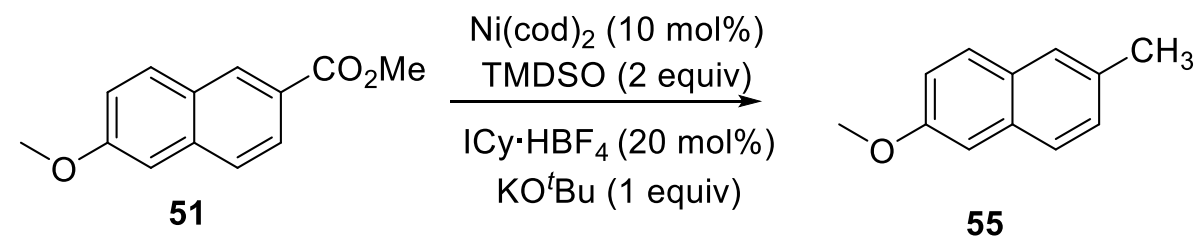

2-Methoxy-6-methylnaphthalene (55) was prepared according to a modified general procedure A using $0.2 \mathrm{mmol}$ of starting material, $0.4 \mathrm{~mL}$ of toluene and a reaction time of $12 \mathrm{~h}$. Purification was done a gradient of $1 \rightarrow 5 \%$ EtOAc in hexanes to afford (55) as off-white solid $\left(27 \mathrm{mg}, 73 \%\right.$ yield [ $88 \%$ yield via ${ }^{1} \mathrm{H}$ NMR]). Characterization data matched those previously reported. ${ }^{45}{ }^{1} \mathbf{H ~ N M R}\left(400 \mathrm{MHz}, \mathrm{CDCl}_{3}\right): \delta 7.64$ (dd, $J=8.4,3.2 \mathrm{~Hz}, 2 \mathrm{H}), 7.54(\mathrm{~s}, 1 \mathrm{H}), 7.28(\mathrm{dd}, J=8.4,2.0 \mathrm{~Hz}, 1 \mathrm{H}), 7.13-7.10(\mathrm{~m}, 2 \mathrm{H}), 3.90(\mathrm{~s}, 3 \mathrm{H}), 2.48(\mathrm{~s}, 3 \mathrm{H})$; ${ }^{13} \mathrm{C}$ NMR $\left(100 \mathrm{MHz}, \mathrm{CDCl}_{3}\right): 157.0,133.0,132.6,129.1,128.7,128.5,126.7,126.5,118.6,105.6,55.2,21.4$.

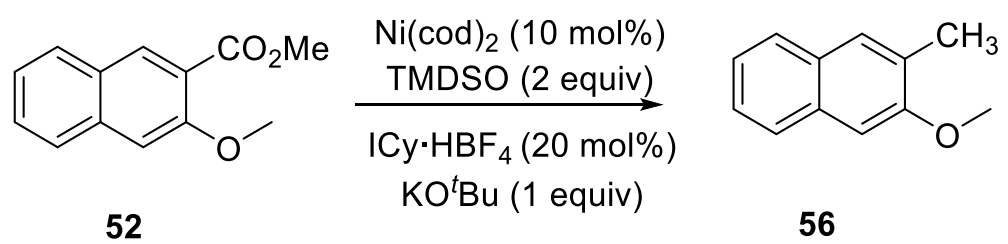

2-Methoxy-3-methylnapthalene (56) was prepared according to a modified general procedure A using $0.2 \mathrm{mmol}$ of starting material, $0.4 \mathrm{~mL}$ of toluene and a reaction time of $12 \mathrm{~h}$. Purification was performed using a gradient of $1 \rightarrow 5 \%$ ethyl acetate in hexanes to afford (56) as an off-white solid (19 $\mathrm{mg}, 63 \%$ yield [78\% yield via $\left.\left.{ }^{1} \mathrm{H}-\mathrm{NMR}\right]\right)$. Characterization data matched those previously reported. ${ }^{46}{ }^{1} \mathbf{H} \mathbf{N M R}(400 \mathrm{MHz}$, $\left.\mathrm{CDCl}_{3}\right): \delta$ 7.73-7.69 (dd, $\left.J=8.2,3.4 \mathrm{~Hz}, 2 \mathrm{H}\right), 7.58(\mathrm{~m}, 1 \mathrm{H}), 7.41-7.33(\mathrm{~m}, 2 \mathrm{H}), 7.10-7.08(\mathrm{~m}, 1 \mathrm{H}) 3.95(\mathrm{~s}, 3 \mathrm{H})$, 2.39 (s, 3H); ${ }^{13} \mathrm{C}$ NMR $\left(100 \mathrm{MHz}, \mathrm{CDCl}_{3}\right): 155.8,131.1,128.7,126.8,126.1,125.3,123.4 .104 .3,55.2,16.8$. 


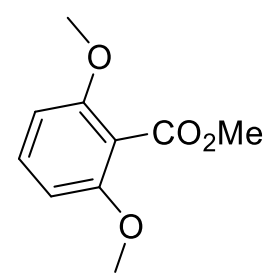

53

$$
\begin{aligned}
& \mathrm{Ni(cod})_{2}(10 \text { mol } \%) \\
& \underset{\mathrm{TMDSO}(2 \text { equiv })}{\longrightarrow} \\
& \underset{\mathrm{ICy} \cdot \mathrm{HBF}_{4}(20 \text { mol } \%)}{\mathrm{KO}{ }^{t} \mathrm{Bu}(1 \text { equiv })}
\end{aligned}
$$

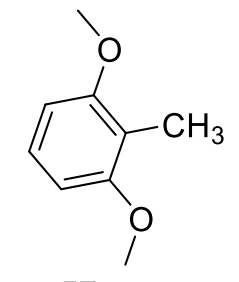

57

1,3-Dimethoxy-2-methylbenzene (57) was prepared according to a modified general procedure A using $0.2 \mathrm{mmol}$ of starting material, $0.4 \mathrm{~mL}$ of toluene and a reaction time of $12 \mathrm{~h}$. Purification was performed using a gradient of $1 \rightarrow 5 \%$ EtOAc in hexanes to afford (57) as colourless solid ( $19 \mathrm{mg}, 62 \%$ yield [ $73 \%$ yield via $\left.\left.{ }^{1} \mathrm{H}-\mathrm{NMR}\right]\right)$. Characterization data matched those previously reported. ${ }^{47}{ }^{1} \mathrm{H}$ NMR $\left(400 \mathrm{MHz}, \mathrm{CDCl}_{3}\right): \delta$ 7.12-7.08 (m, 1H), 6.52 (d, $J=8.4 \mathrm{~Hz}, 2 \mathrm{H}), 3.81(\mathrm{~s}, 6 \mathrm{H}), 2.09(\mathrm{~s}, 3 \mathrm{H}) ;{ }^{13} \mathrm{C}$ NMR $\left(100 \mathrm{MHz}, \mathrm{CDCl}_{3}\right):$ 158.3, $126.1,114.5,103.5,55.7,8.1$.<smiles>COc1ccccc1-c1ccc(C(C)=O)cn1</smiles>

50

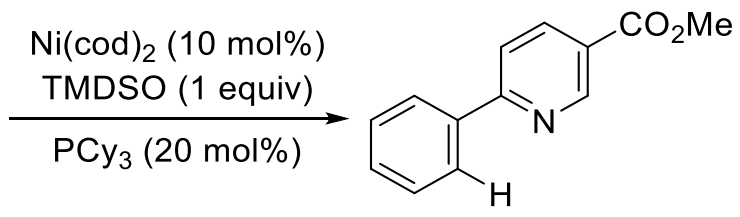

58

Methyl 5-phenylpyridine-3-carboxylate (58) was prepared according to general procedure $C$ to afford (58) $\left(25 \mathrm{mg}, 68 \%\right.$ yield [76\% yield via $\left.{ }^{1} \mathrm{H}-\mathrm{NMR}\right]$ ). Characterization data matched those previously reported..$^{48}{ }^{1} \mathrm{H}$ NMR $\left(400 \mathrm{MHz}, \mathrm{CDCl}_{3}\right): \delta 9.30(\mathrm{~s}, 1 \mathrm{H}), 8.38-8.32(\mathrm{dd}, J=8.4,2.0 \mathrm{~Hz}, 1 \mathrm{H}), 8.08-8.02(\mathrm{dd}, J=$ 9.0, $1.6 \mathrm{~Hz}, 2 \mathrm{H}), 7.83-7.80(\mathrm{~d}, J=8.4 \mathrm{~Hz}, 1 \mathrm{H}), 7.53-7.48(\mathrm{~m}, 3 \mathrm{H}), 3.97(\mathrm{~s}, 3 \mathrm{H}) ;{ }^{13} \mathrm{C} \mathrm{NMR}\left(100 \mathrm{MHz}, \mathrm{CDCl}_{3}\right)$ : 166.0, 160.8, 150.9, 138.1, 137.7, 129.8, 127.9, 124.1, 119.7, 52.3.

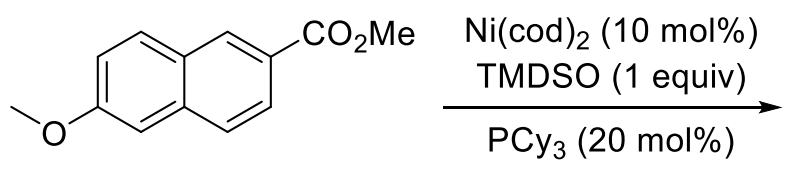

51<smiles>CC(=O)c1ccc2ccccc2c1</smiles>

59

Methyl-2-napthoate (59) was prepared according to general procedure $C$ to afford (59) (15 mg, 57\% yield [60\% yield via $\left.\left.{ }^{1} \mathrm{H}-\mathrm{NMR}\right]\right)$. Characterization data matched those previously reported. ${ }^{43}{ }^{1} \mathrm{H}$ NMR $(400 \mathrm{MHz}$, $\left.\mathrm{CDCl}_{3}\right): \delta 8.62(\mathrm{~s}, 1 \mathrm{H}), 8.08-8.04(\mathrm{dd}, J=8.6,1.6 \mathrm{~Hz}, 1 \mathrm{H}), 7.58-7.53(\mathrm{~m}, 2 \mathrm{H}), 3.98(\mathrm{~s}, 3 \mathrm{H}) ;{ }^{13} \mathrm{C}$ NMR $(100$ $\left.\mathrm{MHz}, \mathrm{CDCl}_{3}\right): 167.1,135.3,132.4,129.2,128.0,127.7,126.3,124.8,51.7$. 


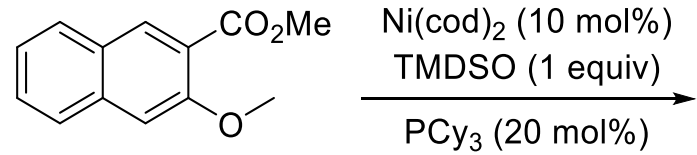

52

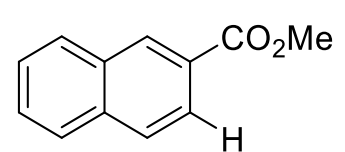

59

Methyl-2-napthoate (59) was prepared according general procedure $C$ to afford (59) (28 $\mathrm{mg}, 79 \%$ yield [90\% yield via $\left.\left.{ }^{1} \mathrm{H}-\mathrm{NMR}\right]\right)$. Characterization data matched those previously reported. ${ }^{43}{ }^{1} \mathrm{H}$ NMR $(400 \mathrm{MHz}$, $\left.\mathrm{CDCl}_{3}\right): \delta 8.62(\mathrm{~s}, 1 \mathrm{H}), 8.08-8.04(\mathrm{dd}, J=8.6,1.6 \mathrm{~Hz}, 1 \mathrm{H}), 7.58-7.53(\mathrm{~m}, 2 \mathrm{H}), 3.98(\mathrm{~s}, 3 \mathrm{H}) ;{ }^{13} \mathrm{C}$ NMR $(100$ $\left.\mathrm{MHz}, \mathrm{CDCl}_{3}\right): 167.1,135.3,132.4,129.2,128.0,127.7,126.3,124.8,51.7$.

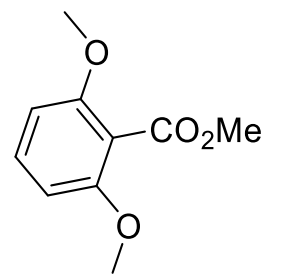

53

$$
\begin{aligned}
& \underset{\mathrm{Ni}(\mathrm{cod})_{2}(10 \mathrm{~mol} \%)}{\mathrm{TMDSO}(1 \text { equiv })} \\
& \underset{\mathrm{PCy}_{3}(20 \mathrm{~mol} \%)}{\longrightarrow}
\end{aligned}
$$

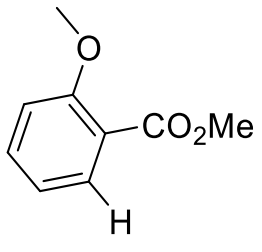

60

Methyl-2-methoxybenzoate (60) was prepared according to general procedure $C$ to afford (60) (21 mg, $68 \%$ yield [78\% yield via $\left.\left.{ }^{1} \mathrm{H}-\mathrm{NMR}\right]\right)$. Characterization data matched those previously reported. ${ }^{49}{ }^{1} \mathbf{H} \mathbf{~ N M R}$ (400 MHz, $\left.\mathrm{CDCl}_{3}\right): \delta$ 7.74-7.69 (dd, $\left.J=7.5,1.5 \mathrm{~Hz}, 1 \mathrm{H}\right), 7.44-7.38(\mathrm{~m}, 1 \mathrm{H}), 6.97-6.93(\mathrm{~m}, 2 \mathrm{H}), 3.87(\mathrm{~s}, 3 \mathrm{H})$, 3.85 (s, 3H); ${ }^{13} \mathrm{C}$ NMR (100 MHz, $\left.\mathrm{CDCl}_{3}\right): 164.7,158.6,133.1,130.8,118.9,117.6,111.3,54.7,51.4$. 
6.3. Kinetic experiments

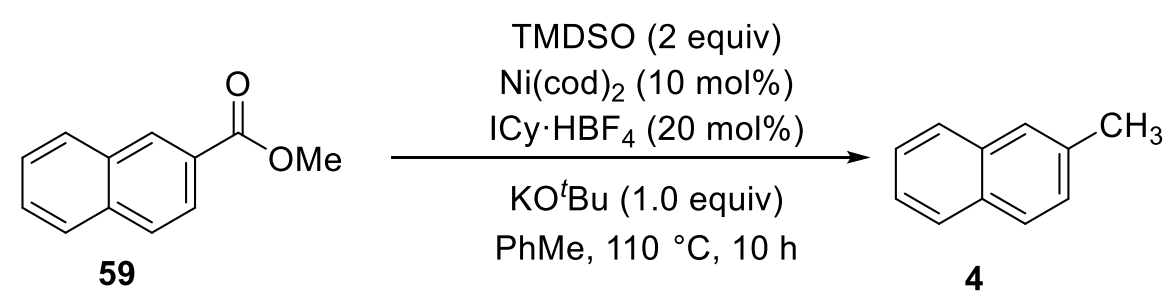

All individual kinetics experiments were performed according to general procedure A. Ten reactions were set up in parallel and stopped after $10 \mathrm{~min}, 20 \mathrm{~min}, 30 \mathrm{~min}, 1 \mathrm{~h}, 2 \mathrm{~h}, 3 \mathrm{~h}, 5 \mathrm{~h}, 7 \mathrm{~h}, 9 \mathrm{~h}$ and $10 \mathrm{~h}$. Yields were obtained via ${ }^{1} \mathrm{H}-\mathrm{NMR}$ using 1,3,5-trimethoxybenzene as internal standard. Variable time normalization analysis was conducted on the obtained time vs. yield data based on the method described by Bures and co-workers ${ }^{51}$ in order to determine the observed rate equation.

Standard reaction conditions were 0.25 M 2-methyl naphthoate (59), 0.25 M KOBu, 0.50 M TMDSO, 0.025 $\mathrm{M} \mathrm{Ni}(\operatorname{cod})_{2}$ and $0.05 \mathrm{M} \mathrm{ICy} \cdot \mathrm{HBF}_{4}$. Scheme $\mathbf{S 7}$ was generated by setting [catalyst] $\left(\mathrm{Ni}(\mathrm{cod})_{2}+\mathrm{ICy} \cdot \mathrm{HBF}_{4}, 1: 2\right.$ ratio) to $0.025 \mathrm{M}$ (standard conditions), $0.050 \mathrm{M}$ and $0.075 \mathrm{M}$. Scheme S8 was generated by varying [TMDSO], setting it to $0.50 \mathrm{M}$ (standard conditions), $0.75 \mathrm{M}$, and $1.0 \mathrm{M}$. Scheme $\mathbf{S 9}$ was generated by varying [59], setting it to $0.25 \mathrm{M}$ (standard conditions), $0.5 \mathrm{M}$ and $0.75 \mathrm{M}$. Scheme S10 was generated by varying $\left[\mathrm{KO}^{t} \mathrm{Bu}\right.$ ], setting it to $0.025 \mathrm{M}$ (standard conditions), $0.038 \mathrm{M}$ and $0.050 \mathrm{M}$.

Results of these VTNA experiments indicate a positive order for all tested components, suggesting that each component plays a role in the rate-determining step. While good overlay was observed when normalizing the $\mathrm{X}$ axis assuming first order behavior, the current data cannot eliminate fractional orders between 0.5 to 1.5 . 

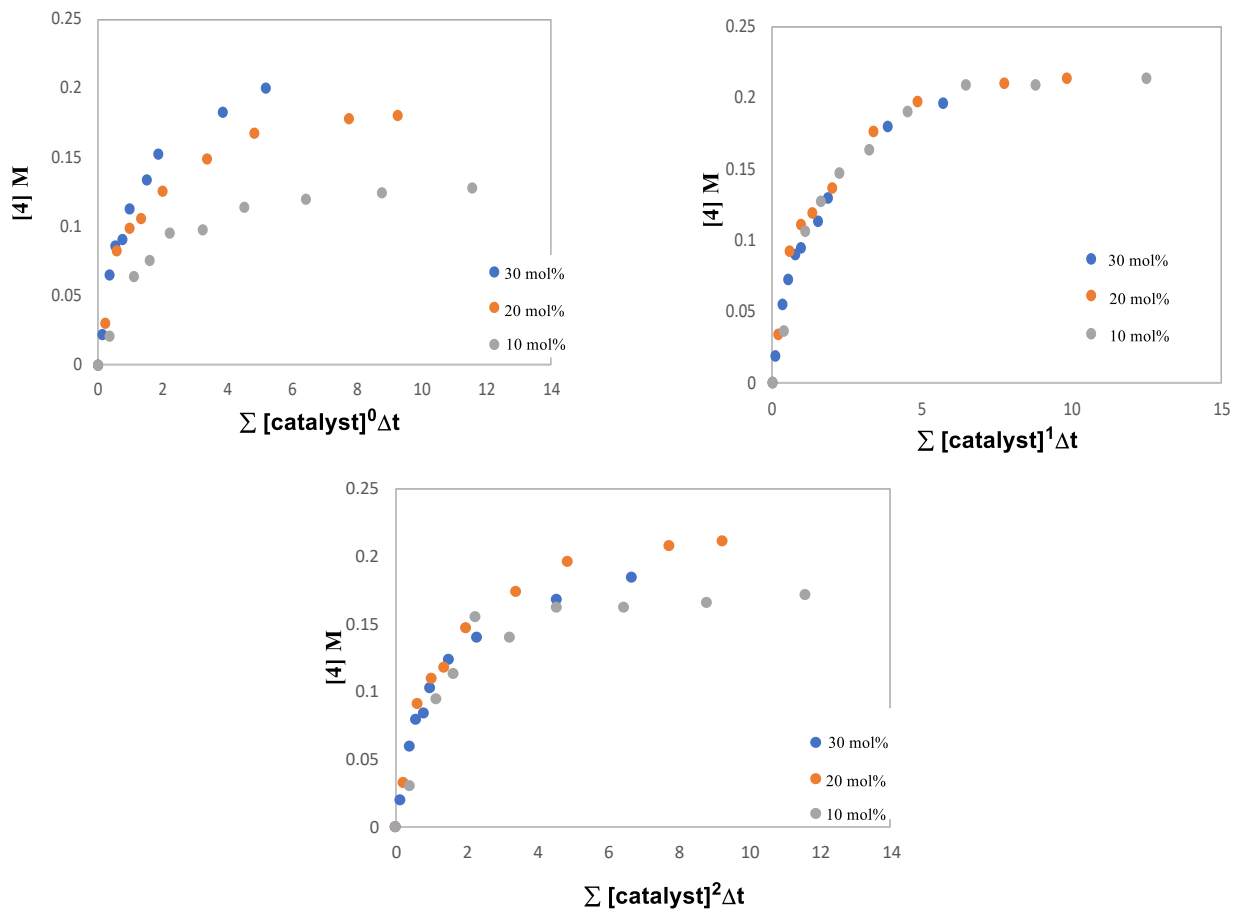

Scheme S7. Variation in catalyst: Variable time normalization plots illustrates positive order dependency.
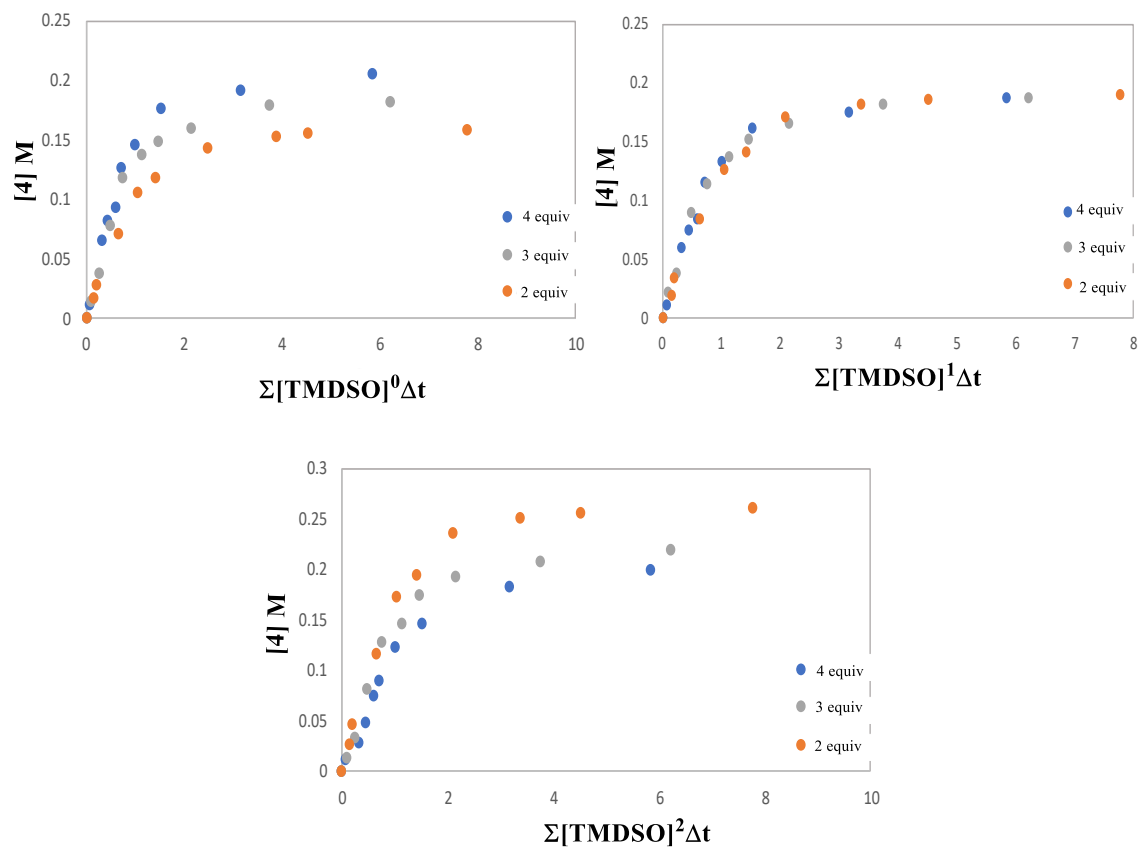

Scheme S8. Variation in TMDSO: Variable time normalization plots illustrates positive order dependency. 

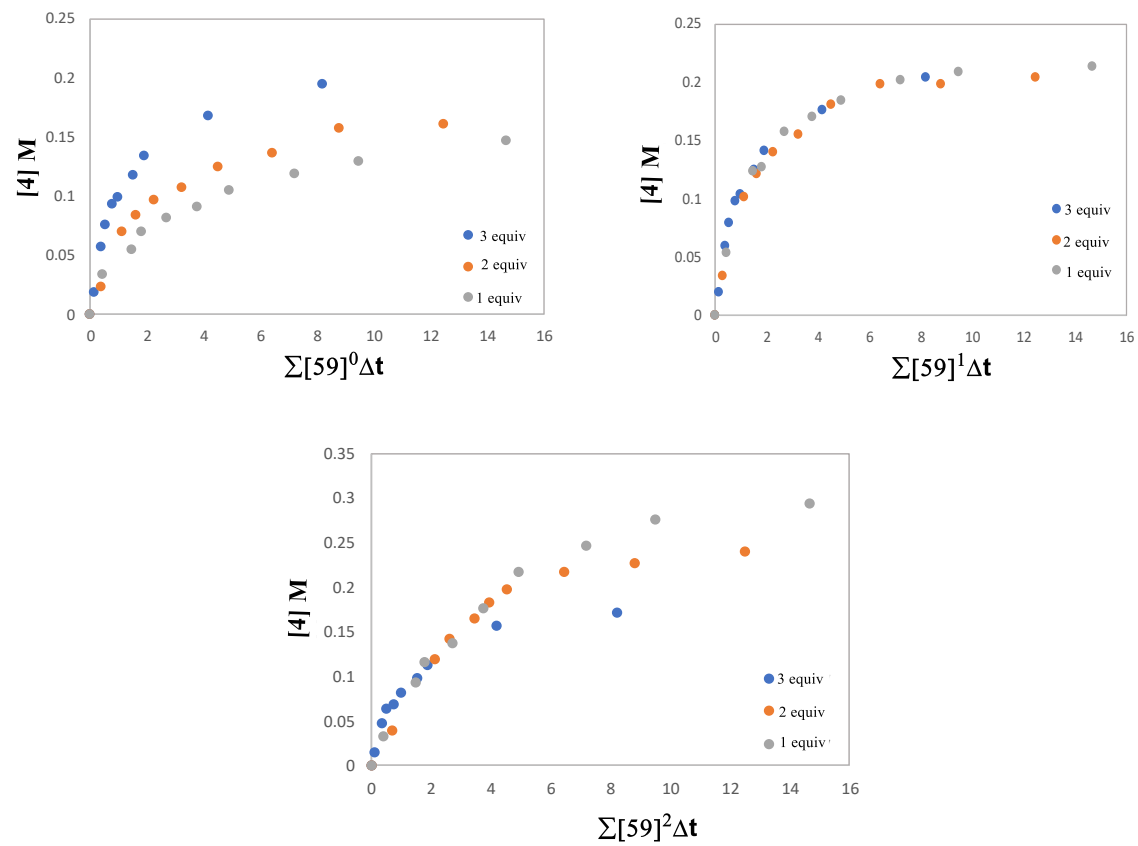

Scheme S9. Variation in [59]: Variable time normalization plots illustrates positive order dependency.
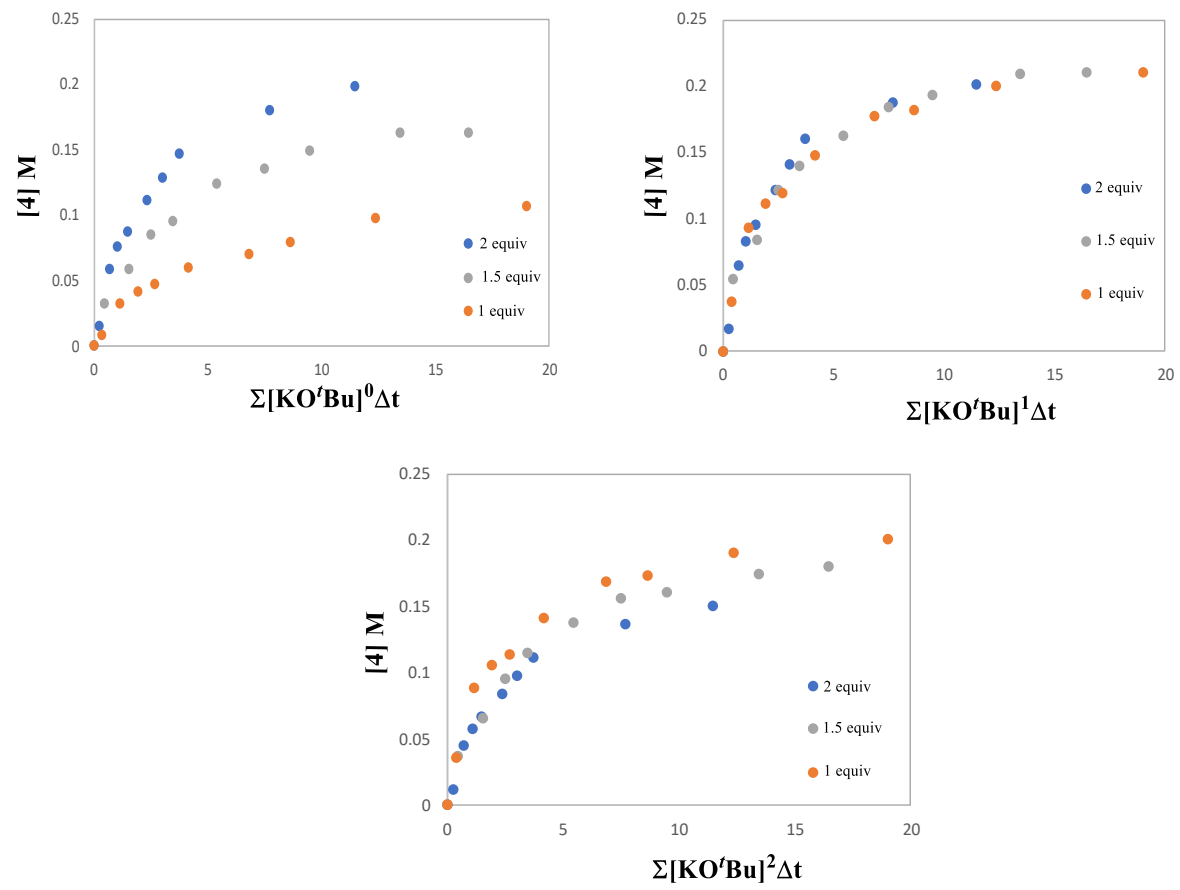

Scheme S10. Variation in $\mathrm{KO}^{\mathrm{t}} \mathrm{Bu}$ : Variable time normalization plots illustrates positive order dependency. 


\subsection{Reduction of proposed intermediates}

To probe the catalytic reaction of benzyl alcohols, various indole-bearing alcohol derivatives were subjected to the reducing conditions to determine if this step of the reaction behaved differently in isolation (Table S8). Using 1.0 equivalents of TMDSO provided indole $\mathbf{2}$ in $58 \%$ yield (entry 1 ). Use of 0.6 equivalents TMDSO (formally 1.2 equivalents of hydride) gave a slightly lower yield (entry 2). As was the case in the direct reduction starting from the ester, both $\mathrm{Ni}$ and $\mathrm{KO}^{t} \mathrm{Bu}$ were required for conversion (entries 3,4 ). Removing the base from the reaction mixture failed to afford product (entry 5), however base was only required in catalytic quantity to afford product (entry 6), contrasting the results when the ester was used as starting material. Replacing $\mathrm{KO}^{t} \mathrm{Bu}$ with KF failed to afford product (entry 7), presumably because KF is insufficiently basic to deprotonate the NHC ligand. Adding 20 mol\% of KO ${ }^{t} \mathrm{Bu}$ restored reactivity (entry 8 ), as did substituting $\mathrm{KOH}$ as the base (entry 9). Reactions performed using TBS, TMS, Me and Ph protected alcohols led to a higher yield than that which was obtained when using the unprotected analog (entries 10-19). These species are more analogous to the mixture of silyl-alcohol species that are believed to form in situ via ester hydrosilylation. Finally, the corresponding aldehyde underwent reduction upon exposure to our reaction conditions, confirming it as a viable intermediate in this reaction (entry 20).

Table S8. Reduction of proposed intermediates

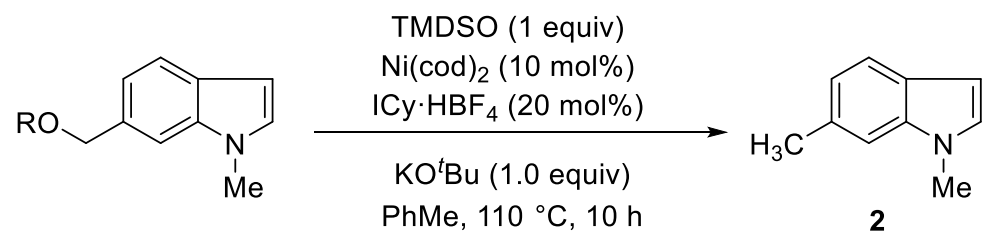

\begin{tabular}{lccc}
\hline entry & $\mathbf{R}$ & deviation from standard conditions & yield, $\mathbf{2}$ [\%] \\
\hline $\mathbf{1}$ & $\mathrm{R}=\mathrm{H}$ & none & 53 \\
$\mathbf{2}$ & $\mathrm{R}=\mathrm{H}$ & 0.6 equiv TMDSO & 41 \\
$\mathbf{3}$ & $\mathrm{R}=\mathrm{H}$ & 0.3 equiv TMDSO & 18 \\
$\mathbf{4}$ & $\mathrm{R}=\mathrm{H}$ & No Ni(cod) 2 & 0 \\
$\mathbf{5}$ & $\mathrm{R}=\mathrm{H}$ & $\mathrm{ICy}($ free $)$, no base & 0 \\
$\mathbf{6}$ & $\mathrm{R}=\mathrm{H}$ & 20 mol\% KOtBu & 24 \\
$\mathbf{7}$ & $\mathrm{R}=\mathrm{H}$ & $\mathrm{KF}\left(1.0\right.$ equiv) instead of $K \mathrm{O}^{t} \mathrm{Bu}$ & trace
\end{tabular}




\begin{tabular}{|c|c|c|c|}
\hline 8 & $\mathrm{R}=\mathrm{H}$ & $\mathrm{KF}(1.0$ equiv $)+20 \mathrm{~mol} \% \mathrm{KO}^{t} \mathrm{Bu}$ & 47 \\
\hline 9 & $\mathrm{R}=\mathrm{H}$ & $\mathrm{KOH}$ (1.0 equiv) & 31 \\
\hline 10 & $\mathrm{R}=\mathrm{TBS}$ & $\mathrm{KF}$ (1.0 equiv) instead of $\mathrm{KO}^{t} \mathrm{Bu}$ & trace \\
\hline 11 & $\mathrm{R}=\mathrm{TBS}$ & $\mathrm{KF}(1.0$ equiv $)+20 \mathrm{~mol} \% \mathrm{KO}^{t} \mathrm{Bu}$ & 74 \\
\hline 12 & $\mathrm{R}=\mathrm{TBS}$ & none & 74 \\
\hline 13 & $\mathrm{R}=\mathrm{TBS}$ & 0.6 equiv TMDSO & 63 \\
\hline 14 & $\mathrm{R}=\mathrm{TBS}$ & $\mathrm{No} \mathrm{Ni}(\operatorname{cod})_{2}$ & 0 \\
\hline 15 & $\mathrm{R}=\mathrm{TBS}$ & ICy (free), no base & 0 \\
\hline 16 & $\mathrm{R}=\mathrm{TMS}$ & none & 71 \\
\hline 17 & $\mathrm{R}=\mathrm{Me}$ & none & 77 \\
\hline 18 & $\mathrm{R}=\mathrm{Me}$ & $\mathrm{KF}(1.0 \mathrm{eq})+20 \mathrm{~mol} \% \mathrm{KOtBu}$ & 70 \\
\hline 19 & $\mathrm{R}=\mathrm{Ph}$ & none & 51 \\
\hline 20 & - & aldehyde as starting material & 73 \\
\hline
\end{tabular}


Kinetic studies were performed in order to learn more about the fate of the methyl ester as it passes through the alcohol intermediate. Ester to alkane reduction was performed using general procedure $\mathrm{A}$ with methyl 2-naphthoate. Alcohol to alkane reduction was performed using general procedure A with napthalene-2-methanol as starting material. Ester to alcohol reduction was performed using a modified general procedure $A$, in which the catalyst and ligand were removed. The kinetic plots indicate that ester to alcohol reduction is exceptionally rapid relative to the subsequent steps.
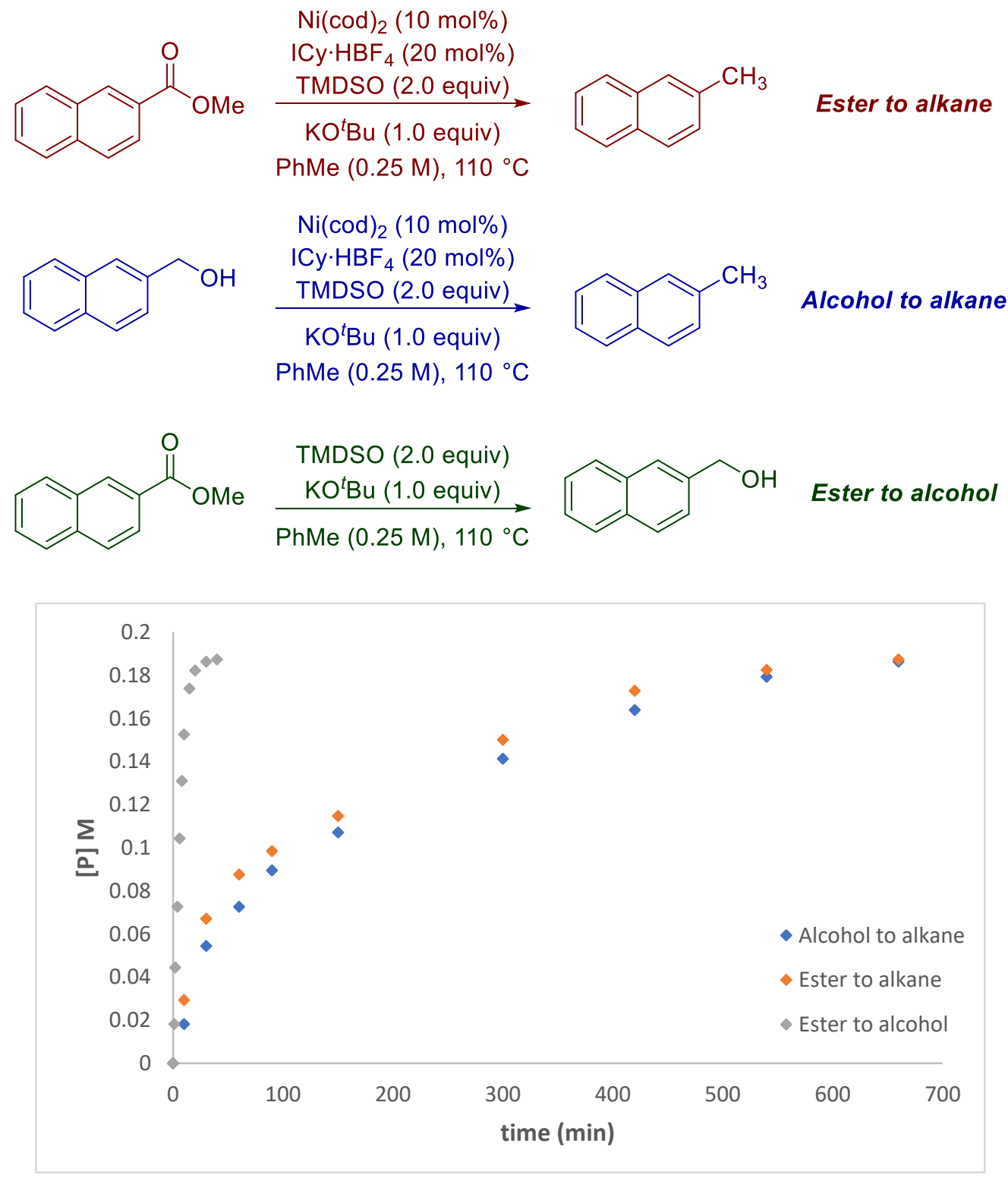

Scheme S11. Product vs. time curves for the reduction of methyl-2-napthoate and napthalene-2-methanol 


\subsection{Reduction of other alkyl and aryl esters}

The following esters were reduced according to general procedure A, confirming that the reaction is not limited to just the reduction of methyl esters (Scheme S10). Both ester-bearing naphthalene species (S14S16) and ester-bearing indole species (S17-S19) were tested.

Other successfully reduced esters<smiles>O=C(OCc1ccccc1)c1ccc2ccccc2c1</smiles>

S14. $66 \%$<smiles>Cn1ccc2ccc(C(=O)OCc3ccccc3)cc21</smiles>

S17. $75 \%$<smiles>CC(C)OC(=O)c1ccc2ccccc2c1</smiles>

S15. $72 \%$

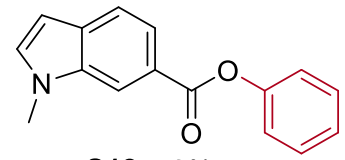

S18. $73 \%$

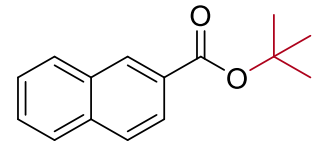

S16. $76 \%$

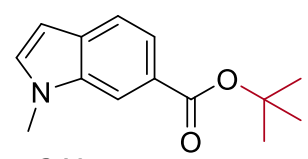

S19. $77 \%$

Scheme S12. Depicting the types of esters that could be reduced according to general procedure A

To further illustrate the selectivity of our method, we prepared S20 from the corresponding arenol. Subjecting this compound to our reaction conditions led to selective reduction of the benzyl methyl ether in the presence of an aryl silyl ether.

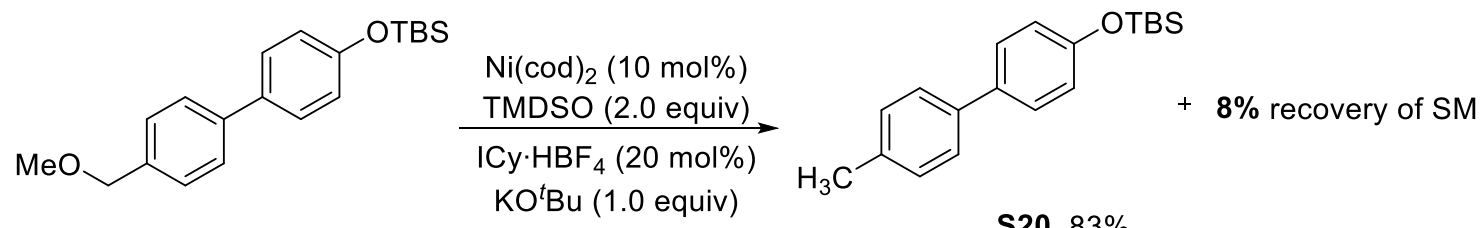

4-[[(1,1-Dimethylethyl)dimethylsilyl]oxy]-4'-methyl-1,1'-biphenyl (S20) was prepared according to general procedure A. Purification was performed using a gradient of $10 \rightarrow 50 \%$ ethyl acetate in hexanes to afford (S20) (29 mg, 83\% yield). Characterization data matched those previously reported. ${ }^{50}{ }^{\mathbf{1}} \mathbf{H} \mathbf{N M R}$ (400 MHz, $\left.\mathrm{CDCl}_{3}\right): \delta$ 7.60-7.58 (m, 2H), 7.51-7.47 (m, 2H), 7.45-7.41 (m, 2H), 7.35-7.31 (m, 1H), 7.26-7.25 (m, 1H), $2.41(\mathrm{~s}, 3 \mathrm{H}), 0.99(\mathrm{~s}, 9 \mathrm{H}), 0.38(\mathrm{~s}, 6 \mathrm{H}) ;{ }^{13} \mathrm{C}$ NMR (100 MHz, $\left.\mathrm{CDCl}_{3}\right)$ : 141.1, 138.3, 137.0, 129.5, 128.7, 128.6, 127.0, 126.9, 25.3, 21.1, 19.1, 1.6. Accurate mass (EI): Theoretical: 298.1804. Found: 298.1811. Spectral Accuracy: 96.8\%. 


\subsection{Kinetic isotope experiments}

In order to determine the kinetic isotope effects of the reduction reaction, two parallel reductions of napthalene-2-methanol were set up - one with commercial TMDSO and one with $d_{2}$-TMDSO. Aliquots of each of these reactions were taken after $5,10,15,30$ and 60 minutes and subsequently analyzed by GCFID. Product formation vs. time data was plotted and the initial rates were measured to determine a kinetic isotope effect of 2.24 .
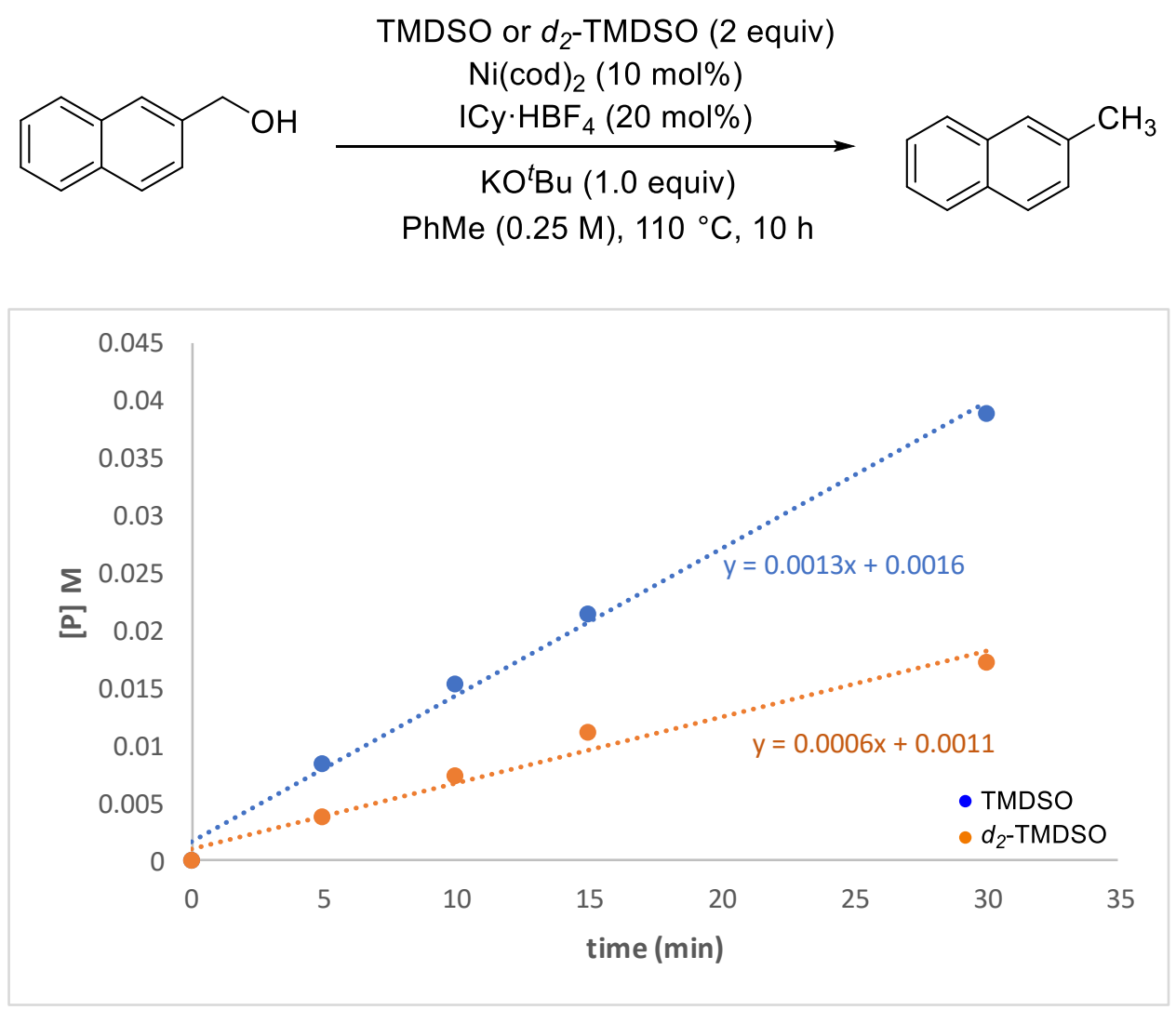

Scheme S13. Initial reaction progress in the presence of TMDSO or $d_{2}$-TMDSO 


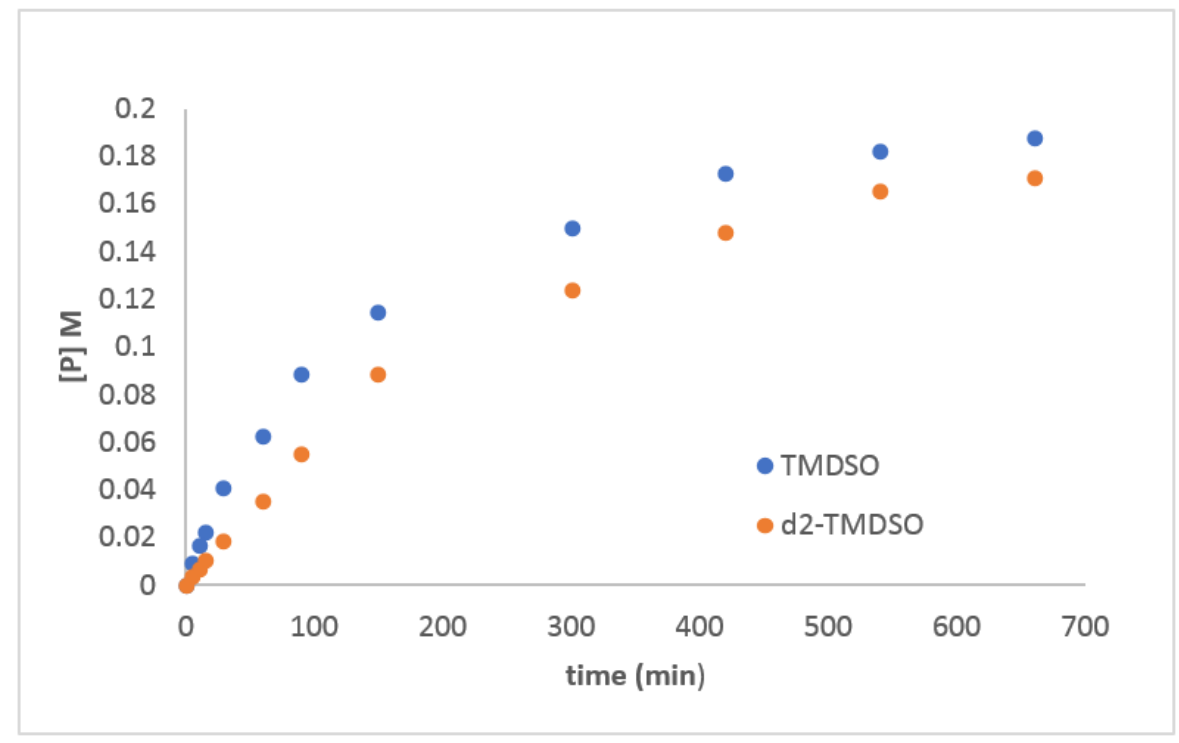

Scheme S14. Total reaction progress in the presence of TMDSO or $d_{2}$-TMDSO

Comparable results were obtained by intermolecular competition kinetic-isotope experiments performed using a 50:50 ratio of TMDSO to $d_{2}$-TMDSO. These experiments resulted in $27 \%$ deuterium incorporation in the methyl reduction product, providing further evidence for a primary-kinetic isotope effect.

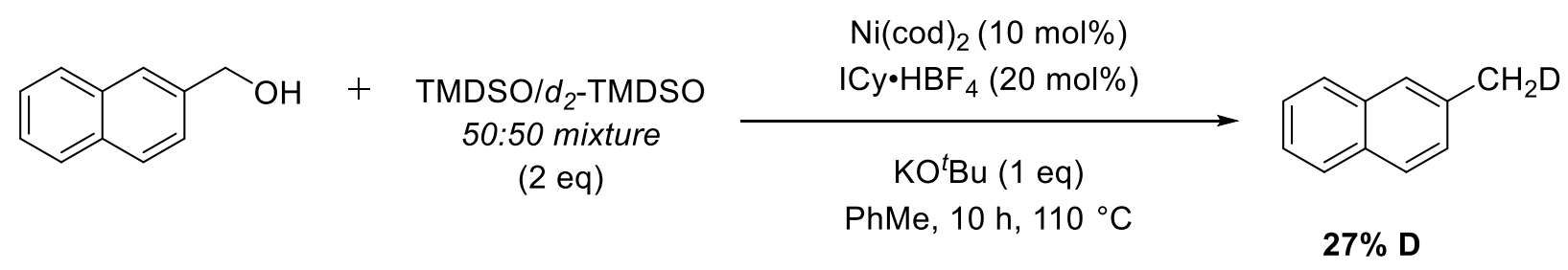




\subsection{Solubility of $K \mathrm{KO}^{t} \mathrm{Bu}$}

In the kinetic experiments detailed in Scheme S10, the amount of TMDSO was fixed at 2.0 equiv, while $\mathrm{KO}^{t} \mathrm{Bu}$ varied from 1.0 to 2.0 equiv. Experiments with larger amounts of $\mathrm{KO} \mathrm{B}^{\mathrm{B}} \mathrm{B}$ showed a positive effect on the rate, but did not overlay with the kinetic plots normalized for first order behavior. Given the anticipated low solubility of $\mathrm{KO}^{t} \mathrm{Bu}$ in toluene, we carried tested the homogeneity of different mixtures of $\mathrm{KO}^{t} \mathrm{Bu}$ and TMDSO (Scheme S15). It was observed that TMDSO was able to solubilize $\mathrm{KO}^{t} \mathrm{Bu}$ entirely, provided that it is TMDSO is in in equal-or-greater stoichiometric amounts relative to the $\mathrm{KO}^{t} \mathrm{Bu}$. Above that point, a slurry was observed. This suggests that the reaction is indeed positive order in $\mathrm{KO}^{\mathrm{t}} \mathrm{Bu}$, but becomes heterogeneous and thus less kinetically tractable at high concentrations of $\mathrm{KO}^{t} \mathrm{Bu}$.

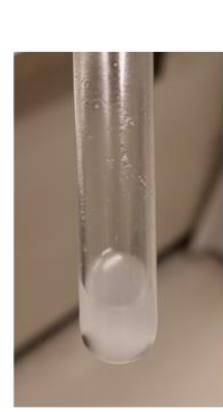

$\mathrm{PhMe}+\mathrm{KO}^{\mathrm{t} B u}(1 \mathrm{eq})$
$\mathrm{PhMe}+\mathrm{KO}^{\mathrm{t} B u}(1 \mathrm{eq})$

$+\operatorname{TMDSO}(2 \mathrm{eq})$

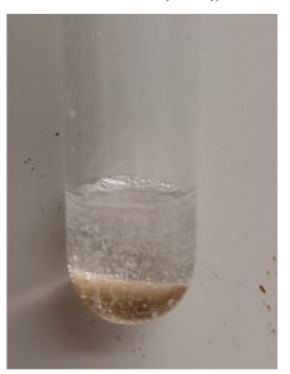

$\mathrm{PhMe}+\mathrm{KO}^{t} \mathrm{Bu}(1 \mathrm{eq})(L)$
$\mathrm{PhMe}+\mathrm{KO} O^{t} \mathrm{Bu}(1 \mathrm{eq})$ $+\operatorname{TMDSO}(2 \mathrm{eq})(\boldsymbol{R})$
$\mathrm{PhMe}+\mathrm{KO} \mathrm{t}^{\mathrm{Bu}}(1 \mathrm{eq})+\mathrm{TMDSO}(1 \mathrm{eq})(L)$ $\mathrm{PhMe}+\mathrm{KO}{ }^{t} \mathrm{Bu}(2 \mathrm{eq})+$ TMDSO (2 eq) $(\boldsymbol{R})$

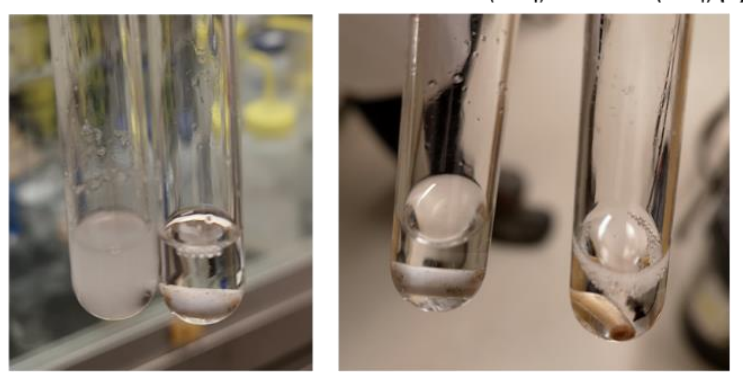

$\mathrm{PhMe}+\mathrm{KO}^{t} \mathrm{Bu}(3 \mathrm{eq})+\mathrm{TMDSO}(2 \mathrm{eq})(L)$ $\mathrm{PhMe}+\mathrm{KO}^{t} \mathrm{Bu}(3 \mathrm{eq})+\mathrm{TMDSO}(3 \mathrm{eq})(\boldsymbol{R})$

Scheme S15. Solubility of $K O^{t} \mathrm{Bu}$ in toluene upon mixing with TMDSO 


\section{Appendix}

Data associated with the creation of the VTNA plots

Determining the order with respect to catalyst

First order:

\begin{tabular}{|c|c|c|c|c|c|c|}
\hline time & tCAT^1 & [product] $\mathrm{mM}$ & tCAT^1 & [product] $\mathrm{mM}$ & tCAT^1 & [product] $\mathrm{mM}$ \\
\hline $0 m$ & 0.0000 & 0.0000 & 0.0000 & 0.0000 & 0.0000 & 0.0000 \\
\hline $10 \mathrm{~m}$ & 0.1193 & 0.0181 & 0.2126 & 0.0332 & 0.3738 & 0.0355 \\
\hline $30 \mathrm{~m}$ & 0.3580 & 0.0543 & 0.5927 & 0.0923 & 1.1215 & 0.1065 \\
\hline $60 \mathrm{~m}$ & 0.5374 & 0.0726 & 0.9769 & 0.1104 & 1.6124 & 0.1273 \\
\hline $90 \mathrm{~m}$ & 0.7558 & 0.0894 & 1.3425 & 0.1185 & 2.2203 & 0.1474 \\
\hline $2.5 \mathrm{~h}$ & 0.9694 & 0.0947 & 1.9901 & 0.1367 & 3.2260 & 0.1627 \\
\hline $5 h$ & 1.5078 & 0.1132 & 3.3833 & 0.1755 & 4.5235 & 0.1901 \\
\hline 7h & 1.8736 & 0.1288 & 4.8469 & 0.1974 & 6.4453 & 0.2083 \\
\hline $9 \mathrm{~h}$ & 3.8635 & 0.1792 & 7.7435 & 0.2101 & 8.7950 & 0.2084 \\
\hline $11 \mathrm{~h}$ & 5.7179 & 0.1963 & 9.8457 & 0.2136 & 12.4875 & 0.2140 \\
\hline
\end{tabular}

Zeroth order:

\begin{tabular}{|ccccccc|}
\hline time & tCAT^0 & [product] $\mathbf{~ m M ~}$ & tCAT^0 & [product] mM & tCAT^0 & [product] mM \\
$\mathbf{1 0 m}$ & 0.119 & 0.021 & 0.213 & 0.030 & 0.374 & 0.0212 \\
$\mathbf{3 0 m}$ & 0.358 & 0.064 & 0.593 & 0.083 & 1.121 & 0.0636 \\
$\mathbf{6 0 m}$ & 0.537 & 0.086 & 0.977 & 0.099 & 1.612 & 0.0761 \\
$\mathbf{9 0 m}$ & 0.756 & 0.091 & 1.342 & 0.106 & 2.220 & 0.0951 \\
$\mathbf{2 . 5 h}$ & 0.969 & 0.112 & 1.990 & 0.125 & 3.226 & 0.0972 \\
$\mathbf{5 h}$ & 1.508 & 0.134 & 3.383 & 0.149 & 4.523 & 0.1135 \\
$\mathbf{7 h}$ & 1.874 & 0.153 & 4.847 & 0.167 & 6.445 & 0.1194 \\
$\mathbf{9 h}$ & 3.863 & 0.183 & 7.743 & 0.178 & 8.795 & 0.1245 \\
$\mathbf{1 1 h}$ & 5.179 & 0.200 & 9.246 & 0.181 & 11.588 & 0.1278 \\
\hline
\end{tabular}

Second order:

\begin{tabular}{|rcccccc|}
\hline time & tCAT^2 & [product] $\mathbf{~ m M}$ & tCAT^2 & [product] $\mathbf{~ m M}$ & tCAT^2 & [product] mM \\
$\mathbf{0 m}$ & 0.000 & 0.000 & 0.000 & 0.000 & 0.000 & 0.000 \\
$\mathbf{1 0 m}$ & 0.119 & 0.020 & 0.213 & 0.033 & 0.374 & 0.030 \\
$\mathbf{3 0 m}$ & 0.358 & 0.059 & 0.593 & 0.092 & 1.121 & 0.095 \\
$\mathbf{6 0 m}$ & 0.537 & 0.079 & 0.977 & 0.110 & 1.612 & 0.114 \\
$\mathbf{9 0 m}$ & 0.756 & 0.084 & 1.342 & 0.118 & 2.220 & 0.156 \\
$\mathbf{2 . 5 h}$ & 0.969 & 0.103 & 1.990 & 0.147 & 3.226 & 0.141 \\
$\mathbf{5 h}$ & 1.508 & 0.124 & 3.383 & 0.174 & 4.523 & 0.162 \\
$\mathbf{7 h}$ & 2.274 & 0.141 & 4.847 & 0.196 & 6.445 & 0.162 \\
$\mathbf{9 h}$ & 4.563 & 0.168 & 7.743 & 0.209 & 8.795 & 0.166 \\
$\mathbf{1 1 h}$ & 6.679 & 0.184 & 9.246 & 0.212 & 11.588 & 0.172 \\
\hline
\end{tabular}


Determining the order with respect to $K O^{t} B u$

First order:

\begin{tabular}{|c|c|c|c|c|c|c|}
\hline time & tCAT^1 & [product] $\mathrm{mM}$ & tCAT^1 & [product] $\mathrm{mM}$ & tCAT^1 & [product] $\mathrm{mM}$ \\
\hline $0 m$ & 0.000 & 0.0000 & 0.000 & 0.000 & 0.000 & 0.000 \\
\hline $10 \mathrm{~m}$ & 0.255 & 0.0168 & 0.377 & 0.0371 & 0.490 & 0.0548 \\
\hline $30 \mathrm{~m}$ & 0.716 & 0.0645 & 1.185 & 0.0927 & 1.541 & 0.0841 \\
\hline $60 \mathrm{~m}$ & 1.075 & 0.0834 & 1.954 & 0.1109 & 2.540 & 0.1221 \\
\hline $90 \mathrm{~m}$ & 1.512 & 0.0960 & 2.685 & 0.1190 & 3.490 & 0.1396 \\
\hline $2.5 \mathrm{~h}$ & 2.376 & 0.1217 & 4.179 & 0.1485 & 5.433 & 0.1623 \\
\hline $5 h$ & 3.016 & 0.1415 & 6.845 & 0.1781 & 7.490 & 0.1844 \\
\hline $7 \mathrm{~h}$ & 3.747 & 0.1609 & 8.639 & 0.1820 & 9.478 & 0.1937 \\
\hline $9 h$ & 7.727 & 0.1876 & 12.395 & 0.2006 & 13.490 & 0.2095 \\
\hline $11 \mathrm{~h}$ & 11.484 & 0.2014 & 19.035 & 0.2111 & 16.490 & 0.2104 \\
\hline
\end{tabular}

Zeroth order:

\begin{tabular}{|rcccccc|}
\hline time & tCAT^0 $^{\mathbf{0}}$ & [product] $\mathbf{~ m M}$ & tCAT^0 $^{\wedge}$ & [product] $\mathbf{~ m M}$ & tCAT^0 & [product] mM \\
$\mathbf{0 m}$ & 0.0000 & 0.0000 & 0.0000 & 0.0000 & 0.0000 & 0.0000 \\
$\mathbf{1 0 m}$ & 0.2547 & 0.0153 & 0.4902 & 0.0324 & 0.3770 & 0.0082 \\
$\mathbf{3 0 m}$ & 0.7159 & 0.0586 & 1.541 & 0.0578 & 1.185 & 0.0316 \\
$\mathbf{6 0 m}$ & 1.075 & 0.0757 & 2.540 & 0.0848 & 1.954 & 0.0408 \\
$\mathbf{9 0 m}$ & 1.512 & 0.0872 & 3.490 & 0.0947 & 2.685 & 0.0470 \\
$\mathbf{2 . 5 h}$ & 2.376 & 0.1105 & 5.433 & 0.1240 & 4.179 & 0.0596 \\
$\mathbf{5 h}$ & 3.016 & 0.1285 & 7.490 & 0.1348 & 6.845 & 0.0693 \\
$\mathbf{7 h}$ & 3.747 & 0.1462 & 9.478 & 0.1487 & 8.639 & 0.0788 \\
$\mathbf{9 h}$ & 7.727 & 0.1795 & 13.490 & 0.1624 & 12.395 & 0.0967 \\
$\mathbf{1 1 h}$ & 11.484 & 0.1979 & 16.490 & 0.1631 & 19.035 & 0.1067 \\
\hline
\end{tabular}

Second order:

\begin{tabular}{|c|c|c|c|c|c|c|}
\hline time & tCAT^2 & [product] $\mathrm{mM}$ & tCAT^2 & [product] mM & tCAT^2 & [product] $\mathrm{mM}$ \\
\hline Om & 0 & 0.0000 & 0 & 0 & 0 & 0 \\
\hline $10 \mathrm{~m}$ & 0.255 & 0.0116 & 0.490 & 0.03654 & 0.3770 & 0.0352 \\
\hline $30 \mathrm{~m}$ & 0.716 & 0.0445 & 1.541 & 0.0648 & 1.185 & 0.0880 \\
\hline $60 \mathrm{~m}$ & 1.075 & 0.0575 & 2.540 & 0.09467 & 1.954 & 0.1052 \\
\hline $90 \mathrm{~m}$ & 1.512 & 0.0663 & 3.490 & 0.11474 & 2.685 & 0.1129 \\
\hline $2.5 \mathrm{~h}$ & 2.376 & 0.0840 & 5.433 & 0.1374 & 4.179 & 0.1409 \\
\hline $5 h$ & 3.016 & 0.0976 & 7.490 & 0.1564 & 6.845 & 0.1690 \\
\hline $7 \mathrm{~h}$ & 3.747 & 0.1110 & 9.478 & 0.161 & 8.639 & 0.1726 \\
\hline $9 \mathrm{~h}$ & 7.727 & 0.1363 & 13.490 & 0.1746 & 12.395 & 0.1903 \\
\hline $11 \mathrm{~h}$ & 11.484 & 0.1503 & 16.490 & 0.1796 & 19.035 & 0.2003 \\
\hline
\end{tabular}


Determining the order with respect to TMDSO

First order:

\begin{tabular}{|rcccccc|}
\hline time & tCAT^1 & [product] $\mathbf{~ m M}$ & tCAT^1 & [product] mM & tCAT^1 & [product] mM \\
$\mathbf{0 m}$ & 0 & 0 & 0 & 0 & 0 & 0 \\
$\mathbf{1 0 m}$ & 0.0675 & 0.0108 & 0.0847 & 0.02160 & 0.14582 & 0.01960 \\
$\mathbf{3 0 m}$ & 0.3129 & 0.0592 & 0.2485 & 0.03749 & 0.203492 & 0.03384 \\
$\mathbf{6 0 m}$ & 0.4380 & 0.0751 & 0.4856 & 0.08919 & 0.6427052 & 0.08468 \\
$\mathbf{9 0 m}$ & 0.5901 & 0.0847 & 0.7479 & 0.1138 & 1.042 & 0.1263 \\
$\mathbf{2 . 5 h}$ & 0.7092 & 0.1149 & 1.140 & 0.1377 & 1.412 & 0.1415 \\
$\mathbf{5 h}$ & 1.002 & 0.1330 & 1.469 & 0.1520 & 2.090 & 0.1713 \\
$\mathbf{7 h}$ & 1.529 & 0.1614 & 2.148 & 0.1649 & 3.367 & 0.1821 \\
$\mathbf{9 h}$ & 3.164 & 0.1754 & 3.746 & 0.1813 & 4.520 & 0.1859 \\
$\mathbf{1 1 h}$ & 5.847 & 0.1874 & 6.218 & 0.1877 & 7.782 & 0.1893 \\
\hline
\end{tabular}

Zeroth order:

\begin{tabular}{|rcccccc|}
\hline time & tCAT^ $^{\wedge}$ & [product] $\mathbf{~ m M}$ & tCAT^0 $^{\wedge}$ & [product] $\mathbf{~ m M}$ & tCAT^0 & [product] $\mathbf{m M}$ \\
$\mathbf{0 m}$ & 0 & 0 & 0 & 0 & 0 & 0 \\
$\mathbf{1 0 m}$ & 0.0675 & 0.01181 & 0.0847 & 0.01378 & 0.14582 & 0.01642 \\
$\mathbf{3 0 m}$ & 0.3129 & 0.06474 & 0.2485 & 0.03794 & 0.203492 & 0.02835 \\
$\mathbf{6 0 m}$ & 0.4380 & 0.08214 & 0.4856 & 0.07840 & 0.6427052 & 0.07093 \\
$\mathbf{9 0 m}$ & 0.5901 & 0.09267 & 0.7479 & 0.1184 & 1.042 & 0.1058 \\
$\mathbf{2 . 5 h}$ & 0.7092 & 0.1256 & 1.140 & 0.1378 & 1.412 & 0.1185 \\
$\mathbf{5 h}$ & 1.002 & 0.1455 & 1.469 & 0.1485 & 2.090 & 0.1435 \\
$\mathbf{7 h}$ & 1.529 & 0.1765 & 2.148 & 0.1589 & 3.367 & 0.1526 \\
$\mathbf{9 h}$ & 3.164 & 0.1918 & 3.746 & 0.1785 & 4.520 & 0.1557 \\
$\mathbf{1 1 h}$ & 5.847 & 0.2050 & 6.218 & 0.1814 & 7.782 & 0.1586 \\
\hline
\end{tabular}

Second order:

\begin{tabular}{|c|c|c|c|c|c|c|}
\hline time & tCAT^2 & [product] mM & tCAT^2 & [product] $\mathrm{mM}$ & tCAT^2 & [product] $\mathrm{mM}$ \\
\hline $0 m$ & 0 & 0 & 0 & 0 & 0 & 0 \\
\hline $10 \mathrm{~m}$ & 0.0675 & 0.01162 & 0.0847 & 0.0141 & 0.14582 & 0.02703 \\
\hline $30 m$ & 0.3129 & 0.02784 & 0.2485 & 0.03394 & 0.203492 & 0.04665 \\
\hline $60 m$ & 0.4380 & 0.049 & 0.4856 & 0.0814 & 0.6427052 & 0.1167 \\
\hline $90 \mathrm{~m}$ & 0.5901 & 0.0746 & 0.7479 & 0.1289 & 1.042 & 0.1742 \\
\hline $2.5 \mathrm{~h}$ & 0.7092 & 0.08974 & 1.140 & 0.1475 & 1.412 & 0.1951 \\
\hline $5 h$ & 1.002 & 0.1238 & 1.469 & 0.1748 & 2.090 & 0.2361 \\
\hline $7 \mathrm{~h}$ & 1.529 & 0.1468 & 2.148 & 0.1938 & 3.367 & 0.2511 \\
\hline $9 h$ & 3.164 & 0.1838 & 3.746 & 0.2090 & 4.520 & 0.2563 \\
\hline $11 \mathrm{~h}$ & 5.847 & 0.1993 & 6.218 & 0.2194 & 7.782 & 0.2610 \\
\hline
\end{tabular}


Determining the order with respect to methyl-2-benzoate

First order:

\begin{tabular}{|c|c|c|c|c|c|c|}
\hline time & tCAT^1 & [product] $\mathrm{mM}$ & tCAT^1 & [product] $\mathrm{mM}$ & tCAT^1 & [product] $\mathrm{mM}$ \\
\hline Om & 0.0000 & 0 & 0 & 0 & 0 & 0 \\
\hline $10 \mathrm{~m}$ & 0.1193 & 0.01989 & 0.2768 & 0.03379 & 0.4195 & 0.0541 \\
\hline $30 m$ & 0.3580 & 0.05967 & 1.121 & 0.1014 & 1.458 & 0.1234 \\
\hline $60 \mathrm{~m}$ & 0.5374 & 0.07966 & 1.612 & 0.1212 & 1.785 & 0.1273 \\
\hline $90 \mathrm{~m}$ & 0.7558 & 0.09818 & 2.220 & 0.1403 & 2.684 & 0.1579 \\
\hline $2.5 \mathrm{~h}$ & 0.9694 & 0.1040 & 3.226 & 0.1549 & 3.749 & 0.17 \\
\hline $5 \mathrm{~h}$ & 1.508 & 0.1243 & 4.523 & 0.1809 & 4.920 & 0.1846 \\
\hline $7 \mathrm{~h}$ & 1.874 & 0.1415 & 6.445 & 0.1982 & 7.195 & 0.2018 \\
\hline $9 \mathrm{~h}$ & 4.163 & 0.1757 & 8.795 & 0.1984 & 9.479 & 0.2084 \\
\hline $11 \mathrm{~h}$ & 8.218 & 0.2046 & 12.49 & 0.2037 & 14.68 & 0.2140 \\
\hline
\end{tabular}

Zeroth order:

\begin{tabular}{|rcccccc|}
\hline time & tCAT^ $^{\mathbf{0}}$ & [product] $\mathbf{~ m M}$ & tCAT $^{\wedge} \mathbf{0}$ & [product] $\mathbf{~ m M}$ & tCAT^0 $^{\wedge}$ & [product] $\mathbf{~ m M ~}$ \\
$\mathbf{0 m}$ & 0.0000 & 0 & 0 & 0 & 0 & 0 \\
$\mathbf{1 0 m}$ & 0.1193 & 0.01899 & 0.2768 & 0.08264 & 0.4195 & 0.034 \\
$\mathbf{3 0 m}$ & 0.3580 & 0.05696 & 1.121 & 0.2479 & 1.458 & 0.0547 \\
$\mathbf{6 0 m}$ & 0.5374 & 0.07604 & 1.612 & 0.3722 & 1.785 & 0.06998 \\
$\mathbf{9 0 m}$ & 0.7558 & 0.09372 & 2.220 & 0.5235 & 2.684 & 0.08164 \\
$\mathbf{2 . 5 h}$ & 0.9694 & 0.0993 & 3.226 & 0.6715 & 3.749 & 0.091 \\
$\mathbf{5 h}$ & 1.508 & 0.1187 & 4.523 & 1.0444 & 4.920 & 0.1047 \\
$\mathbf{7 h}$ & 1.874 & 0.1350 & 6.445 & 1.2977 & 7.195 & 0.1198 \\
$\mathbf{9 h}$ & 4.163 & 0.1677 & 8.795 & 3.3001 & 9.479 & 0.1294 \\
$\mathbf{1 1 h}$ & 8.218 & 0.1953 & 12.49 & 6.5138 & 14.68 & 0.1479 \\
\hline
\end{tabular}

Second order:

\begin{tabular}{|c|c|c|c|c|c|c|}
\hline time & tCAT^2 & [product] $\mathrm{mM}$ & tCAT^2 & [product] $\mathrm{mM}$ & tCAT^2 & [product] $\mathrm{mM}$ \\
\hline Om & 0.0000 & 0 & 0 & 0 & 0 & 0 \\
\hline $10 \mathrm{~m}$ & 0.1193 & 0.01591 & 0.2768 & 0.04003 & 0.4195 & 0.034 \\
\hline $30 \mathrm{~m}$ & 0.3580 & 0.04772 & 1.121 & 0.1201 & 1.458 & 0.09367 \\
\hline $60 \mathrm{~m}$ & 0.5374 & 0.06370 & 1.612 & 0.1436 & 1.785 & 0.1175 \\
\hline $90 \mathrm{~m}$ & 0.7558 & 0.06957 & 2.220 & 0.1662 & 2.684 & 0.1374 \\
\hline $2.5 \mathrm{~h}$ & 0.9694 & 0.0831 & 3.226 & 0.1835 & 3.749 & 0.18 \\
\hline $5 \mathrm{~h}$ & 1.508 & 0.0994 & 4.523 & 0.1990 & 4.920 & 0.21847 \\
\hline $7 \mathrm{~h}$ & 1.874 & 0.1131 & 6.445 & 0.2181 & 7.195 & 0.2477 \\
\hline $9 h$ & 4.163 & 0.1573 & 8.795 & 0.2282 & 9.479 & 0.2765 \\
\hline $11 \mathrm{~h}$ & 8.218 & 0.1724 & 12.49 & 0.2404 & 14.68 & 0.2947 \\
\hline
\end{tabular}




\section{NMR Spectra}

1-Methyl-4-phenylbenzene $3\left(\mathrm{CDCl}_{3}, 400 \mathrm{MHz}\right.$ for ${ }^{1} \mathrm{H} \mathrm{NMR}, 100 \mathrm{MHz}$ for $\left.{ }^{13} \mathrm{C} \mathrm{NMR}\right)$

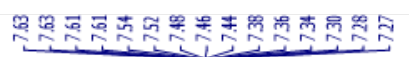<smiles>Cc1ccc(-c2ccccc2)cc1</smiles>

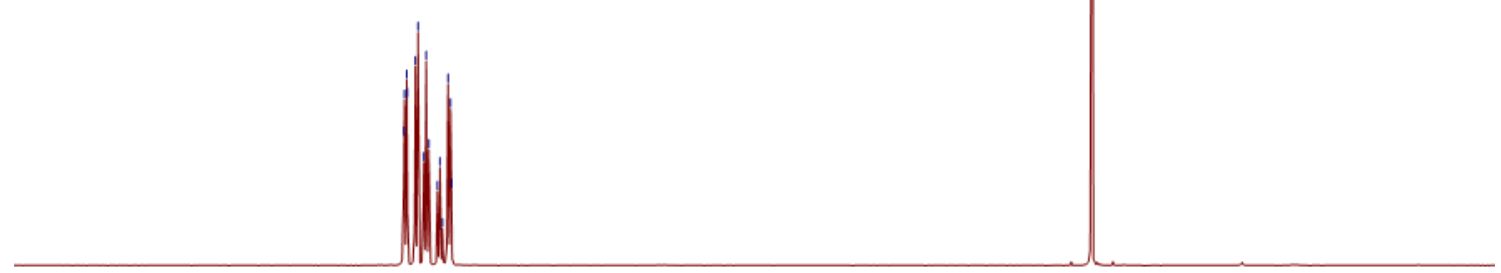

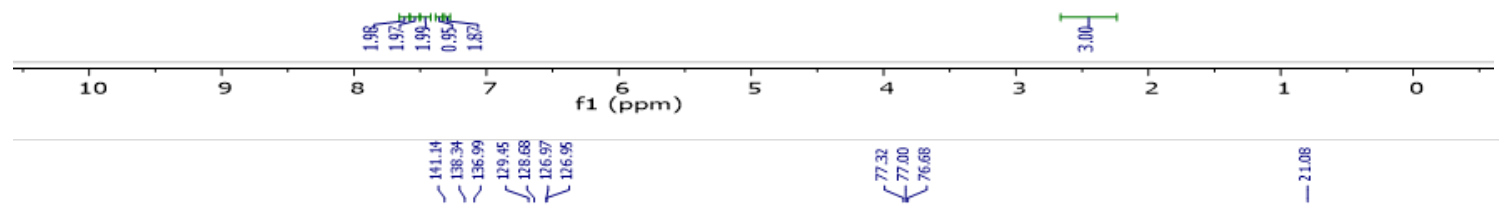
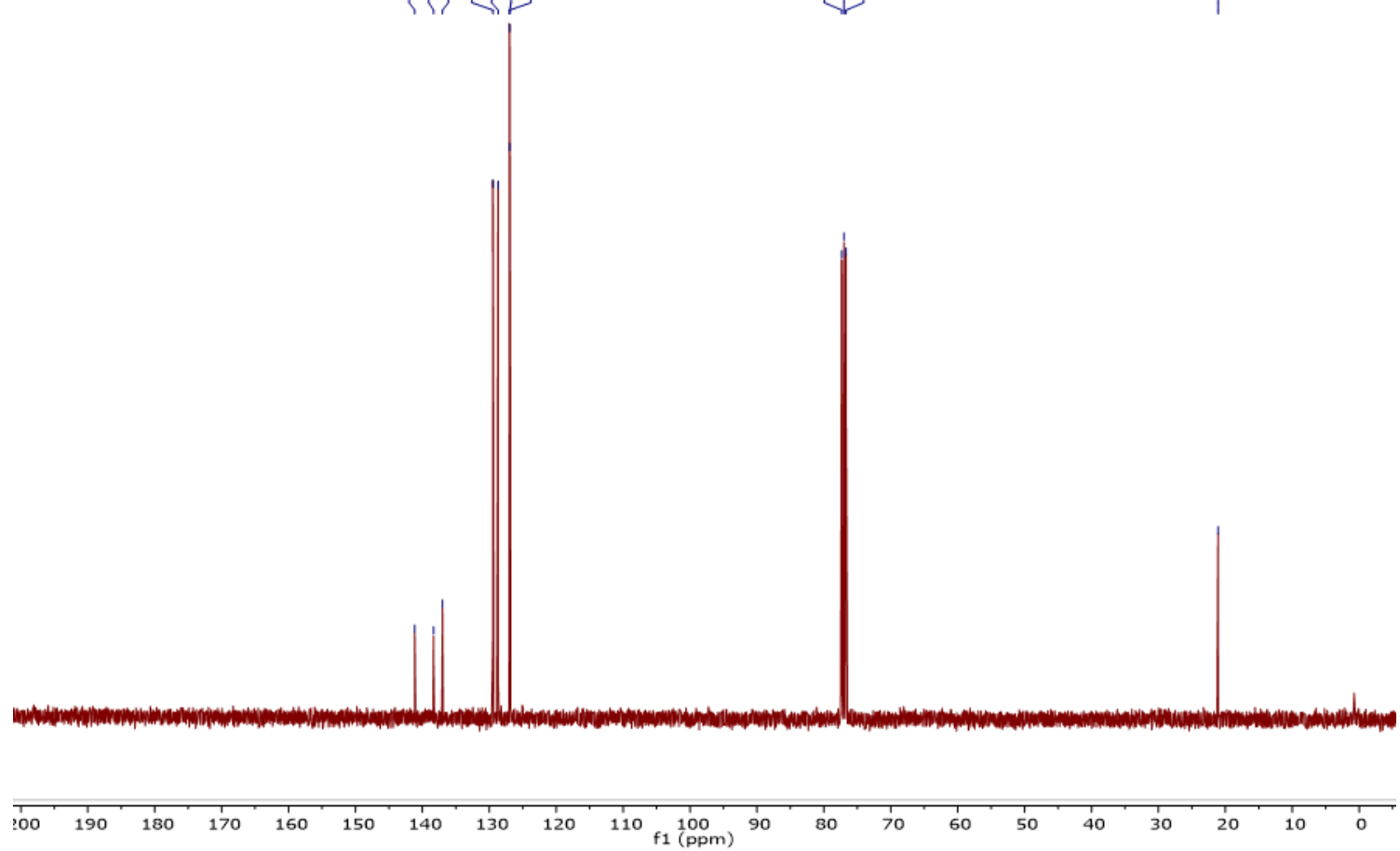
2-Methylnaphthalene $4\left(\mathrm{CDCl}_{3}, 400 \mathrm{MHz}\right.$ for ${ }^{1} \mathrm{H} \mathrm{NMR}, 100 \mathrm{MHz}$ for ${ }^{13} \mathrm{C} \mathrm{NMR}$ )

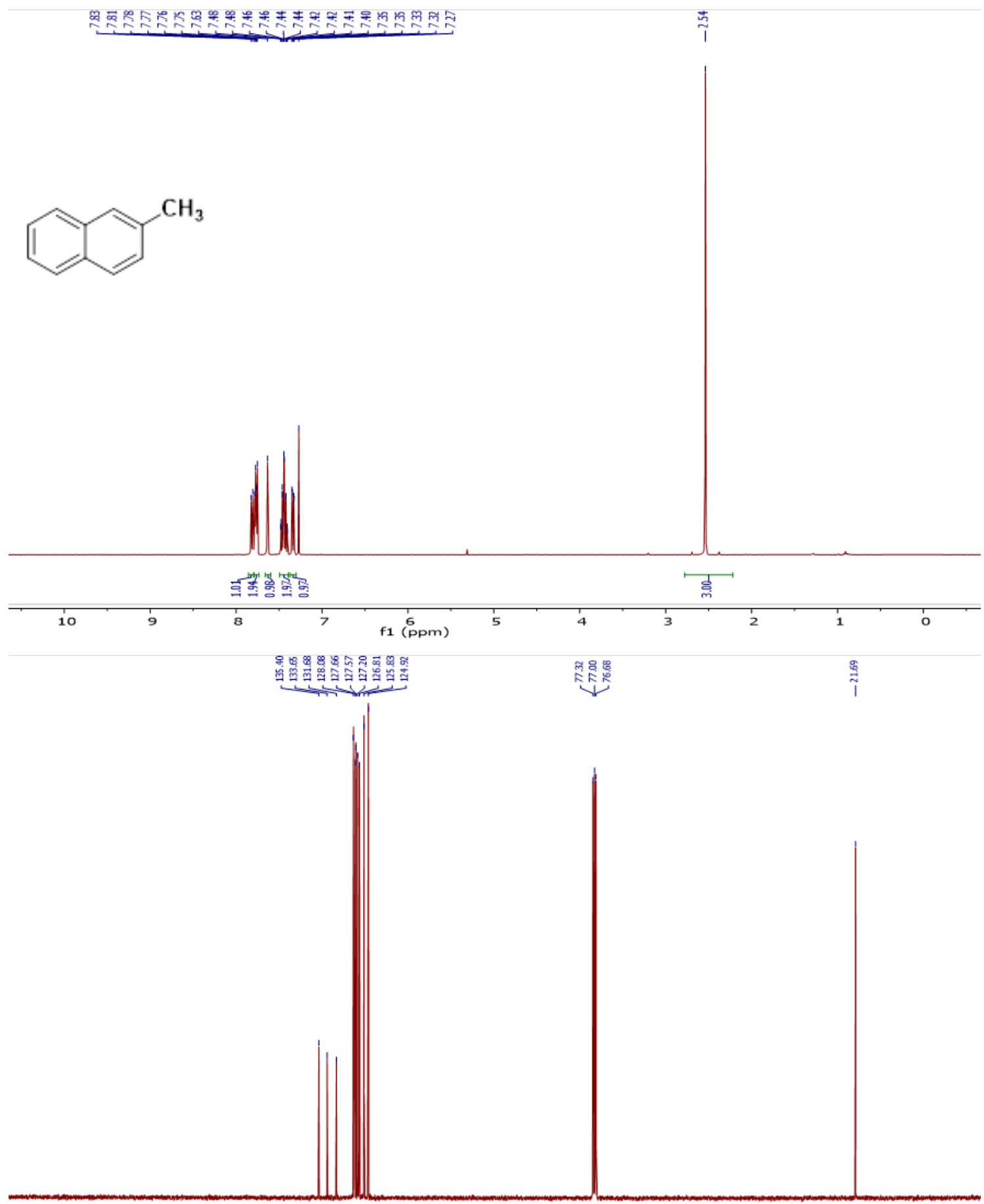

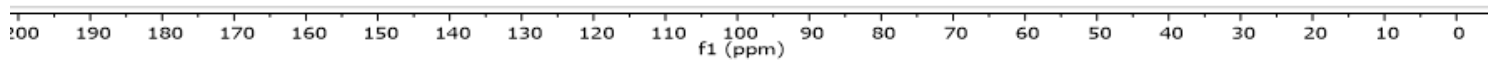


9-Methylphenanthrene $5\left(\mathrm{CDCl}_{3}, 400 \mathrm{MHz}\right.$ for ${ }^{1} \mathrm{H} \mathrm{NMR}, 100 \mathrm{MHz}$ for $\left.{ }^{13} \mathrm{C} \mathrm{NMR}\right)$<smiles>Cc1cc2ccccc2c2ccccc12</smiles>
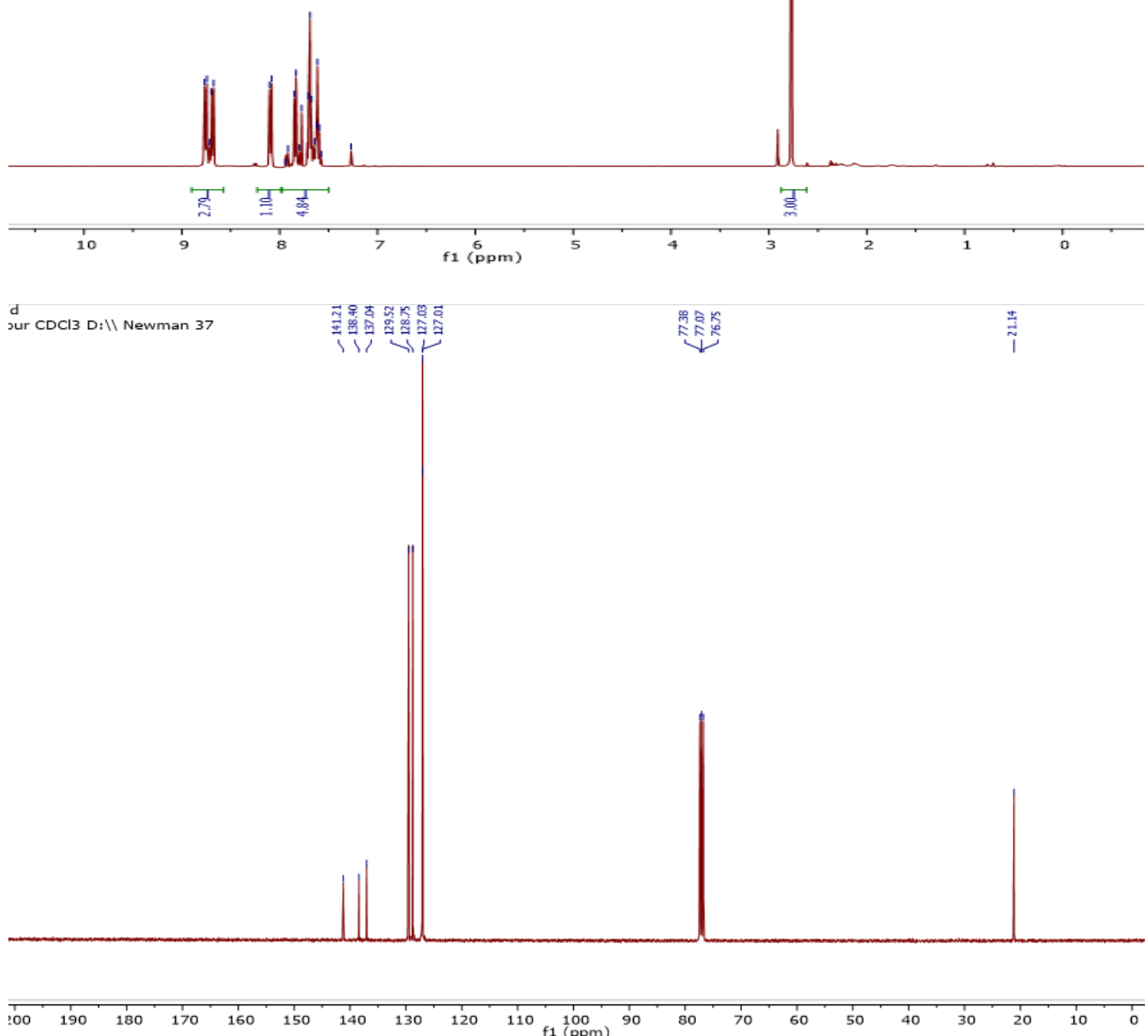
1-(trans-4-butylcyclohexyl)-4-methylbenzene $6\left(\mathrm{CDCl}_{3}, 400 \mathrm{MHz}\right.$ for ${ }^{1} \mathrm{H} \mathrm{NMR}, 100 \mathrm{MHz}$ for $\left.{ }^{13} \mathrm{C} \mathrm{NMR}\right)$

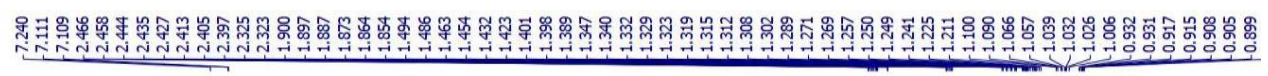
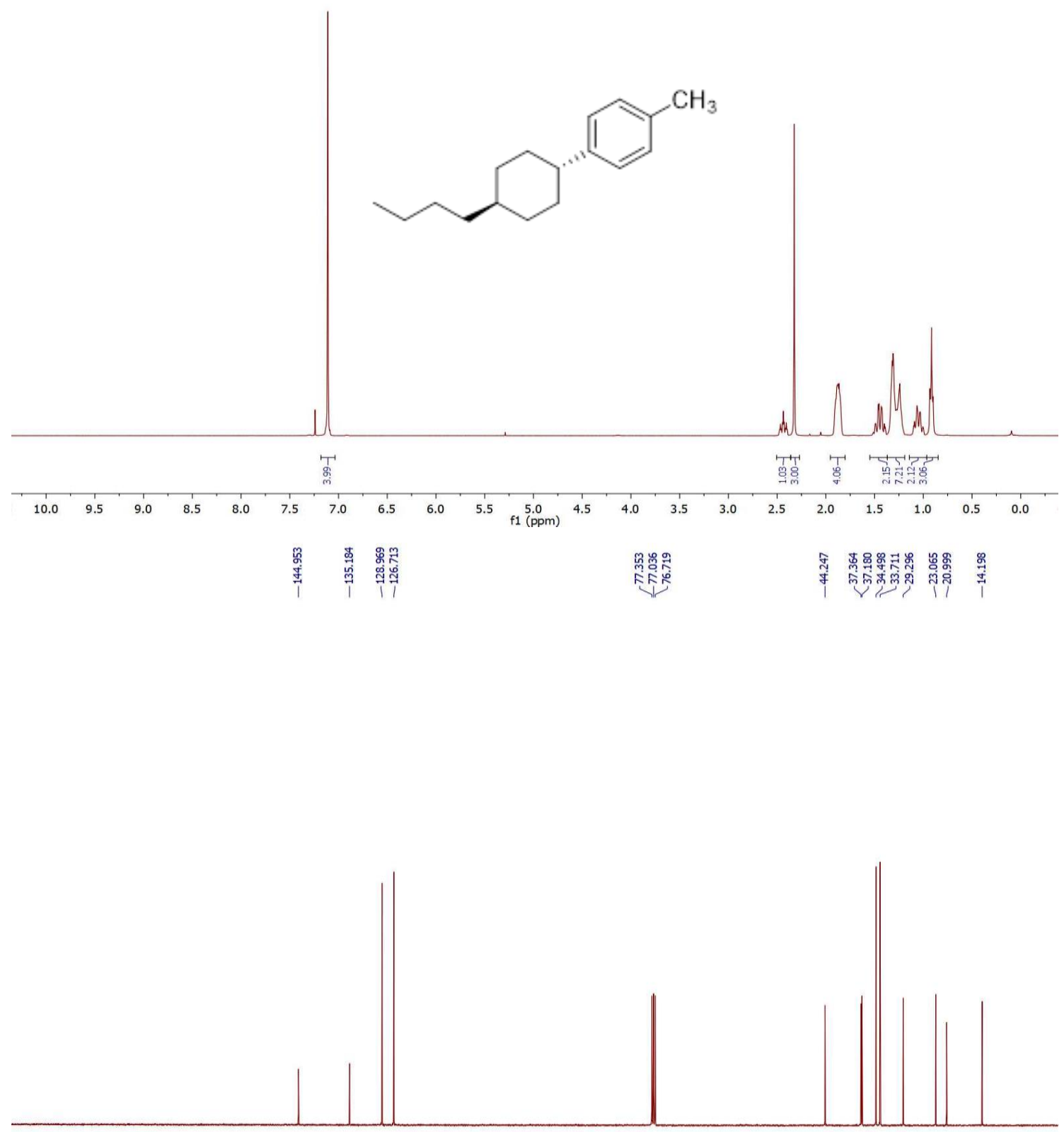
1-Methyl 4-dimethylamino benzene $7\left(\mathrm{CDCl}_{3}, 400 \mathrm{MHz}\right.$ for ${ }^{1} \mathrm{H} \mathrm{NMR}, 100 \mathrm{MHz}$ for $\left.{ }^{13} \mathrm{C} \mathrm{NMR}\right)$
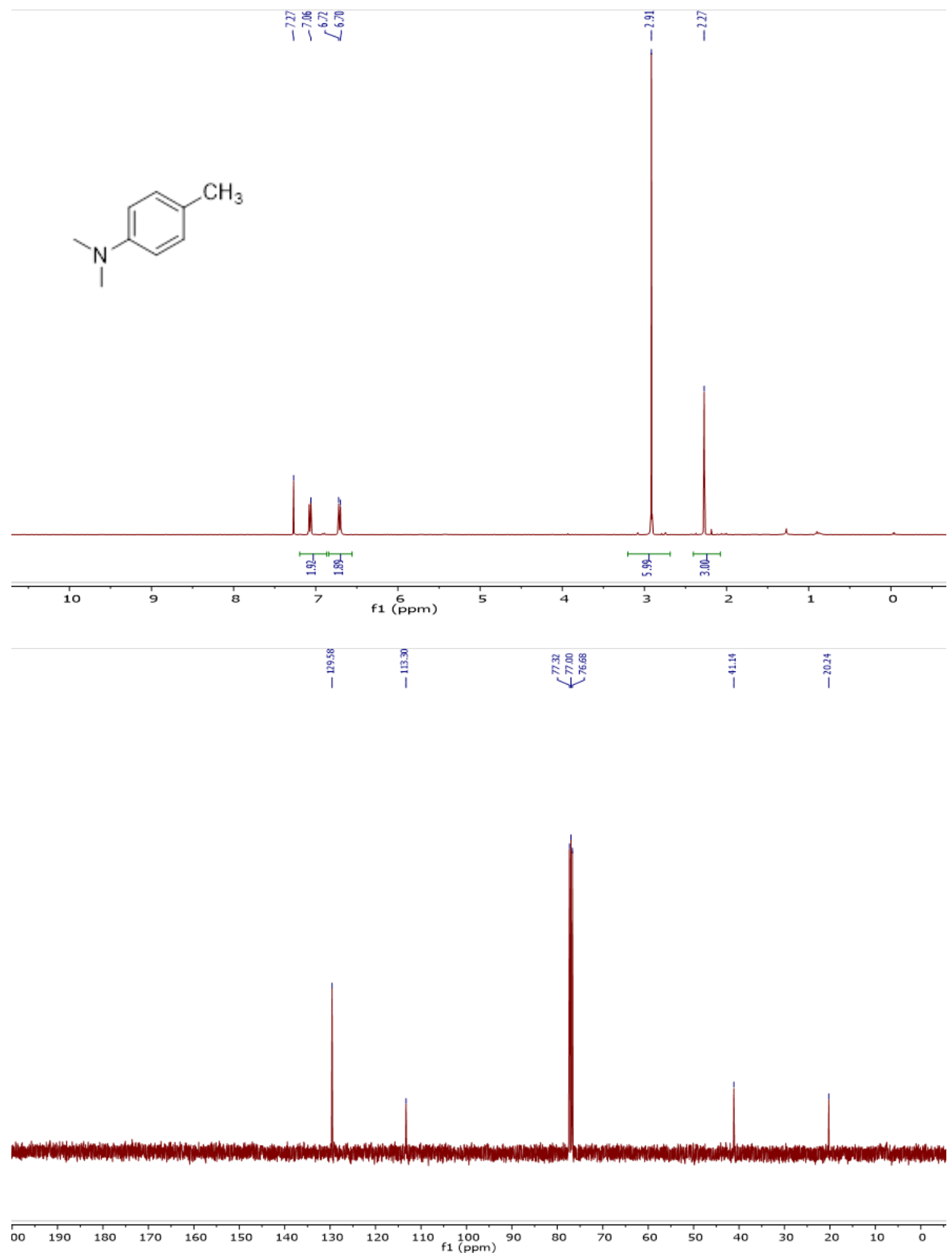
1-Methyl-4-vinylbenzene $8\left(\mathrm{CDCl}_{3}, 400 \mathrm{MHz}\right.$ for ${ }^{1} \mathrm{H} \mathrm{NMR}, 100 \mathrm{MHz}$ for $\left.{ }^{13} \mathrm{C} \mathrm{NMR}\right)$
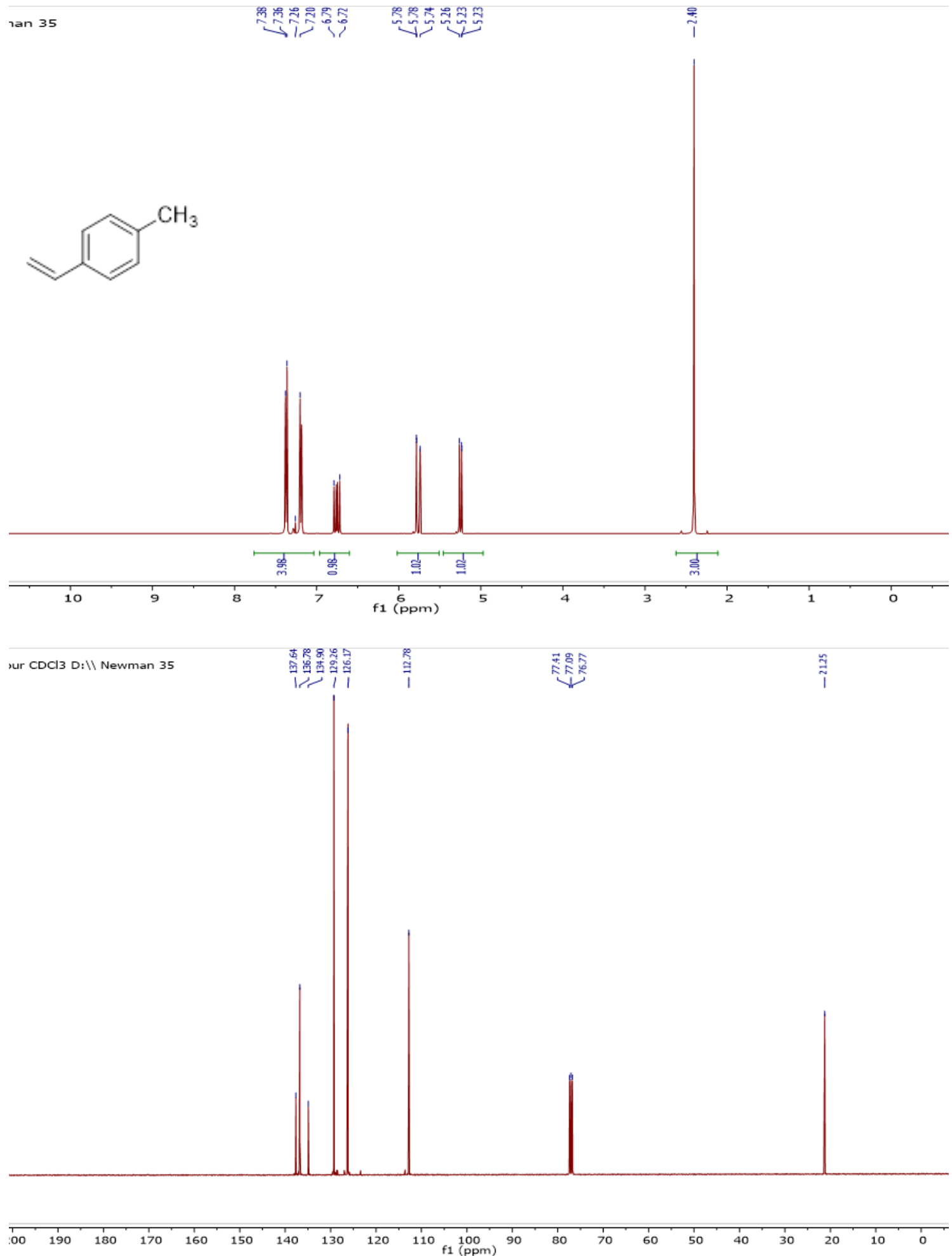
1-Methyl-4-(2-phenylethenyl)benzene 9 ( $\mathrm{CDCl}_{3}, 400 \mathrm{MHz}$ for ${ }^{1} \mathrm{H}$ NMR, $100 \mathrm{MHz}$ for ${ }^{13} \mathrm{C} \mathrm{NMR}$ )<smiles>Cc1ccc(/C=C/c2ccccc2)cc1</smiles>
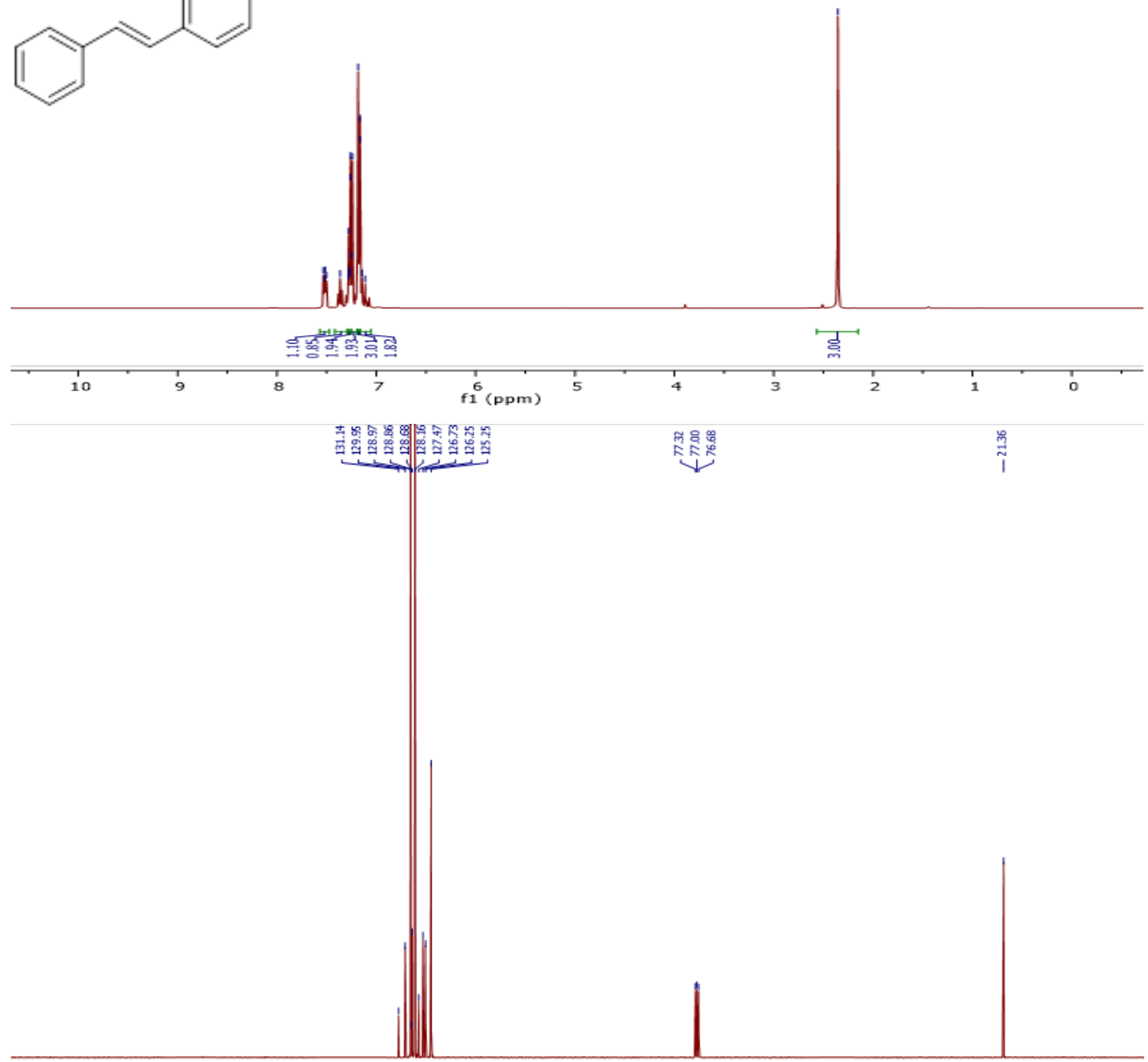

200

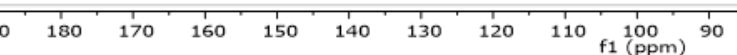

807060 
1,2,3-Trimethoxy-5-methylbenzene $10\left(\mathrm{CDCl}_{3}, 400 \mathrm{MHz}\right.$ for ${ }^{1} \mathrm{H} \mathrm{NMR}, 100 \mathrm{MHz}$ for $\left.{ }^{13} \mathrm{C} \mathrm{NMR}\right)$
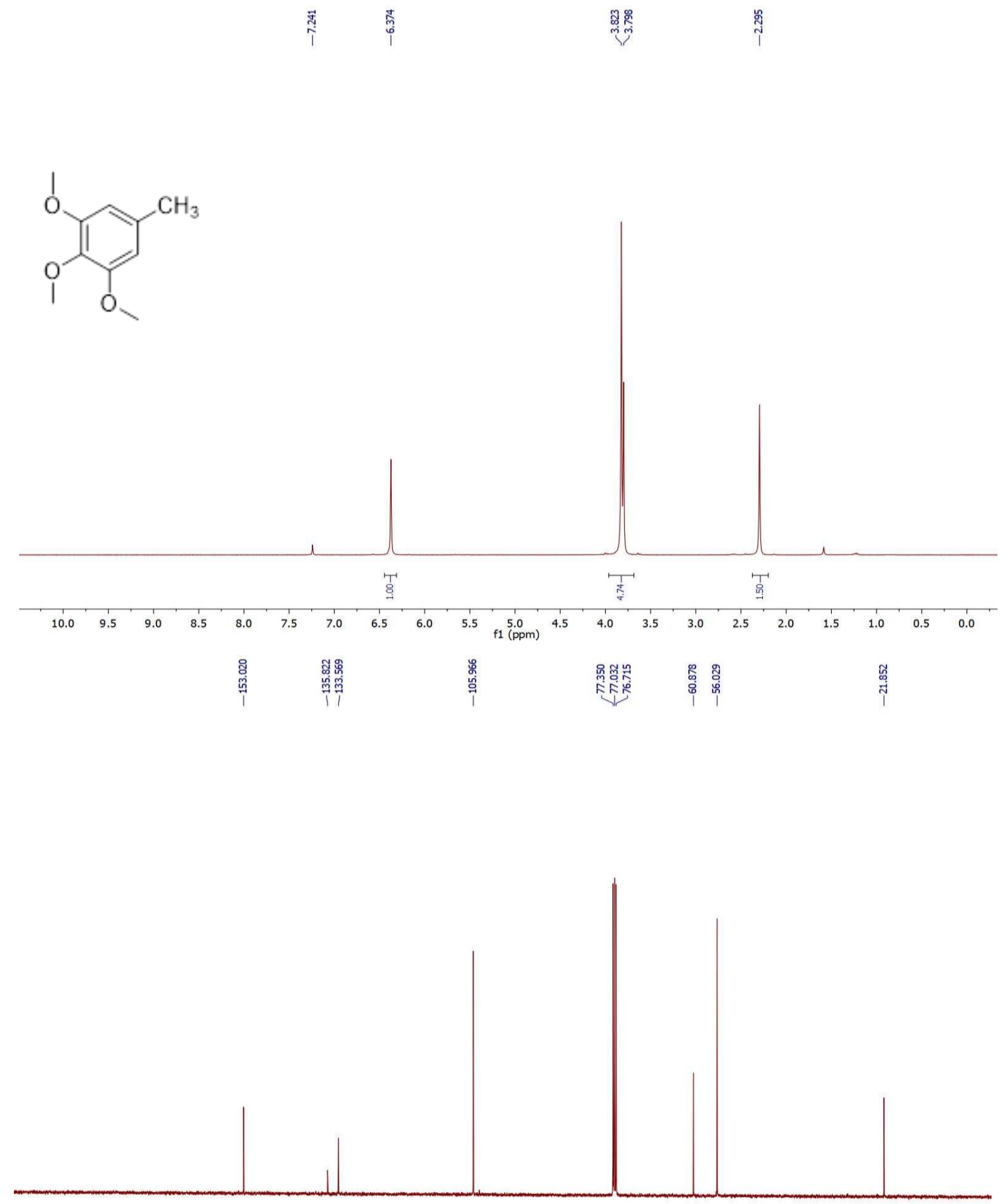
1-Methyl-4-phenoxybenzene 11 ( $\mathrm{CDCl}_{3}, 400 \mathrm{MHz}$ for ${ }^{1} \mathrm{H} \mathrm{NMR}, 100 \mathrm{MHz}$ for ${ }^{13} \mathrm{C} \mathrm{NMR}$ )

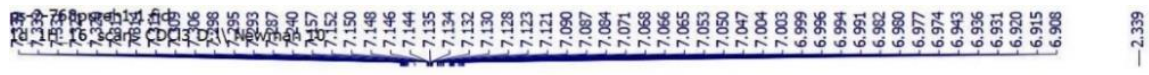
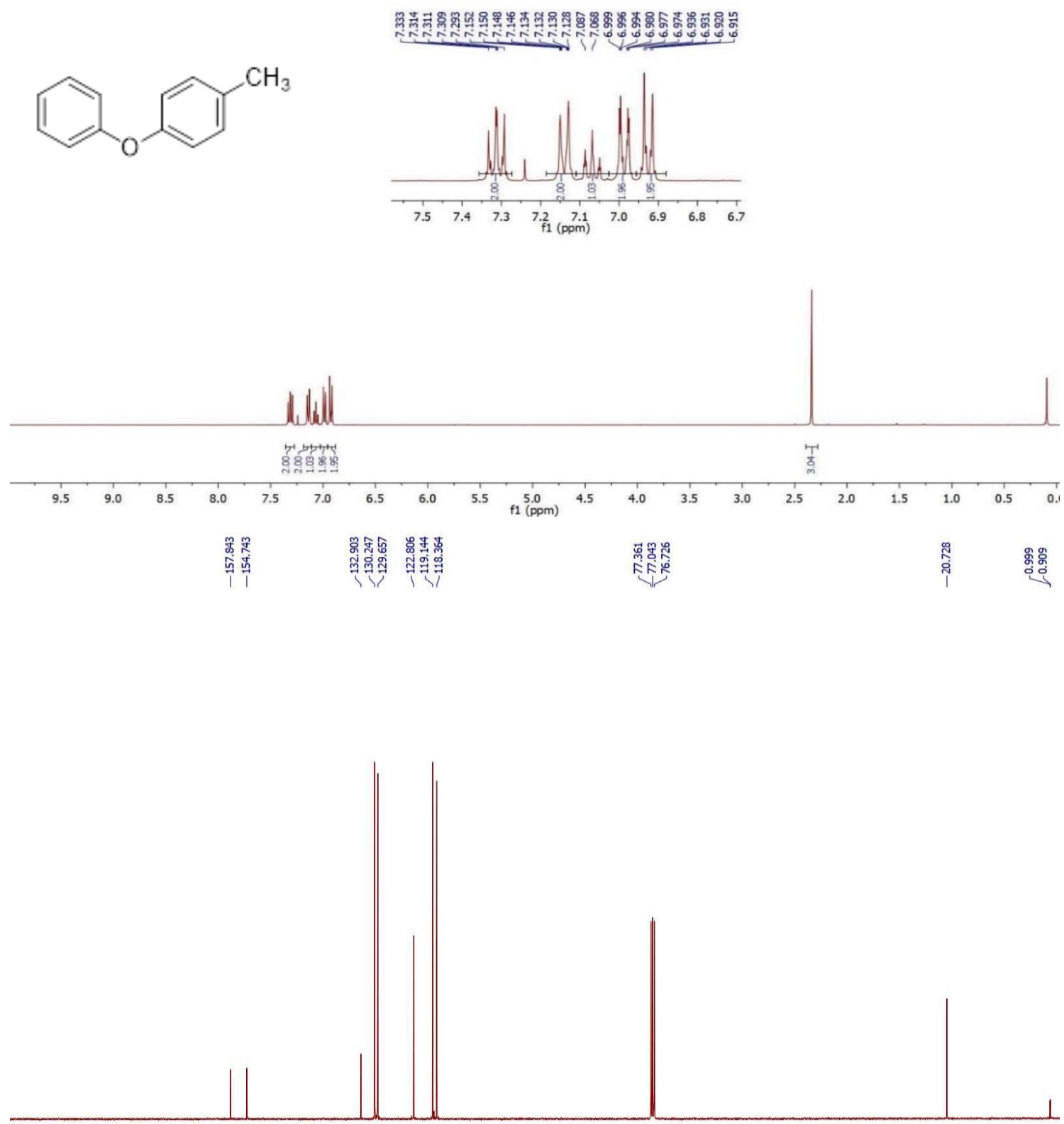
1-Methoxy-4-(4-methylphenoxy)benzene $12\left(\mathrm{CDCl}_{3}, 400 \mathrm{MHz}\right.$ for ${ }^{1} \mathrm{H} \mathrm{NMR}, 100 \mathrm{MHz}$ for $\left.{ }^{13} \mathrm{C} \mathrm{NMR}\right)$

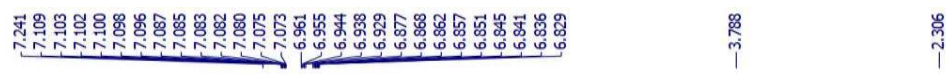<smiles>COc1ccc(Oc2ccc(C)cc2)cc1</smiles>

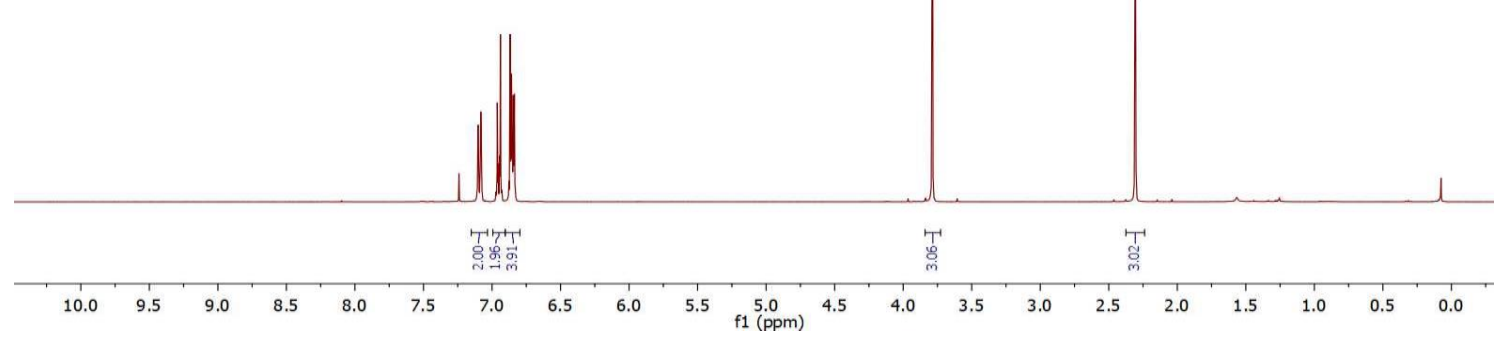

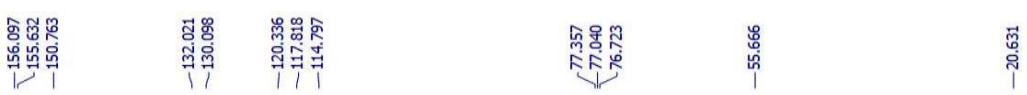

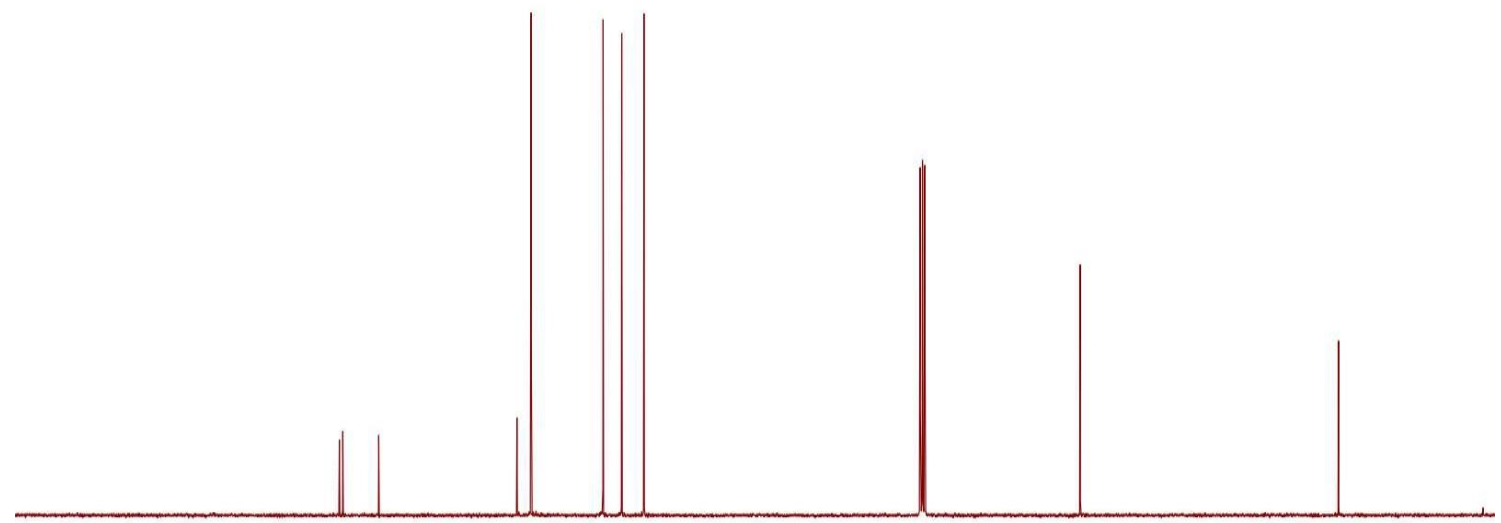


1-(4-Methylphenoxy)-4-phenoxybenzene $13\left(\mathrm{CDCl}_{3}, 400 \mathrm{MHz}\right.$ for ${ }^{1} \mathrm{H} \mathrm{NMR}, 100 \mathrm{MHz}$ for $\left.{ }^{13} \mathrm{C} \mathrm{NMR}\right)$

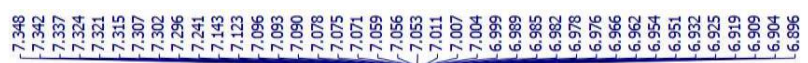<smiles>Cc1ccc(Oc2ccc(Oc3ccccc3)cc2)cc1</smiles>
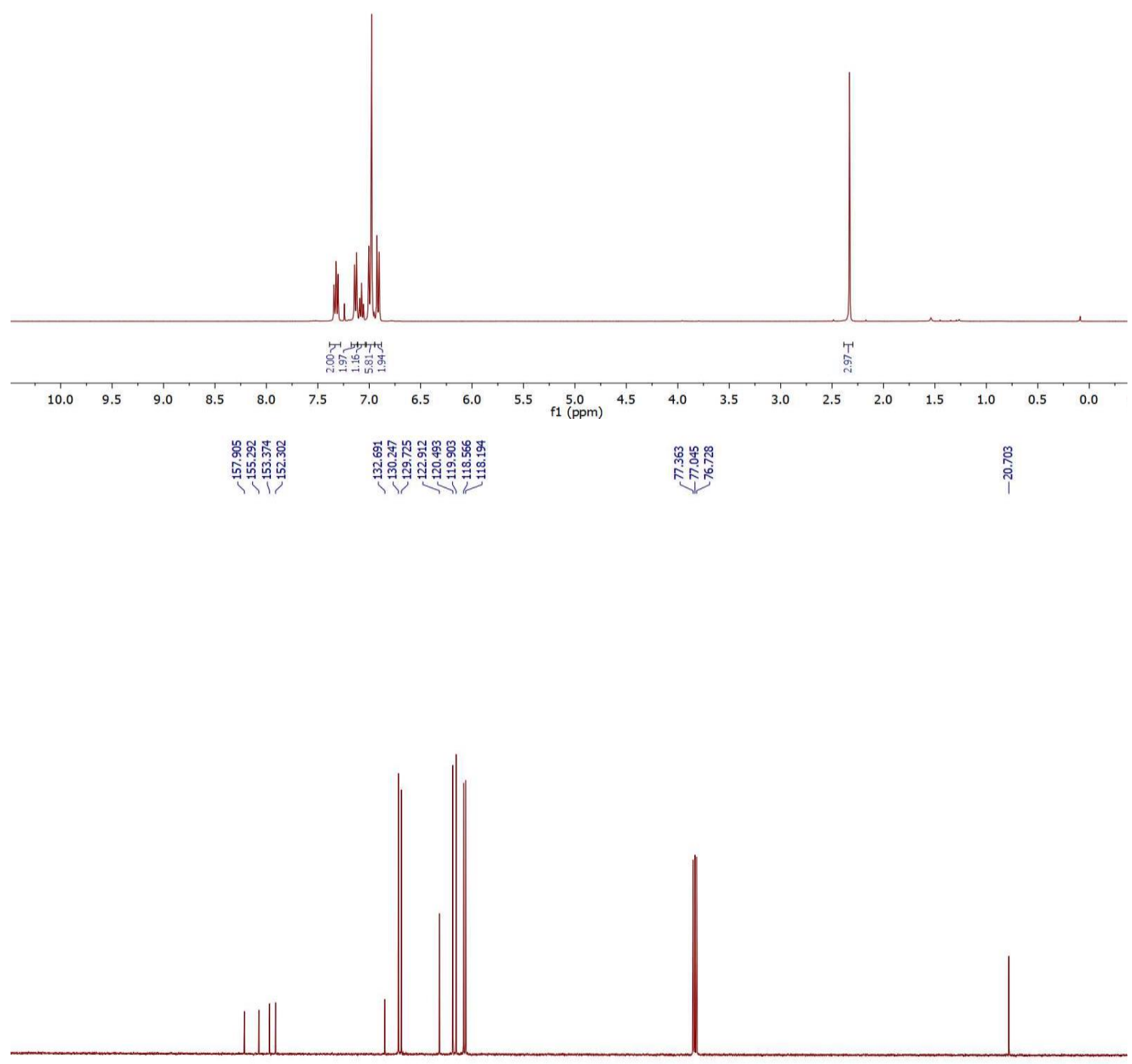
4'-Methyl-[1,1'-biphenyl]-2-ol 14 ( $\mathrm{CDCl}_{3}, 400 \mathrm{MHz}$ for ${ }^{1} \mathrm{H}$ NMR, $100 \mathrm{MHz}$ for $\left.{ }^{13} \mathrm{C} \mathrm{NMR}\right)$<smiles>Cc1ccc(-c2ccccc2O)cc1</smiles>

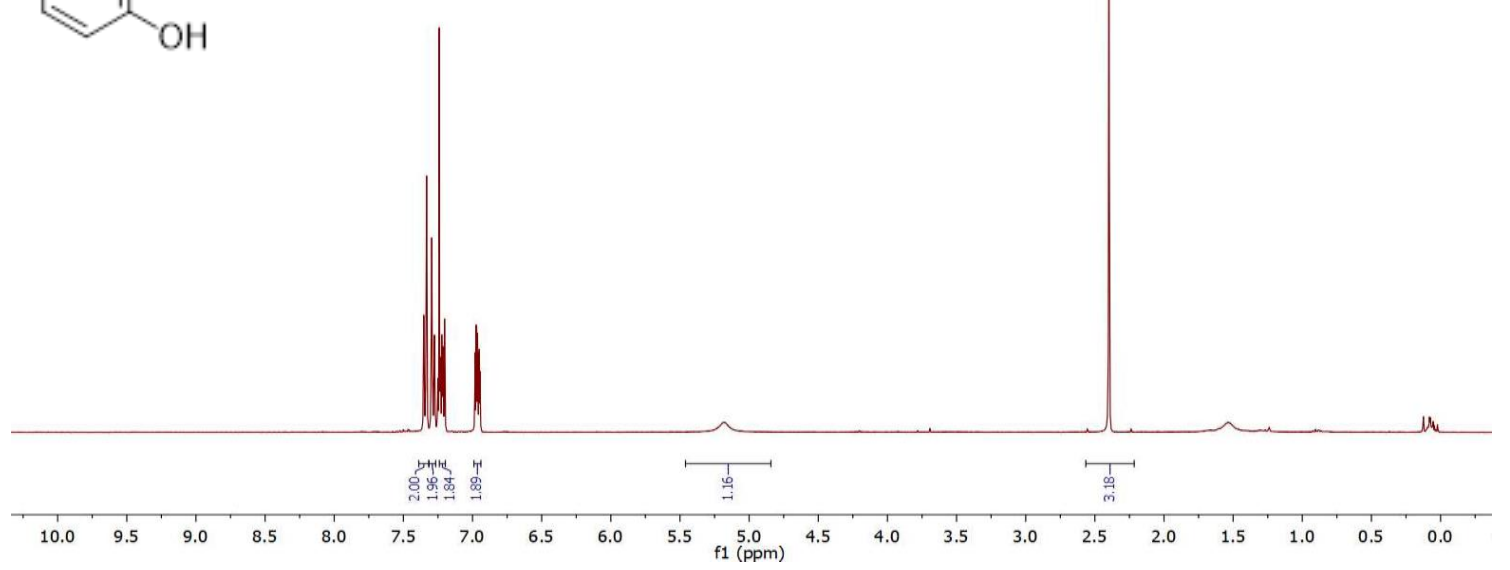

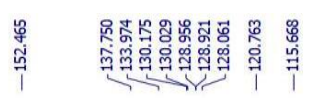
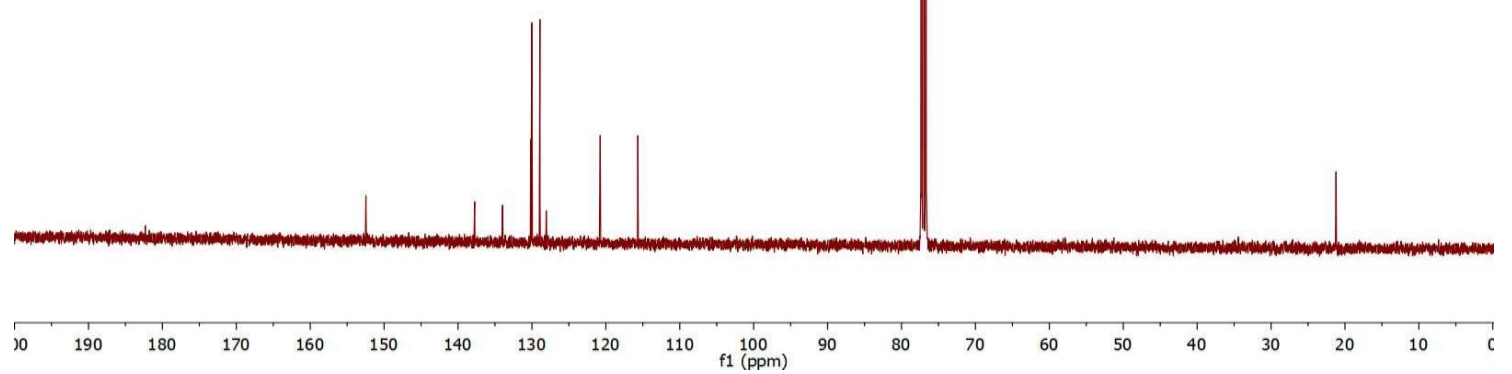
2,6-Bis(1,1-dimethylethyl)-4-methylphenol 15 ( $\mathrm{CDCl}_{3}, 400 \mathrm{MHz}$ for ${ }^{1} \mathrm{H} \mathrm{NMR}, 100 \mathrm{MHz}$ for $\left.{ }^{13} \mathrm{C} \mathrm{NMR}\right)$
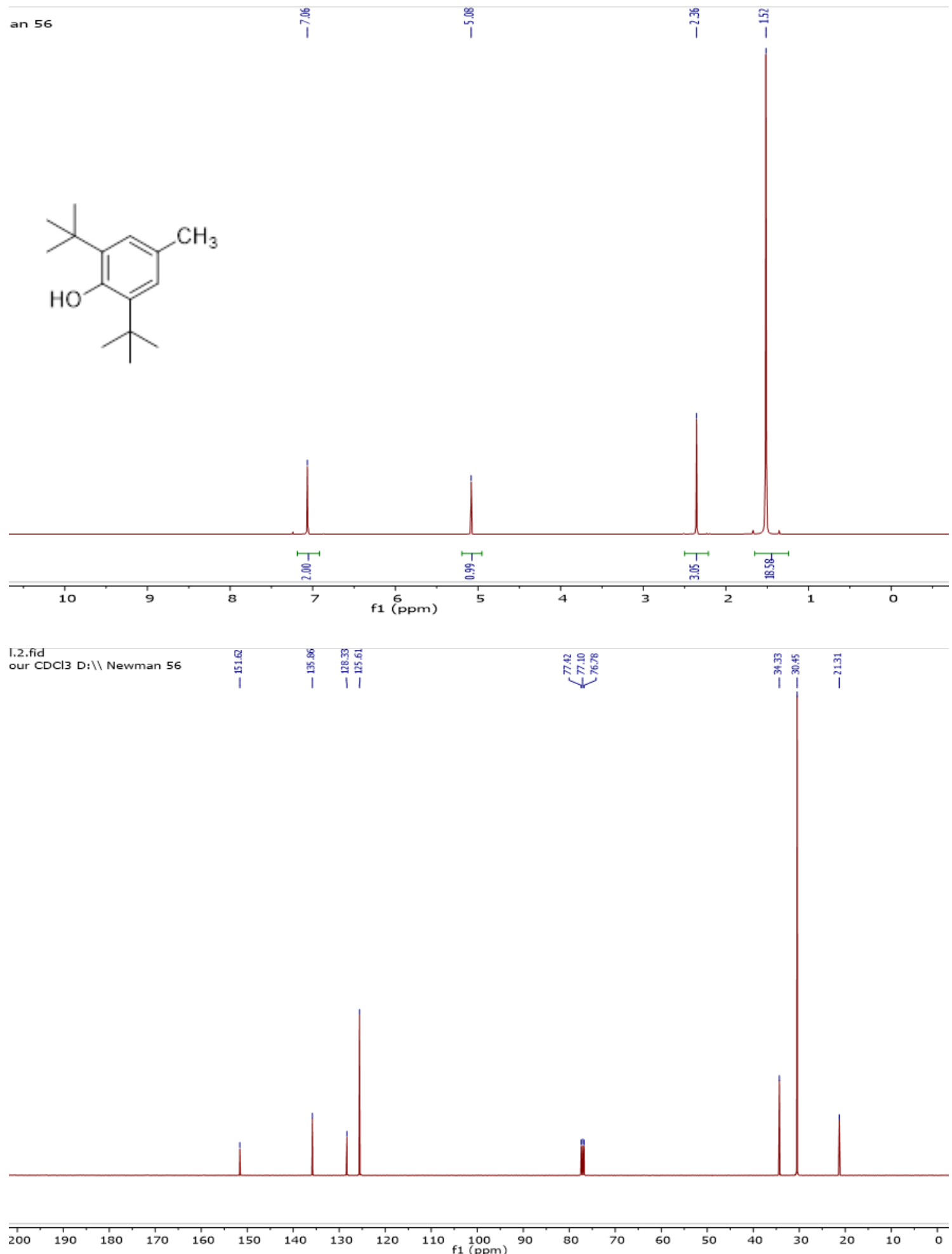
4'-Methyl-4-biphenylcarboxylic acid 16 (CDCl, $400 \mathrm{MHz}$ for ${ }^{1} \mathrm{H}$ NMR, $100 \mathrm{MHz}$ for $\left.{ }^{13} \mathrm{C} \mathrm{NMR}\right)$

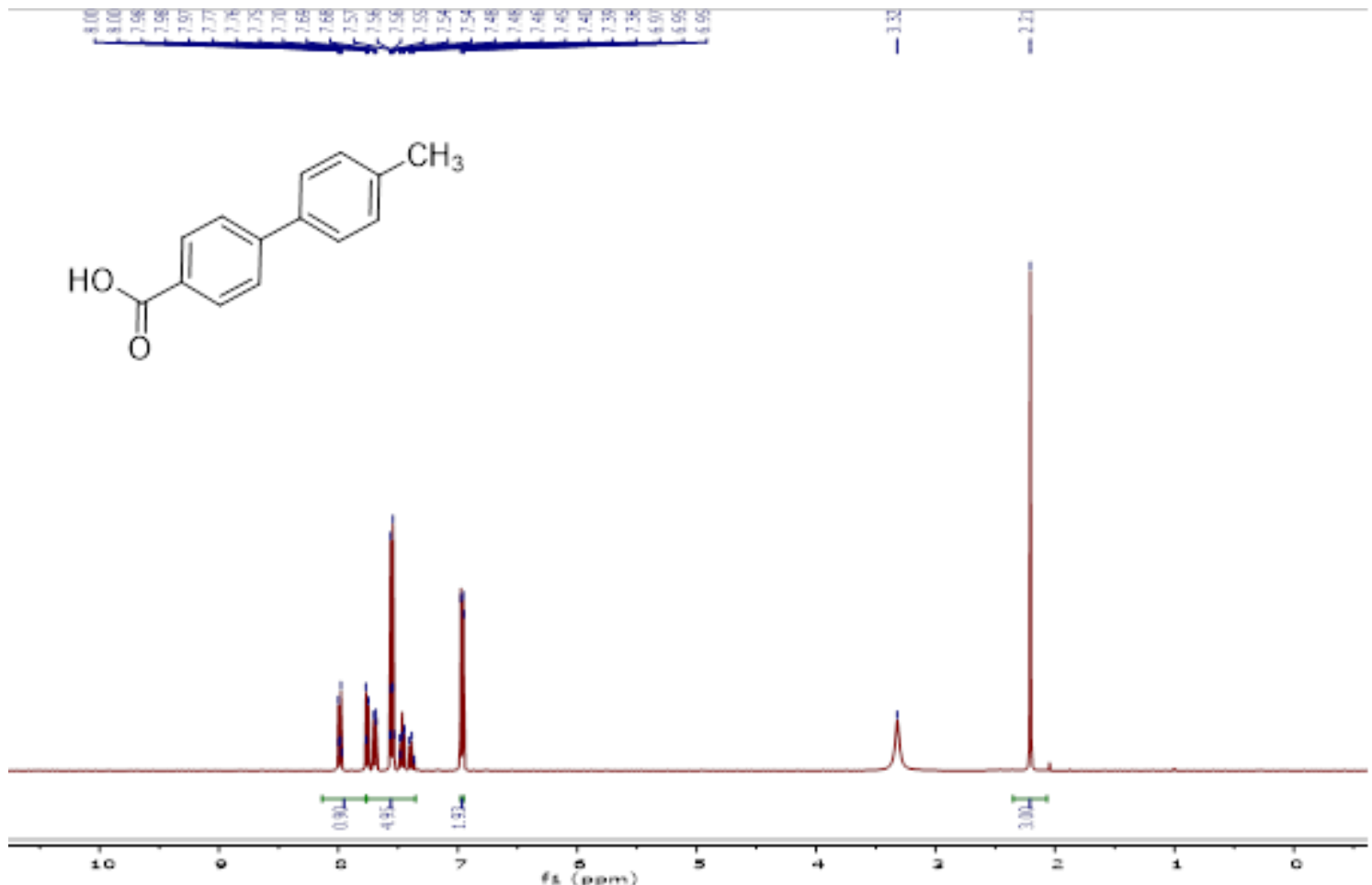

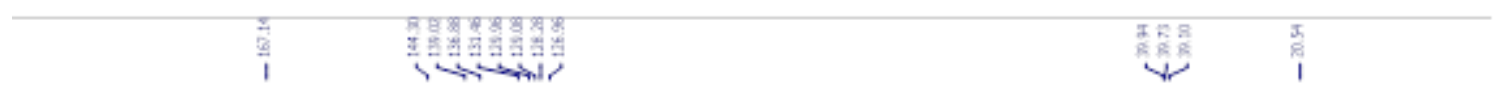
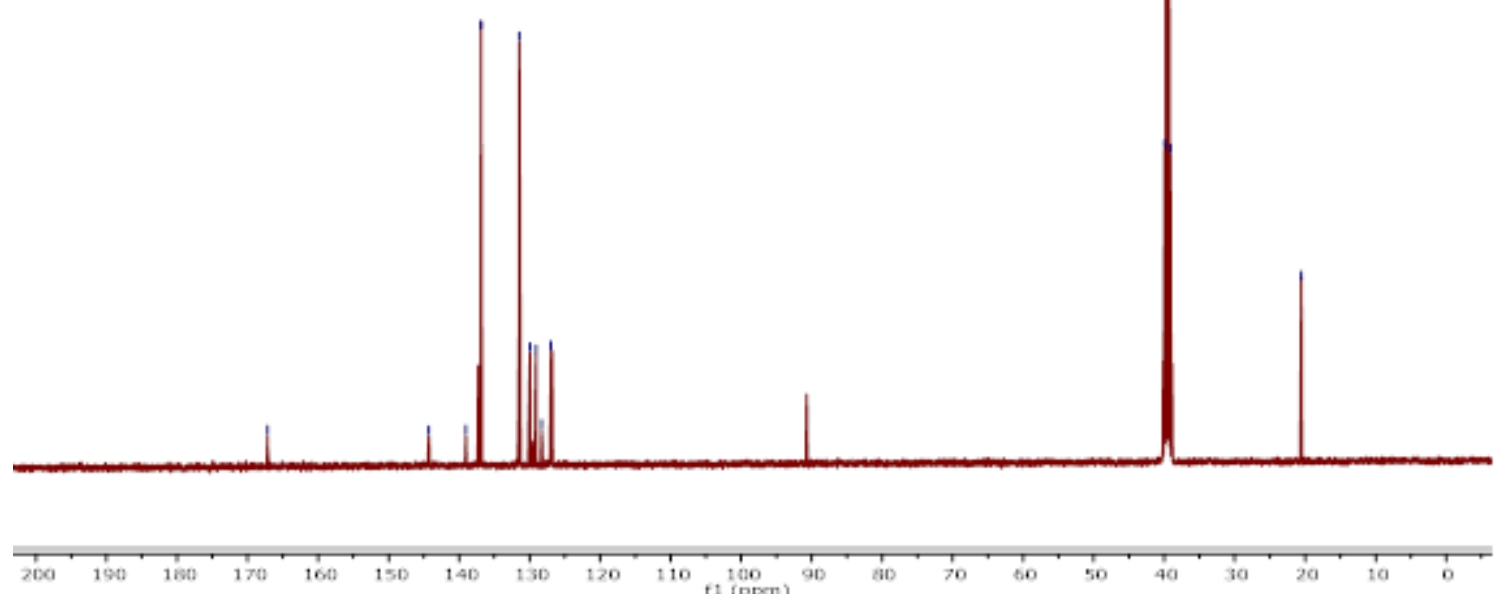
5-Methyl-2-phenylpyridine 17 ( $\mathrm{CDCl}_{3}, 400 \mathrm{MHz}$ for ${ }^{1} \mathrm{H} \mathrm{NMR}, 100 \mathrm{MHz}$ for $\left.{ }^{13} \mathrm{C} \mathrm{NMR}\right)$

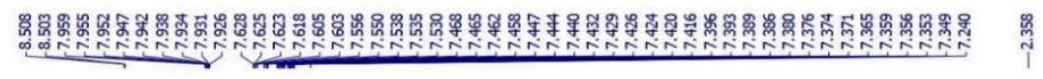<smiles>Cc1ccc(-c2ccccc2)nc1</smiles>

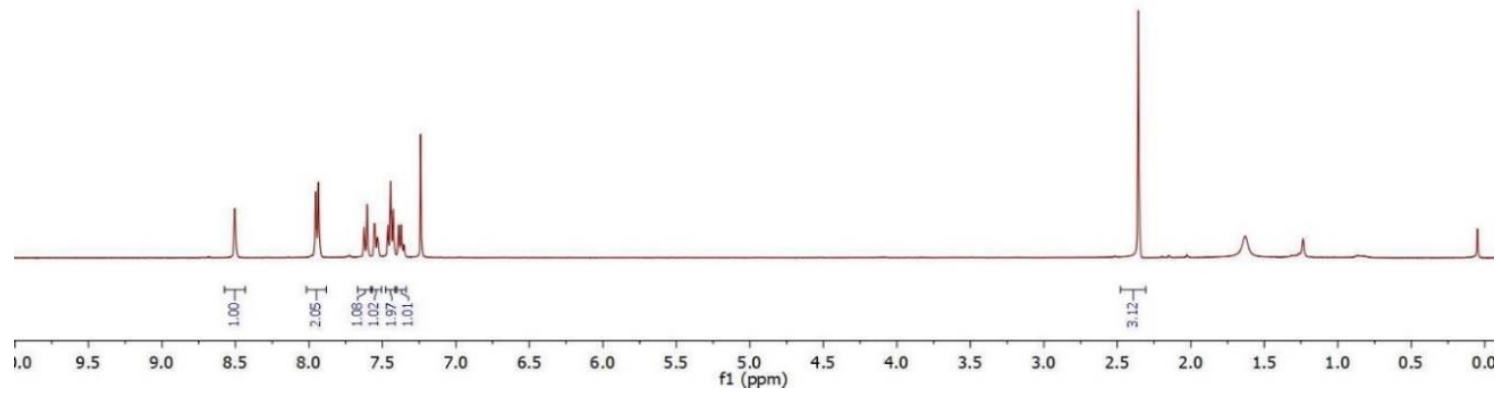

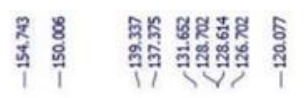

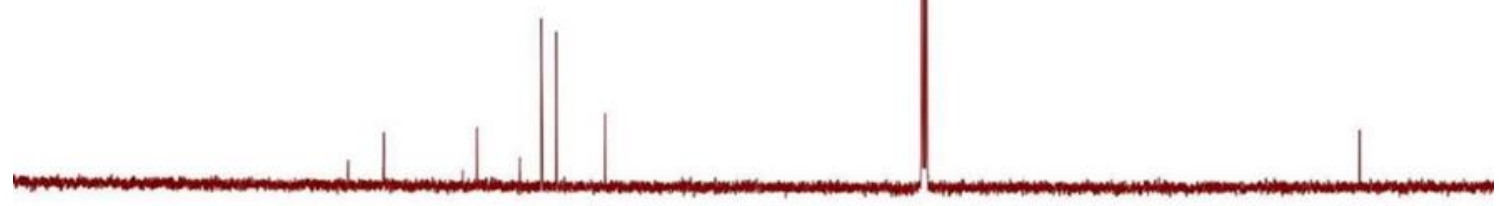

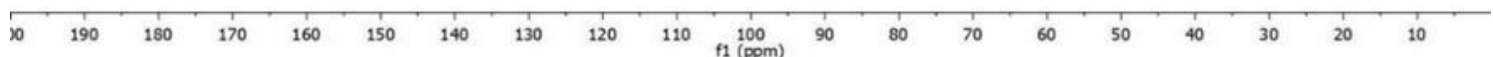


2-(p-Tolyl)pyridine $18\left(\mathrm{CDCl}_{3}, 400 \mathrm{MHz}\right.$ for ${ }^{1} \mathrm{H} \mathrm{NMR}, 100 \mathrm{MHz}$ for $\left.{ }^{13} \mathrm{C} \mathrm{NMR}\right)$
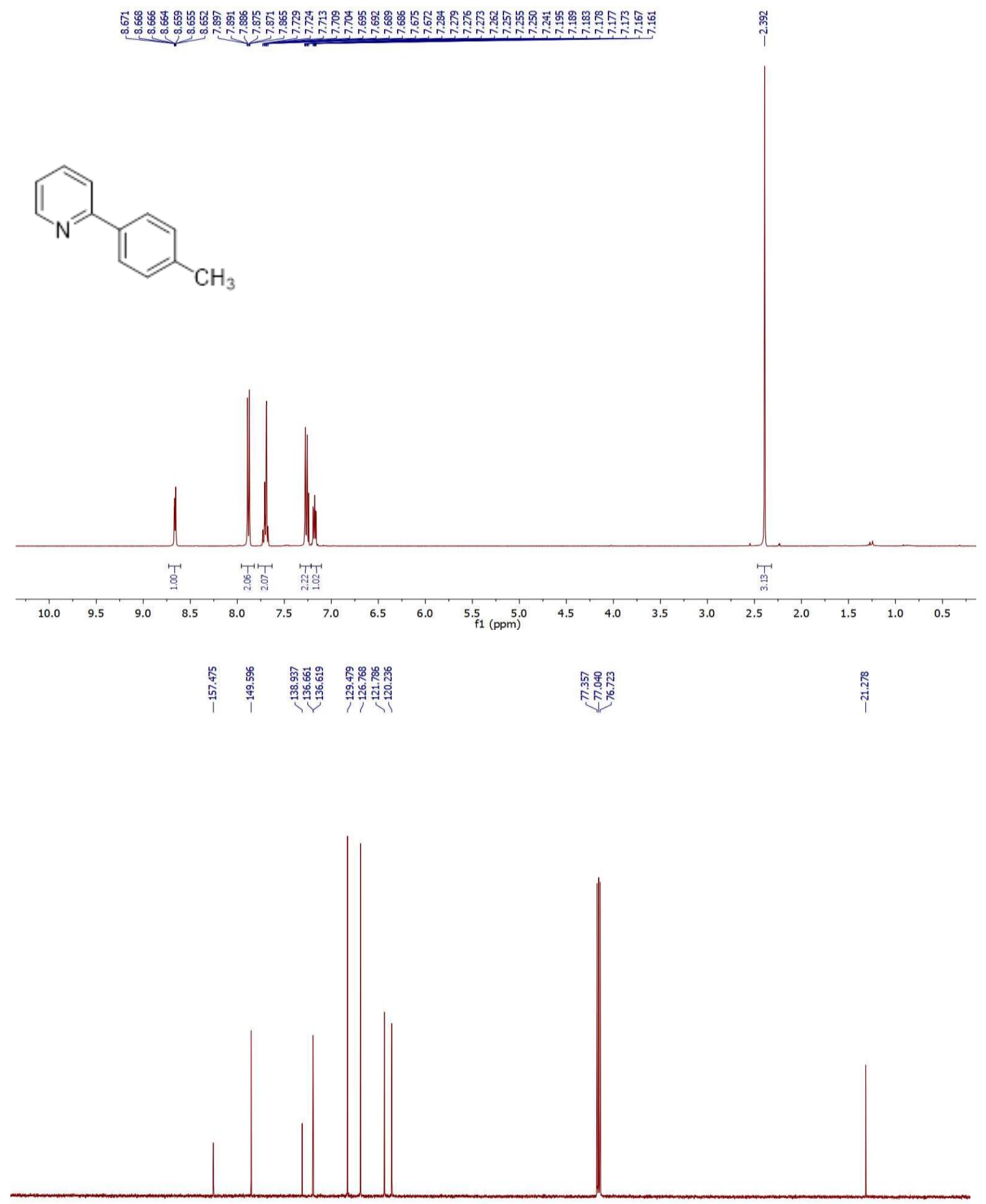
1-(4-Methylphenyl)-4-phenylpiperazine $19\left(\mathrm{CDCl}_{3}, 400 \mathrm{MHz}\right.$ for ${ }^{1} \mathrm{H} \mathrm{NMR}, 100 \mathrm{MHz}$ for $\left.{ }^{13} \mathrm{C} \mathrm{NMR}\right)$ nan9<smiles>Cc1ccc(N2CCN(c3ccccn3)CC2)cc1</smiles>

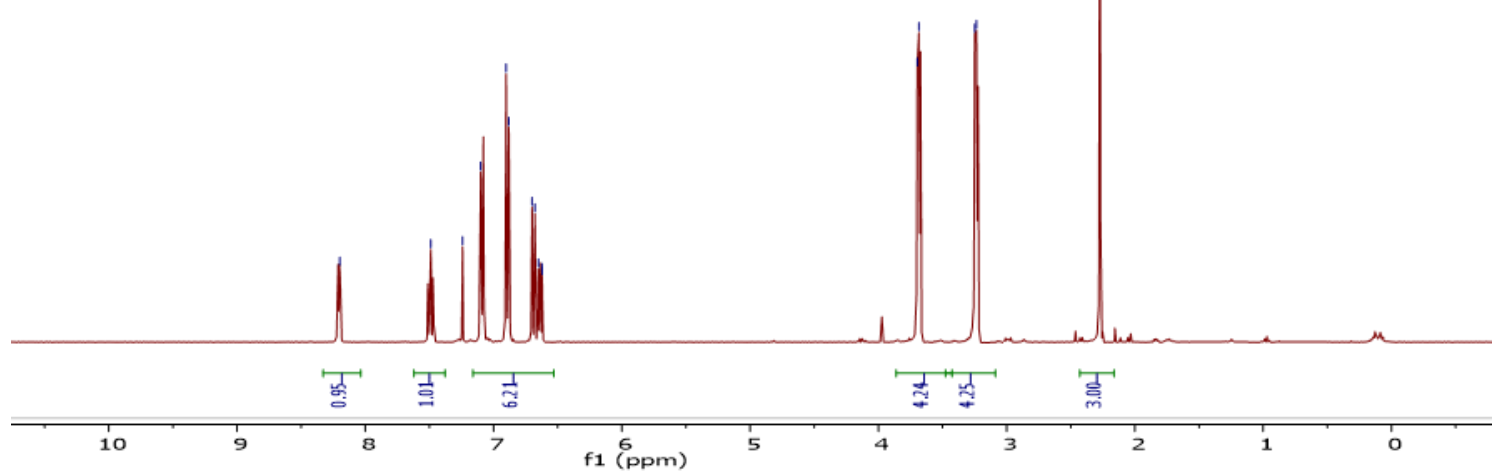

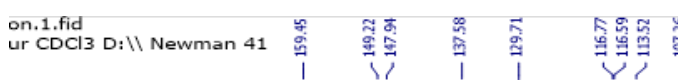

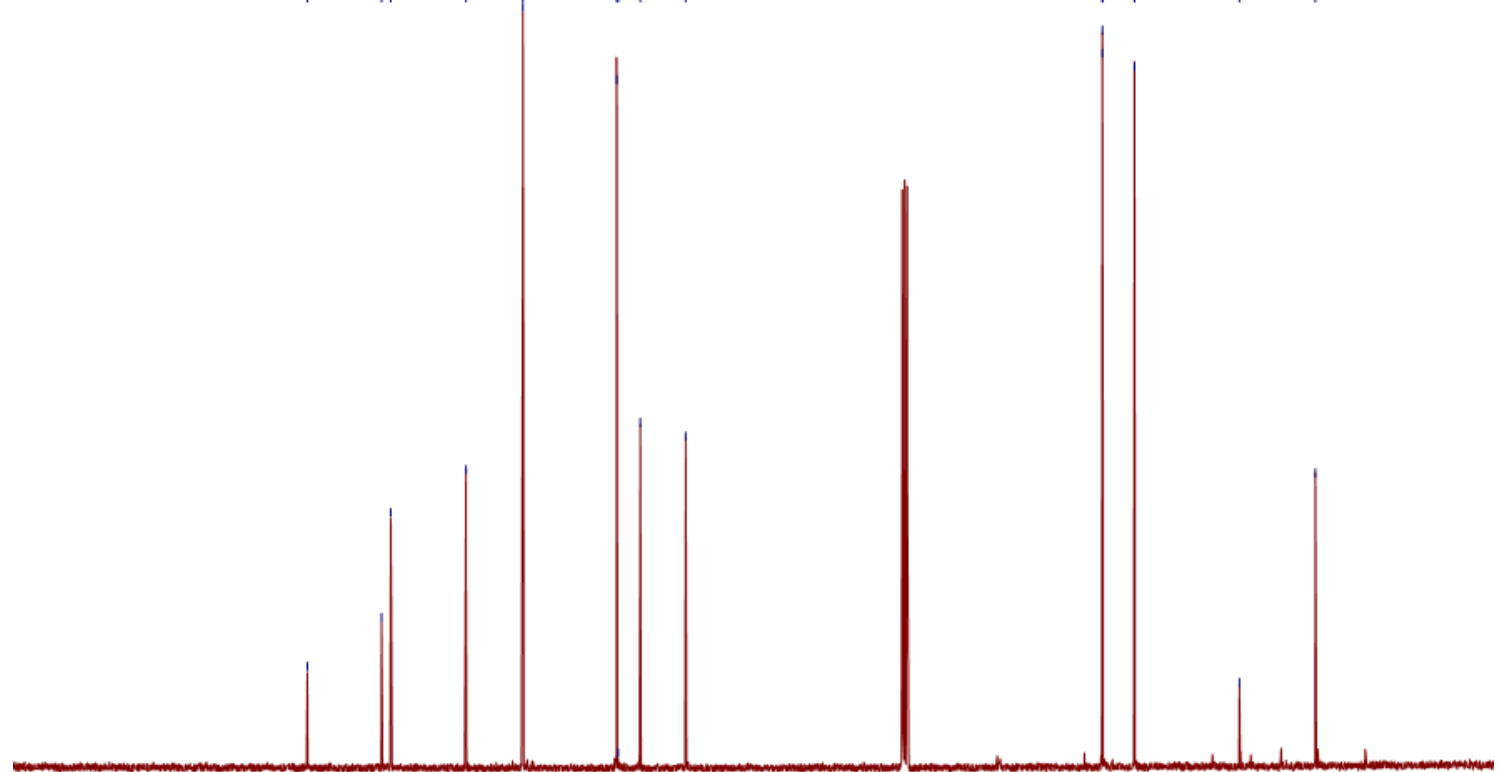

\begin{tabular}{lllllllllllllllllllllll}
\hline 1 & 190 & 180 & 170 & 160 & 150 & 140 & 130 & 120 & 110 & 100 & 90 & 80 & 70 & 60 & 50 & 40 & 30 & 20 & 10 & 0
\end{tabular} 
4-(5-Methyl-2-pyridinyl)-morpholine 20 ( $\mathrm{CDCl}_{3}, 400 \mathrm{MHz}$ for ${ }^{1} \mathrm{H} \mathrm{NMR}, 100 \mathrm{MHz}$ for ${ }^{13} \mathrm{C} \mathrm{NMR}$ )
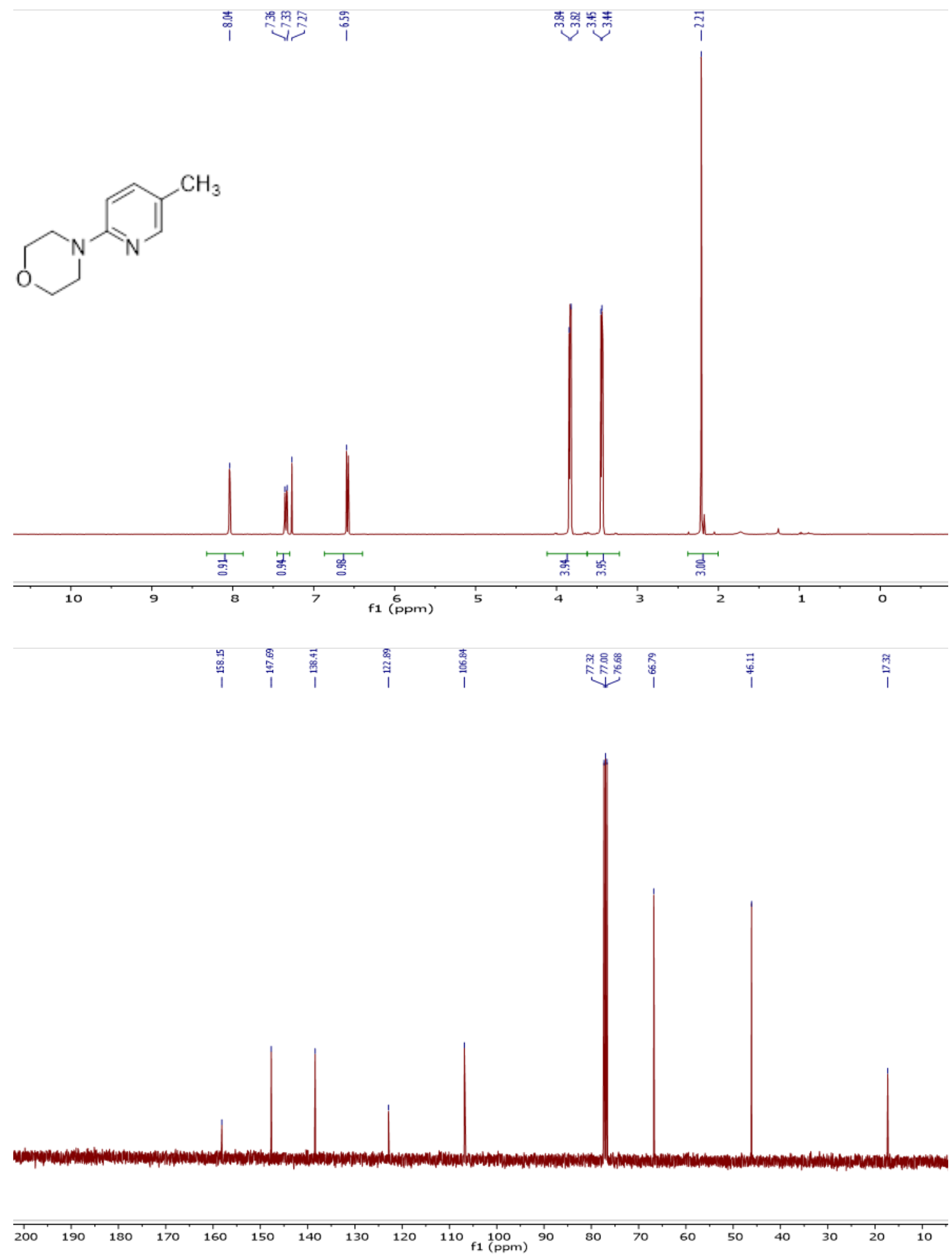
4-(2-Methylphenyl)-morpholine 21 ( $\mathrm{CDCl}_{3}, 400 \mathrm{MHz}$ for ${ }^{1} \mathrm{H} \mathrm{NMR}, 100 \mathrm{MHz}$ for ${ }^{13} \mathrm{C} \mathrm{NMR}$ )

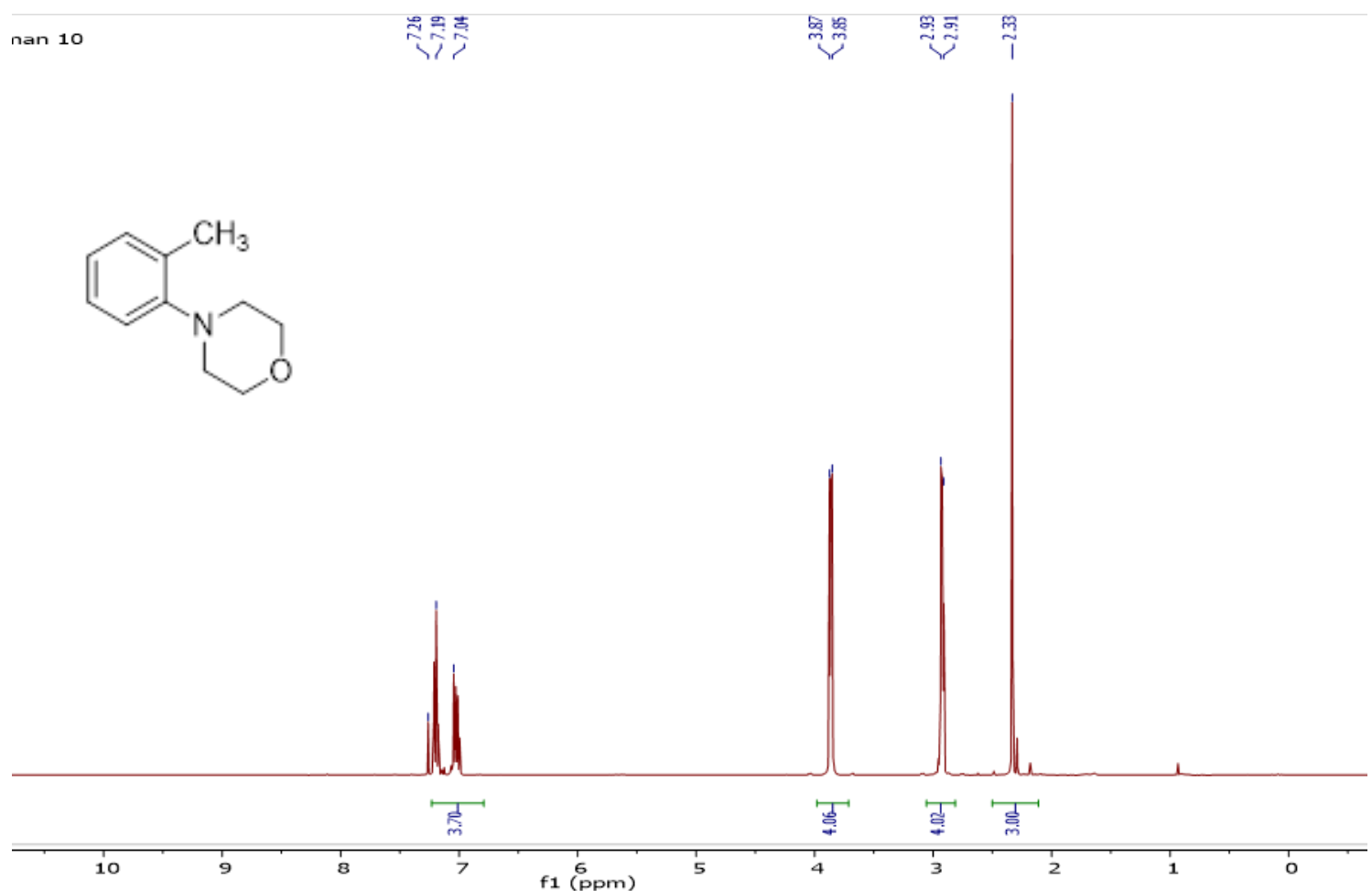

Jur CDC13 D:11 Newman重8

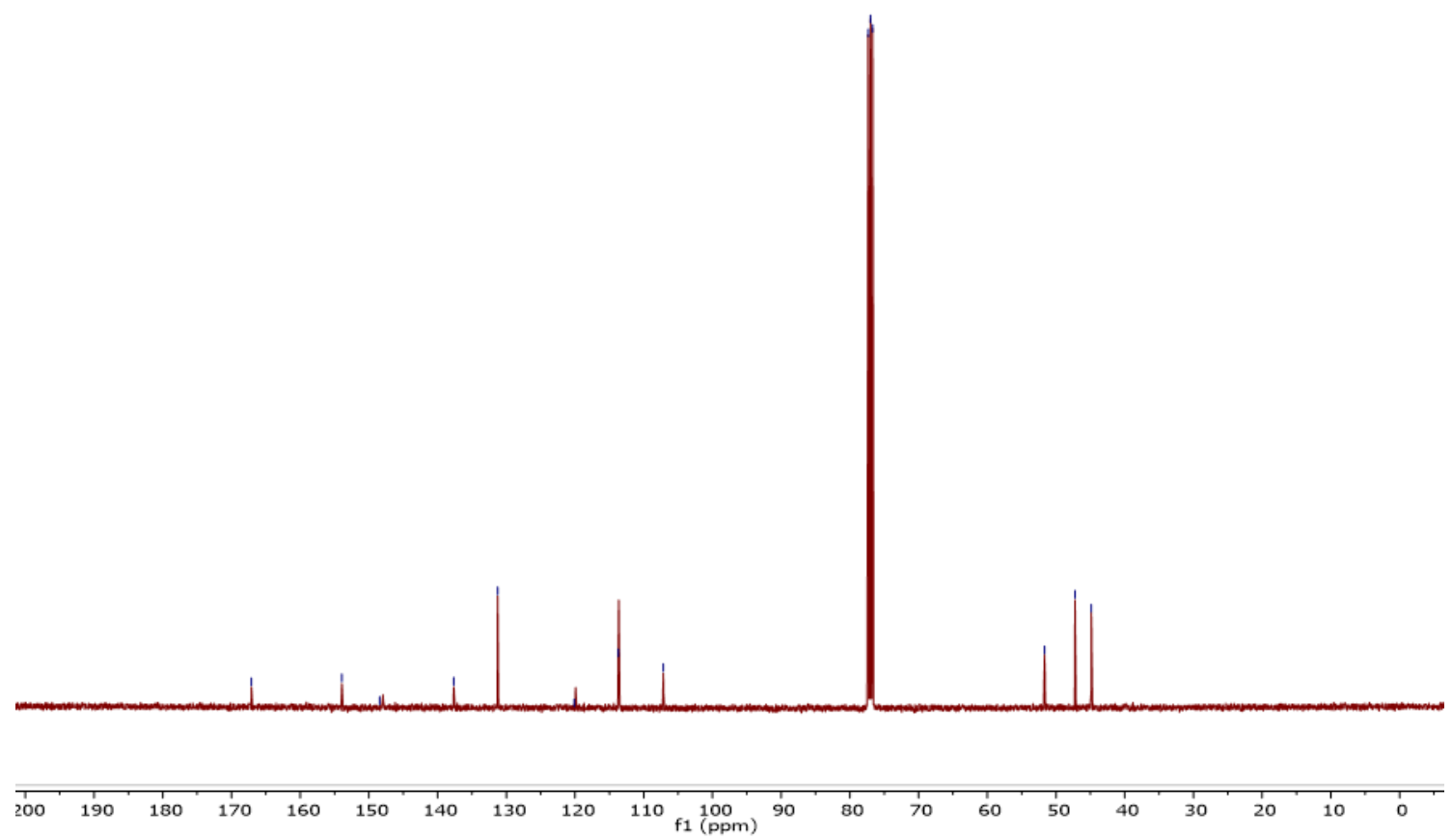


4-(p-Tolyl)morpholine $22\left(\mathrm{CDCl}_{3}, 400 \mathrm{MHz}\right.$ for ${ }^{1} \mathrm{H}$ NMR, $100 \mathrm{MHz}$ for $\left.{ }^{13} \mathrm{C} \mathrm{NMR}\right)$

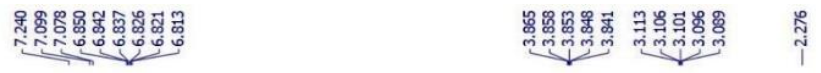<smiles>Cc1ccc(N2CCOCC2)cc1</smiles>
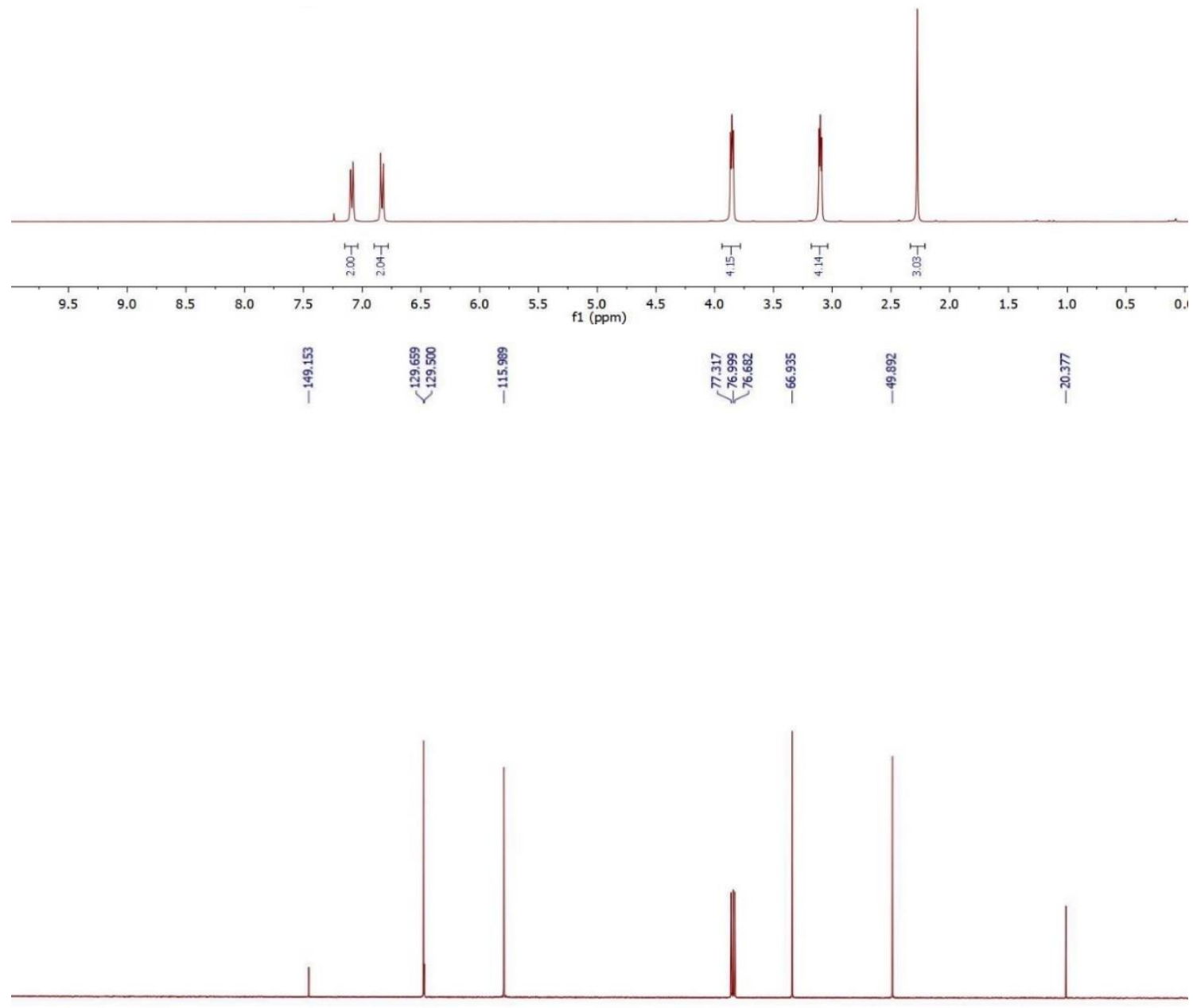
4-Methyl-1-(o-tolyl)piperidine 23 ( $\mathrm{CDCl}_{3}, 400 \mathrm{MHz}$ for ${ }^{1} \mathrm{H} \mathrm{NMR}, 100 \mathrm{MHz}$ for ${ }^{13} \mathrm{C} \mathrm{NMR}$ )

$\operatorname{nan} 47$
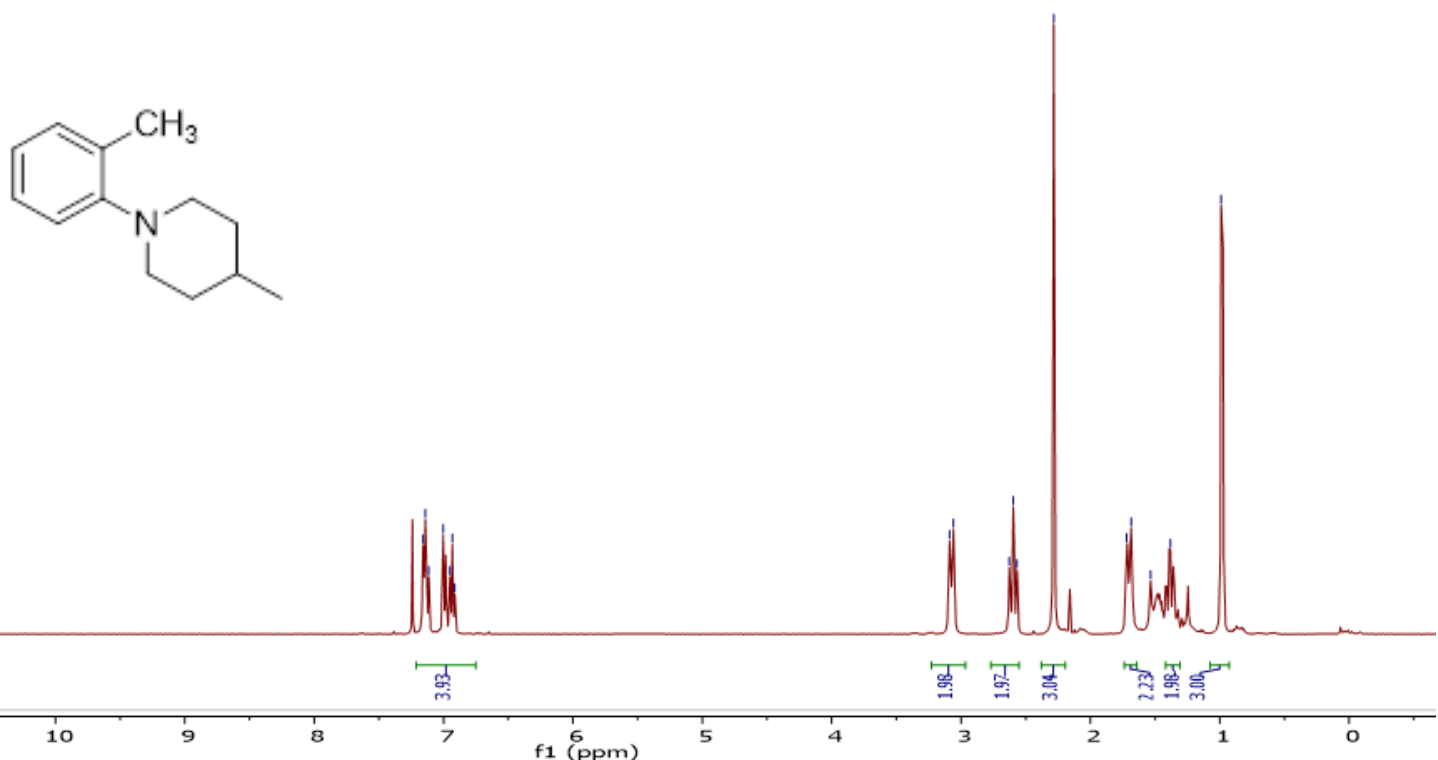

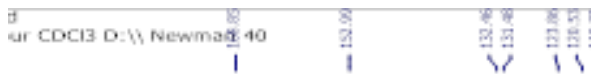
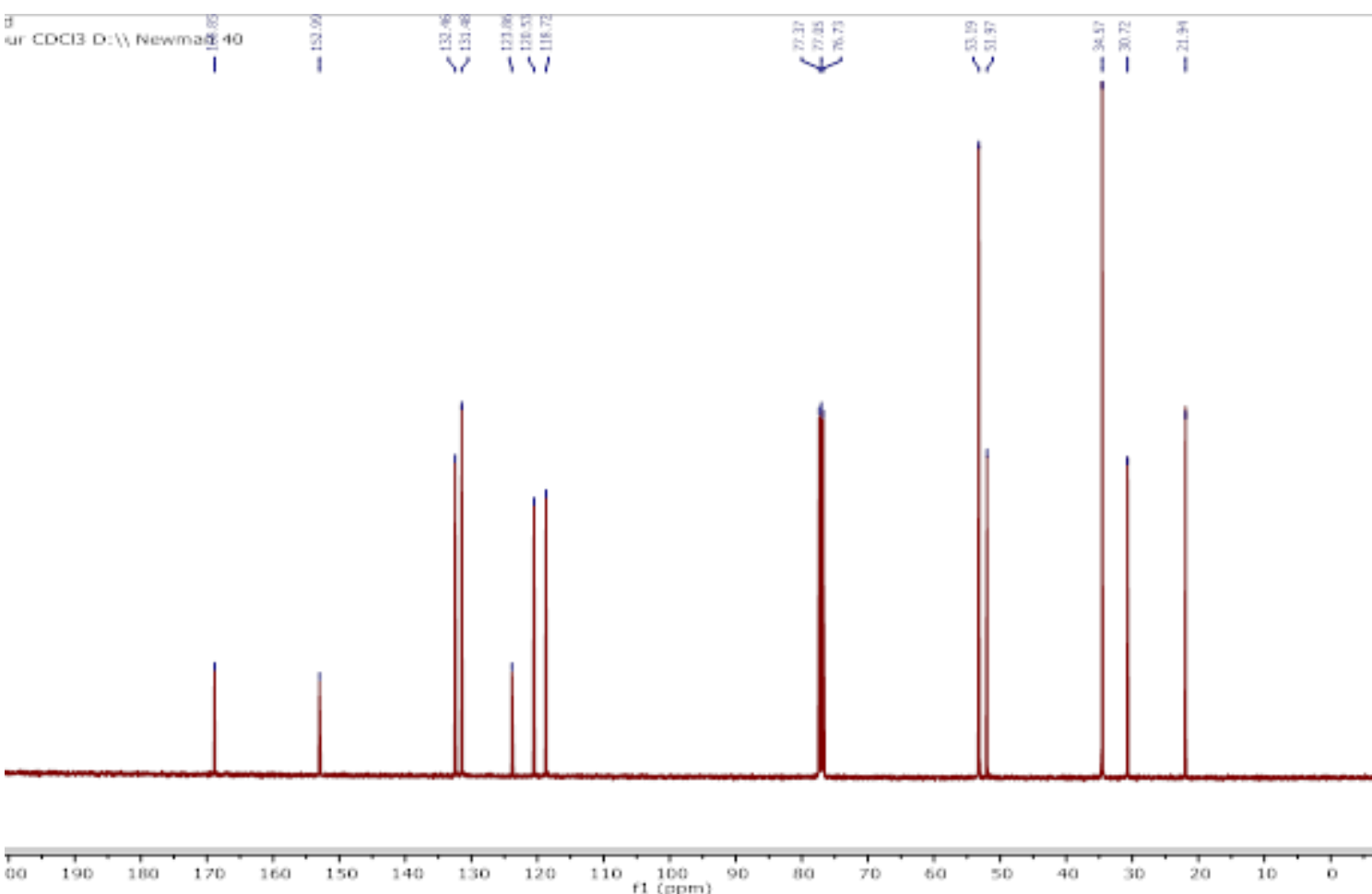
8-(4-Methylphenyl)-1,4-dioxa-8-azaspiro[4.5]decane $24\left(\mathrm{CDCl}_{3}, 400 \mathrm{MHz}\right.$ for ${ }^{1} \mathrm{H} \mathrm{NMR}, 100 \mathrm{MHz}$ for ${ }^{13} \mathrm{C}$ NMR)

$\operatorname{lan} 7$
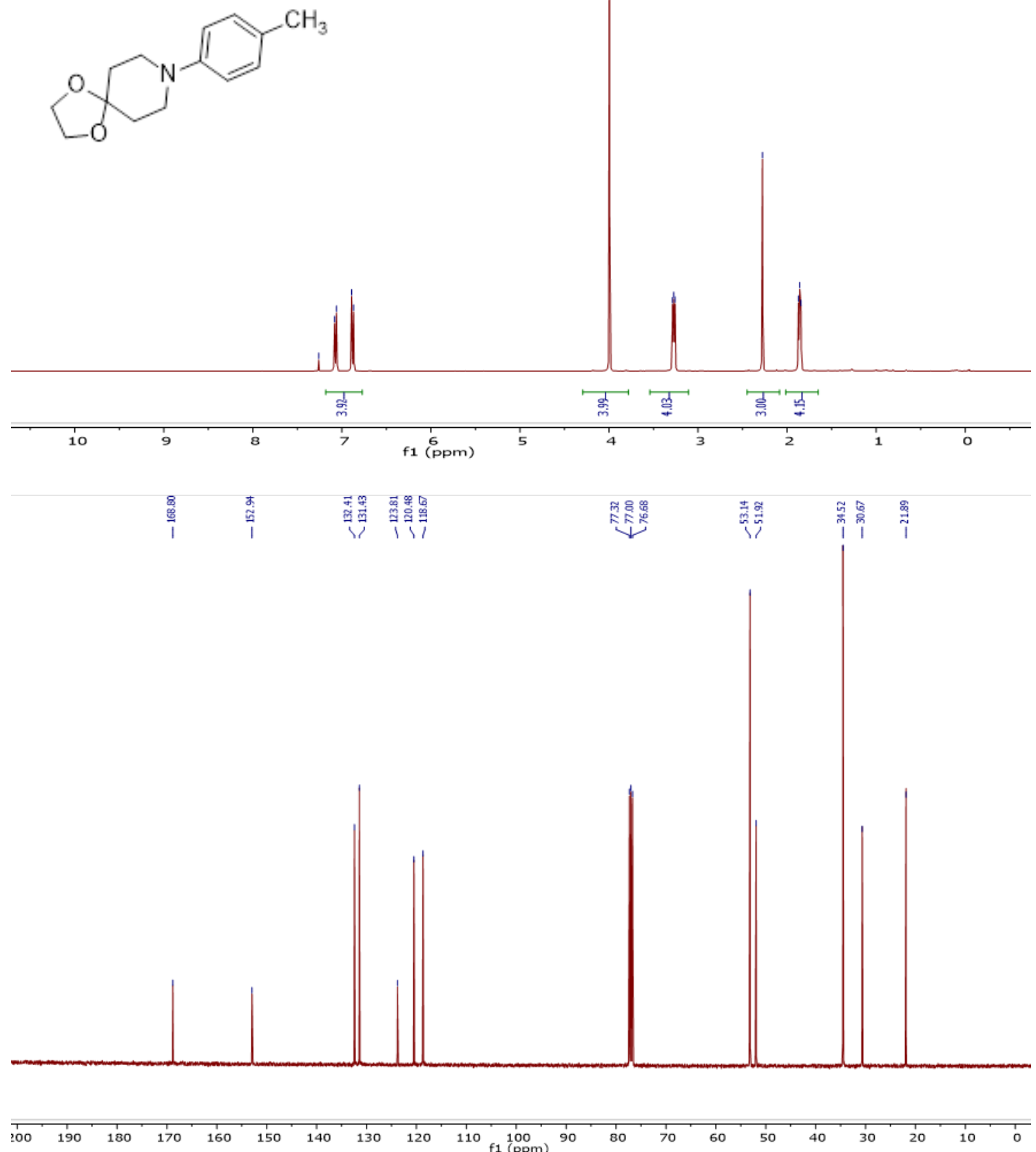
1,6-Dimethylindole $25\left(\mathrm{CDCl}_{3}, 400 \mathrm{MHz}\right.$ for ${ }^{1} \mathrm{H} \mathrm{NMR}, 100 \mathrm{MHz}$ for $\left.{ }^{13} \mathrm{C} \mathrm{NMR}\right)$

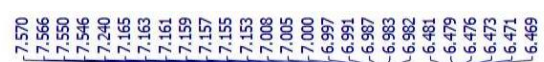
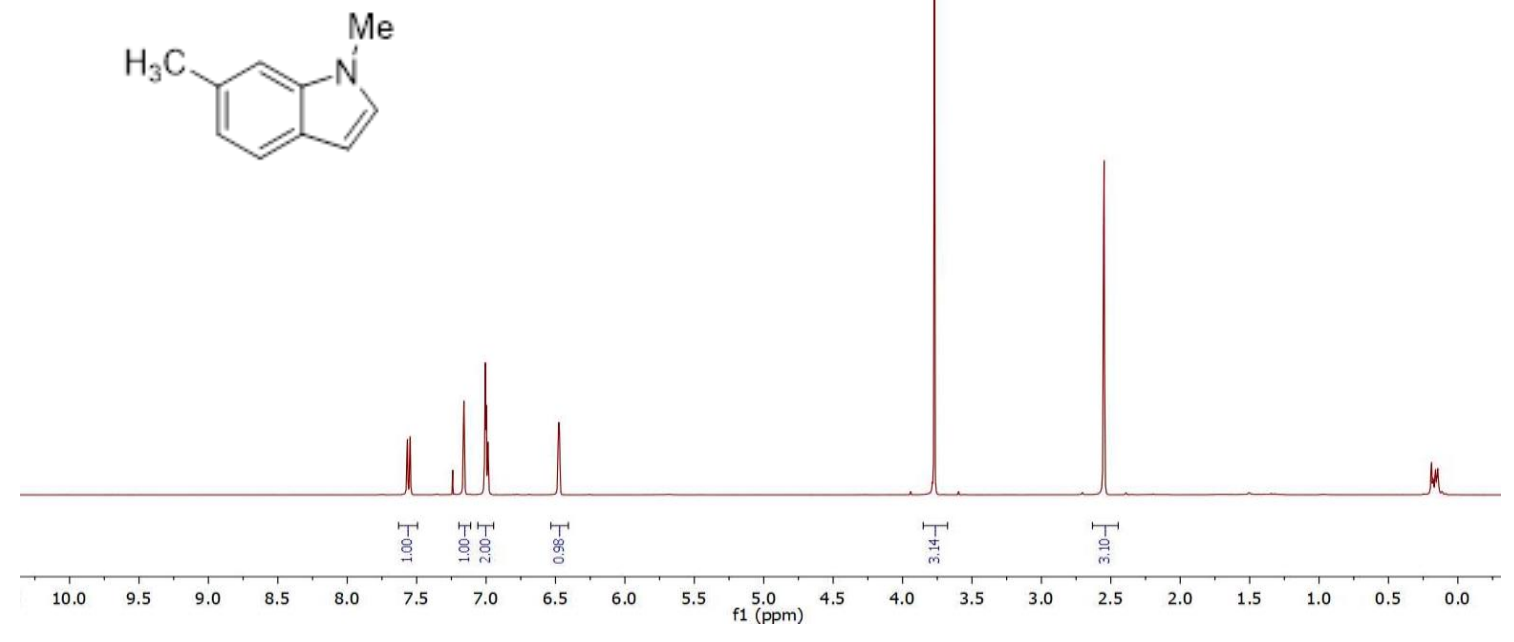

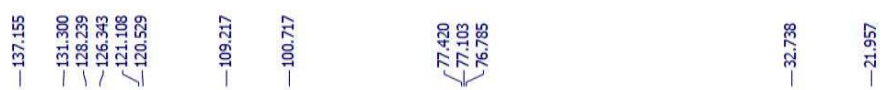

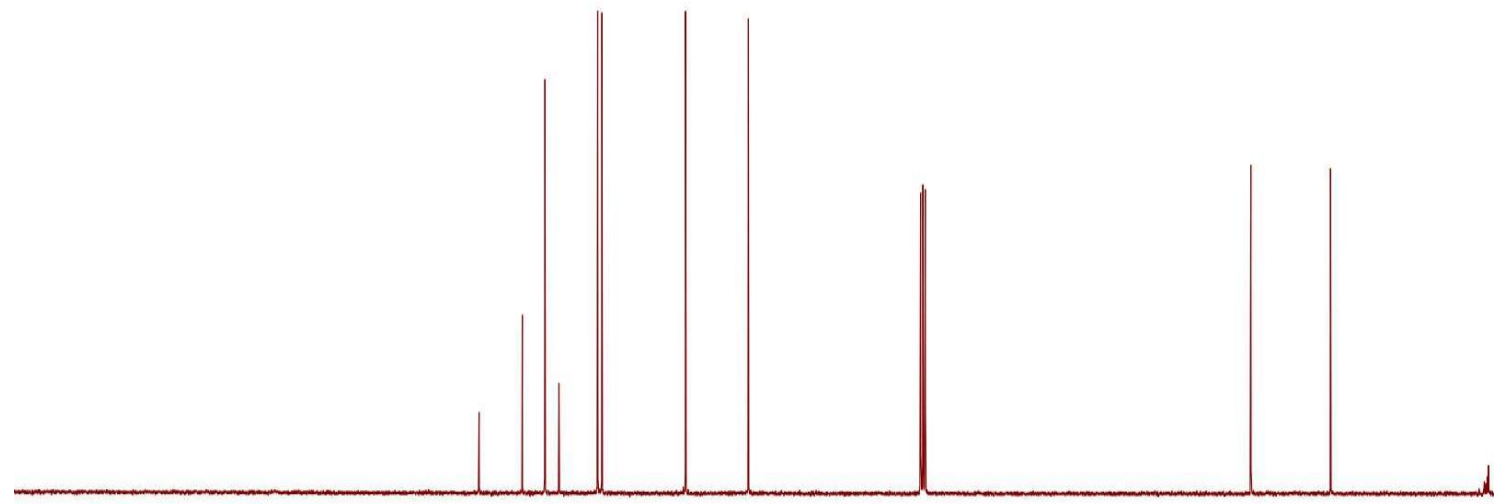


1,4-Dimethylindole $26\left(\mathrm{CDCl}_{3}, 400 \mathrm{MHz}\right.$ for ${ }^{1} \mathrm{H} \mathrm{NMR}, 100 \mathrm{MHz}$ for $\left.{ }^{13} \mathrm{C} \mathrm{NMR}\right)$

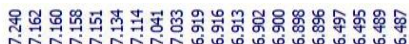

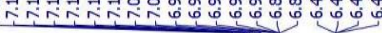

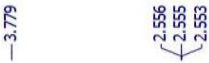<smiles>Cc1cccc2c1ccn2C</smiles>
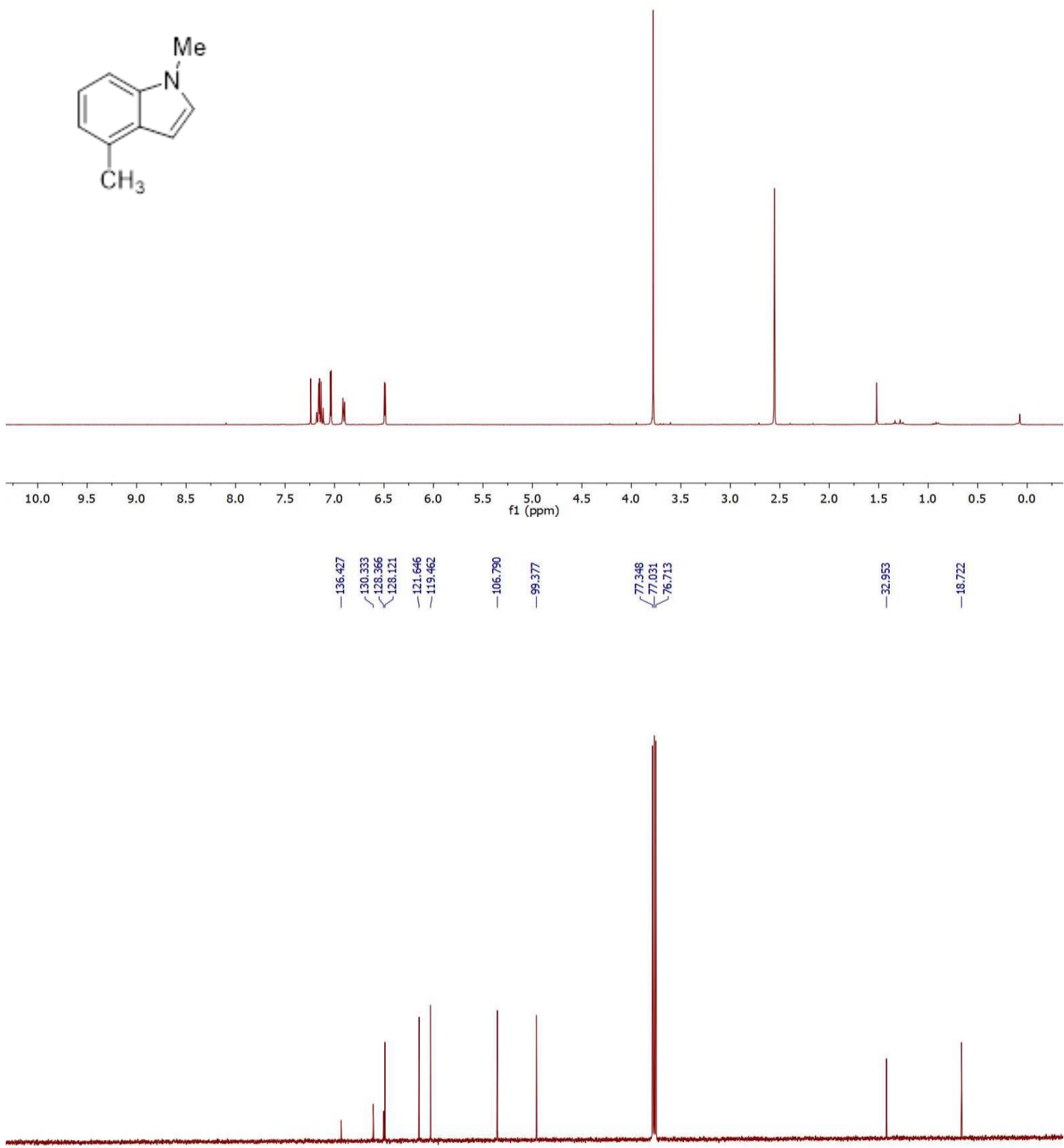

\begin{tabular}{llllllllllllllllllllll}
\hline 0 & 190 & 180 & 170 & 160 & 150 & 140 & 130 & 120 & 110 & 100 & 90 & 80 & 70 & 60 & 50 & 40 & 30 & 20 & 10 & 0
\end{tabular} 
1-(p-Tolyl)-indole $27\left(\mathrm{CDCl}_{3}, 400 \mathrm{MHz}\right.$ for ${ }^{1} \mathrm{H} \mathrm{NMR}, 100 \mathrm{MHz}$ for $\left.{ }^{13} \mathrm{C} \mathrm{NMR}\right)$

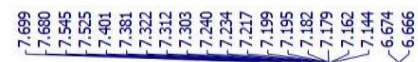

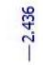
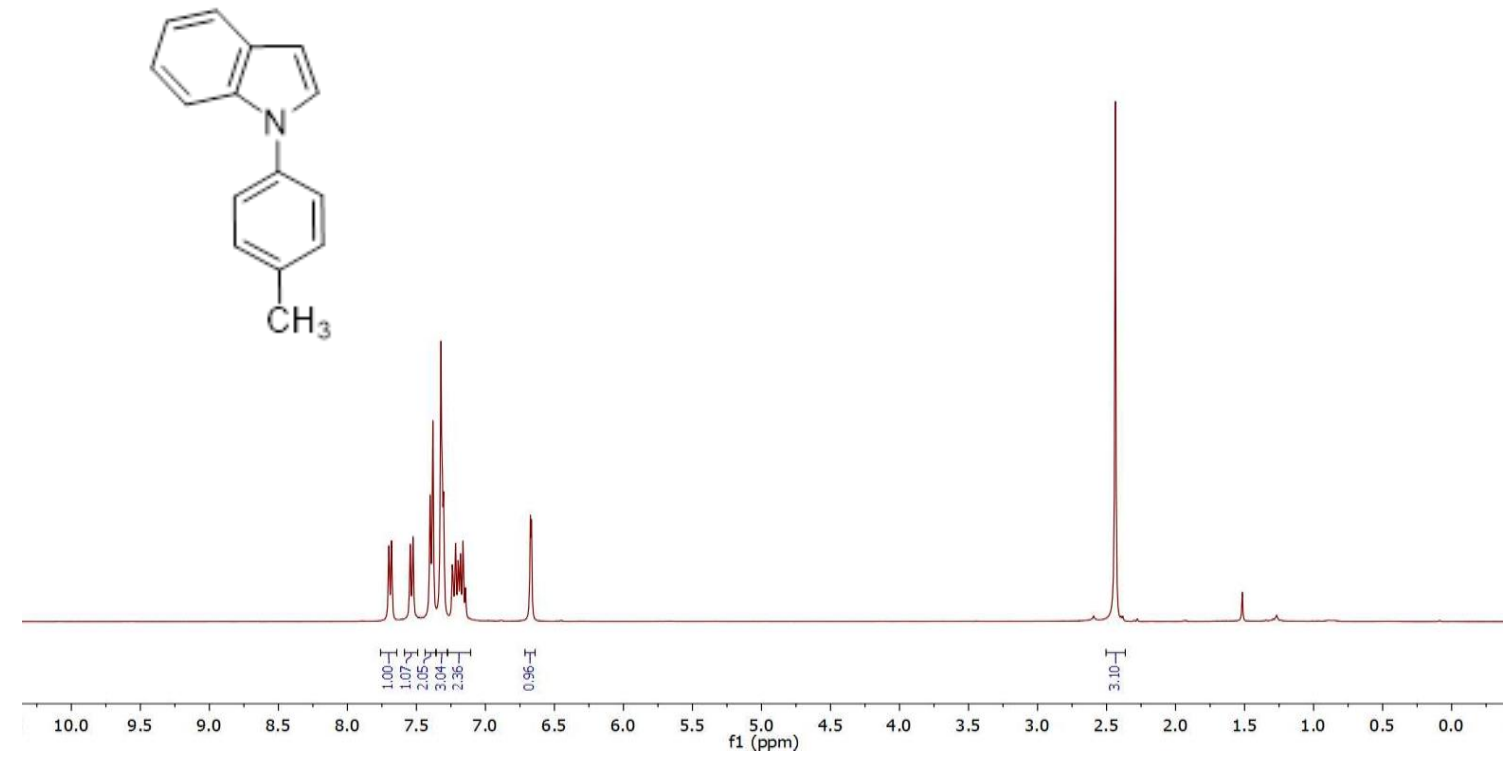

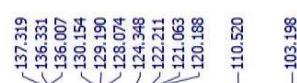
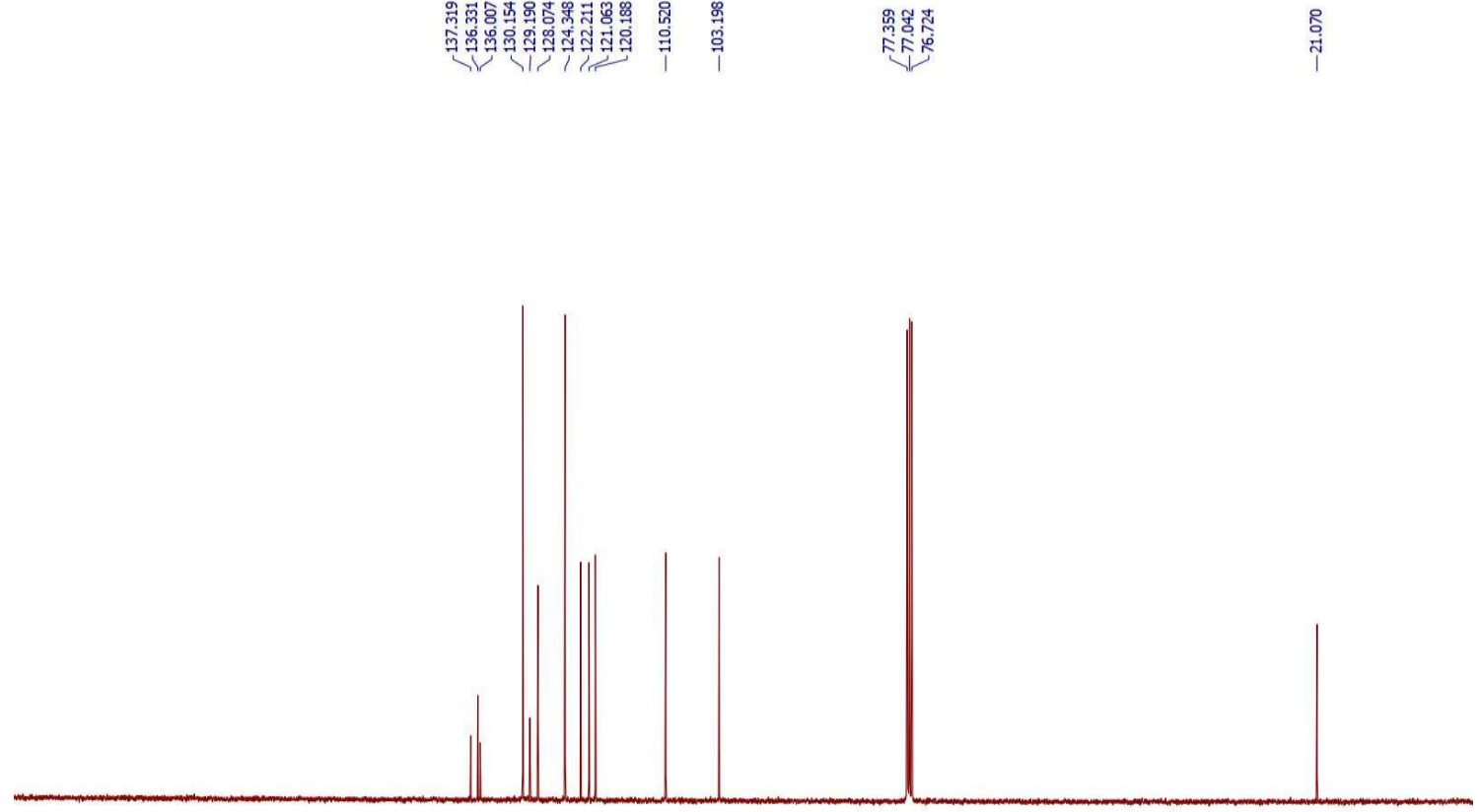
1-Methyl-3-hydroxy-4-isopropylbenzene $29\left(\mathrm{CDCl}_{3}, 400 \mathrm{MHz}\right.$ for ${ }^{1} \mathrm{H} \mathrm{NMR}, 100 \mathrm{MHz}$ for $\left.{ }^{13} \mathrm{C} \mathrm{NMR}\right)$

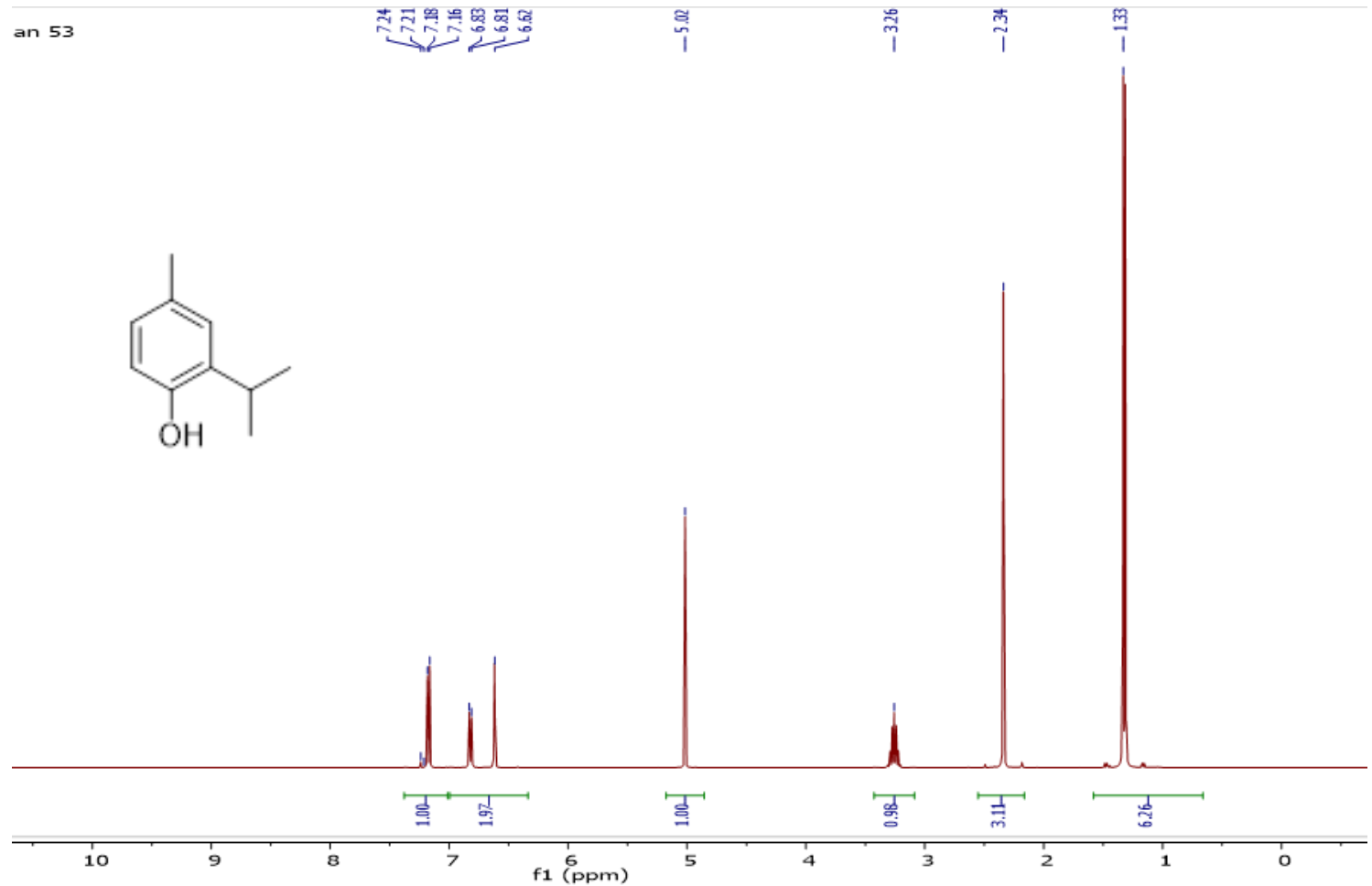

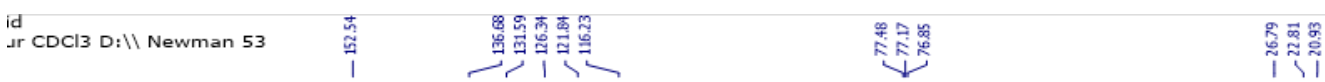

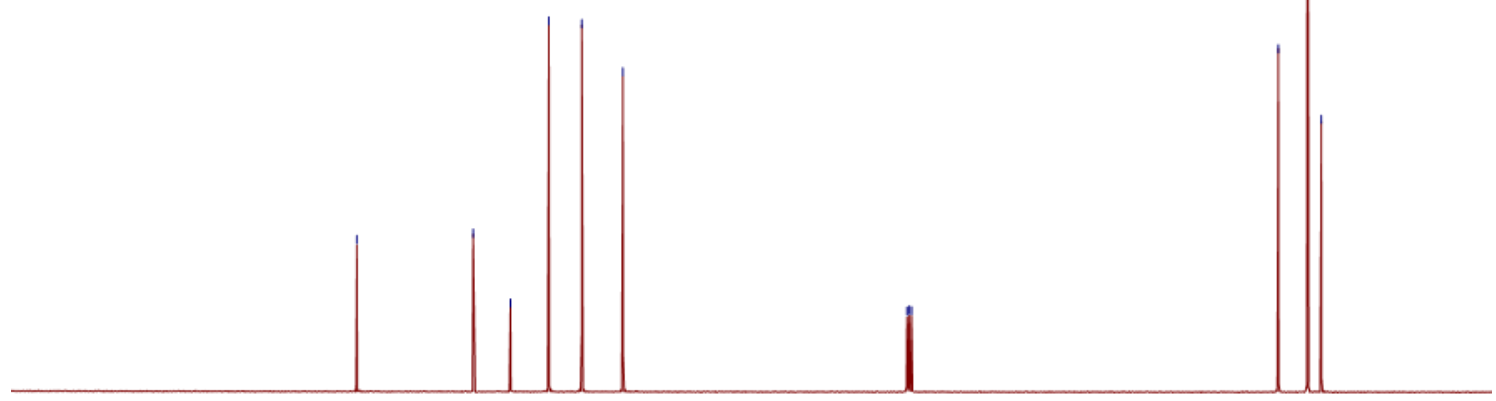

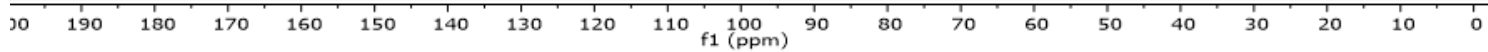


9-Benzyl-3-methylcarbazole $30\left(\mathrm{CDCl}_{3}, 400 \mathrm{MHz}\right.$ for ${ }^{1} \mathrm{H} \mathrm{NMR}, 100 \mathrm{MHz}$ for $\left.{ }^{13} \mathrm{C} \mathrm{NMR}\right)$

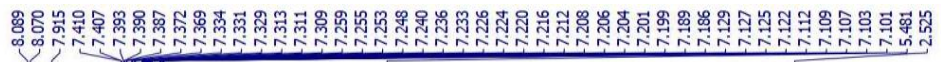

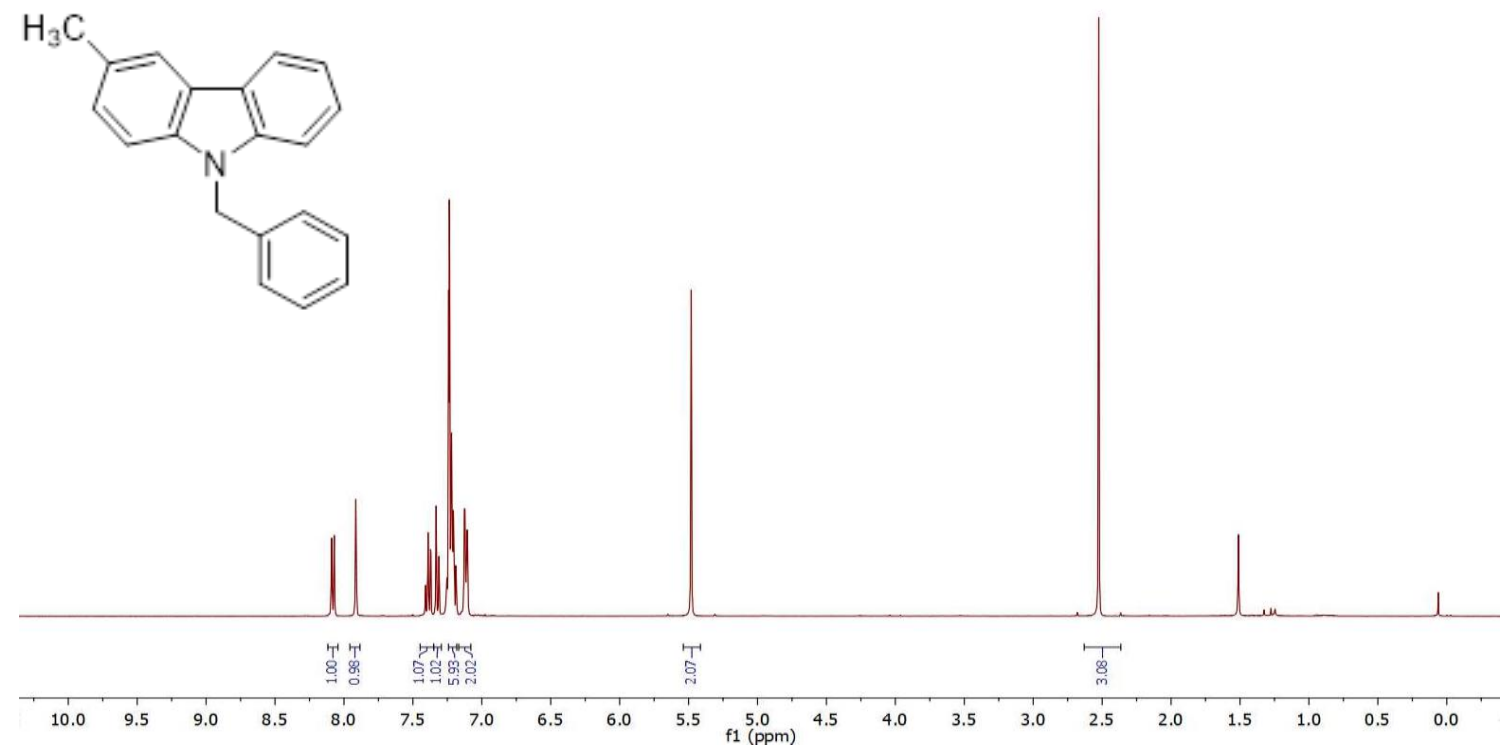

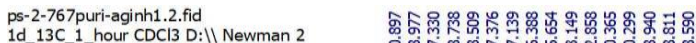

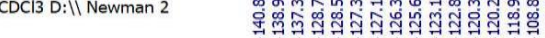
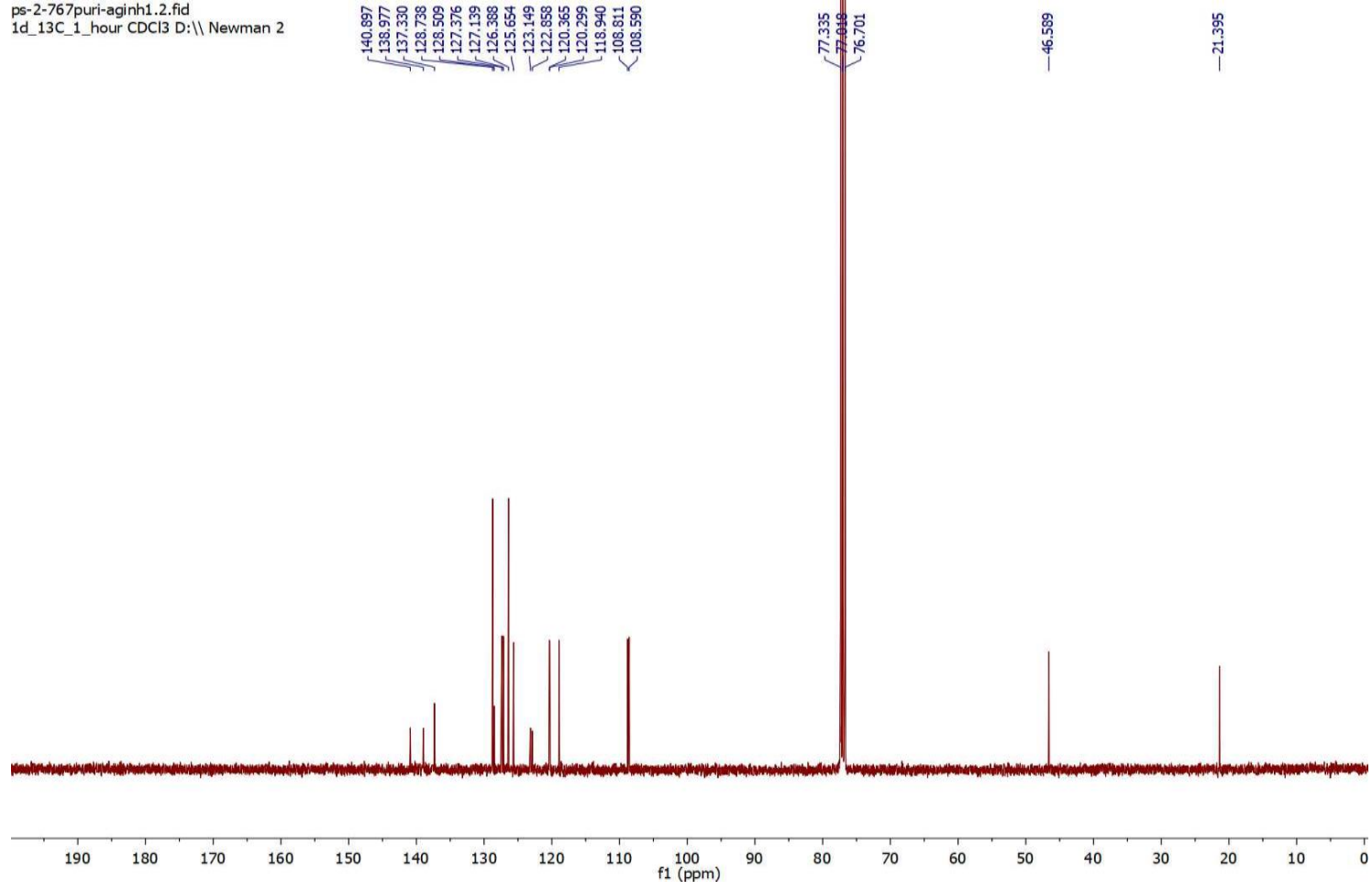
3-Methoxy-6,9-dimethyl-9-carbazole 31 ( $\mathrm{CDCl}_{3}, 400 \mathrm{MHz}$ for ${ }^{1} \mathrm{H} \mathrm{NMR}, 100 \mathrm{MHz}$ for ${ }^{13} \mathrm{C} \mathrm{NMR}$ )
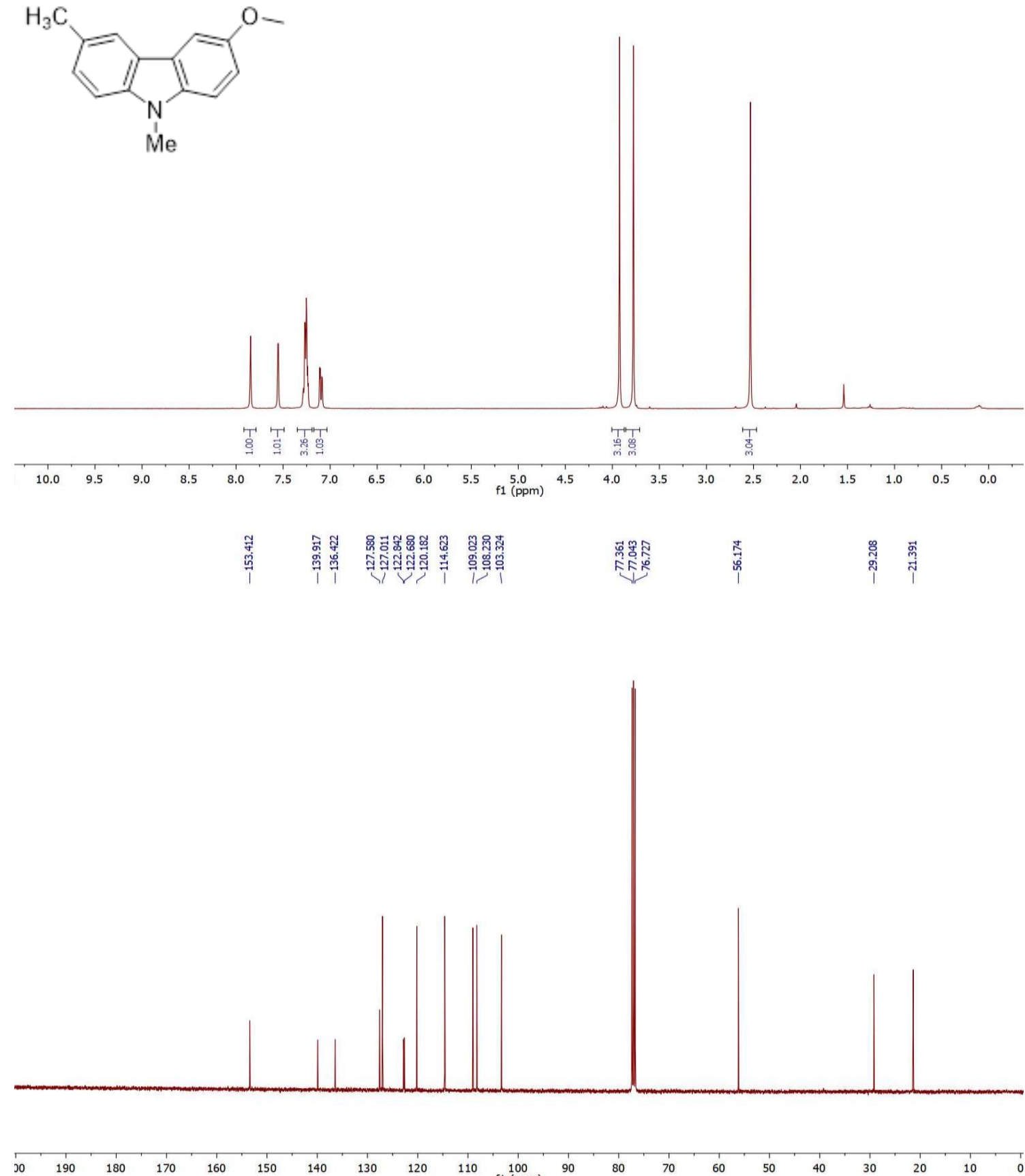
3-(t-Butyl)-6,9-dimethyl-carbazole $32\left(\mathrm{CDCl}_{3}, 400 \mathrm{MHz}\right.$ for ${ }^{1} \mathrm{H} \mathrm{NMR}, 100 \mathrm{MHz}$ for $\left.{ }^{13} \mathrm{C} \mathrm{NMR}\right)$

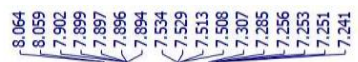

量
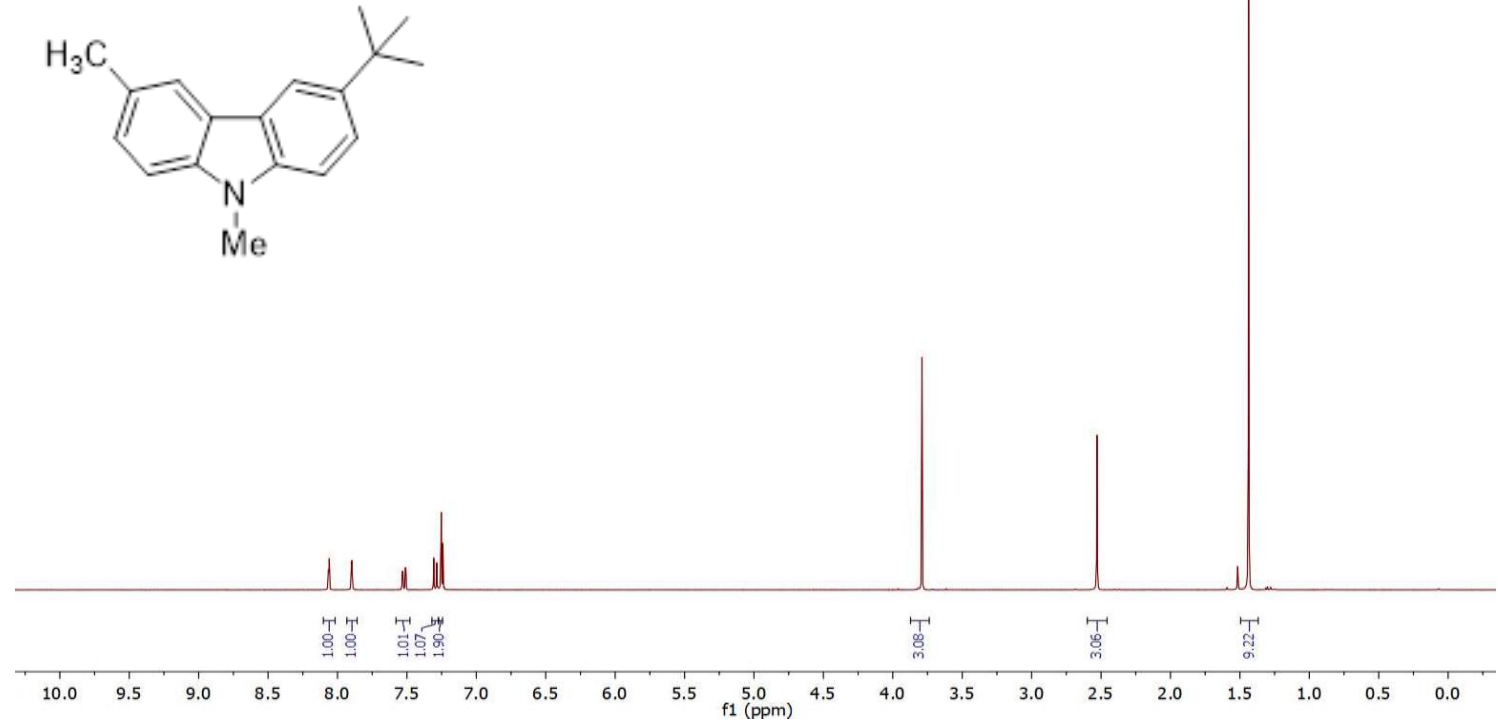

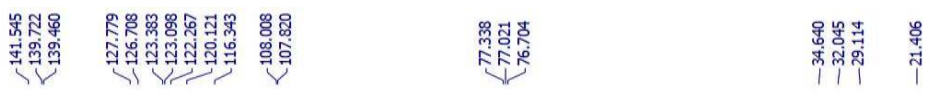

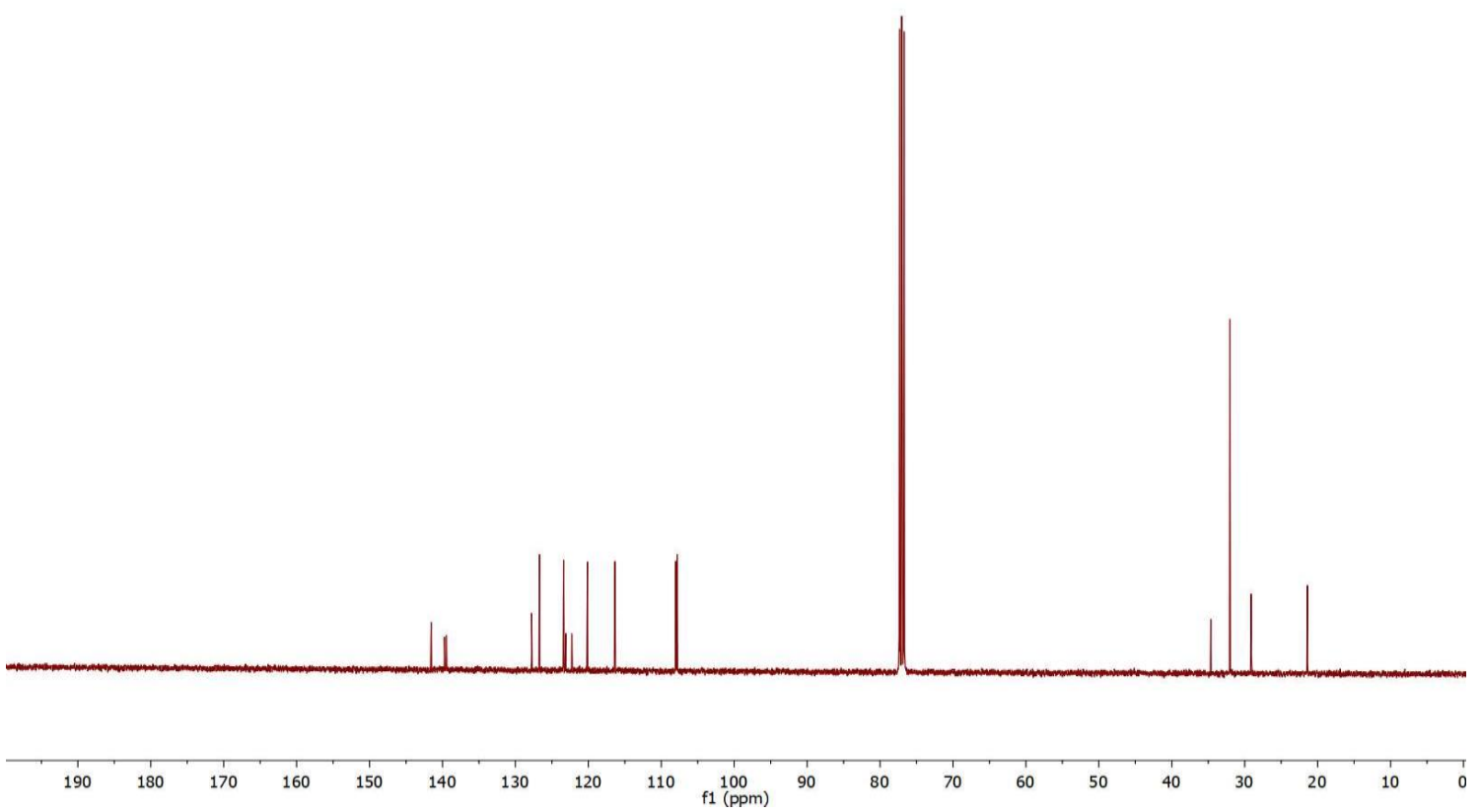


1,3-Dimethylindazole $33\left(\mathrm{CDCl}_{3}, 400 \mathrm{MHz}\right.$ for ${ }^{1} \mathrm{H} \mathrm{NMR}, 100 \mathrm{MHz}$ for ${ }^{13} \mathrm{C} \mathrm{NMR}$ )
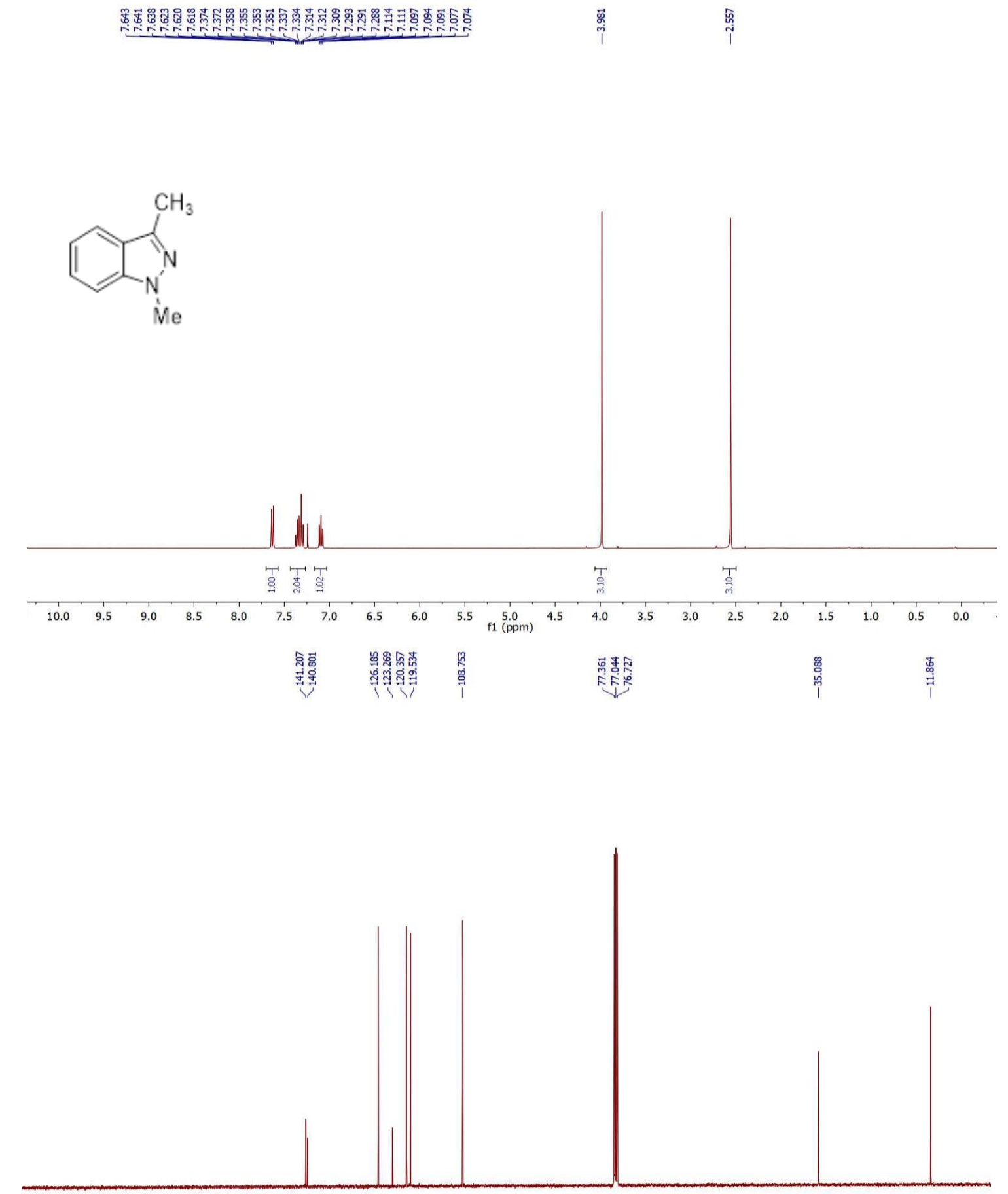
6-Methylquinoline $34\left(\mathrm{CDCl}_{3}, 400 \mathrm{MHz}\right.$ for ${ }^{1} \mathrm{H} \mathrm{NMR}, 100 \mathrm{MHz}$ for $\left.{ }^{13} \mathrm{C} \mathrm{NMR}\right)$
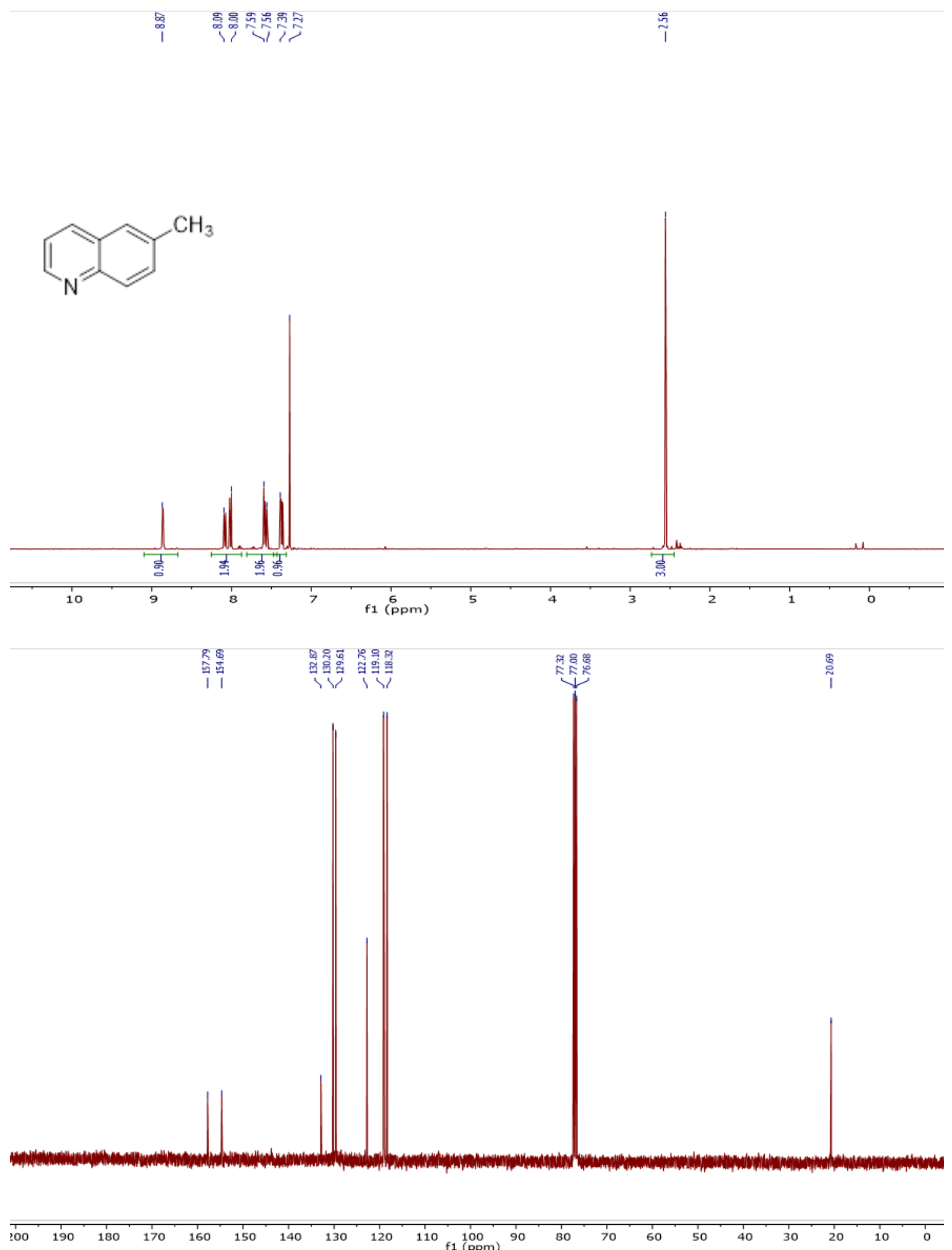
7-Methoxy-2-methylbenzofuran $35\left(\mathrm{CDCl}_{3}, 400 \mathrm{MHz}\right.$ for ${ }^{1} \mathrm{H} \mathrm{NMR}, 100 \mathrm{MHz}$ for $\left.{ }^{13} \mathrm{C} \mathrm{NMR}\right)$

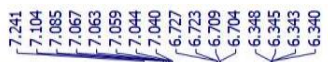

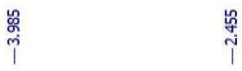
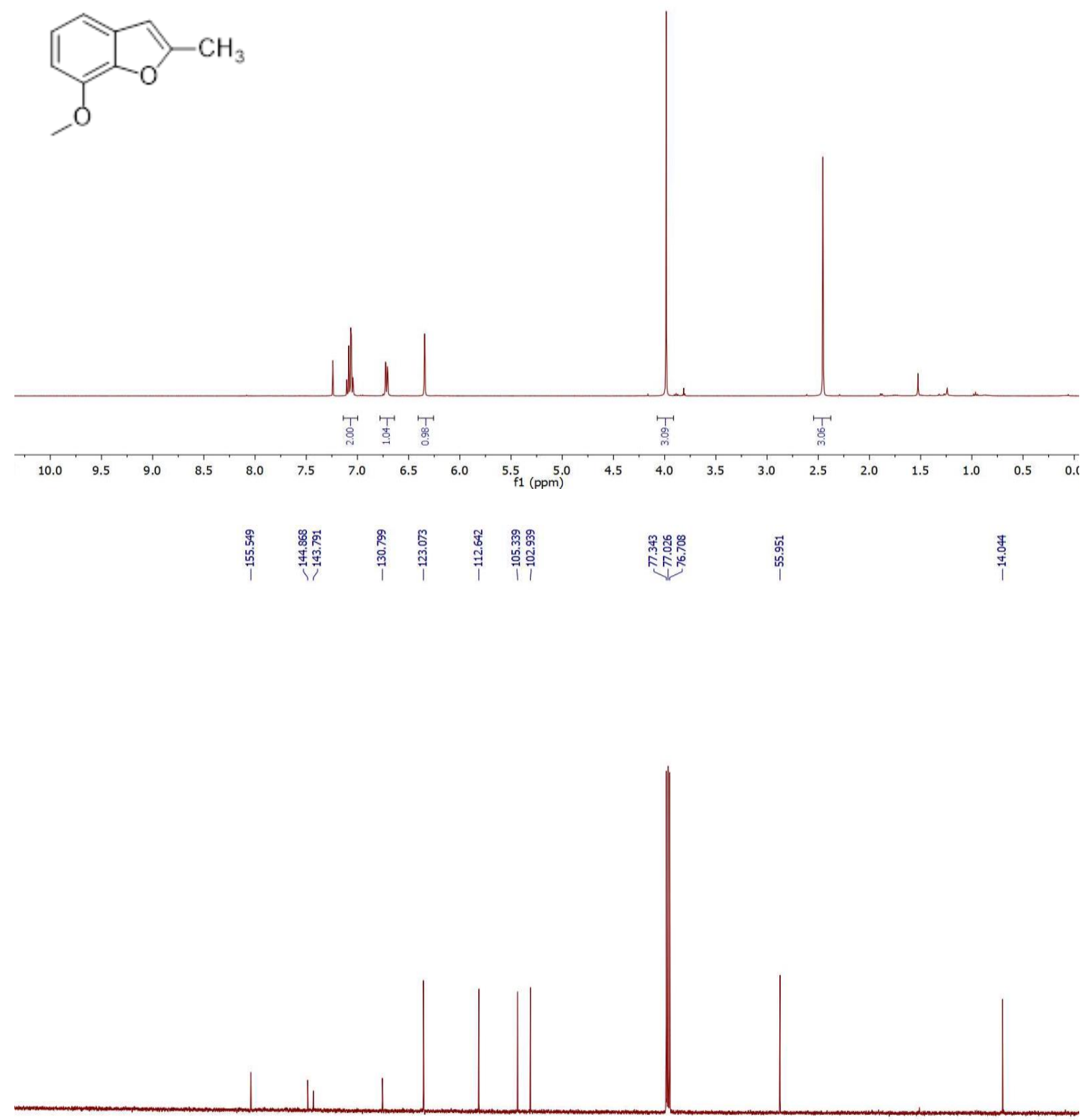
2-Methoxy-8-methyl-dibenzofuran $36\left(\mathrm{CDCl}_{3}, 400 \mathrm{MHz}\right.$ for ${ }^{1} \mathrm{H} \mathrm{NMR}, 100 \mathrm{MHz}$ for $\left.{ }^{13} \mathrm{C} \mathrm{NMR}\right)$
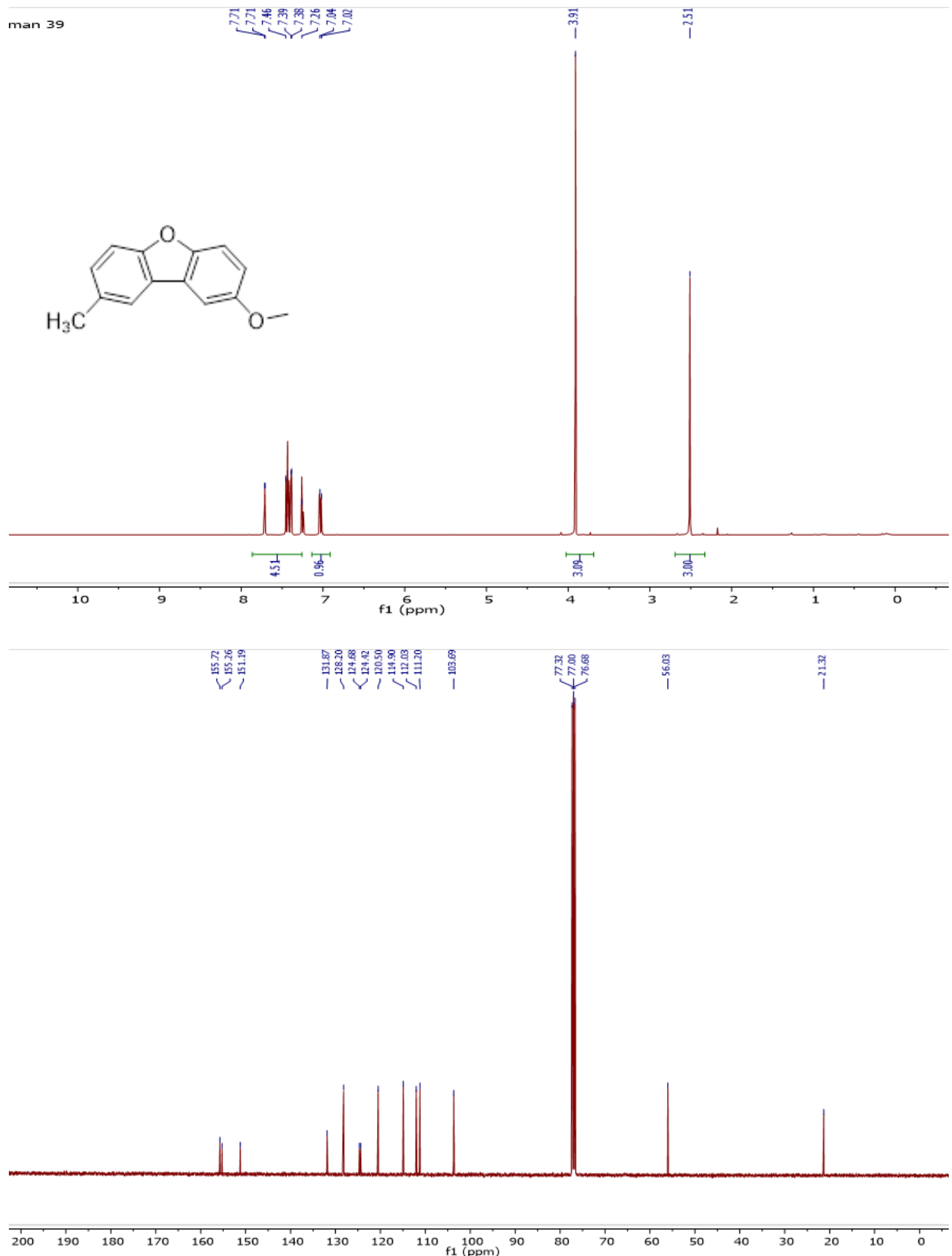
7,7'-Dimethoxy-5,5'-dimethyl-4,4'-bibenzo[d][1,3]dioxole $37\left(\mathrm{CDCl}_{3}, 400 \mathrm{MHz}\right.$ for ${ }^{1} \mathrm{H} \mathrm{NMR}, 100 \mathrm{MHz}$ for ${ }^{13}$ ( NMR)
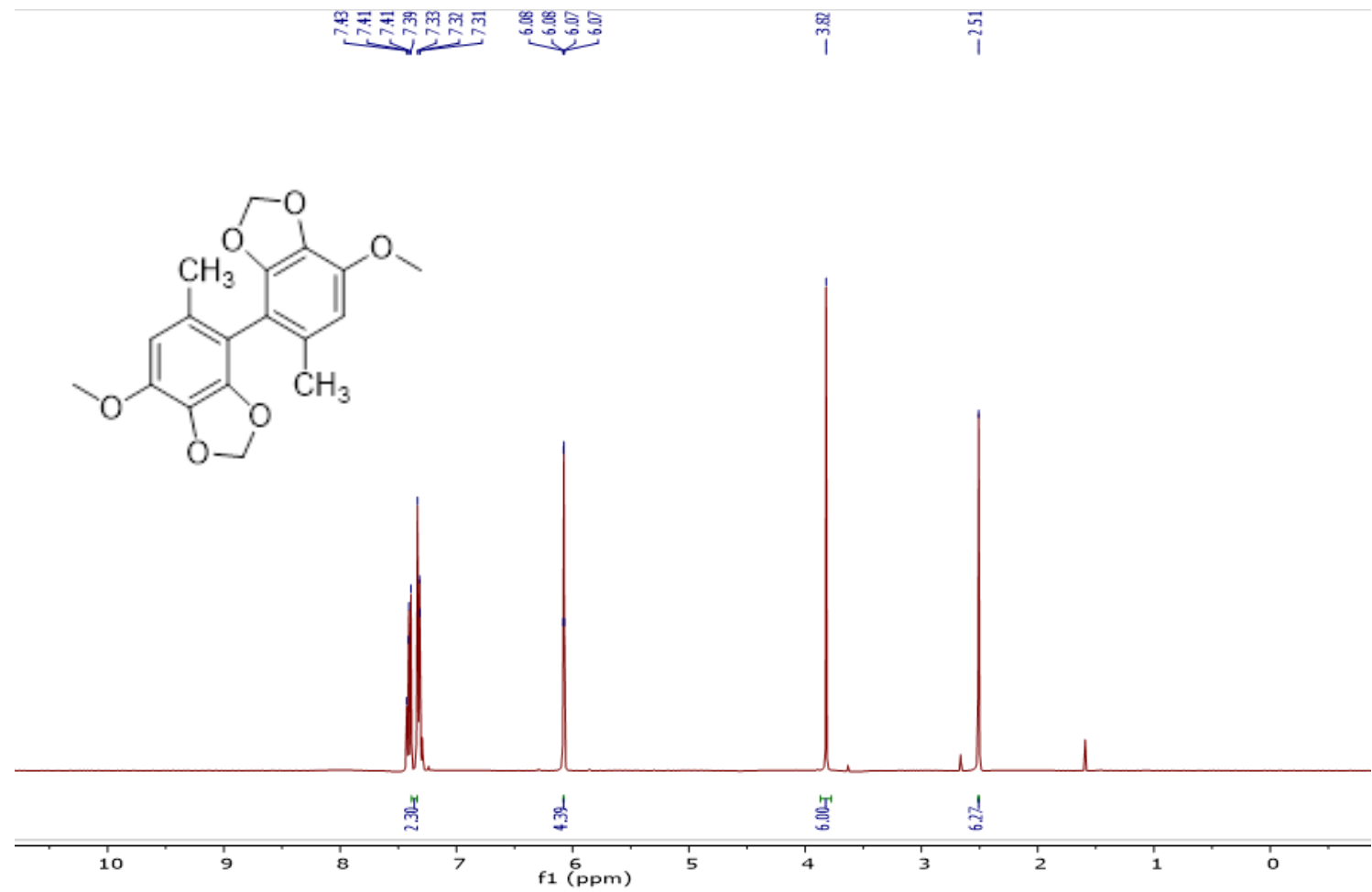

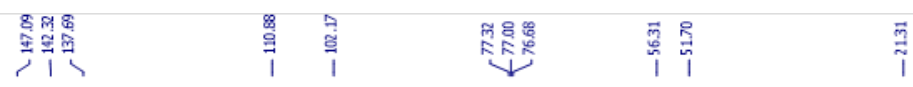
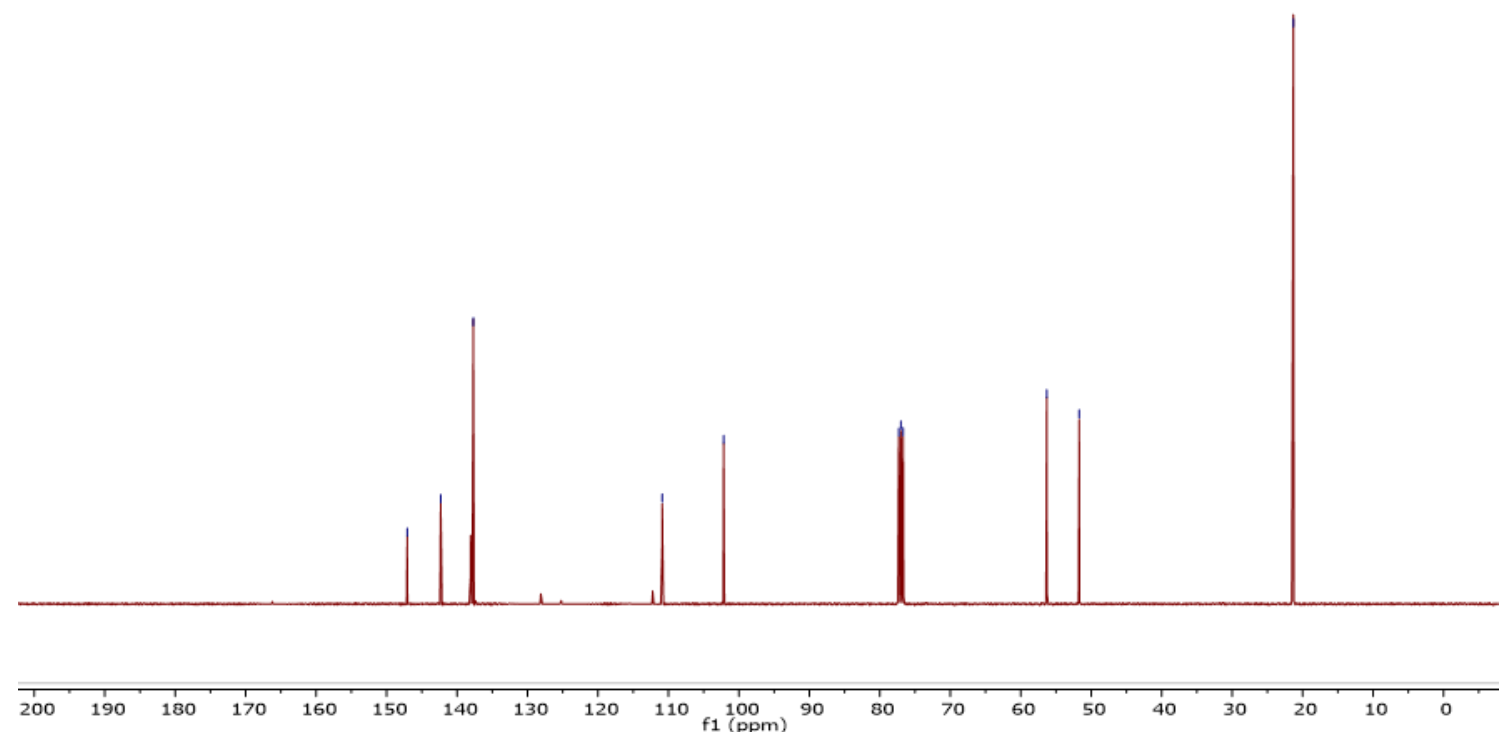
4-Methyl- $N, N$-dipropylbenzenesulfonamide $38\left(\mathrm{CDCl}_{3}, 400 \mathrm{MHz}\right.$ for ${ }^{1} \mathrm{H} \mathrm{NMR}, 100 \mathrm{MHz}$ for $\left.{ }^{13} \mathrm{C} \mathrm{NMR}\right)$
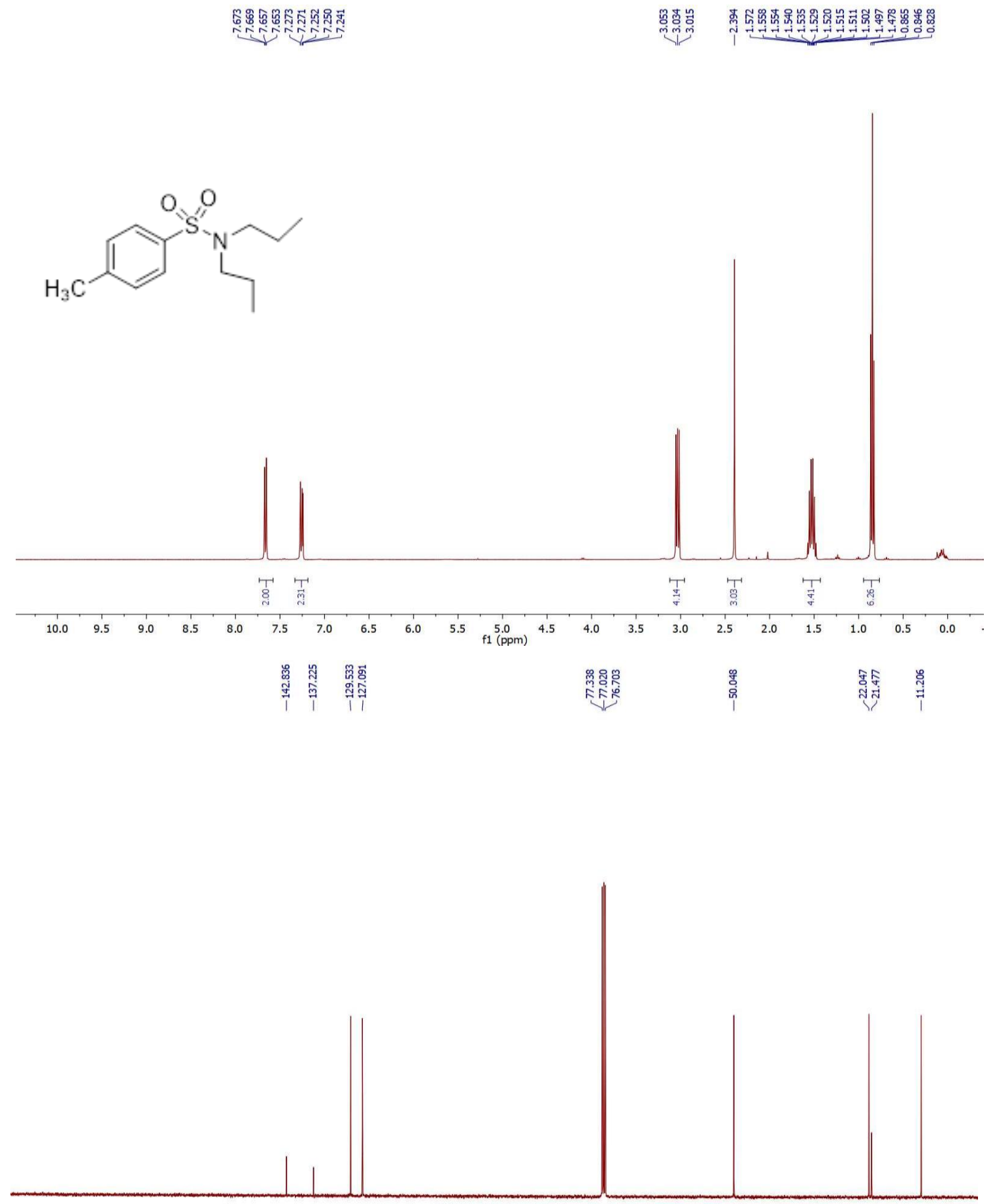

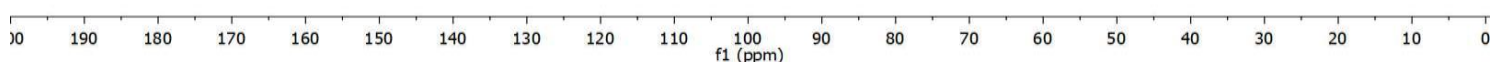


2-(Methylbenzene)ethanol $39\left(\mathrm{CDCl}_{3}, 400 \mathrm{MHz}\right.$ for ${ }^{1} \mathrm{H} \mathrm{NMR}, 100 \mathrm{MHz}$ for $\left.{ }^{13} \mathrm{C} \mathrm{NMR}\right)$

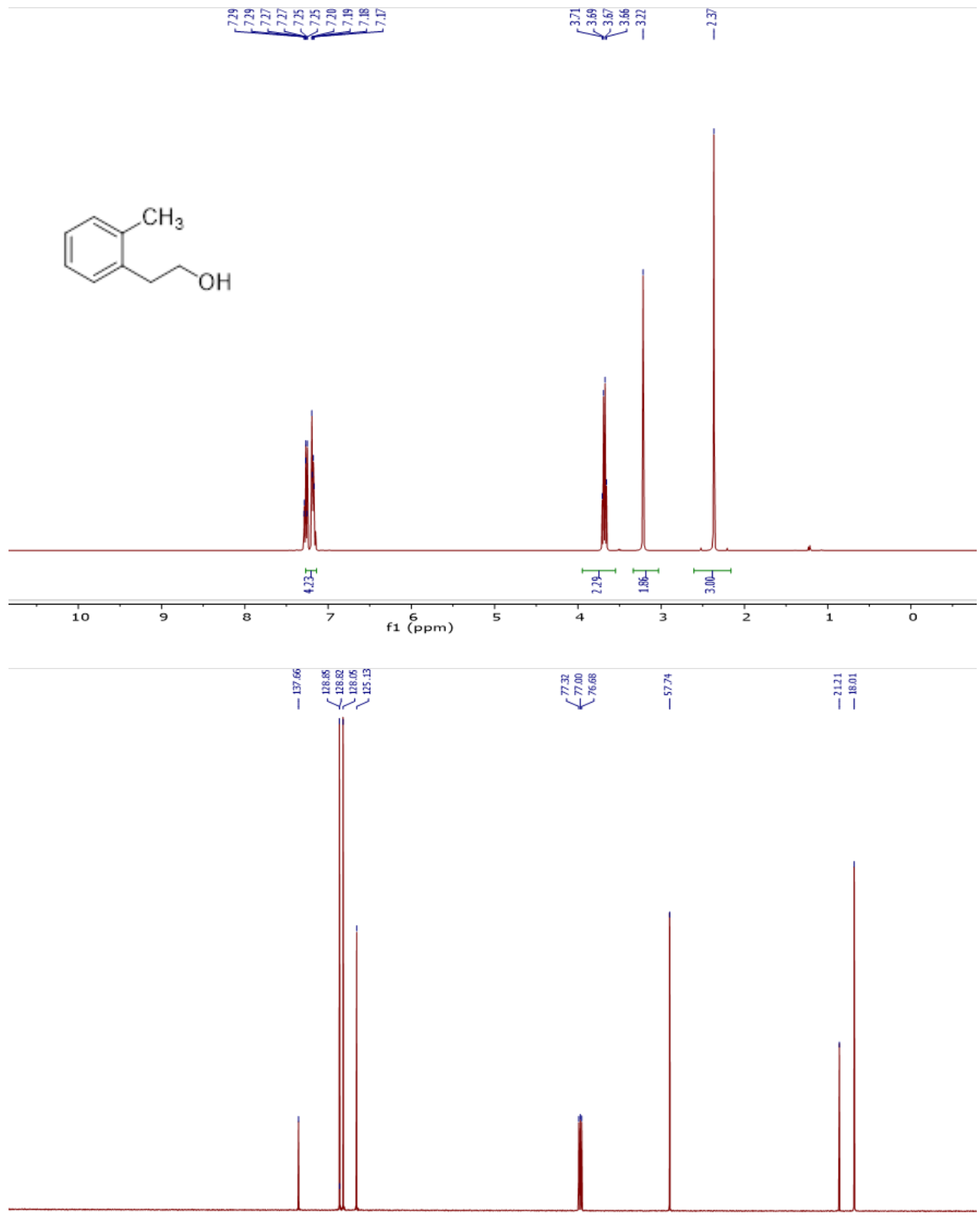

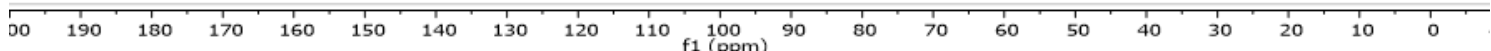


4-Fluoro-4'-methylbiphenyl $40\left(\mathrm{CDCl}_{3}, 400 \mathrm{MHz}\right.$ for $\left.{ }^{1} \mathrm{H} \mathrm{NMR}\right)$
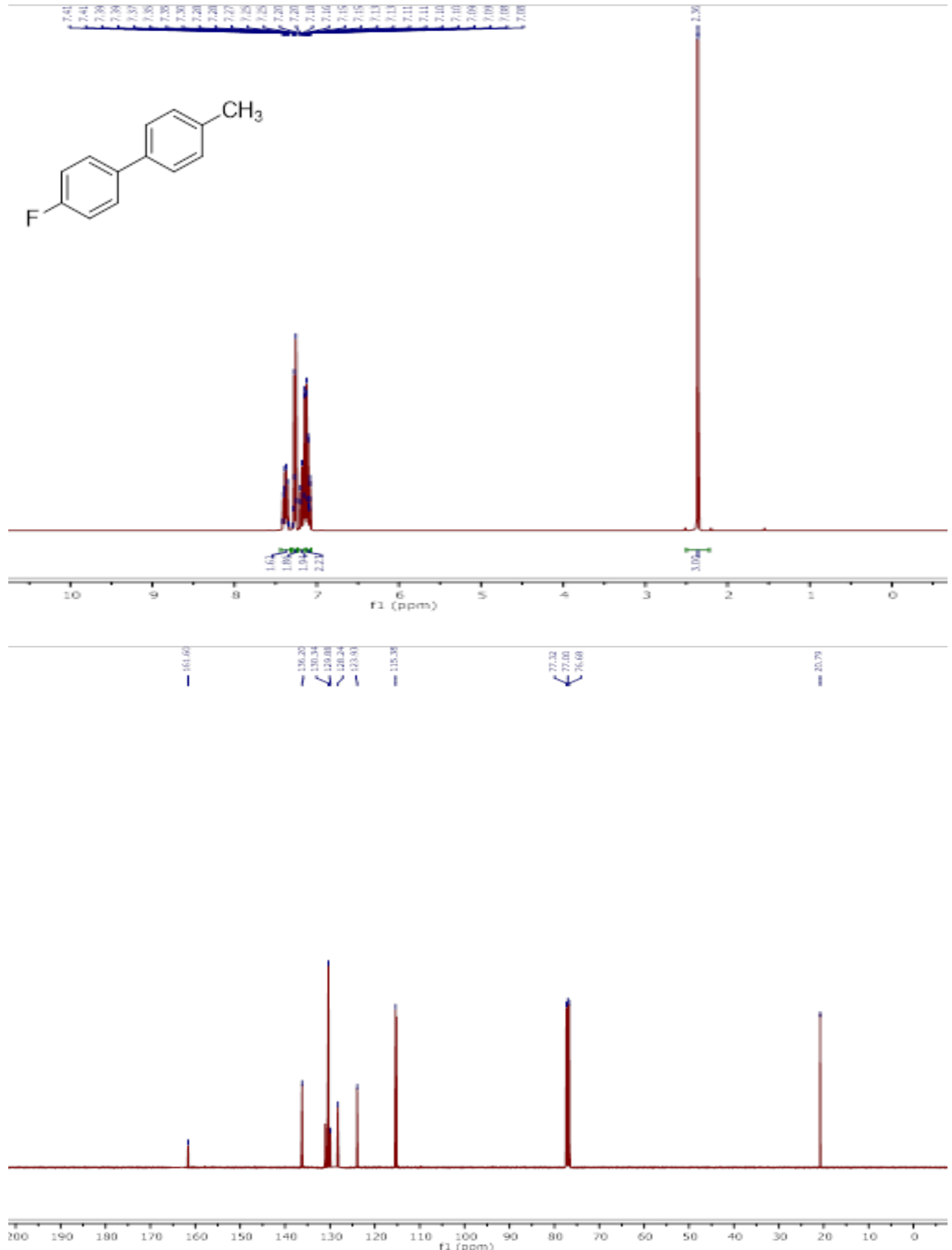
4-Methyl-4'-(trifluoromethyl)-1,1'-biphenyl 41 ( $\mathrm{CDCl}_{3}, 400 \mathrm{MHz}$ for ${ }^{1} \mathrm{H}$ NMR)
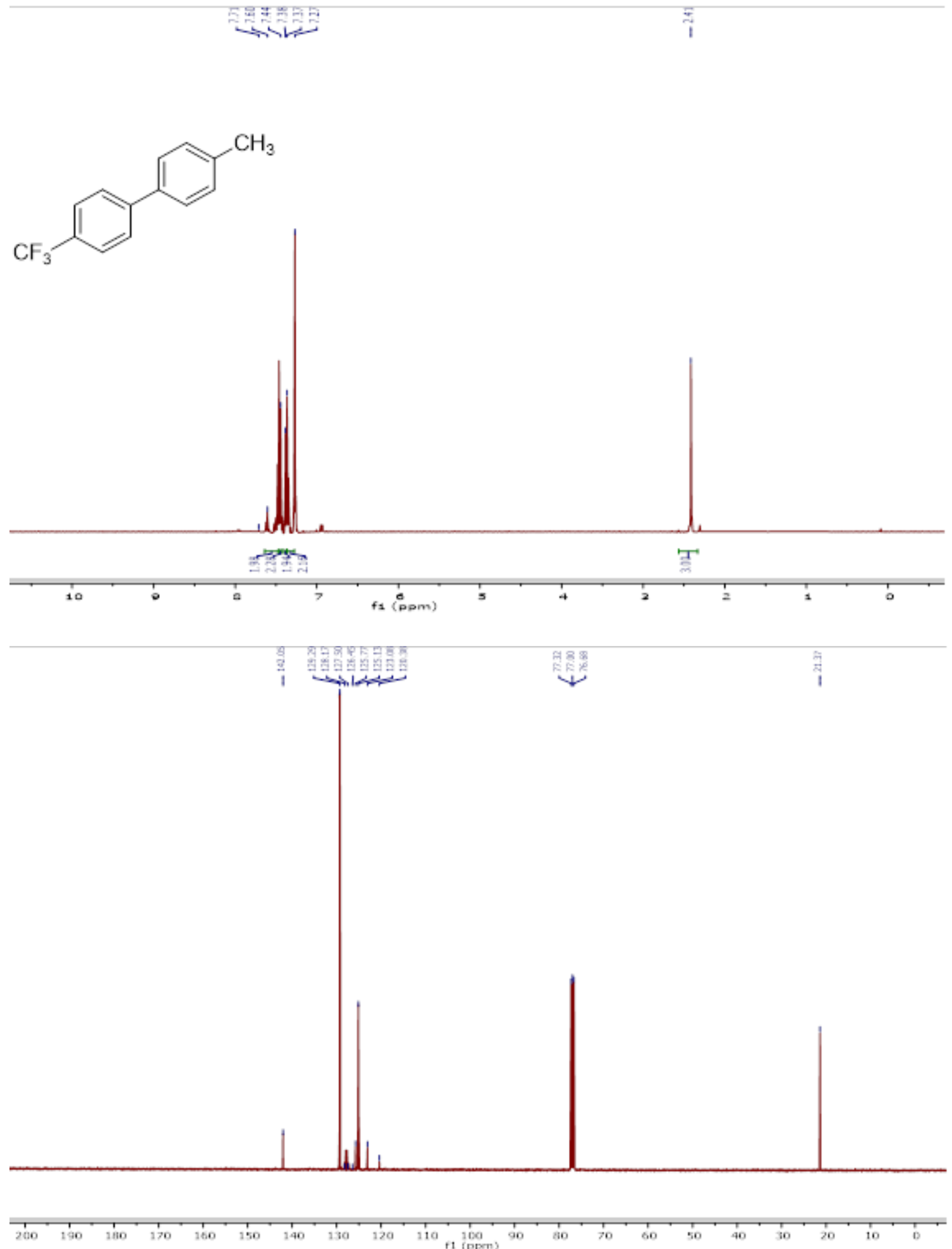
4-methyl-4'-nitrobiphenyl $42\left(\mathrm{CDCl}_{3}, 400 \mathrm{MHz}\right.$ for $\left.{ }^{1} \mathrm{H} \mathrm{NMR}\right)$
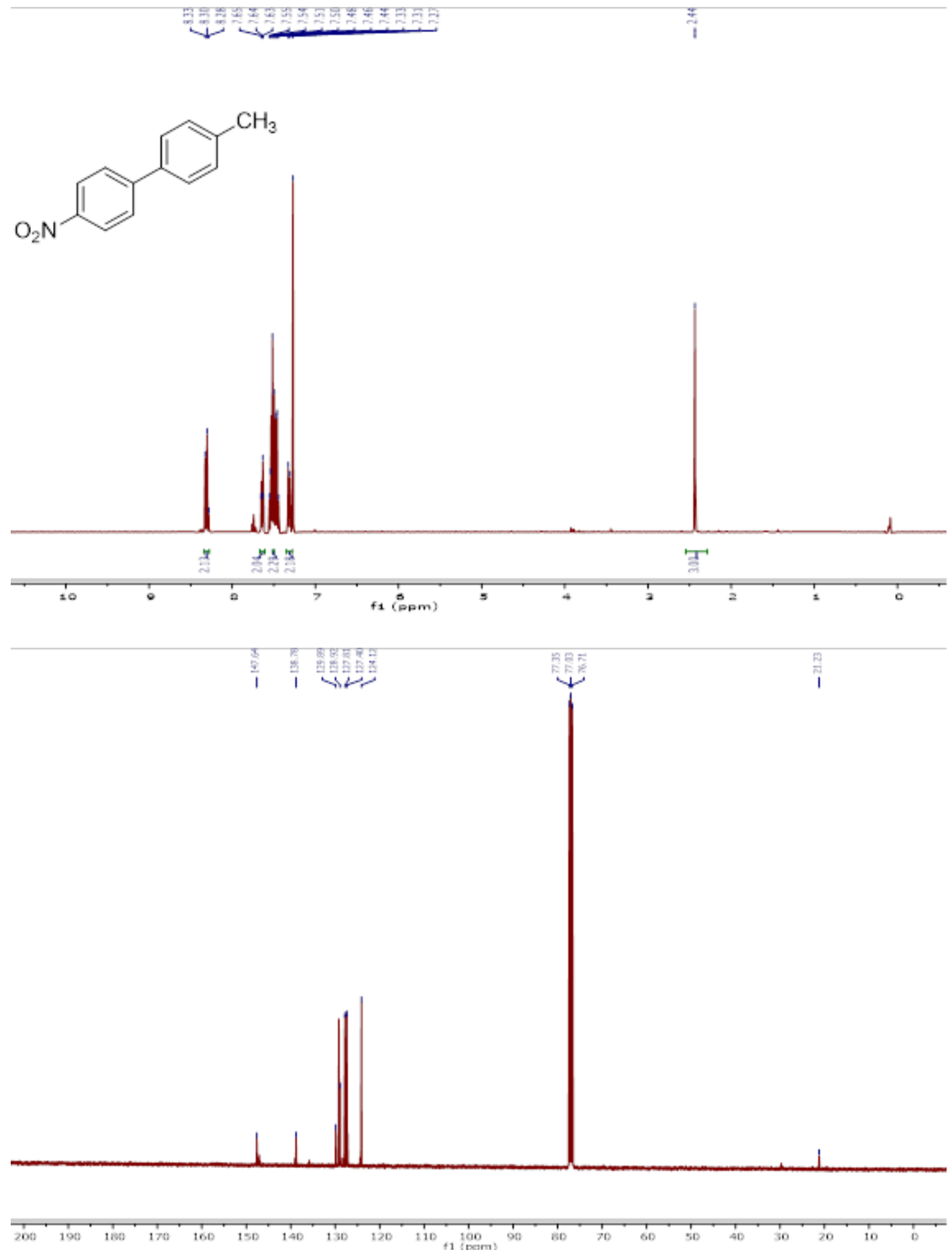
1-methyl-4-(3-pentyn-1-yloxy)-benzene $43\left(\mathrm{CDCl}_{3}, 400 \mathrm{MHz}\right.$ for $\left.{ }^{1} \mathrm{H} \mathrm{NMR}\right)$
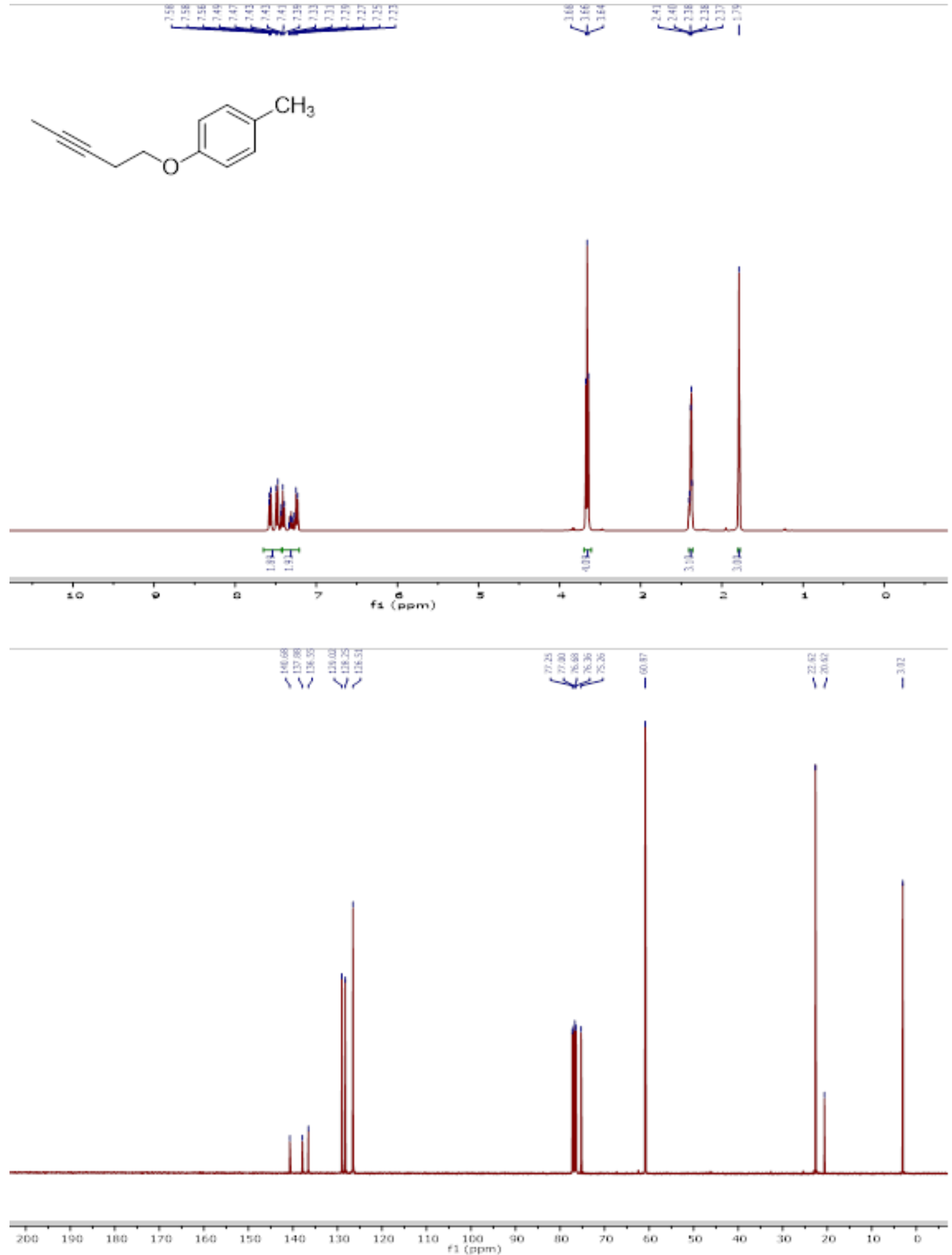
2-(4-Methylphenyl)furan $44\left(\mathrm{CDCl}_{3}, 400 \mathrm{MHz}\right.$ for ${ }^{1} \mathrm{H}$ NMR)
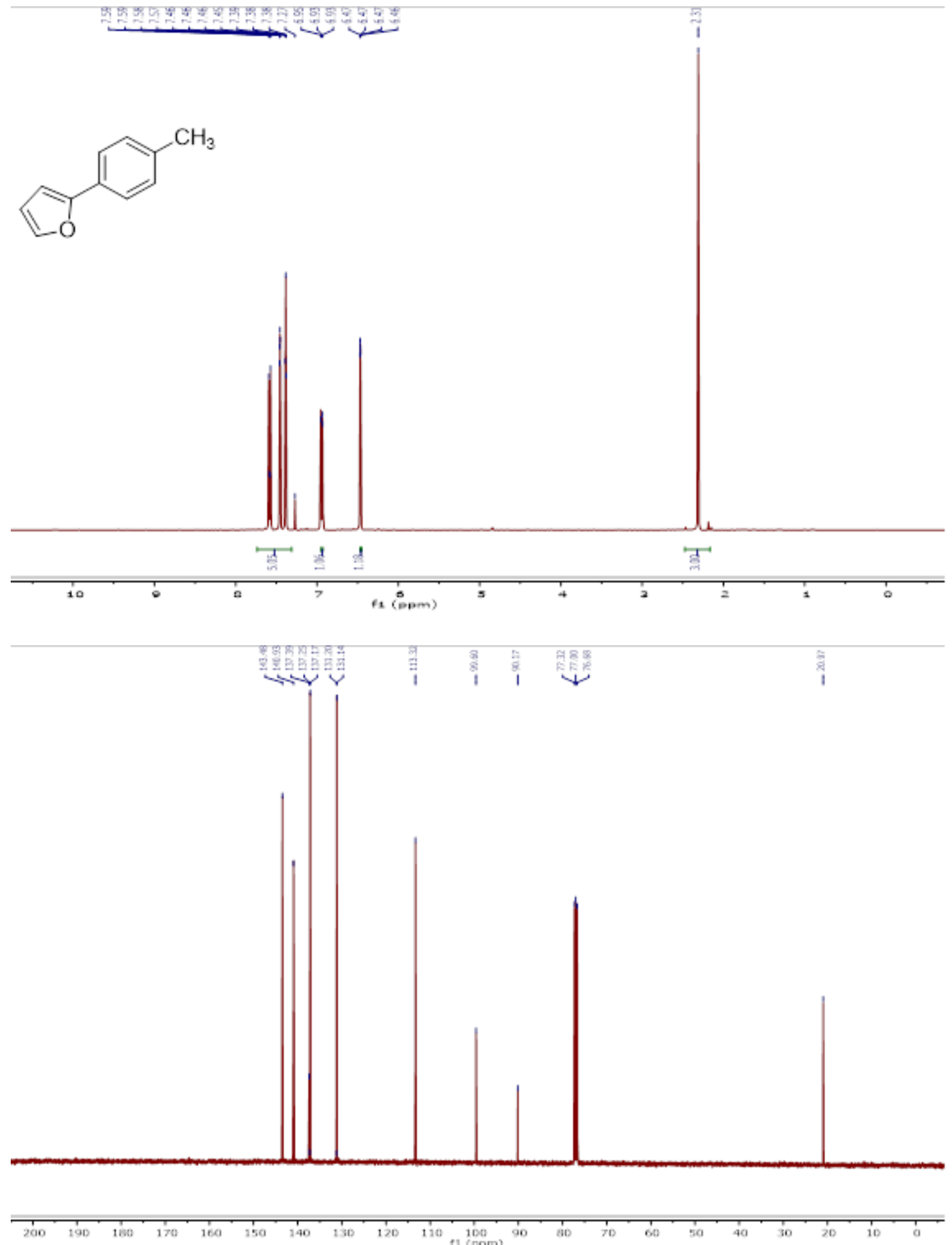
4-(1-propen-1-yl)-1,1'-biphenyl 45 ( $\mathrm{CDCl}_{3}, 400 \mathrm{MHz}$ for ${ }^{1} \mathrm{H}$ NMR)

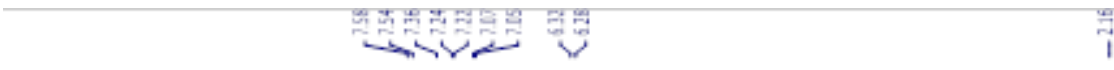<smiles>C/C=C/c1ccc(-c2ccccc2)cc1</smiles>

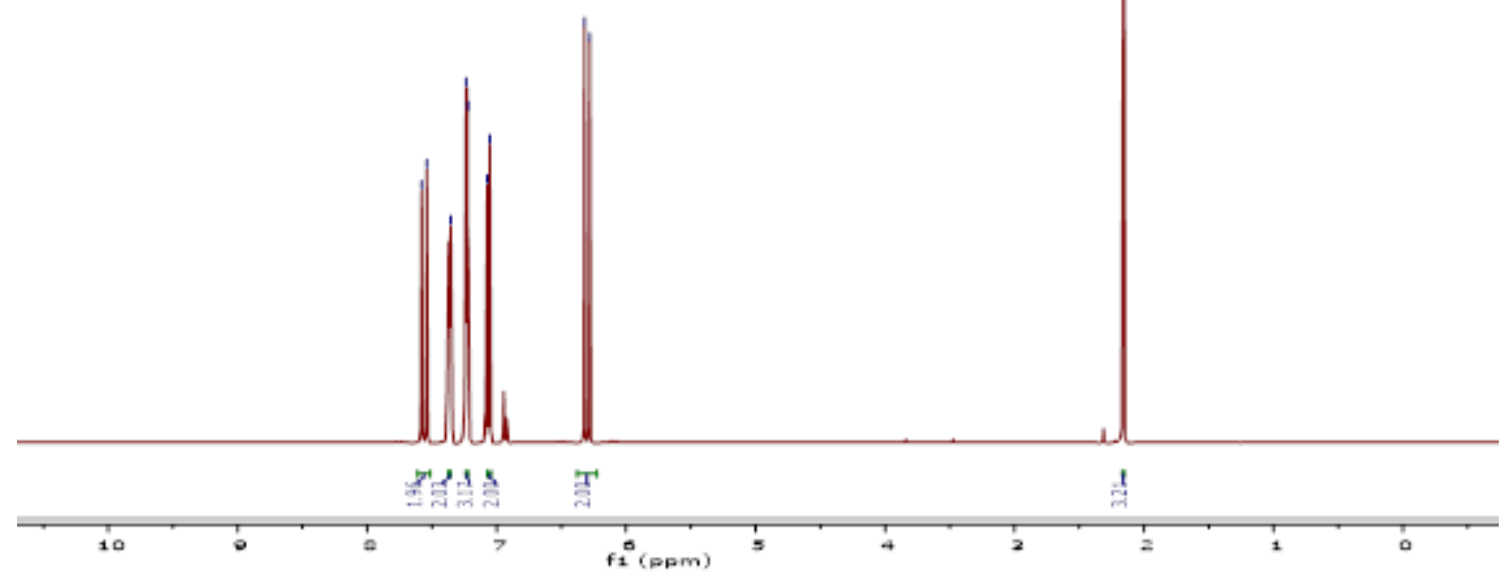

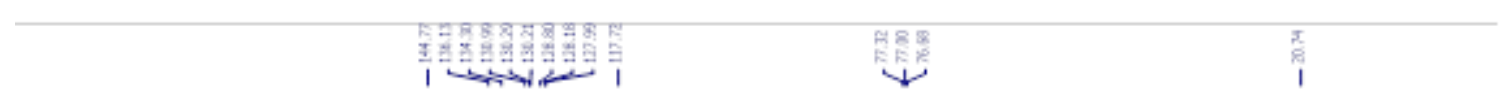
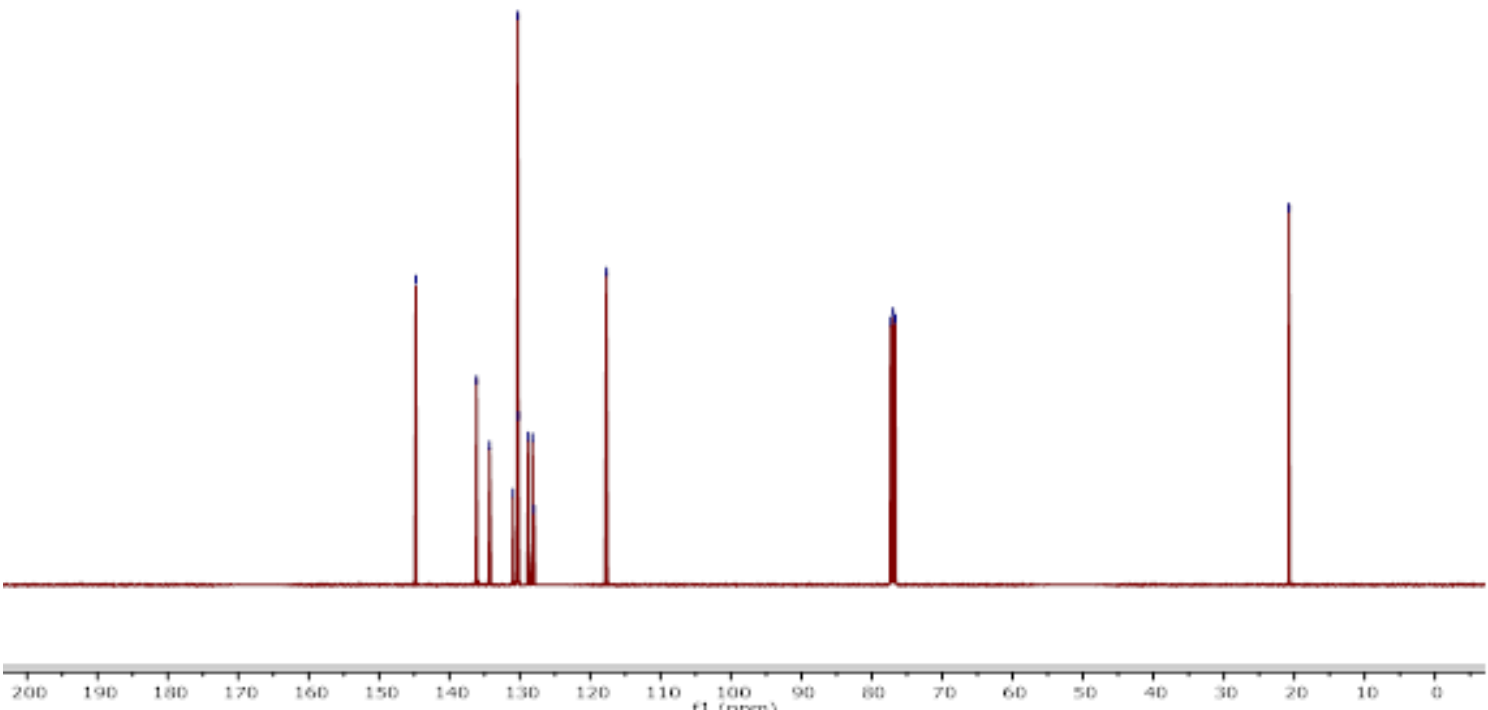
1,4-Dimethoxy-2-(methyl- $\left.d_{3}\right)$ benzene $46\left(\mathrm{CDCl}_{3}, 400 \mathrm{MHz}\right.$ for $\left.{ }^{1} \mathrm{H} \mathrm{NMR}\right)$

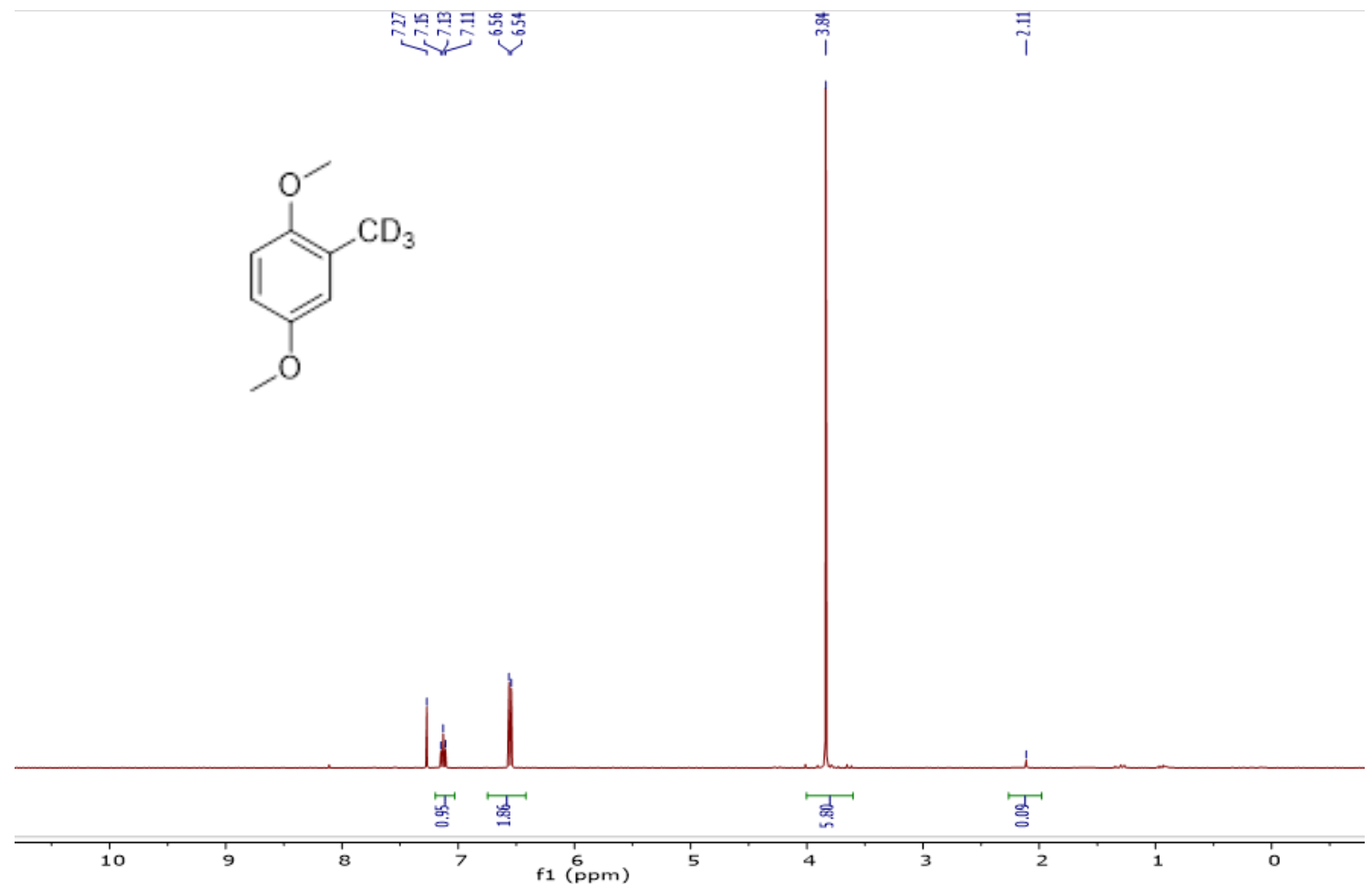

$d^{3}$-1-(trans-4-butylcyclohexyl)-4-methylbenzene $47\left(\mathrm{CDCl}_{3}, 400 \mathrm{MHz}\right.$ for $\left.{ }^{1} \mathrm{H} \mathrm{NMR}\right)$
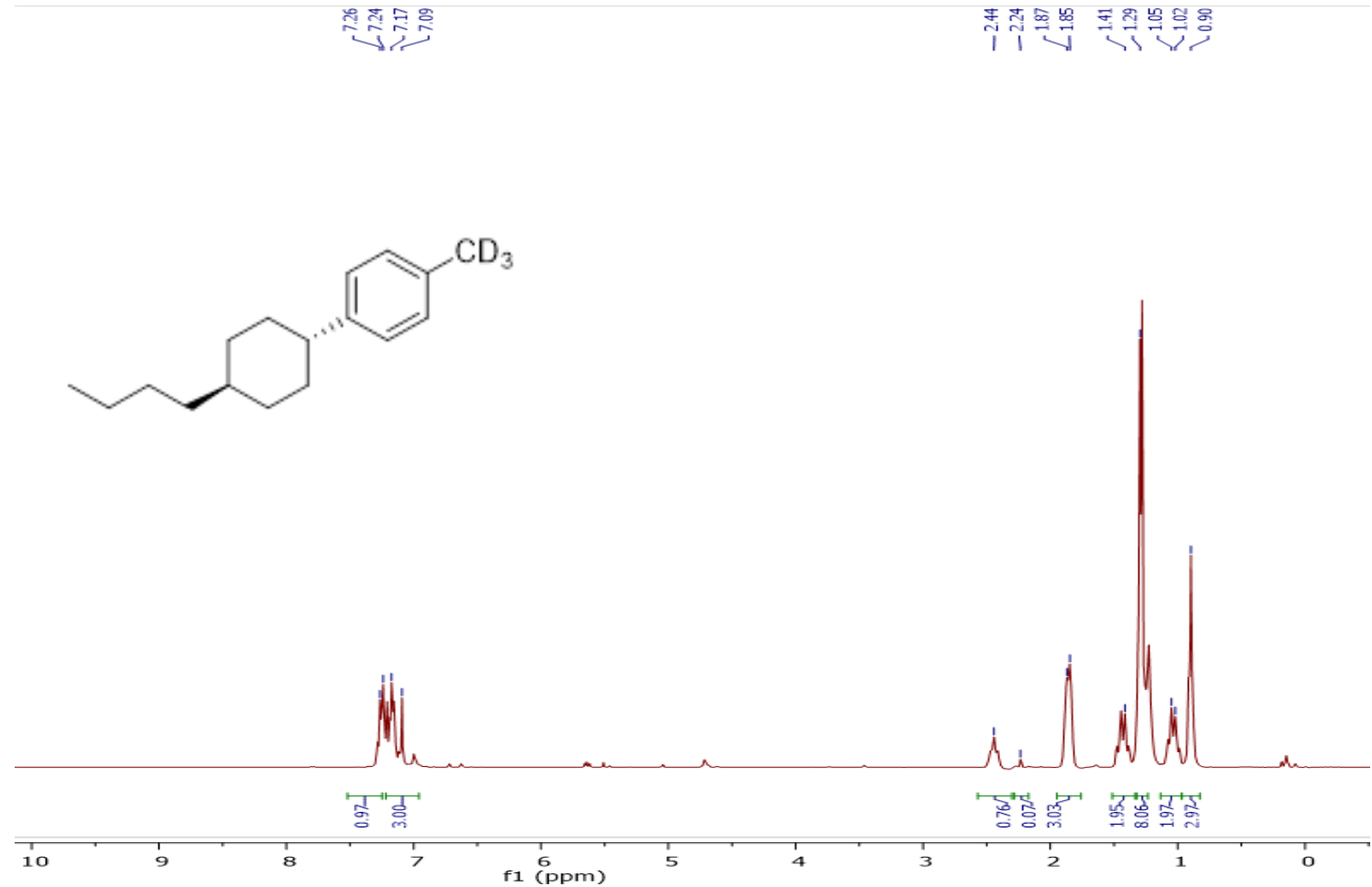
$d^{3}$-2-Methylnapthalene $48\left(\mathrm{CDCl}_{3}, 400 \mathrm{MHz}\right.$ for $\left.{ }^{1} \mathrm{H} \mathrm{NMR}\right)$<smiles>O=C(c1ccccc1)c1ccc2ccccc2c1</smiles>

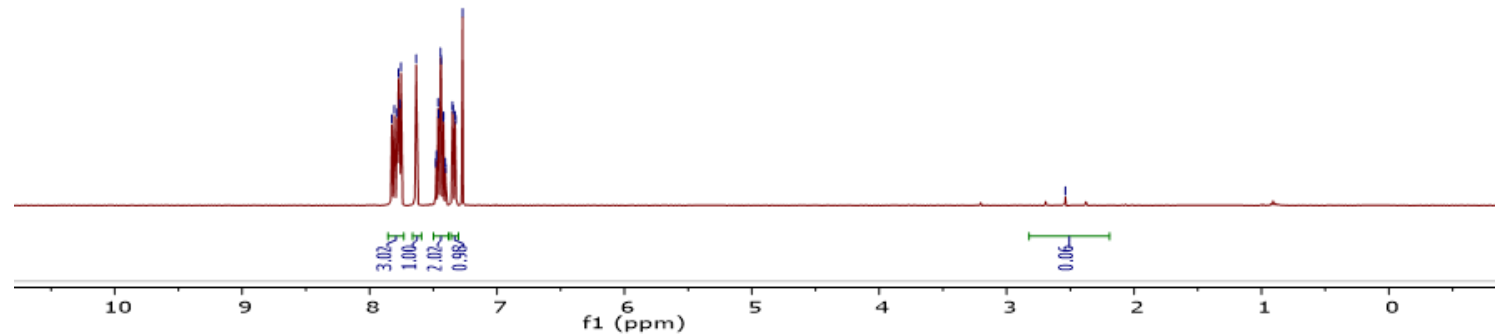

$d^{3}$-1,6-Dimethylindole $49\left(\mathrm{CDCl}_{3}, 400 \mathrm{MHz}\right.$ for ${ }^{1} \mathrm{H}$ NMR)

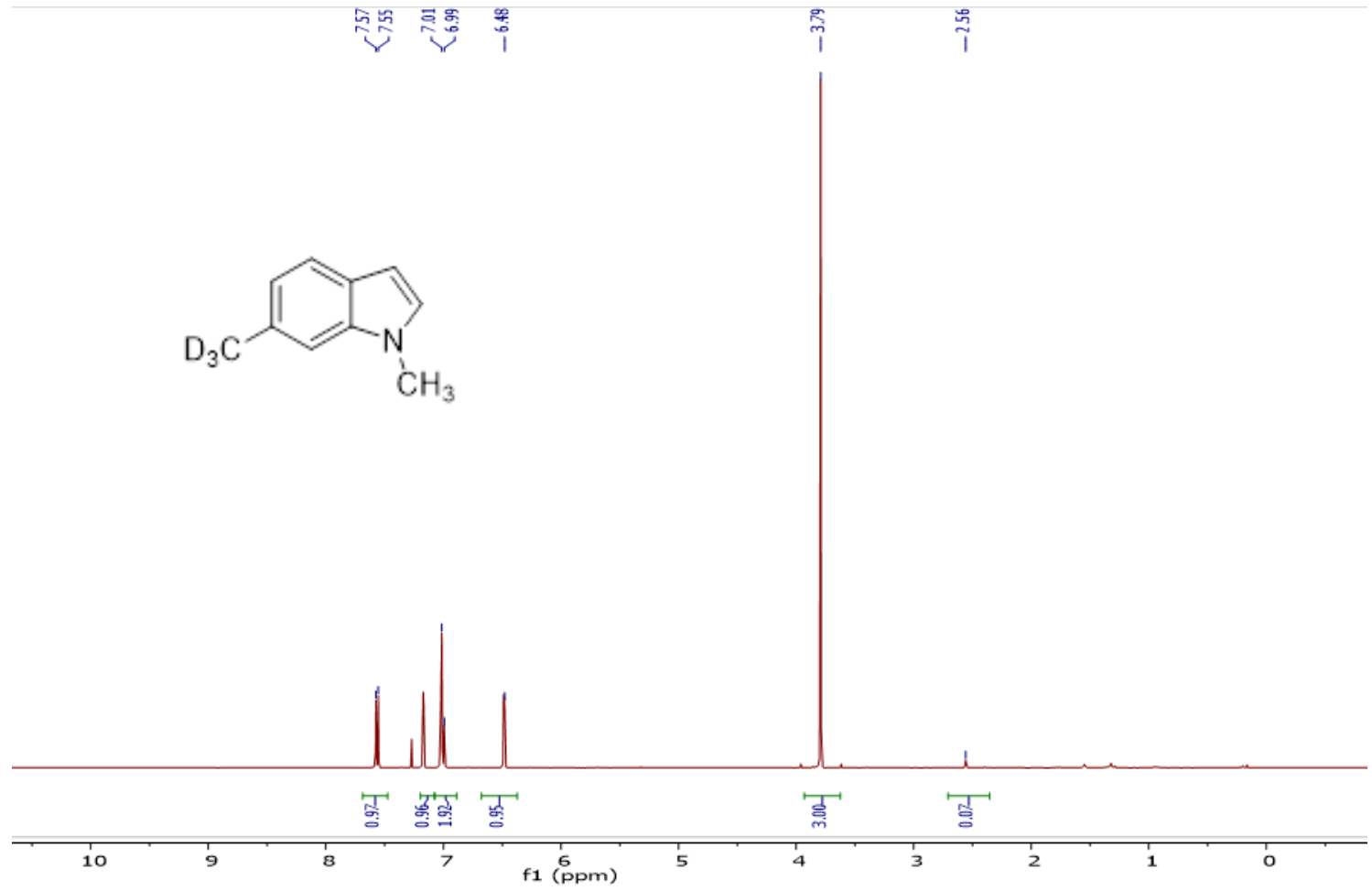


2-(2-Methoxyphenyl)-5-methylpyridine $54\left(\mathrm{CDCl}_{3}, 400 \mathrm{MHz}\right.$ for ${ }^{1} \mathrm{H} \mathrm{NMR}, 100 \mathrm{MHz}$ for $\left.{ }^{13} \mathrm{C} \mathrm{NMR}\right)$

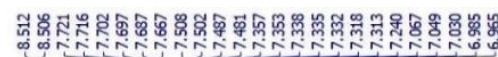

蔡
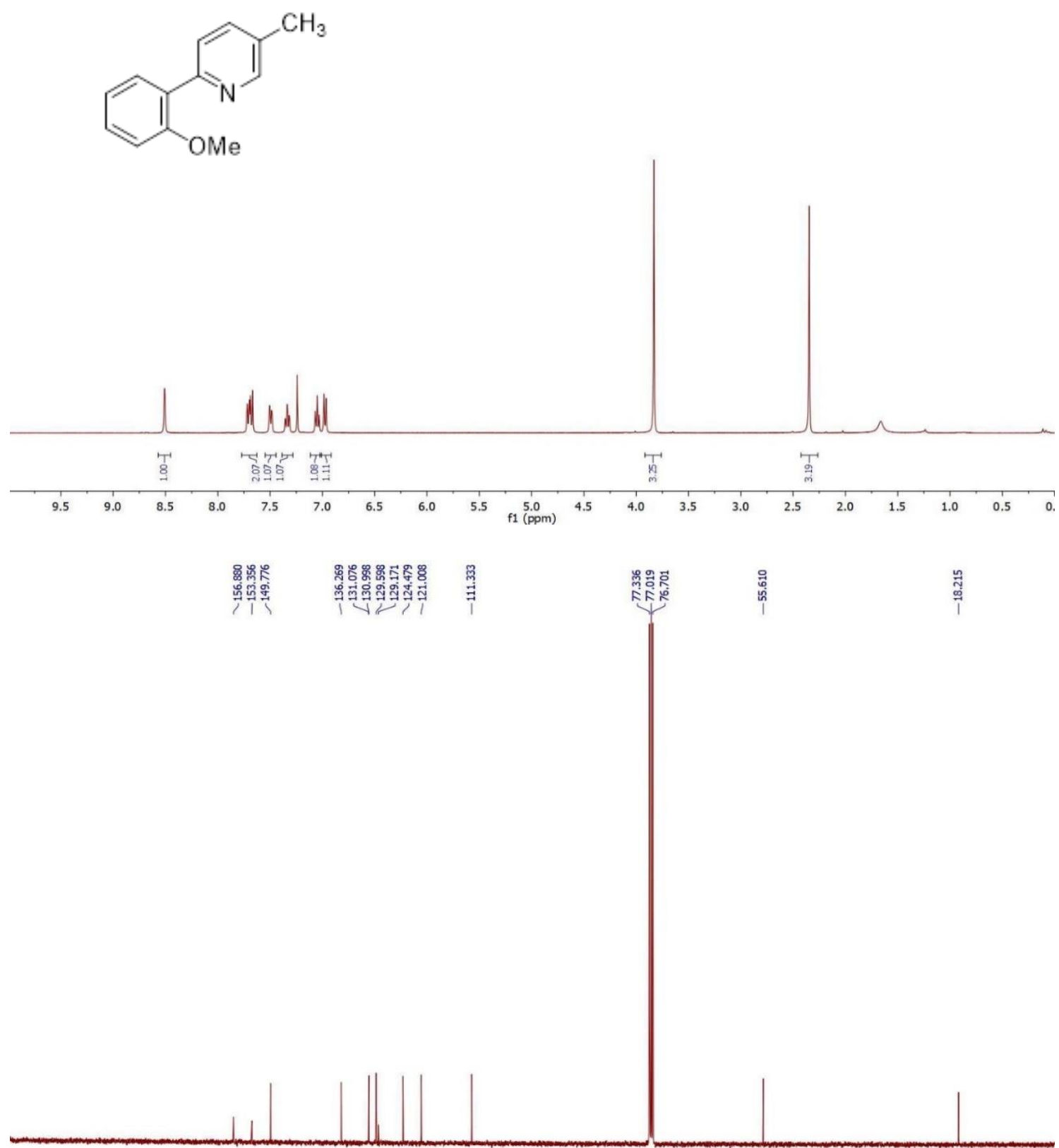

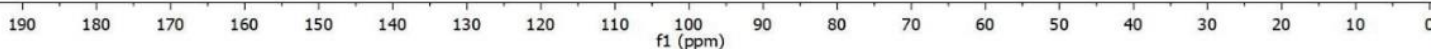


2-Methoxy-6-methylnaphthalene $55\left(\mathrm{CDCl}_{3}, 400 \mathrm{MHz}\right.$ for ${ }^{1} \mathrm{H} \mathrm{NMR}, 100 \mathrm{MHz}$ for $\left.{ }^{13} \mathrm{C} \mathrm{NMR}\right)$

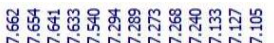

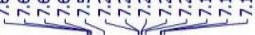

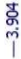

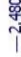
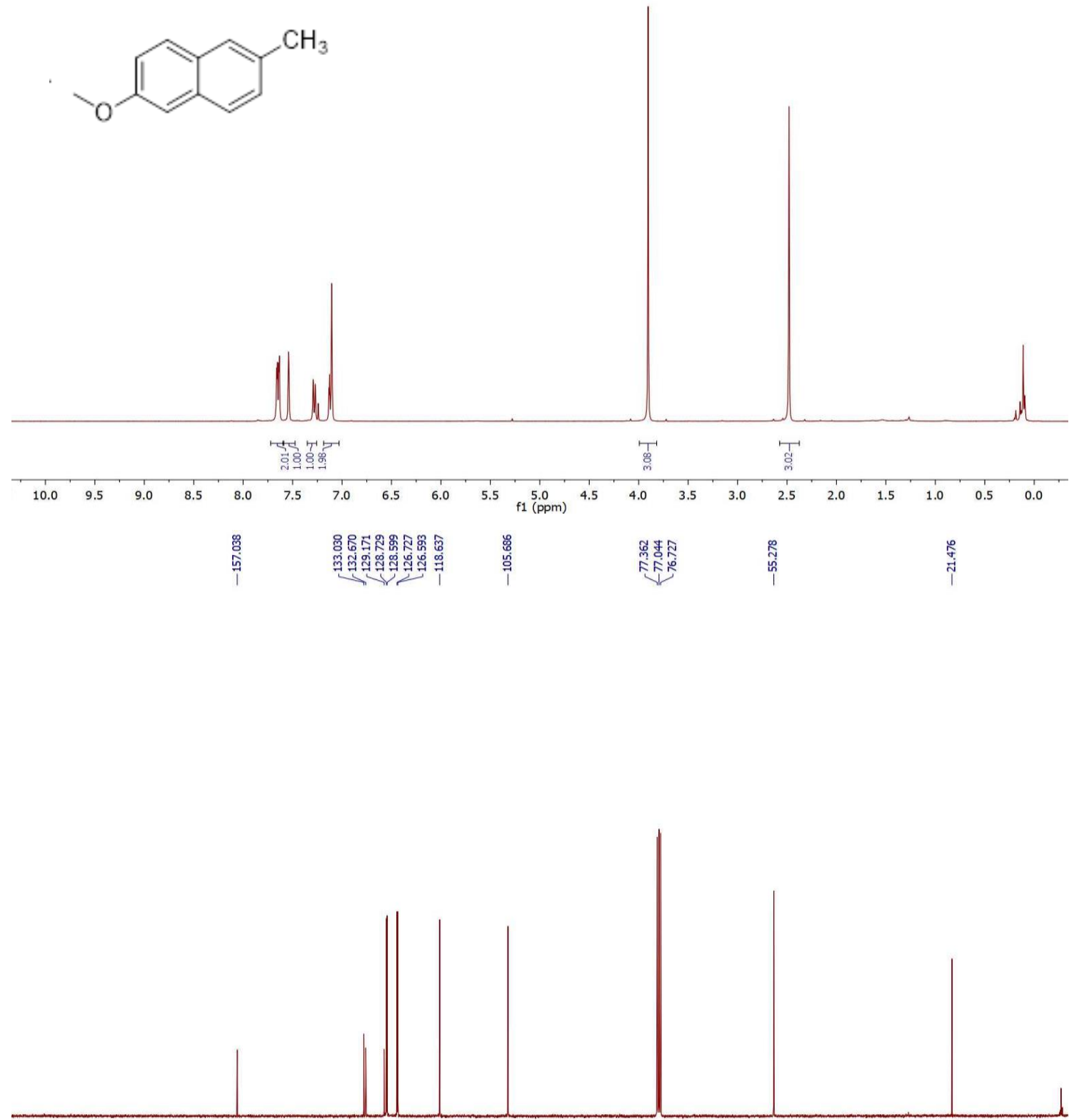

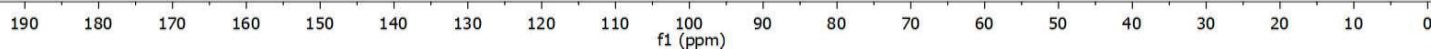


2-Methoxy-3-methylnapthalene $56\left(\mathrm{CDCl}_{3}, 400 \mathrm{MHz}\right.$ for ${ }^{1} \mathrm{H} \mathrm{NMR}, 100 \mathrm{MHz}$ for $\left.{ }^{13} \mathrm{C} \mathrm{NMR}\right)$
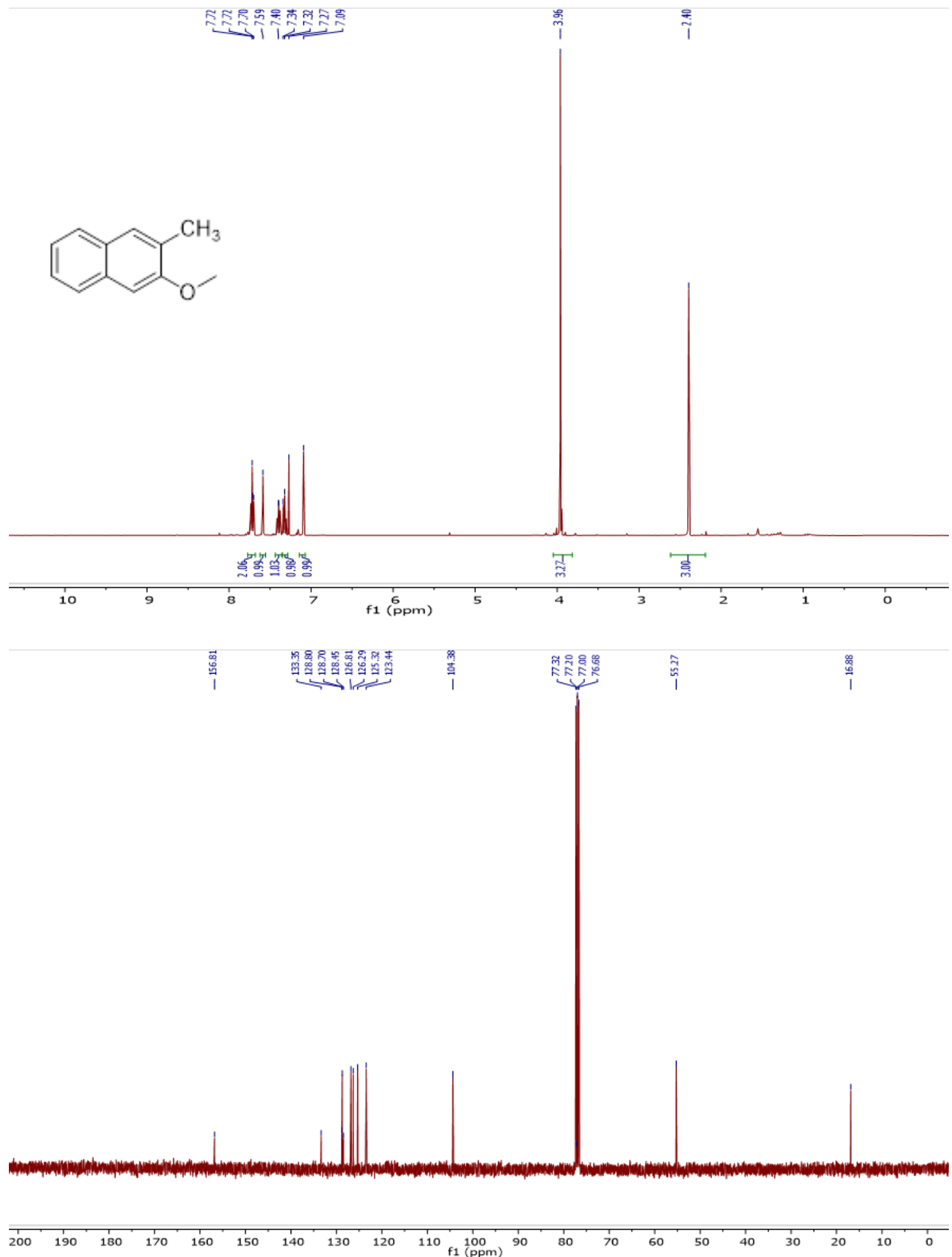
1,3-Dimethoxy-2-methylbenzene $57\left(\mathrm{CDCl}_{3}, 400 \mathrm{MHz}\right.$ for ${ }^{1} \mathrm{H} \mathrm{NMR}, 100 \mathrm{MHz}$ for $\left.{ }^{13} \mathrm{C} \mathrm{NMR}\right)$

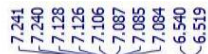
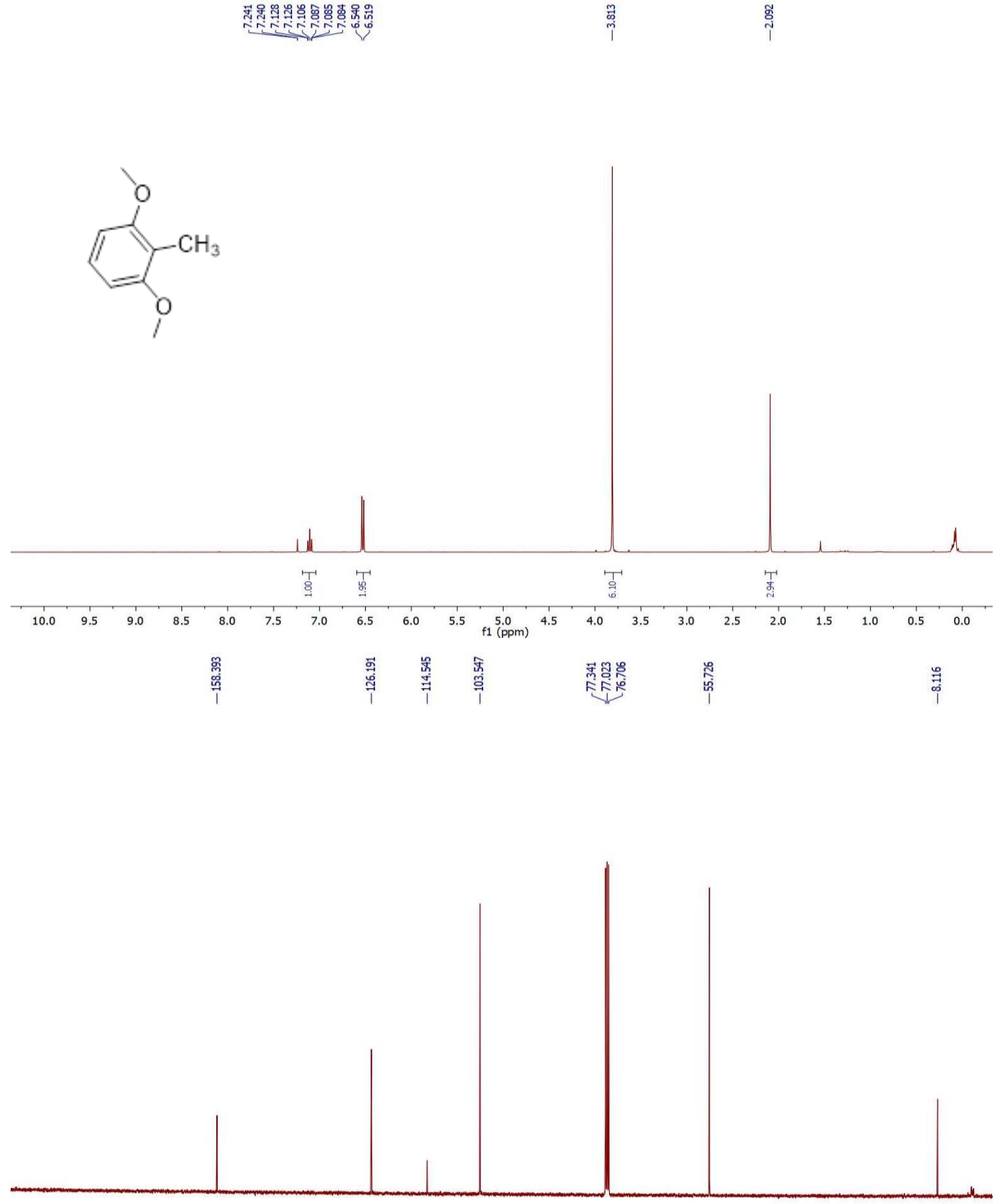
$110 \mathrm{f} 1 \stackrel{100}{(\mathrm{ppm})}$

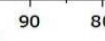


Methyl 5-phenylpyridine-3-carboxylate $58\left(\mathrm{CDCl}_{3}, 400 \mathrm{MHz}\right.$ for ${ }^{1} \mathrm{H} \mathrm{NMR}, 100 \mathrm{MHz}$ for $\left.{ }^{13} \mathrm{C} \mathrm{NMR}\right)$

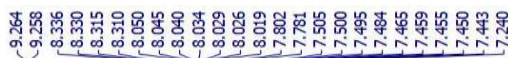<smiles>CC(=O)c1ccc(-c2ccccc2)nc1</smiles>
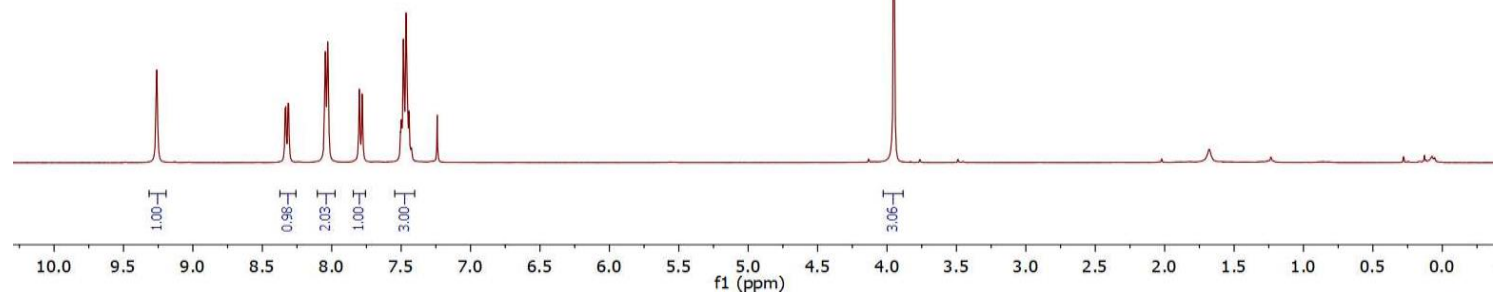

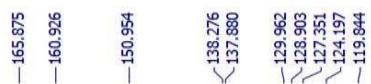
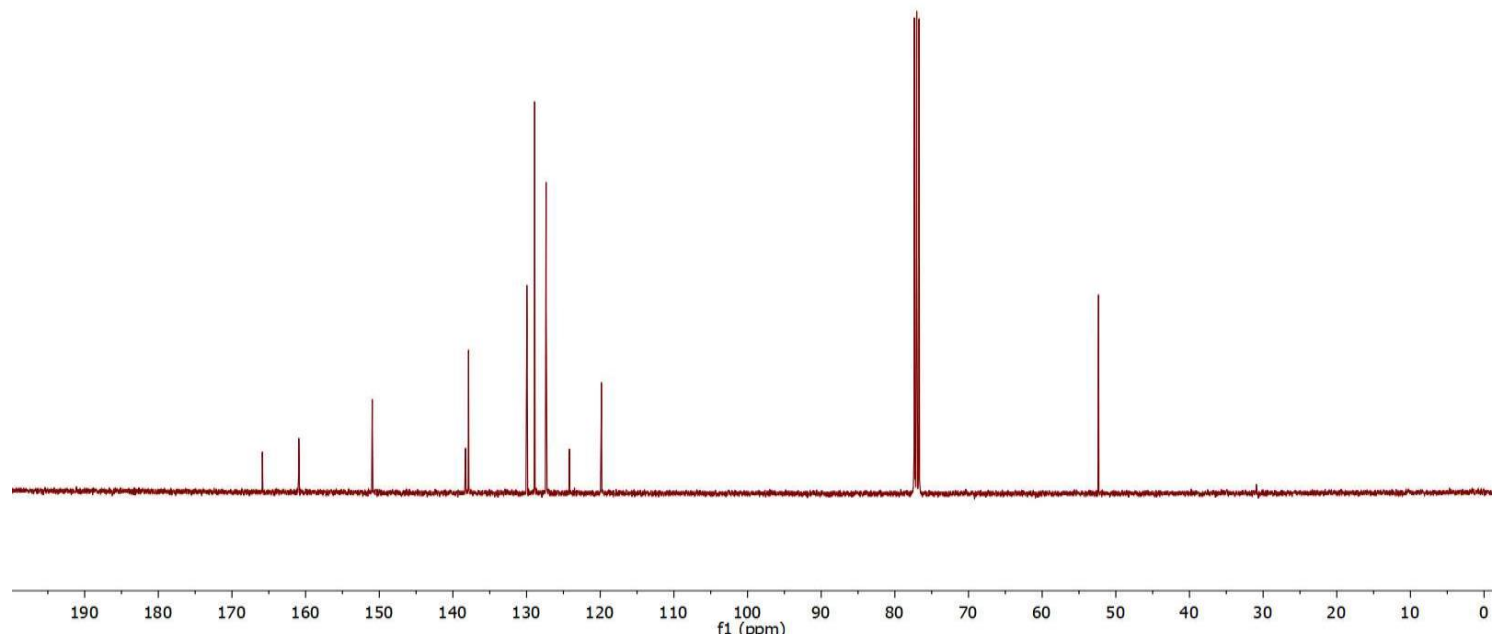
2-Methyl naphthoate $59\left(\mathrm{CDCl}_{3}, 400 \mathrm{MHz}\right.$ for ${ }^{1} \mathrm{H}$ NMR, $100 \mathrm{MHz}$ for $\left.{ }^{13} \mathrm{C} \mathrm{NMR}\right)$

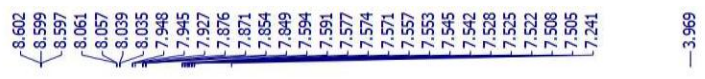
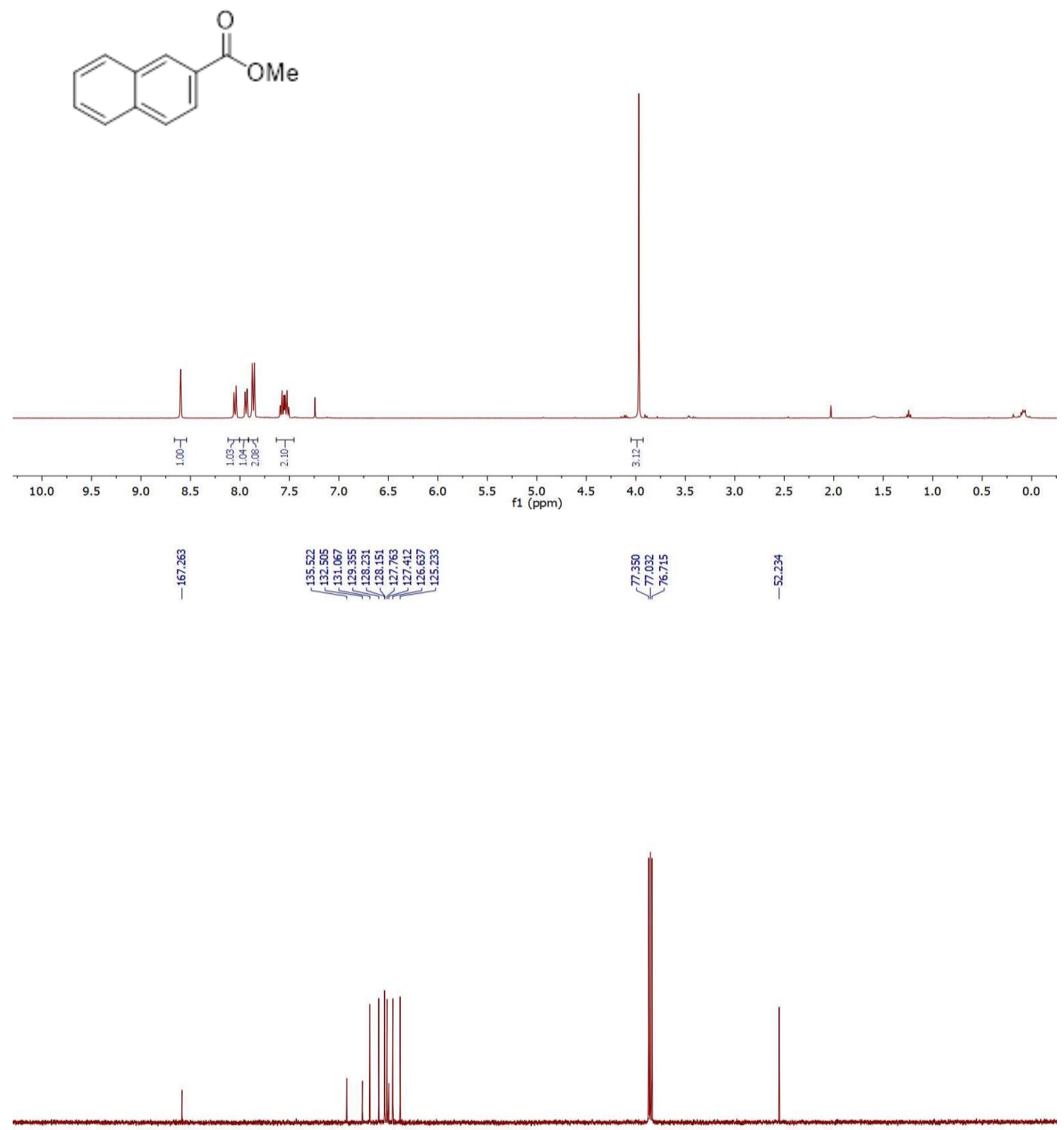

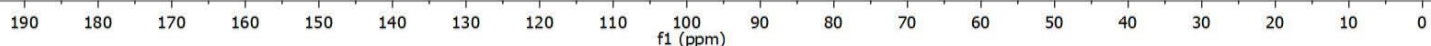


Methyl-2-methoxybenzoate $60\left(\mathrm{CDCl}_{3}, 400 \mathrm{MHz}\right.$ for ${ }^{1} \mathrm{H} \mathrm{NMR}, 100 \mathrm{MHz}$ for $\left.{ }^{13} \mathrm{C} \mathrm{NMR}\right)$
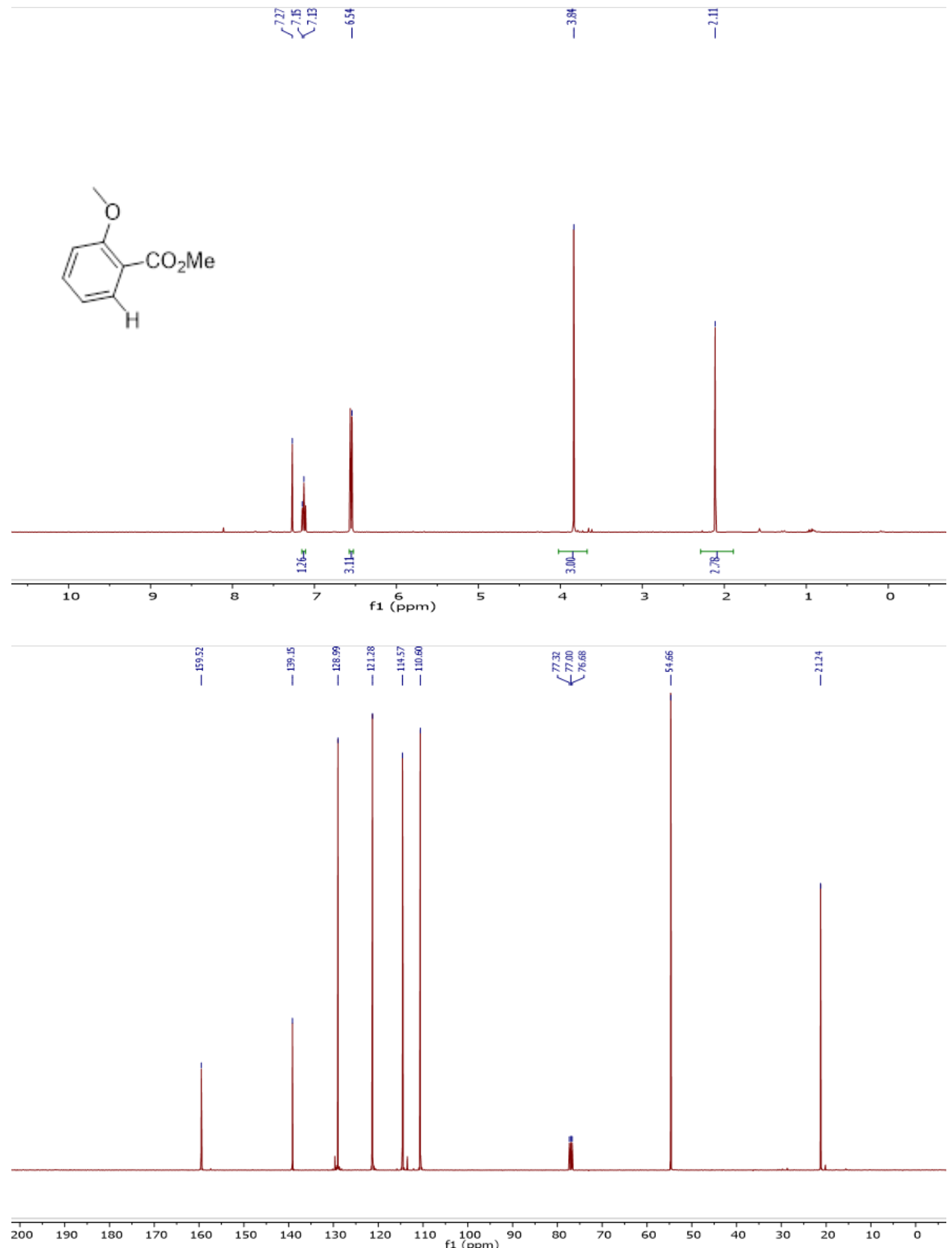
4-[[(1,1-dimethylethyl)dimethylsilyl]oxy]-4'-methyl-1,1'-biphenyl S20 ( $\mathrm{CDCl}_{3}, 400 \mathrm{MHz}$ for ${ }^{1} \mathrm{H}$ NMR, 100 $\mathrm{MHz}$ for $\left.{ }^{13} \mathrm{CNMR}\right)$

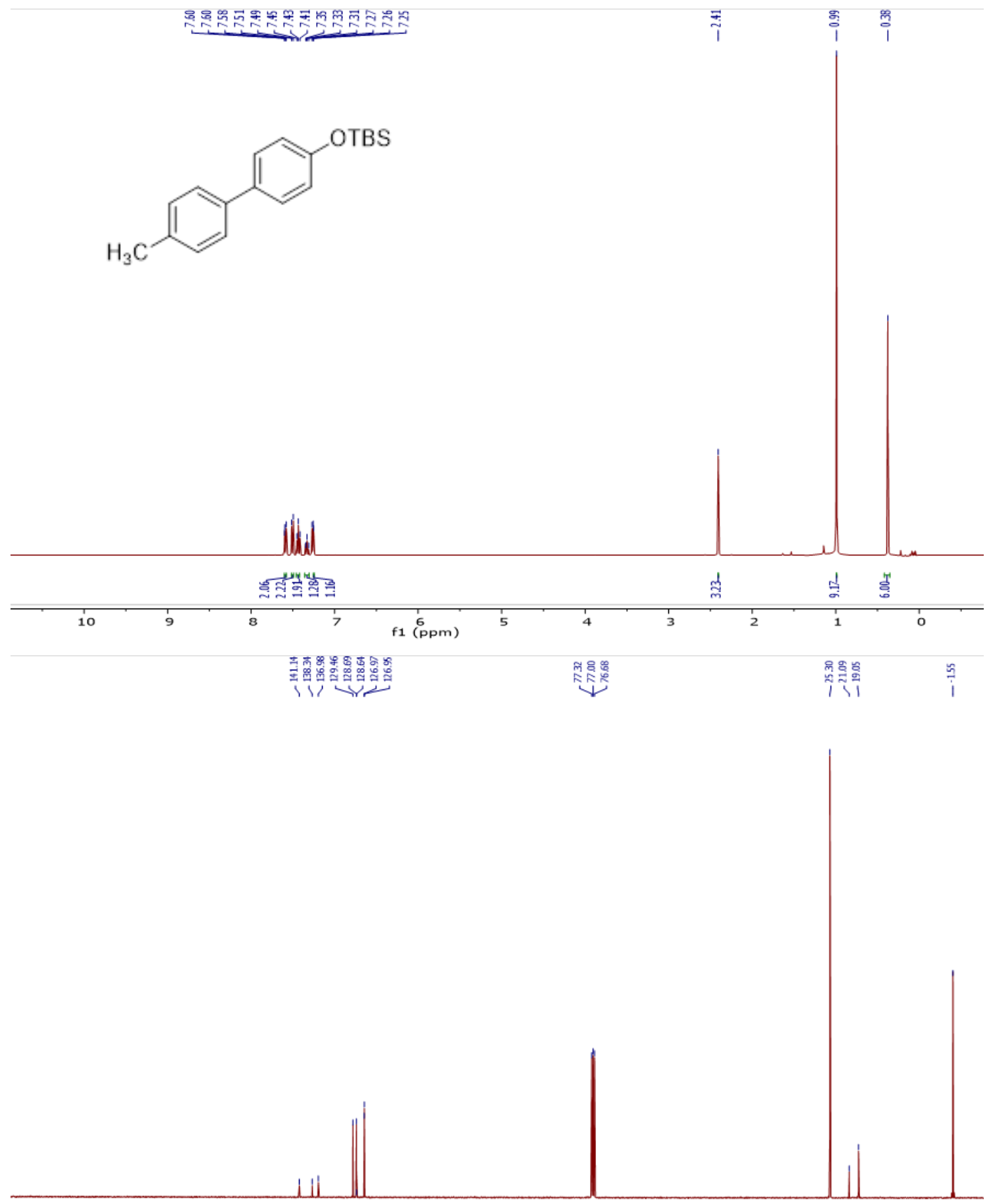

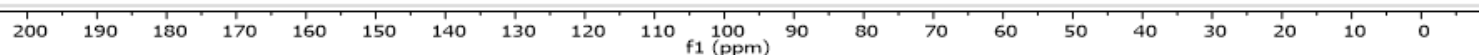


4,4'-Oxybis(methylbenzene) $56\left(\mathrm{CDCl}_{3}, 400 \mathrm{MHz}\right.$ for ${ }^{1} \mathrm{H} \mathrm{NMR}, 100 \mathrm{MHz}$ for ${ }^{13} \mathrm{C} \mathrm{NMR}$ )

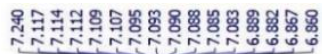<smiles>Cc1ccc(Oc2ccc(C)cc2)cc1</smiles>
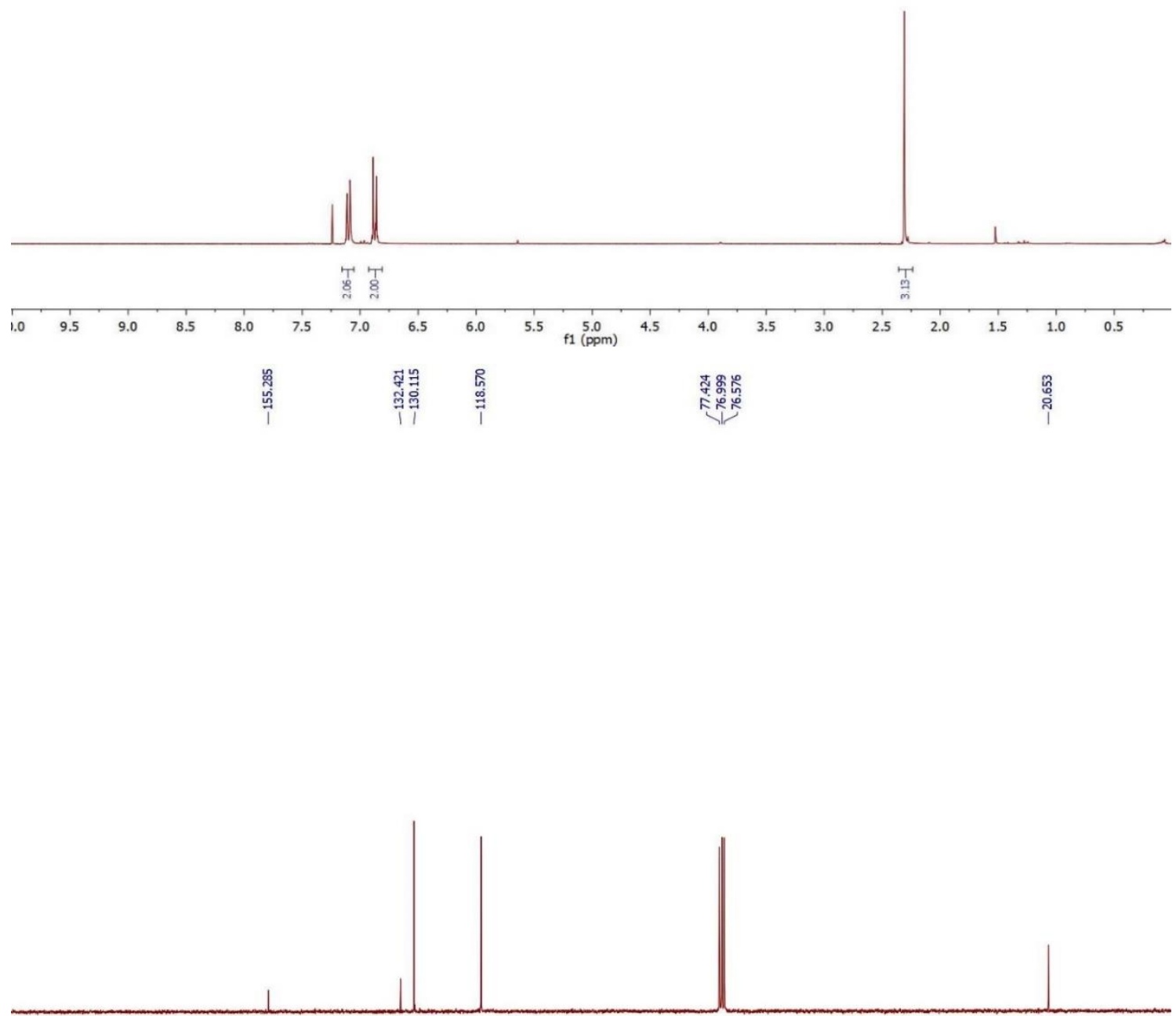


\section{References}

1) Hans, M.; Lorkowski, J.; Demonceau, A.; Delaude, L. Beilstein. J. Org. Chem. 2015, 11, 2318-2325.

2) Nishizawa, R.; Nishiyama, T.; Hisaichi, K.; Hirai, K.; Habashita, H.; Takaoka, Y.; Tada, H.; Sagawa, K.; Shibayama, S.; Maeda, K.; Mitsuya, H.; Nakai, H.; Fukushima, D.; Toda, M. Bioorg. Med. Chem. 2010, 18, 5208-5223.

3) Nimmagadda, S. K.; Liu, M.; Karunananda, M. K.; Gao, D.-W.; Apolinar, O.; Chen, J. S.; Liu, P.; Engle, K. M. Angew. Chem. Int. Ed. 2019, 58, 3923-3927.

4) In, I.-K.; Lee, M. S.; Yang, J. E.; Kwak, J. H.; Lee, H.; Boovanahalli, S. K.; Lee, K.; Kim, S. J.; Moon, S. K.; Lee, S.; Choi, N. S.; Ahn, S. K.; Jung, J. K. Bioorg. Med. Chem. Lett. 2007, 17, 1799-1802.

5) Wei, Y.; Yoshikai, N. Org. Lett. 2011, 13, 5504-5507.

6) Yu, M.; Lizarzaburu, M.; Beckmann, H.; Connors, R.; Dai, K.; Haller, K.; Li, C.; Liang, L.; Lindstrom, M.; Ma, J.; Motani, A.; Wanska, M.; Zhang, A.; Li, L.; Medina, J. C. Bioorg. Med. Chem. Lett. 2010, $20,1758-1762$.

7) Nuss, J. M.; Harrison, S. D.; Ring, D. B.; Boyce, R. S.; Johnson, K.; Pfister, K. B.; Ramurthy, S.; Seely, L.; Wagman, A. S.; Desai, M.; Levine, B. H.; US 2002/0156087 A1, Oct. 24, 2002.

8) Delorme, D.; Zhou, Z. US 2004/0142953 A1, Jul. 22, 2004.

9) Alvarez-Bercedo, P.; Martin, R. J. Am. Chem. Soc. 2010, 132, 17352-17353.

10) Wen, L.; Tang, L.; Yang, Y.; Zha, Z.; Wang, Z. Org. Lett. 2016, 18, 1278-1281.

11) Schuster, C.; Börger, C.; Julich-Gruner, K. K.; Hesse, R.; Jäger, A.; Kaufmann, G.; Schmidt, A. W.; Knölker, H. Eur. J. Org. Chem., 2014, 4741-4752.

12) Liégault, B.; Lee, D.; Huestis, M. P.; Stuart, D. R.; Fagnou, K. J. Org. Chem. 2008, 73, 5022-5028.

13) Yamada, M.; Shio, Y.; Akiyama, T.; Honma, T.; Ohki, Y.; Takahashi, N.; Murai, K.; Arisawa, M. Green. Chem. 2019, 21, 4541-4549.

14) Hokamp, T.; Dewanji, A.; Lübbesmeyer, M.; Mück-Lichtenfeld, C.; Würthwein, E.-U.; Studer, A. Angew. Chem. Int. Ed. 2017, 56, 13275-13278.

15) Catti, L.; Tiefenbacher, K. Angew. Chem. Int. Ed. 2018, 57, 14589-14592.

16) Liu, X.-F.; Li, X.-Y.; Qiao, C.; Fu, H.-C.; He, L.-N. Angew. Chem. Int. Ed. 2017, 56, 7425-7429.

17) Yao, C. Z.; Li, Q. Q.; Wang, M. M.; Ning, X. S.; Kang, Y. B. Chem. Commun. 2015, 51, 7729-7732.

18) Cella, R.; Stefani, H. A. Tetrahedron 2006, 62, 5656-5662.

19) Wang, H.; Li, L.; Bai, X.-F.; Shang, J.-Y.; Yang, K.-F.; Xu, L.-W.; Adv. Synth. Catal. 2013, 355, 341347.

20) Zhao, Y.; Wang, X.; Kodama, K.; Hirose, T. ChemistrySelect, 2018, 3, 12620-12624. 
21) Huang, C.; Chernyak, N.; Dudnik, A. S.; Gevorgyan, V. Adv. Synth. Catal. 2011, 353, 1285-1305.

22) Frost, J. R.; Cheong, C. B.; Donohoe, T. J. Synthesis 2017, 49, 910-916.

23) Haydl, A. M.; Hartwig, J. F. Org. Lett. 2019, 21, 1337-1341.

24) Zhang, E.; Tang, J.; Li, S.; Wu, P.; Moses, J. E.; Sharpless, K. B. Chem. Eur. J. 2016, 22, 5692-5697.

25) Abid, O. R.; Nawaz, M.; Ibad, M. F.; Khera, R. A.; laroshenko, V.; Langer, P. Org. Biomol. Chem. 2011, 9, 2185-2191.

26) Lerma, I. S.; Cawley, M. J.; Cloke, F. G. N.; Arentsen, K.; Scott, J. S.; Pearson, S. E.; Hayler, J.; Caddick, S. J. Organomet. Chem. 2005, 690, 5841-5848.

27) Pirkl, N.; Grosso, A. D.; Mallick, B.; Doppiuc, A.; Gooßen, L. J. Chem. Commun. 2019, 55, 52755278.

28) Zhang, J.; Park, S.; Chang, S. J. Am. Chem. Soc. 2018, 140, 13209-13213.

29) Manolikakes, G.; Gavryushin, A. Knochel, P. J. Org. Chem. 2008, 73, 1429-1434.

30) Benjamin, S.; Brown, L. A.; Sames, D. J. Am. Chem. Soc. 2005, 127, 8050-8057.

31) Klare, H.; Oestreich, M.; Ito, J.; Nishiyama, H.; Ohki, Y.; Tatsumi, K. J. Am. Chem. Soc. 2011, 133, 3312-3315.

32) Patil, P. H.; Nallasivam, J. L.; Fernandes, R. A. Asian. J. Org. Chem. 2015, 4, 552-559.

33) Jordan-Hore, J. A.; Johansson, C. C. C.; Gulias, M.; Beck, E. M.; Gaunt, M. J. J. Am. Chem. Soc. 2008, $130,16184-16186$.

34) Hu, Z.-Y.; Zhang, Y.; Li, X.-C.; Zi, J.; Guo, X.-X; Org. Lett. 2019, 21, 989-992.

35) Counceller, C. M.; Eichman, C. C.; Wray, B. C.; Stambuli, J. P. Org. Lett. 2008, 105, 1021-1023.

36) Fu, R.; Li, Z. Org. Lett. 2018, 20, 2342-2345.

37) Zhao, H.; Yang, K.; Zheng, H.; Ding, R.; Yin, F.; Wang, N.; Li, Y.; Cheng, B.; Wang, H.; Zhai, H. Org. Lett. 2015, 17, 5744-5747.

38) Kennedy, N.; Lu, G.; Liu, P.; Cohen, T. J. Org. Chem. 2015, 80, 8571-8582

39) Lai, J.; Chang, L.; Yuan, G. Org. Lett. 2016, 18, 3194-3197.

40) Ruzicka, R.; Barakova, L.; Klan, P. J. Phys Chem. 2005, 109, 9346-9353.

41) Smart, K. A., Mothes-Martin, E., Annaka, T., Grellier, M., \& Sabo-Etienne, S. Adv. Synth. Catal. 2014, 356, 759-764

42) Zhai, L.; Shukla, R.; Rathore, R. Org. Lett. 2009, 11, 3474-3477.

43) Alvarez-Bercedo, P., Martin, R. J. Am. Chem. Soc. 2010, 132, 17352-17353.

44) Abid, O.; Nawaz, M.; Ibad, F.N.; Khera, R.A.; laroshenko, V.; Langer, P. Org. Biomol. Chem. 2011, 2185-2191. 
45) Jolliffe, J. D.; Armstrong, R. J.; Smith, M. D. Nature Chem. 2017, 9, 558-562.

46) Barbero, N.; Martin, R. Org. Lett. 2012, 14, 796-799.

47) El-Deeb, I. Y.; Tian, M.; Funakoshi, T.; Matsubara, R.; Hayashi, M. Eur. J. Org. Chem. 2017, 2, 409413.

48) Anderson, E.D.; Boger, D.L. J. Am. Chem. Soc. 2011, 133, 12285-12292.

49) Bodnar, B.S.; Vogt, P. F. J. Org. Chem. 2009, 74, 2598-2600.

50) Fricke, C.; Dahiya, A.; Reid, W.B.; Schoenebeck, F. ACS Catal. 2019, 9, 9231-9236.

51) Neilsen, D.-T. C.; Burés, J. Chem. Sci. 2019, 10, 348-353.

52) Bernini, R.; Cacchi, S.; Fabrizi, G.; Forte, G.; Petrucci, F.; Prastaro, A.; Niembro, S.; Shafir, A.; Vallribera, A. Green Chem. 2010, 12, 150-158.

53) Dobele, M.; Vanderheiden, S.; Jung, N.; Brase, S. Angew. Chem. Int. Ed. 2010, 49, 5986-5988.

54) Tobisu, M,; Xu, T.; Shimasaki, T.; Chatani, N. J. Am. Chem. Soc. 2011, 133, 19505-19511.

55) Zhou, W-J.; Wang, K-H.; Wang, J-X.; Huang, D-F. Eur. J. Org. Chem. 2010, 3, 416-419.

56) Zhou, C-Y.; Chan, P. W. H.; Che, C-M. Org. Lett. 2006, 8, 325-328.

57) Kumar, R.; Sharma, A.; Sharma, N.; Kumar, V.; Sinha, A.K. Eur. J. Org. Chem. 2008, 33, 5577-5582. 